\title{
Textur- und Mikrostrukturanalysen an Materialien für den direkten und indirekten Zahnersatz
}

\author{
Dissertation \\ zur Erlangung des mathematisch-naturwissenschaftlichen Doktorgrades \\ „Doctor rerum naturalium" \\ der Georg-August-Universität Göttingen
}

im Promotionsprogramm Geowissenschaften

der Georg-August University School of Science (GAUSS)

vorgelegt von

Christiane Diana Hartmann

aus Höxter

Göttingen 2014 
$\underline{\text { Betreuungsausschuss: }}$

Priv.-Doz. Dr. Helmut Klein, Kristallographie, Georg-August-Universität

Dr. Heidrun Sowa, Kristallographie, Georg-August-Universität

Prof. Dr. Werner F. Kuhs, Kristallographie, Georg-August-Universität

$\underline{\text { Mitglieder der Prüfungskommission: }}$

Referent: Priv.-Doz. Dr. Helmut Klein, Kristallographie, Georg-August-Universität Korreferentin: Dr. Heidrun Sowa, Kristallographie, Georg-August-Universität

Korreferent: Prof. Dr. Werner F. Kuhs, Kristallographie, Georg-August-Universität

weitere Mitglieder der Prüfungskommission:

Prof. Dr. Sharon Webb, Mineralogie, Georg-August-Universität

Priv.-Doz. Dr. med. dent. Nikolaus Gersdorff, Prothetik, Universitätsmedizin Göttingen

Dr. Bernd Leiss, Strukturgeologie, Georg-August-Universität

Tag der mündlichen Prüfung: 13.02.2014 


\section{Danksagung}

Hiermit möchte ich besonderen Dank an alle ausrichten, die meine Doktorarbeit möglich gemacht und mich unterstützt haben:

Herrn PD Dr. Helmut Klein danke ich für die Möglichkeit, dass ich die Doktorarbeit bei ihm durchführen konnte, für die Betreuung meiner Arbeit und die fachlichen Diskussionen.

Herrn Prof. Dr. Werner F. Kuhs danke ich, dass ich in seiner Abteilung promovieren konnte und dass er das Korreferat übernommen hat.

Frau apl. Prof. Dr. Heidrun Sowa danke ich für die fachlichen Diskussionen, für die Unterstützung bei den Synchrotronmessungen am DESY und für die Übernahme des Korreferats.

Herrn Dr. Lars Raue danke ich für die Bereitstellung des Themas und dass ich mit ihm zusammen in demselben Projekt arbeiten konnte. Außerdem möchte ich mich für die Unterstützung bei den Durchführungen der Messungen und für die Hilfestellung bei technischen Problemen bedanken.

Prof. Dr. Nikolaus Gersdorff und Dr. Matthias Rödiger danke ich für die Bereitstellung der Proben und für die Erläuterungen zu zahntechnischen Fragestellungen.

Herrn Ulf Kahmann, Herrn Heiner Bartels und Klaus Haepe danke ich für die Hilfestellungen zur Präparation der Proben und für die Unterstützung bei technischen Problemen.

Imke Janssen, Nina Pukallus und Kathrin Nützmann danke ich für die Unterstützung bei den Synchrotronmessungen in Hamburg.

Bei Peter Zietlow und Kathrin Nützmann möchte ich mich dafür bedanken, dass sie ihr Kristallographie-Projekt in meinem Themenbereich gemacht haben und dadurch weitere Diskussionen angeregten.

Harald Tonn und Gabriele Mengel danke ich für die Herstellung der Dünn- und Dickschliffe für die Untersuchungen am REM und an der Mikrosonde.

Dr. Andreas Kronz danke ich für die Betreuung an der Mikrosonde.

Cornelia Mewes und dem Institut für Materialphysik Göttingen danke ich für die Benutzung und Einweisung in das Härteprüfgerät Fischerscope ${ }^{\circledR}$ H100SMC.

Dr. Horst Purwin danke ich für die Diskussionen zu Fragestellungen am REM und an der Mikrosonde.

Der Deutschen Forschungsgemeinschaft danke ich für die finanzielle Unterstützung.

Mein persönlicher Dank geht an meine Familie, besonders an meine Mutter Siglinde Hartmann und an meinen Freund Dr. Horst Purwin, die mich fortwährend seelisch unterstützt und ermutigt haben. Außerdem möchte ich mich beim esg-Chor „Spirit of Glory“ bedanken, bei dem ich mich nach der Arbeit entspannen konnte. 


\section{Abstract}

Physical properties like hardness, e-modulus and thermal expansion of dental materials are supposed to be nondirectional (isotropic), although these materials can possess directional (anisotropic) properties due to their mineralogical composition. Indeed, the ideal dental restorative should possess similar anisotropic physical properties like the restored tooth, which is - in the case of dental enamel - highly anisotropic (Raue \& Klein, 2011). Therefore, already existing materials are examined via x-ray analysis for their mineralogical composition and their anisotropic physical properties first and then compared with natural tooth.

Dental composites, denture resins and glass ionomer cements are composed of amorphous components, namely glass filler particles and resin, and show an isotropic physical behaviour: Vickers hardness and e-modulus are in the same range as for dentin, whereas their thermal expansion (35$\left.75 \cdot 10^{-6} / \mathrm{K}\right)$ is considerably higher than that of dentin and enamel $\left(11 \cdot 10^{-6} / \mathrm{K}\right.$ and $17 \cdot 10^{-6} / \mathrm{K}$; $\mathrm{Xu}$ et al. (1989)).

$\gamma_{2}$-free amalgam consists of the crystalline phases $\mathrm{Ag}_{2} \mathrm{Hg}_{3}, \mathrm{Ag}_{3} \mathrm{Sn}$ and $\mathrm{Cu}_{6} \mathrm{Sn}_{5}$. It shows no preferred orientation and no anisotropic behaviour. Its Vickers hardness and e-modulus (191 HV $0.03 / 20$ and $68 \mathrm{GPa}$, respectively) are between the values for dentin $(70 \mathrm{HV} 0.01 / 30$ and $2-$ $29 \mathrm{GPa}$; Schmitt et al., 2008; Kinney et al., 1999) and enamel (370 HV 0.01/30 and 10-169 GPa; Schmitt et al., 2008; Raue et al., 2011). Its thermal expansion $\left(25.5 \cdot 10^{-6} / \mathrm{K}\right.$ Kandil et al., 1989) is slightly higher than that of natural tooth.

Most dental ceramics are glass ceramics and show a complex mineral texture: the crystallites of the ceramics Cercon base and Vita In-Ceram Alumina are randomly oriented, whereas the ceramics Vita In-Ceram Zirkonia and Vitablocs Esthetic Line show a slightly preferred orientation (4mrd and 3mrd in ODF). Only the crystalline $\mathrm{Li}_{2} \mathrm{Si}_{2} \mathrm{O}_{5}$-phase of the glass ceramic IPS e.max Press shows a highly preferred orientation (11mrd in ODF). These crystal orientations result in slight anisotropies of the physical properties. With $\geq 650 \mathrm{HV} 0.01 / 20$ their Vickers hardness is markedly higher than that of natural tooth. With the exception of the high-perfomance ceramics, their e-moduli are between $60 \mathrm{GPa}$ and $98 \mathrm{GPa}$ and range between that of dentin and enamel. Their thermal expansion $\left(7.2-10.5 \cdot 10^{-6} / \mathrm{K}\right)$ is below that of dentin.

Gold alloys and non-precious alloys are coarse and contain precipitation phases which harden the alloys. Alloys for crowns have Vicker hardness numbers and e-modulus in the range of dental enamel, whereas the Co-Cr-Mo-alloy used for dental models is considerably harder and more elastic ( $582 \mathrm{HV} 0.03 / 20 ; E=211 \mathrm{GPa})$. Their thermal expansion is between that of dentin and enamel.

Titanium endodontic post and the dental implant Osseospeed show a highly preferred orientation (21mrd and $19 \mathrm{mrd}$ in ODF) and slightly anisotropic physical behaviour. Values for e-modulus and thermal expansion range between $159-167 \mathrm{GPa}$ and $7.62-9.29 \cdot 10^{-6} / \mathrm{K}$, respectively.

All in all the differences in the macroscopic physical properties of the examined dental materials in comparison to natural teeth are higher than the variations due to their crystalline composition and texture. Hence, new dental materials should be adapted more to human tooth in general and only in the next step their physical properties can be adapted to the anisotropic behaviour of dental enamel. The optimal dental restoration should be made from the same material as the natural tooth, thus hydroxylapatite. The hydroxylapatite should also have a similar microstructure as natural enamel, i.e. it should be just as fine grained as the hydroxylapatite in natural 
teeth and its crystallites should be grown epitaxial on enamel or dentine. First experiments are done whereupon more acid-resistant fluorapatite crystals instead of hydroxylapatite crystals were grown on dentine (Busch, 2008). However, it was not possible to grow the crystals in the same orientation as in dentine or enamel. But with the right crystal orientation of the fluorapatite crystals this would be the optimal dental restorative material, because it is very similar in its physical properties to natural tooth and it is more acid-resistant in comparison to hydroxylapatite. 


\section{Inhaltsverzeichnis}

\begin{tabular}{l} 
Danksagung \\
\hline
\end{tabular}

$\begin{array}{lr}\text { Abstract } & 4\end{array}$

1 Einleitung $\quad 9$

1.1 Motivation und Ziel der Arbeit . . . . . . . . . . . . . . . . . . . . . 9

1.2 Entwicklung von Zahnmaterialien ...................... 9

1.2.1 Gold in der Zahnmedizin . . . . . . . . . . . . . . . . . . . . . . . . 9 9

1.2.2 Amalgam in der Zahnmedizin . . . . . . . . . . . . . . . . . . . . 10

1.2.3 Keramik in der Zahnmedizin . . . . . . . . . . . . . . . . . . . 11

1.2.4 Kunststoffe in der Zahnmedizin . . . . . . . . . . . . . . . . . . . . . 13

1.3 Texturen von Zahnmaterialien . . . . . . . . . . . . . . . . . . . . . 14

2 Theoretische Grundlagen $\quad 15$

2.1 Elektronenmikroskopische Untersuchungen am Rasterelektronenmikroskop und an der Mikrosonde ... . . . . . . . . . . . . . . . . . . . . . 15

2.2 Quantitative Phasenanalyse . . . . . . . . . . . . . . . . . . . . 20

2.2.1 Bestimmung des Glasanteils mittels röntgenographischer Methoden . . . . 25

2.3 Textur und anisotrope Eigenschaften von polykristallinen Materialien . . . . . . . 26

2.3.1 Darstellung von Orientierungen . . . . . . . . . . . . . . . . . 27

2.3.2 Berechnung und Darstellung von Polfiguren . . . . . . . . . . . . . . . . . 29

2.3.3 Orientierungsverteilungen ...................... 31

2.3.4 Berechnung der Orientierungsverteilung mit Hilfe von symmetrischen verallgemeinerten Kugelfunktionen . . . . . . . . . . . . . . . . . . . . . . . . 31

2.3.5 Berechnung der Orientierungsverteilung nach der E-WIMV-Methode . . . 34

2.3.6 Fasertexturen .......................... 36

2.3.7 Berechnung von makroskopischen physikalischen Eigenschaften unter Berücksichtigung der Textur . . . . . . . . . . . . . . . . . . . . . 36

2.4 Ermittlung der Vickershärte und des E-Moduls mit dem instrumentellen Eindringverfahren ............................. 38

3 Material $\quad 40$

3.1 Direkte Füllmaterialien . . . . . . . . . . . . . . . . . . . . . . . . . . . . . . . 40

3.1.1 Amalgam ............................ 40

3.1.2 Dentalkomposite ........................ . . 41

3.1.3 Glasionomerzemente und Zn-Sulfat-Zemente . . . . . . . . . . . . . . . . 43

3.2 Indirekte Füllmaterialien und Werkstoffe für Zahnprothesen . . . . . . . . . . . . 45

3.2.1 Prothesenkunststoffe und Kunststoffzähne . . . . . . . . . . . . . . . . . . 45

3.2 .2 Dentalkeramiken ....................... 47

3.2.3 Metalllegierungen im Dentalbereich ..................... 52

3.3 Füllungen in extrahierten Zähnen . . . . . . . . . . . . . . . . . . . . . . 55

4 Durchführung der Experimente $\quad 56$

4.1 Herstellung der Proben . . . . . . . . . . . . . . . . . . . 56

4.2 Präparation der Proben . . . . . . . . . . . . . . . . . . . . . . . 57

4.3 Auflicht-Mikroskopie ......................... . 58 
4.4 Rasterelekronenmikroskop . . . . . . . . . . . . . . . . . . . . . . 59

4.5 Elektronenstrahl-Mikrosonde . . . . . . . . . . . . . . . . . . 59

4.6 Inhouse-Messungen am Bruker Smart Apex II . . . . . . . . . . . . . . . . . . . 59

4.7 Konventionelle Texturmessungen . . . . . . . . . . . . . . . . . . . 60

4.8 Messaufbau am Synchrotron . . . . . . . . . . . . . . . . . . . 6 . 61

4.9 Quantitative röntgenographische Phasen- und Texturanalyse . . . . . . . . . . 62

4.10 Berechnung der anisotropen physikalischen Eigenschaften eines polykristallinen Werkstoffs mit BEARTEX . . . . . . . . . . . . . . . . . . . 64

4.11 Anwendung des instrumentierten Eindringverfahrens zur Ermittlung der Vickershärte und des E-Moduls . . . . . . . . . . . . . . . . . . . 66

5 Ergebnisse $\quad 67$

5.1 Ergebnisse der Auflichtmikroskopie . . . . . . . . . . . . . . . . 67

5.2 Ergebnisse aus den Untersuchungen am REM . . . . . . . . . . . . . . . 69

5.3 Ergebnisse aus den Untersuchungen mit der Elektronenstrahlmikrosonde . . . . . 71

5.4 Ergebnisse der röntgenographischen Phasenanalyse . . . . . . . . . . . . . . 78

5.4.1 Phasenanalyse der direkten Füllmaterialien . . . . . . . . . . . . . . 78

5.4.1.1 Dentalamalgame Amalcap, regular und fast . . . . . . . . . 78

5.4.1.2 Dentalkomposite, Prothesenkunststoffe und Glasionomerzement . 78

5.4.1.3 Zinksulfatzemente . . . . . . . . . . . . . . . . 80

5.4 .2 Phasenanalyse der Keramiken . . . . . . . . . . . . . . . . . 81

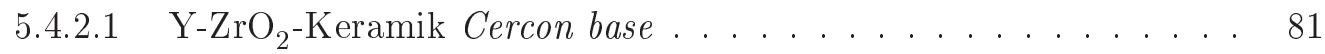

5.4.2.2 $\mathrm{Li}_{2} \mathrm{Si}_{2} \mathrm{O}_{5}$-Keramik IPS e.max Press . . . . . . . . . . . . . . 81

5.4.2.3 $\quad \mathrm{Li}_{2} \mathrm{Si}_{2} \mathrm{O}_{5}$-Keramik IPS e.max CAD . . . . . . . . . . . . 82

5.4.2.4 Feldspatkeramik Vitablocs Esthetic Line . . . . . . . . . . . . . . 82

5.4.2.5 Glasinfiltrationskeramik Vita In-Ceram Alumina . . . . . . . . . 83

5.4.2.6 Glasinfiltrationskeramik Vita In-Ceram Zirkonia . . . . . . . . . 83

5.4.3 Phasenanalyse der Dentalmetalle und Dentallegierungen . . . . . . . . . 84

5.4.3.1 Goldlegierung Degudent $U$. . . . . . . . . . . . . . . . 84

5.4.3.2 Goldlegierung Degulor $M \ldots$. . . . . . . . . . . 86

5.4.3.3 Titan-Wurzelstift der Firma Gebr. Brasseler . . . . . . . . . . 88

5.4.3.4 Titan-Implantat OsseoSpeed . . . . . . . . . . . . . . . 88

5.4.3.5 Ni-Cr-Mo-Legierung Wiron99 . . . . . . . . . . . . . . 89

5.4.3.6 Co-Cr-Mo-Legierung Remanium GM $800+\ldots . . . . . . . .991$

5.4 .4 Füllungen in extrahierten Zähnen . . . . . . . . . . . . . . . . . . . 93

5.4.4.1 Amalgamfüllung . . . . . . . . . . . . . . . . . . 93

5.4.4.2 Goldfüllung mit Unterfüllung . . . . . . . . . . . . . . . . . 93

5.5 Ergebnisse der röntgenographischen Berechnung von Glasgehalten in Glaskeramiken 96

5.6 Ergebnisse der Texturanalyse . . . . . . . . . . . . . . . . . . . . . 101

5.6.1 Dentalamalgame Amalcap regular und fast. . . . . . . . . . . . . . . 101

5.6.2 Dentalkomposite, Prothesenkunststoffe und Glasionomerzement . . . . . . 101

5.6 .3 Zinksulfatzemente . . . . . . . . . . . . . . . . . . . . . 102

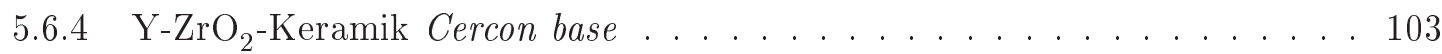

5.6.5 $\mathrm{Li}_{2} \mathrm{Si}_{2} \mathrm{O}_{5}-$ Keramik IPS e.max Press . . . . . . . . . . . . . . . . 103

$5.6 .6 \quad \mathrm{Li}_{2} \mathrm{Si}_{2} \mathrm{O}_{5}$-Keramik IPS e.max CAD . . . . . . . . . . . . . . . 107

5.6.7 Feldspatkeramik Vitablocs - Esthetic Line . . . . . . . . . . . . . . . . 107

5.6.8 Glasinfiltrationskeramik Vita In-Ceram Alumina . . . . . . . . . . . . . 111 
5.6.9 Glasinfiltrationskeramik Vita In-Ceram Zirkonia . . . . . . . . . . . . . 111

5.6.10 Goldlegierungen Degudent $U$ und Degulor $M$. . . . . . . . . . . . . . 112

5.6 .11 Titan-Wurzelstift . . . . . . . . . . . . . . . . . . . . . . . 113

5.6.12 Titan-Implantatsystem OsseoSpeed . . . . . . . . . . . . . . . . . 118

5.7 Berechnung der anisotropen physikalischen Eigenschaften aus Texturanalysen . . 120

5.7.1 Amalgame Amalcap regular und fast . . . . . . . . . . . . . . . . . . 120

5.7.2 Dentalkomposite, Prothesenkunststoffe und Glasionomerzement . . . . . . 120

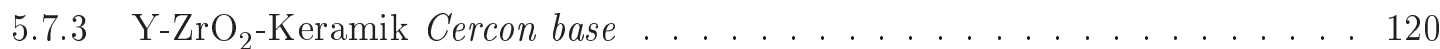

$5.7 .4 \quad \mathrm{Li}_{2} \mathrm{Si}_{2} \mathrm{O}_{5}-$ Keramik IPS e.max Press . . . . . . . . . . . . . . . . . 120

5.7.5 $\mathrm{Li}_{2} \mathrm{Si}_{2} \mathrm{O}_{5}$-Keramik IPS e.max CAD . . . . . . . . . . . . . . . 121

5.7.6 Feldspatkeramik Vitablocs - Esthetic Line . . . . . . . . . . . . . . . . . . 122

5.7.7 Glasinfiltrationskeramik Vita In-Ceram Alumina . . . . . . . . . . . . . . 123

5.7.8 Glasinfiltrationskeramik Vita In-Ceram Zirkonia . . . . . . . . . . . . . . 123

5.7.9 Goldlegierungen Degudent $U$ und Degulor $M$. . . . . . . . . . . . . 125

5.7 .10 Titan-Wurzelstift . . . . . . . . . . . . . . . . . . . . . . 125

5.7 .11 Titan-Implantat OsseoSpeed . . . . . . . . . . . . . . . . . 126

5.8 Ergebnisse aus dem instrumentierten Eindringverfahren $\ldots \ldots \ldots \ldots$

6 Fehlerbetrachtung 132

6.1 Fehlerquellen bei REM-Untersuchungen . . . . . . . . . . . . . . . . . . . . 132

6.2 Fehlerquellen bei Untersuchungen an der Elektronenstrahlmikrosonde . . . . . . . 132

6.3 Fehlerquellen bei der röntgenographischen Phasenanalyse . . . . . . . . . . . . . . 133

6.4 Fehlerquellen bei den Texturanalysen und der Berechnung der physikalischen Eigenschaften . . . . . . . . . . . . . . . . . . . . 133

6.5 Fehlerquellen beim instrumentierten Eindringverfahren zur Bestimmung der Vickershärte und des E-Moduls . . . . . . . . . . . . . . . . . . . 134

7 Diskussion

7.1 Anwendbarkeit der röntgenographischen Glaskonzentrationsbestimmung und Vergleich der Ergebnisse mit Herstellerangaben . . . . . . . . . . . . . . . . . 135

7.2 Vergleich der Texturberechnung nach der harmonischen Methode mit der Texturberechnung nach der E-WIMV-Methode . . . . . . . . . . . . . . . 138

7.3 Vergleich der Härte- und E-Modulwerte aus dem instrumentierten Eindringverfahren mit anderen Untersuchungen . . . . . . . . . . . . . . . . . . . . . 139

7.4 Vergleich der Ergebnisse aus Texturanalysen mit Ergebnissen aus dem instrumentierten Eindringverfahren und mit Literaturwerten . . . . . . . . . . . . . . 143

7.5 Vergleich der Eigenschaften der untersuchten Zahnmaterialien mit natürlichem Zahn146

$\begin{array}{ll}\text { A Tabellen } & 168\end{array}$

$\begin{array}{ll}\text { B Abbildungen } & 175\end{array}$ 


\section{Einleitung}

\subsection{Motivation und Ziel der Arbeit}

Zähne müssen im Leben eines Menschen sehr viel Arbeit beim Zerkleinern von Nahrung leisten, wobei sie besonders in unserem modernen Zeitalter stark von säure- und zuckerhaltigen Lebensmitteln angegriffen werden. Die Folge sind Abnahme des natürlichen Zahnschmelzes und Kariesbefall bis hin zur Entzündung des Zahnnerves und der Zahnwurzel. Um dennoch möglichst lange die Funktion der Zähne aufrecht zu erhalten, wird zum einen nach Mitteln zur Bekämpfung von Karies und zum anderen nach geeigneten Füllmaterialien und nach Materialien für den kompletten Zahnersatz geforscht. Im Idealfall sollten die Ersatzmaterialien gleiche physikalische Eigenschaften wie der natürliche Zahn besitzen. Dazu gehören u.a. Härte, Elastizität, thermische Ausdehnung, Farbe und Transluszenz. Außerdem ist eine gewisse Biegefestigkeit und Risszähigkeit nötig, um eine lange Haltbarkeit der Zahnrestauration zu erzielen.

Im Rahmen dieser Arbeit werden heute auf dem Markt zugängliche Zahnmaterialien für verschiedene Anwendungen auf ihre physikalischen Eigenschaften untersucht und mit den physikalischen Eigenschaften von natürlichen Zähnen verglichen.

\subsection{Entwicklung von Zahnmaterialien}

\subsubsection{Gold in der Zahnmedizin}

In der Zahnmedizin ist Gold das mit Abstand älteste verwendete Material. Es gibt sichere Belege, dass die Etrusker im 7. Jh. v. Chr. Ersatzzähne mit Golddraht bzw. Goldbändern befestigten, wobei als Ersatzzahn entweder der eigene Zahn (ohne Wurzel), tierische Zähne oder Elfenbein, vorzugsweise vom Nilpferd, verwendet wurden (Donaldson, 1980a). Weiterhin gibt es ein Manuskript des Arabers Albucasis aus dem späten 11. Jh. n. Chr., das Gold zur Befestigung von lockeren Schneidezähnen empfiehlt, wobei er Gold den Silberlegierungen und anderen Legierungen aufgrund seiner chemischen Beständigkeit gegen Speichel vorzieht (Donaldson, 1980a). Diese Techniken sind im Mittelalter in Vergessenheit geraten. Erst im 16. Jh. gibt es vermehrt medizinische Berichte bzw. Bücher, in denen auch Zahnbehandlungen erwähnt werden (Donaldson, 1980a; Prinz, 1923). Erste Hinweise auf das Auskratzen von Karies und Anwendung von Goldfüllungen kann man in dem 1530 in Leipzig veröffentlichten Buch „Artzney Büchlein“ von Michael Blum finden (Donaldson, 1980a). Allerdings waren der Aufbau und die Struktur von Zähnen sowie die Art von Zahnfäule zu der Zeit noch nicht verstanden, sodass der Nutzen der Goldfüllungen durch unzureichende Mundhygiene nur sehr gering war. Einen großen Fortschritt in der Zahnmedizin erreichte Fauchard im 18. Jh. (Fauchard, 1728), der erstmals detaillierte Beschreibungen vom Aufbau der Zähne und der Behandlung von Zahnfäule sowie von weiteren kieferchirurgischen Methoden in seinem Buch „Le Chirurgien Dentiste“ aufschrieb (Prinz, 1923). Dieses Buch gilt als erstes wissenschaftliches Buch der Zahnmedizin, das von anderen Medizinern vor seiner Veröffentlichung gründlich begutachtet wurde (Spielmann, 2007). Im 19. Jh. wurden schließlich Goldfüllungen nach der Goldfolie-Hämmertechnik häufiger angewendet, da es 
zu diesem Zeitpunkt bessere mechanische Präparationsgeräte gab (Donaldson, 1980a). Hierbei ist besonders eine Arbeit von Robert Arthur erwähnenswert, der die Methode des Goldfolienhämmerns ausführlich erläutert, wobei er auf die Nutzung von sterilen, weichgeglühten und kohäsiven Goldfolien hinweist (Arthur, 1857). Da der Prozess des Goldfolienhämmerns sowohl für den Zahnarzt als auch für den Patienten sehr langwierig und anstrengend ist, suchte man nach alternativen Methoden und Zahnersatzmaterialien. Es entstand die Idee von Gold-Inlays, die der Zahnarzt ohne ständige Anwesenheit des Patienten erstellen und ihm relativ zügig einsetzen kann. Im Zuge dessen wurde das Wachsausschmelzverfahren für Gold-Inlays Anfang des 20. Jhs. entwickelt (Taggart, 1907). Neben der Anwendung von Gold oder Goldlegierungen für Zahnfüllungen, Inlays, Onlays und Kronen wurden sie seit der Mitte des 17. Jhs. auch als Basis für Gebisse und ab dem 19. Jh. für Stiftkronen verwendet (Donaldson, 1980b). Außerdem wurde Gold schon früh für kieferorthopädische Zwecke (z.B. Korrektur von Zahnfehlstellungen) eingesetzt (Fauchard, 1728).

Bis heute wird weiter an der Verbesserung von Goldlegierungen in Hinsicht auf ihre Verarbeitbarkeit und ihre physikalischen Eigenschaften wie Festigkeit und thermische Ausdehnung gearbeitet. Je nachdem welche Metalle dem reinen Gold hinzu legiert werden, ändern sich die physikalischen Eigenschaften der Goldlegierungen. Dabei ist besonders der Prozess der Ausscheidungshärtung wichtig, wobei durch das Ausscheiden einer in der Goldlegierung fein verteilten zweiten (oder dritten) Phase die Festigkeit und Härte der Goldlegierung wesentlich erhöht wird. Durch das gezielte Hinzulegieren anderer Metalle wurde der Einsatzbereich von Gold in der Zahnmedizin erheblich ausgeweitet. Mittlerweile werden Goldlegierungen für Inlays, Onlays, (Teil-)Kronen, Wurzelstifte, Gebissgerüste, direkte Füllungen und Spangendrähte eingesetzt (Knosp et al., 1981). Für die verschiedenen Einsatzgebiete gibt es dementsprechend verschiedene Goldlegierung-Standards (Knosp et al., 2003). Kronen, Inlays und Onlays werden zudem gerne mit Kunststoff oder Keramik verblendet, um eine bessere Ästhetik zu erzielen. Die Methode des Goldfolienhämmerns wird aufgrund der langen Prozedur und der auffälligen Farbe heutzutage kaum eingesetzt, wobei aktuelle wissenschaftliche Untersuchungen gezeigt haben, dass ihre Anwendung für kleinere Füllungen aufgrund ihrer langen Haltbarkeit immer noch gerechtfertigt ist (Kamann, 2000).

\subsubsection{Amalgam in der Zahnmedizin}

Auf der Suche nach kostengünstigen Alternativen zu Goldfüllungen ist man schließlich zu Amalgamfüllungen übergegangen, mit denen Kavitäten durch Stopfen wesentlich leichter als nach der Goldhämmermethode gefüllt werden können. Zum ersten großräumigen Einsatz von Amalgamen in Europa und in den USA kam es zu Beginn des 19. Jhs. Es bildete damals nicht nur eine sehr günstige Alternative zu den bisher üblichen Goldfüllungen, sondern konnte aufgrund seiner guten Verarbeitbarkeit oft auch dann eingesetzt werden, wenn die zu füllende Kavität nicht mit einer Goldfüllung restauriert werden konnte (Hyson, 2006). Allerdings ist schon fast von Anfang an umstritten, ob das in den Amalgamen enthaltene Quecksilber aus dem Amalgam entweicht und wie groß die tatsächliche Gefahr für die Gesundheit von Amalgamträgern ist (Hyson, 2006). Die immer wieder neu gestellte Gesundheitsfrage führte zur Verbesserung der Dentalalmalgame und auch zu einem sorgfältigeren Umgang mit ihnen. Zu Beginn wurden Kupferamalgame eingesetzt, die schon im 19. Jh. durch Silberamalgame verdrängt wurden. Die Bildung der Silberamalgame 
beruht auf einer chemischen Reaktion, bei der die $\gamma-\mathrm{Ag}_{3} \mathrm{Sn}$-Phase mit flüssigem Quecksilber zu den festen Verbindungen $\gamma_{1}-\mathrm{Ag}_{2} \mathrm{Hg}_{3}$ und $\gamma_{2}-\mathrm{Sn}_{x} \mathrm{Hg}(\mathrm{x}=7$ bis 8$)$ reagiert:

$$
\mathrm{Ag}_{3} \mathrm{Sn}_{(s)}+\mathrm{Hg}_{(l)} \Rightarrow \mathrm{Ag}_{2} \mathrm{Hg}_{3(s)}+\mathrm{Sn}_{x} \mathrm{Hg}_{(\mathrm{s})}+\mathrm{Ag}_{3} \mathrm{Sn}_{(\mathrm{s})}
$$

mit $_{(s)}$ und ${ }_{(l)}$ als Bezeichnung für die Aggregatzustände fest (s) und flüssig (l).

Da sich die $\gamma_{2}$-Phase als nicht korrosionsbeständig erwies, wurde im Laufe des 20. Jahrhunderts den Silberamalgamen soviel Kupfer hinzugefügt, dass sich statt der korrosionsunbeständigen $\gamma_{2}$-Phase die $\eta$ - $\mathrm{Cu}_{6} \mathrm{Sn}_{5}$-Phase bildet:

$$
\mathrm{Ag}_{3} \mathrm{Sn}_{(s)}+\mathrm{Ag}-\mathrm{Cu}_{(s)}+\mathrm{Hg}(l) \Rightarrow \mathrm{Ag}_{2} \mathrm{Hg}_{3(s)}+\mathrm{Cu}_{6} \mathrm{Sn}_{5(s)}+A g 3 S n_{(s)}+\mathrm{Ag}-\mathrm{Cu}_{(\mathrm{s})}
$$

$\operatorname{mit}_{(s)}$ und $(l)$ als Bezeichnung für die Aggregatzustände fest (s) und flüssig (l).

Um die Quecksilberbelastung von Zahnärzten und Patienten weiterhin zu senken, werden heutzutage nur noch $\gamma_{2}$-freie Silberamalgame verwendet, die zudem in sicher verschlossenen Applikationskapseln vom Hersteller geliefert werden. In einer Hälfte befindet sich das Pulver aus Silber, Zinn und Kupfer und in der anderen Hälfte das flüssige Quecksilber. Die beiden Phasen sind durch eine Membran getrennt. Direkt vor Gebrauch wird die Kapsel in einen Schüttler eingebaut, in dem die Membran zerstört wird und Pulver und Flüssigkeit ca.10-15 sec. gemischt werden. Das fertige Amalgam ist nun gebrauchsfertig und muss zügig verarbeitet werden, bevor es durch die chemische Reaktionen ausgehärtet wird.

Trotz der Weiterentwicklung der Dentalamalgame, womit eine starke Abnahme der Quecksilberabgabe einhergeht, ihrer guten Verarbeitbarkeit und Haltbarkeit werden heute relativ wenige Amalgamfüllungen in den Industrieländern neu gelegt, da sie ästhetisch nicht ansprechend sind und die tatsächlichen gesundheitlichen Auswirkungen sehr umstritten sind (Hanson \& Pleva, 1991; Hyson, 2006; Uçar \& Brantley, 2011).

\subsubsection{Keramik in der Zahnmedizin}

Vor der Entwicklung der Porzellanherstellung in Europa (um 1720) wurden entweder menschliche oder tierische Zähne, Elfenbein (vorzugsweise vom Nilpferd) oder Rinderknochen als ästhetische Zahnersatzmaterialien verwendet (Kelly et al., 1996; Donaldson, 1980a). Die Idee eines emaillierten Metallgebisses wird erstmals von Fauchard (1728) beschrieben. Das erste erfolgreiche Porzellangebiss wurde schließlich 1774 von dem Apotheker Alexis Duchateau mit Hilfe des Zahnarztes Nicholas Dubois de Chemant hergestellt (Kelly et al., 1996). Im 19. Jh. entwickelte Elias Wildman höher transluszentes Porzellan für Inlays (Southan, 1975). Außerdem führte Giuseppe Fonzi Platin-Stiftkronen ein, wobei diese erst durch den verbesserten Verbund von Platin und Porzellan Ende des 19. Jhs. vermehrt eingesetzt wurden (Kelly et al., 1996; Al-Wahadni, 1999). Die ersten Porzellan-Jacketkronen wurden 1889 von Charles H. Land eingeführt (Al-Wahadni, 1999). Im 20. Jh. wurden bei Metall-Keramik-Restaurationen neben Platinlegierungen auch Goldlegierungen eingesetzt (Brecker, 1956) und das Aufbrennen der Keramikverblendung im Vakuum eingeführt (Al-Wahadni, 1999). Keramikverblendete Goldkronen werden heute noch standardmäßig eingesetzt. Das verwendete „Dentalporzellan“ (15-25 Gew\% $\mathrm{SiO}_{2}, 60$ - 80 Gew.\% Feldspat, 0 5 Gew.\% Kaolin) unterscheidet sich allerdings in der chemischen Zusammensetzung deutlich von 
anderen Gebrauchsporzellanen (20 - $30 \mathrm{Gew} \% \mathrm{SiO}_{2}, 12$ - 30 Gew.\% Feldspat, 40 - 70 Gew.\% Kaolin) und sollte daher nicht als Porzellan, sondern als Dentalkeramik bezeichnet werden (Eichner \& Kappert, 1996). Außerdem wird der Keramikverblendung Leucit zur Erhöhung des thermischen Ausdehnungskoeffizienten hinzufügt, um die durch das Aufbrennen der Keramik auf das Metallgerüst entstehenden Restspannungen möglichst gering zu halten (Kelly et al., 1996). Da die Ästhetik dieser keramikverblendeten Kronen und Brücken mit Metallgerüst nicht für alle Indikationen als ausreichend empfunden wurde, wurde weiter nach vollkeramischen Systemen geforscht. In den 80ern kam schließlich von der Firma Detrey Dentsply das erste Vollkeramik-System für Kronen auf den Markt (Kelly et al., 1996): Dicor, eine glimmerhaltige Guss-Glaskeramik, die aufgrund ihrer geringen Festigkeit heute jedoch nicht mehr verwendet wird. Kurz darauf wurden weitere Keramiksysteme eingeführt, die auf Glas, teilkristallinem oder ganzkristallinem Material und auf unterschiedlichen Verarbeitungstechniken basieren. In der Grundidee sind diese Systeme bis heute erhalten. Von der Verarbeitungstechnik her wird zwischen Gusskeramik, heiß gepresster Keramik und Keramiken nach dem Schlickerverfahren oder nach der CAD/CAM-Methode $(\mathrm{CAD} / \mathrm{CAM}=$ Computer-aided design/computer-aided manufacturing) bzw. Kopierschleifverfahren unterschieden:

- Gussverfahren: Ausgangsmaterial aus Glas und evtl. kristallinen Bestandteilen wird soweit erhitzt, dass man es in eine Modellform gießen kann.

- (Heiß-)Pressverfahren: teilkristallines Ausgangsmaterial wird soweit erwärmt, dass es sich in eine Modellform pressen lässt.

- Schlickerverfahren: Suspension aus Keramikpulver und Wasser wird mit einem Pinsel aufgetragen und dann gesintert (Beispiel: Vita In-Ceram $\AA$ ).

- Kopierschleifverfahren (CAM-Verfahren): ein fertiges (Kunststoff-)Modell wird digital erfasst und auf einen Fräskörper übertragen, der vollkommen kristallin oder teilkristallin sein kann (Beispiel: Cerec @).

- CAD/CAM-Verfahren: nach der digitalen Erfassung des Zahnmodells wird die Restauration am Computer simuliert und anschließend über eine Fräsmaschine an einem Keramik-

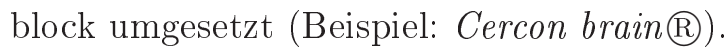

Gerade durch die Einführung des CAD/CAM-Verfahrens wurde der Bereich der möglichen Materialien für den dentalkeramischen Zahnersatz deutlich erweitert. Z.B. sind ZirkoniumdioxidKeramiken im fertigen Zustand nur unter hohem Geräteverschleiß verarbeitbar, wohingegen das $\mathrm{CAD} / \mathrm{CAM}$-System die Bearbeitung von nur vorgesinterten und somit weicheren $\mathrm{ZrO}_{2}$-Blöcken erlaubt, da die Schrumpfung der Keramik während des Sinterungsprozesses präzise berechnet werden kann. 
Anerkannte Vollkeramik-Systeme basieren heute auf den unterschiedlichsten Ausgangsmaterialien. Die am häufigsten verwendeten Minerale sind:

- Feldspat

- Korund

- Lithiumdisilikat

- Zirkoniumdioxid

- Spinell

Des Weiteren werden den Keramiken Flussmittelzusätze (Bsp.: $\mathrm{K}_{2} \mathrm{CO}_{3}, \mathrm{Na}_{2} \mathrm{CO}_{3}, \mathrm{~K}_{3} \mathrm{PO}_{4}$ oder Borax) bzw. typische Netzwerkwandler wie (Erd-)Alkalimetalloxide für Glaskeramiken auf Silikatbasis zur Senkung der Verarbeitungstemperatur, Opaker (z.B. $\mathrm{ZnO}, \mathrm{ZrO}_{2}$ und $\mathrm{TiO}_{2}$ ) und verschiedene Pigmente (z.B. Fe-, Cr-, Ir-, Ti- oder Mn-Oxide) hinzugefügt (Eichner \& Kappert, 1996).

\subsubsection{Kunststoffe in der Zahnmedizin}

Die ersten Kunststoffe wurden vor ca. 150 Jahren in der Zahnmedizin eingesetzt: Guttapercha, das heute noch für endodentische Wurzelkanalfüllungen verwendet wird, sowie Vulkanit und Zelluloid (Rueggeberg, 2002). Ein wesentlicher Vorteil dieser frühen Kunststoffe war, dass sie im Vergleich zu den bisher verwendeten Materialien wie Elfenbein und Gold (im Fall von Zahnprothesen) kostengünstige Alternativen darstellten. Allerdings hatte Vulkanit eine unattraktive dunkelbraune Farbe und Zelluoid eine Tendenz sich mit der Zeit grünlich zu verfärben (Rueggeberg, 2002). Anfang des 20. Jahrhunderts wurden weitere Kunststofftypen entwickelt und in den 60er Jahren erfand Bowen einen Kunststoff auf Bis-GMA-Basis (BisphenylA-Glycidyl-Methacrylat), dem er Füllkörper zur Verbesserung der physikalischen Eigenschaften hinzugefügt hatte (Bowen, 1963, 1965). Im Weiteren wurden die Eigenschaften durch Hinzufügen von Inhibitor- und Initiatorsystemen, Katalysatoren sowie verschiedene Füllpartikel verbessert. Ganz allgemein können daher heutige Dentalkomposite als ein Verbundwerkstoff aus organischem Polymerisat und anorganischen Füllkörpern betrachtet werden. Für das Polymerisat können verschiedene Monomer- und Co-Monomersysteme verwendet werden, deren häufigste Vertreter im Folgenden genannt sind (Graf, 2013):

- Bis-GMA (Bisphenyl-A-Glycidyl-Methacrylat),

- TEGDMA (Triethylenglycoldimethacrylat),

- DEGMA (Diethylenglycoldimethacrylat)

- und HEMA (Hydroxy-ethyl-methacrylat). 
Die Füllkörper bestehen zu einem großen Teil aus amorphem $\mathrm{SiO}_{2}$ oder Al-Si-Gläsern, die mit $\mathrm{La}, \mathrm{Zn}, \mathrm{Zr}$, Sr und/oder $\mathrm{Yb}$ angereichert sind. Teilweise wird auch $\mathrm{YbF}_{3}$ als Füllpartikel eingesetzt. Da die Füllkörper im Gegensatz zur Polymermatrix hydrophil sind, werden sie für einen besseren chemischen Verbund silanisiert. Neben der Art der Füllkörper ist auch ihre Korngrößenverteilung (Makro-, Mikro- und Nanofüllkörper) wichtig, weil die physikalischen Eigenschaften wie Viskosität, Biegefestigkeit, Abrasionsfestigkeit, Polymerisationsschrumpfung und thermische Ausdehnung des Endproduktes durch kontrolliertes Abstimmen der Korngrößenverteilung und des Füllkörpergehalts eingestellt werden können.

\subsection{Texturen von Zahnmaterialien}

Bisher wurden Texturen von Zahnmaterialien nicht quantitativ untersucht. Jedoch rückte in den letzten 10 Jahren der Einfluss der Kristallorientierungen auf die physikalischen Eigenschaften von Vollkeramiksystemen in das Interesse wissenschaftlicher Studien, wobei insbesondere die Vollkeramiksysteme Empress I (Leucit-verstärkte Glas-Keramik) und Empress II $\left(\mathrm{Li}_{2} \mathrm{Si}_{2} \mathrm{O}_{5}\right.$ verstärkte Glaskeramik) untersucht wurden (Albakry et al., 2003, 2004; Guazzato et al., 2004). Für die Gruppe der $\mathrm{Li}_{2} \mathrm{Si}_{2} \mathrm{O}_{5}$-Glaskeramiken zeigten Thiel \& Schnapp (1998) an einer heiß gepressten Keramik mit definierter chemischer Zusammensetzung (Bestandteile: $\mathrm{SiO}_{2}, \mathrm{Li}_{2} \mathrm{O}$, $\mathrm{B}_{2} \mathrm{O}_{3}, \mathrm{~K}_{2} \mathrm{O}, \mathrm{Al}_{2} \mathrm{O}_{3}$ und $\mathrm{P}_{2} \mathrm{O}_{5}$ ), dass sich die pseudo-orthorhombischen $\mathrm{Li}_{2} \mathrm{Si}_{2} \mathrm{O}_{5}$-Kristalle beim Strangpressverfahren bezüglich ihrer c-Achse orientiert anordneten und wie sich diese bevorzugte Kristallorientierung auf Vickershärte und Bruchzähigkeit ihrer $\mathrm{Li}_{2} \mathrm{Si}_{2} \mathrm{O}_{5}$-Glaskeramik auswirkte. Ähnliche Studien liegen zu Glaskeramiken auf Glimmer-Basis vor (Denry et al., 2002). In der Studie von Guazzato et al. (2004) wurden außerdem die Gerüstkeramiken Vita In-Ceram Alumina und Zirkonia nach der "Slip casting"-Technik (Schlickergießen) sowie als Glas-infiltrierter Block (CAD/CAM-Verfahren) untersucht. Für die Keramik Vita In-Ceram Alumina (,Slip casting") wurde anhand von Sekundärelektronen-Bildern eine bevorzugte Ausrichtung elongierter Korundkörner beobachtet (Guazzato et al., 2004). Trotzdem wurden alle physikalischen Eigenschaften wie Bruchzähigkeit, E-Modul und Härte nur richtungsunabhängig untersucht.

Daher soll im Rahmen der vorliegenden Doktorarbeit genauer auf die Texturen verschiedener Dentalwerkstoffe und auf die aus der Textur resultierenden physikalischen Eigenschaften eingegangen werden. 


\section{Theoretische Grundlagen}

\subsection{Elektronenmikroskopische Untersuchungen am Rasterelektronenmikroskop und an der Mikrosonde}

Sowohl mit der Elektronenstrahlmikrosonde (EMS) als auch mit dem Rasterelektronenmikroskop (REM) wird die Probe mit Hilfe eines fokussierten Elektronenstrahls analysiert. Trotz großer Überschneidungen dieser Methoden, kann es sinnvoll sein, beide parallel anzuwenden, da sie auf unterschiedliche Analyseverfahren spezialisiert sind. Die EMS wird hauptsächlich dafür eingesetzt, die chemische Zusammensetzung einer Probe mit hoher Ortsauflösung (5-10 $\mu \mathrm{m})$ zu erhalten, während sich das REM besser für orts- und tiefenauflösende Sekundärelektronen-Bilder zur Darstellung von Probenoberflächen eignet. Zusammen können beide Methoden zur Charakterisierung einer Probe, d.h. zur Oberflächenbeschreibung sowie Mineralbestimmung herangezogen werden.

\section{Wechselwirkung der Elektronen mit Materie}

Die Wechselwirkung der beschleunigten Elektronen (ca. 5-30 keV) mit der Probe lässt sich grob in zwei Arten der Wechselwirkung, der elastischen und der unelastischen Streuung der Elektronen, unterteilen. Bei der elastischen Streuung werden die Elektronen durch Wechselwirkung mit dem positiv geladenem Atomkern abgelenkt, wobei die kinetische Energie der Elektronen fast vollständig erhalten bleibt. Wenn die Ablenkung der Elektronen mehr als $90^{\circ}$ entspricht, sodass die Elektronen aus der Probe gelenkt werden, spricht man von Rückstreuelektronen (RE, Abb. 1). Im Fall der unelastischen Streuung der Elektronen treten mehrere physikalische Prozesse auf (Abb. 1):

- Sekundär-Elektronen (oberflächennah, geringe Energie)

- Röntgenstrahlen (größere Emissionstiefe, höhere Energie)

- Auger-Elektronen (oberflächennah, geringe Energie)

- Kathodolumineszenz (oberflächennah, geringe Energie)

Da für die Analyse der Dentalmaterialien neben den Rückstreuelektronen (RE) nur die SekundärElektronen (SE) und charakteristische Röntgenstrahlen wichtig sind, wird im folgenden Abschnitt kurz auf diese Effekte eingegangen.

Sekundär-Elektronen sind aus den den obersten Nanometern der Probe emittierte Elektronen, die durch den Primärelektronenstrahl angeregt wurden und eine wesentlich geringere Energie als die RE haben.

Röntgenstrahlen werden durch den einfallenden Elektronenstrahl auf zwei verschiedene Arten produziert. Einerseits werden die Elektronen durch das elektrische Feld eines Atomkerns abgelenkt, wobei Energie in Form von Röntgenstrahlung frei wird. Je nachdem wie sehr ein 


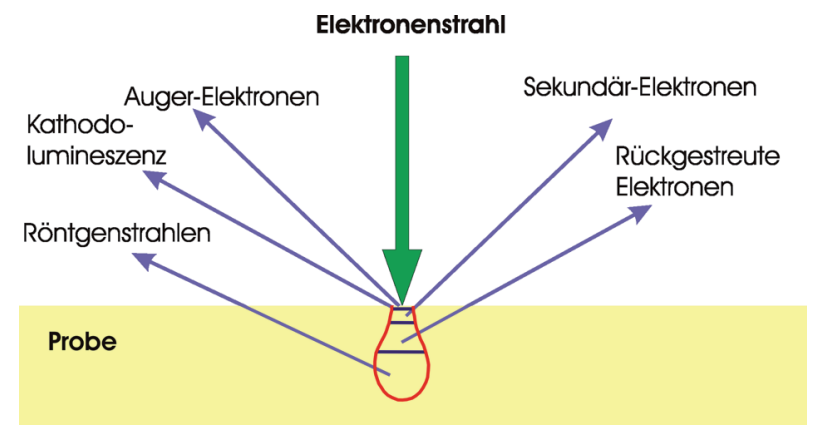

Abb. 1: Auftretende Wechselwirkungen des Elektronenstrahls mit der Probe.

Elektron eine Ablenkung erfährt, wird unterschiedlich viel Energie als Röntgenphoton freigesetzt, sodass die Röntgenstrahlung aller abgelenkten Elektronen ein kontinuierliches Energiespektrum (Bremsspektrum) aufweist, wobei die maximale Energie der Energie des einfallenden Elektronenstrahls entspricht. Andererseits werden kernnahe Elektronen durch die einfallenden Elektronen aus ihrer „Elektronenschale“ (z.B. K-Schale) geschlagen und die entstandene Lücke von Elektronen aus Elektronenschalen größerer Ordnung (z.B. L-Schale) aufgefüllt. Bei diesem Prozess wird je nach Element und je nach Orbital eine bestimmte Menge an Energie frei, die als charakteristische Röntgenstrahlung an die Umgebung abgegeben wird. Die Energie dieser Strahlung nimmt nach dem Moseleyschen Gesetz $\frac{1}{\lambda} \approx a(Z-b)^{2}$ (Wellenlänge $\lambda \approx \frac{1}{E}, a \neq b=$ Konstanten) mit steigender Ordnungszahl $Z$ zu (Moseley, 1913). Da nur die charakteristische Röntgenstrahlung elementspezifisch ist, wird auch nur diese für die Probenuntersuchung mit dem REM und der EMS herangezogen.

\section{Aufbau von REM und EMS}

Der Aufbau von REM und EMS ist vom Prinzip her sehr ähnlich und unterscheidet sich nur in relativ wenigen, aber dafür entscheidenden Komponenten. Folgende Komponenten werden sowohl für das REM als auch für die EMS benötigt:

- leistungsfähige Kathode (z.B. aus W oder $\mathrm{LaB}_{6}$ ) zur Erzeugung des Elektronenstrahls,

- Vakuum-System (Minimum: $10^{-5}$ mbar in der Probenkammmer),

- Elektronenlinsen-System zur Fokussierung des Elektronenstrahls,

- Blende zur Einstellung der Elektronenstrahlgröße,

- Probenhalter,

- SE-, RE-, Röntgen-Detektoren sowie weitere Detektoren nach Bedarf.

Sekundär- und Rückstreuelektronen können mit einem Everhart-Thornley-Detektor detektiert werden, der sowohl im REM als auch in der EMS verwendet wird. Dieser Detektor ist ein 
Szintillations-Detektor mit einem vorgeschalteten Gitter, das bei positiver Ladung SE und bei negativer Ladung RE zum Detektor durchlässt. Allerdings ist die Effizienz für RE relativ gering, sodass im REM für gute RE-Bilder mit einem scharfen Massekontrast ein Solid-State-Detektor benutzt wird, der koaxial zum Elektronenstrahl direkt oberhalb der Probe angebracht wird.

Die Detektion der Röntgenstrahlung zur Bestimmung der vorhandenen Elemente und ihrer Konzentrationen unterscheiden sich bei REM und EMS dagegen wesentlich. Im REM wird die charakteristische Röntgenstrahlung verschiedener Elemente simultan mit einem energiedispersiven Solid-State-Detektor (meist Li-Dotierter Si-Halbleiter ,,Si $(\mathrm{Li}) “$, seltener sehr reiner Ge-Halbleiter „HPGe") gemessen, während in der EMS ein wellenlängendispersives System verwendet wird. Beim wellenlängendispersiven System werden die Intensitäten für festgelegte Wellenlängen nacheinander mit Hilfe von Monochromatorkristallen in verschiedenen Winkelstellungen erfasst.

\section{Analyse mit einem energiedispersiven Detektor}

Die eintreffenden Röntgenstrahlen lösen im Detektor Elektronen-Loch-Paare und dadurch einen Stromfluss aus, der nach Vorverstärkung mittels eines Vielkanalanalysators in ein Energiespektrum konvertiert wird. Da die Anzahl der entstehenden Elektronen-Loch-Paare, die durch Röntgenphotonen gleicher Energie ausgelöst werden, statistisch variiert, erhält man im Energiespektrum keine scharfen, sondern breite Peaks in der Form einer Gaussverteilung.

Das gemessene Energiespektrum setzt sich aus dem Untergrundspektrum der Bremsstrahlung und den Peaks der charakteristischen Röntgenstrahlen zusammen (Vgl. Kap. 2.2). Daneben können auch folgende Artefakte auftreten:

- $\mathrm{Si}(\mathrm{Li})$-Halbleiter wird selbst zu charakteristischer Röntgenstrahlung angeregt: die entstehende Si-K $\alpha$-Strahlung wird entweder durch den Detektor direkt als Si-K $\alpha$-Peak erfasst oder, falls die Si-K $\alpha$-Strahlung den Detektor verlässt, als so genannter „Escape Peak“ sichtbar, der bei der um Si-K $\alpha$-verschobenen Energie des ursprünglichen charakteristischen Röntgenstrahls auftaucht (nur bei $\mathrm{Si}(\mathrm{Li})$-Detektor).

- Summen-Peaks: zwei oder mehrere charakteristische Röntgenstrahlen treffen simultan im Detektor an und werden als ein einziger Impuls registriert. Dadurch erscheint ein Peak bei aufsummierter Energie der gleichzeitig eintreffenden Röntgenstrahlen (bei Si(Li)- und HPGe-Detektoren).

Für die qualitative Elementanalyse einer unbekannten Probe werden im Energiespektrum zunächst die Peaklagen ermittelt und mit einer Datenbank verglichen. Im Idealfall kann man an mehreren charakteristischen Röntgenpeaks $(\mathrm{K} \alpha, \mathrm{K} \beta, \mathrm{L} \alpha, \mathrm{L} \beta)$ die Elemente eindeutig identifizieren.

Zur Ermittlung der quantitativen Elementanteile wird zunächst ein Modell für den Untergrund berechnet, der größtenteils durch die Bremsstrahlung gebildet wird, und dann die Intensitäten der einzelnen Peaks aufsummiert. Aus der Gesamtintensität der einzelnen Reflexe kann schließlich die Konzentration der Elemente unter Berücksichtigung einiger Korrekturfaktoren berechnet werden. Da die rein rechnerisch bestimmten Korrekturfaktoren nur eine Näherung darstellen, empfiehlt es sich bei der energiedispersiven Analyse (EDX) für die zu untersuchenden Elemente geeignete Standardproben auszuwählen und vorher zu messen, um daraus präzise Korrektur- 
faktoren zu erhalten. Unter der Verwendung von Standardproben kann eine Genauigkeit von ca. $1 \mathrm{Gew} . \%$ erreicht werden, während sie bei der standardfreien Analyse nur $\approx 10 \mathrm{Gew} . \%$ beträgt.

\section{Analyse mit einem wellenlängendispersiven Detektor}

Im Gegensatz zur EDX werden bei der wellenlängendispersiven Analyse (WDA) die charakteristische Röntgenstrahlen zunächst in einem Fokussierungskreis („Rowland-Kreis“, Abb. 2) über einen Monochromator in Johansson-Geometrie gebündelt und gemäß der Braggschen Beugungsgleichung (Vgl. Kap. 2.2) in Richtung Detektor reflektiert. Da der d-Wert für einen Monochromator konstant ist, kann mit einem Kristall nur ein bestimmter Wellenlängenbereich abgedeckt werden. Um einen größeren Wellenlängenbereich erfassen zu können, werden daher mehrere Monochromator-Kristalle eingesetzt. Standardmäßig werden in der Mikrosonde die Kristalle „LiF“ (LiF 200, 2d = 4.028 $)$ ), „PET“ (Pentaerythrit 002, 2d = 8.742 $\AA$ ) und „TAP“ (Thalliumphthalat 101, 2d = 25,75 $\AA$ ) eingesetzt. Als Detektor wird ein Ar(Methan)-Proportionalzähler (Totzeit von $3 \mu \mathrm{s}$ ) verwendet. Die optimalen Zählraten für diesen Detektortyp liegen unter $5 \cdot 10^{4} \mathrm{cps}$.

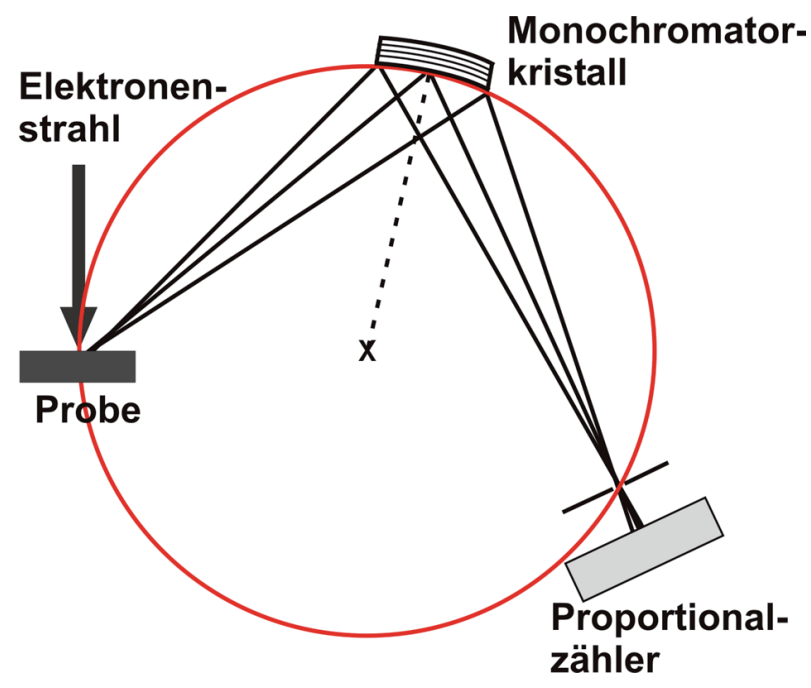

Abb. 2: Fokussierung der Röntgenstrahlen mit dem Rowland-Kreis (rot) : Dadurch dass Probe, Monochromator und Detektor auf einem Fokussierungskreis liegen, wird für eine Monochromatorposition ein konstanter Bragg-Winkel erhalten und somit nur die gewünschte Wellenlänge detektiert. Der Durchmesser des Rowland-Kreises beträgt in der Regel $140 \mathrm{~mm}$. Zusätzlich werden oft auch Spektrometer mit einem Rowland-Kreis mit einem kleinerem Durchmesser von $100 \mathrm{~mm}$ verwendet, wodurch die Energieauflösung zwar erhöht, der detektierbare Wellenlängenbereich jedoch verringert wird (diese Spektrometer werden durch das Kürzel H für ,high sensivity“ gekennzeichnet). 
Folgende Schritte sind für die quantitative Elementanalyse notwendig:

- Bestimmung der Peakmitte an einem Standard.

- Setzen von zwei Untergrundpunkten vor und nach den Peaks der charakteristischen Röntgenstrahlung für die lineare Interpolation des Untergrundes.

- Messung der Peakintensität auf dem Standard, dessen Matrix ähnlich der zu untersuchenden Probe sein sollte.

- Eventuell Berechnung von Überlappungsfaktoren einzelner Elemente.

- Messung der zu untersuchenden Probe bei gleichem Elektronenfluss wie bei der Standardprobe.

- Berechnung der Elementkonzentration.

Die Konzentration eines Elementes A ergibt sich nach der "Castaing-Näherung" (Gleichung 3), wobei die so berechneten Elementkonzentrationen noch auf Matrixeffekte korrigiert werden müssen.

$$
C_{A, \text { Probe }}=\frac{I_{A, \text { Probe }}}{I_{A, \text { Std }}} C_{A, \text { Std }}
$$

mit $C_{A, \text { Probe }}=$ Konzentration des Elements $\mathrm{A}$ in der Probe, $C_{A, S t d}=$ Konzentration des Elements A im Standard, $I_{A, \text { Probe }}=$ Peakintensität des Elementes A in der Probe und $I_{A, S t d}=$ Peakintensität des Elementes A im Standard.

Als Matrix-Korrektur wird oft die $Z A F$-Korrektur verwendet, in die im Wesentlichen drei Effekte eingehen:

- Z: Auswirkung der mittleren Ordnungszahl Z auf die Menge der Rückstreuelektronen (beinhaltet auch den Energieverlust der abgebremsten Elektronen)

- A: Absorptionskorrekturfaktor

- F: Fluoreszenzkorrekturfaktor.

\section{Vor- und Nachteile von EDX und WDA}

Aus den unterschiedlichen Detektionsverfahren ergeben sich für REM/EDX und EMS/WDA jeweils andere Vor- und Nachteile. Am REM, mit dem man sehr gute orts- und tiefenaufösende $\mathrm{SE} / \mathrm{RE}$-Bilder erzielen kann, ermöglicht die EDX-Analyse es, eine schnelle Übersicht über die Hauptbestandteile einer Probe zu erhalten (simultane Messung), wobei die eindeutige Elementidentifizierung besonders von Elementen mit hoher Ordnungszahl und ähnlicher Masse schwer fallen kann. Mit der WDA dagegen wird die Messzeit deutlich verlängert (sequentielle Messung), 
wobei jedoch aufgrund der deutlich höheren Energieauflösung die Elemente sicherer und ihre Konzentrationen genauer bestimmt werden können.

\subsection{Quantitative Phasenanalyse}

\section{Erzeugung von Röntgenstrahlen an konventionellen Röntgengeräten und am Synchrotron}

Bei bekannter Kristallstruktur von Mineralphasen in einem Kompositmaterial kann röntgenographisch ihr jeweiliger Phasenanteil bestimmt werden. Röntgenstrahlen können entweder konventionell mit einer Röntgenröhre (Abb. 3) oder in einem Teilchenbeschleuniger (Synchrotron, Abb. 5) erzeugt werden. Die Entstehung, Intensität und Energie der Röntgenstrahlung in einer Röntgenröhre unterscheiden sich dabei von denen der Synchrotronstrahlung.

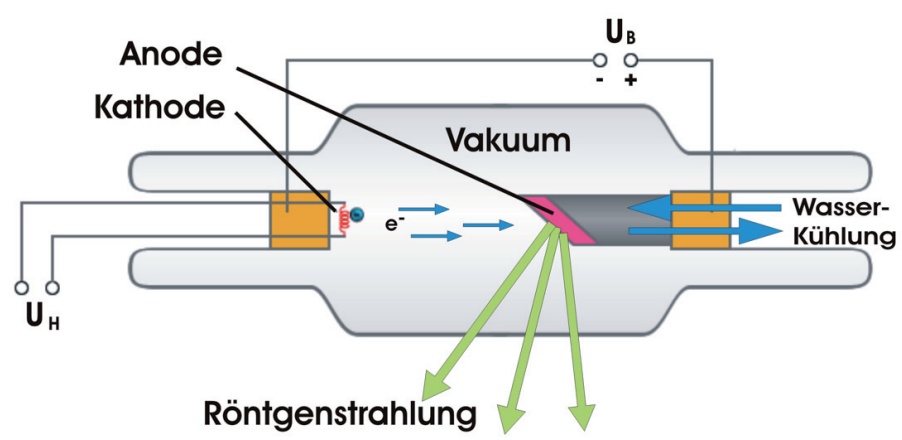

Abb. 3: Schematischer Aufbau einer Röntgenröhre. Durch die angelegte Spannung $\mathrm{U}_{H}$ wird die Kathode zum Glühen gebracht, wobei Elektronen emittieren, die durch ein elektrisches Feld zur Anode beschleunigt werden. Beim Auftreffen der Elektronen auf die Anode entstehen Röntgenstrahlung (s. Text) und sehr viel Wärme, weshalb die Anode ständig mit Wasser gekühlt werden muss.

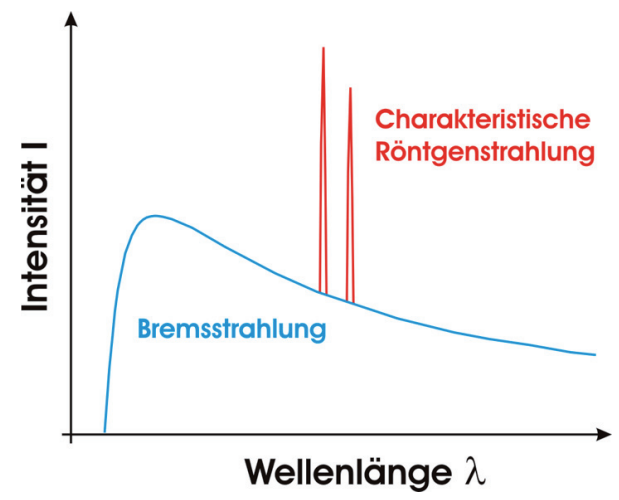

Abb. 4: Spektrum einer Röntgenröhre, die sich aus der Bremsstrahlung (blau) und der charakteristischen Strahlung (rot) zusammensetzt. 
In einer Röntgenröhre (Abb. 3) werden Elektronen aus einem Kathodenmaterial herausgelöst und durch ein elektrisches Feld auf ein Anodenmaterial (z.B. Cu, Mo, Co) beschleunigt. Beim Auftreffen der Elektronen auf die Anode wird ein Teil der Elektronen abgelenkt und gebremst, wobei ein kontinuierliches Röntgenspektrum (Bremsspektrum) entsteht. Ein anderer Teil der Elektronen schlägt kernnahe Elektronen des Anodenmaterials heraus. Diese Lücken auf den kernnahen Orbitalen werden wiederum durch andere Elektronen von höher energetischen Niveaus aufgefüllt, wobei für das Anodenmaterial charakteristische Röntgenstrahlen ausgesendet werden (Abb. 4, siehe auch Kapitel 2.1).

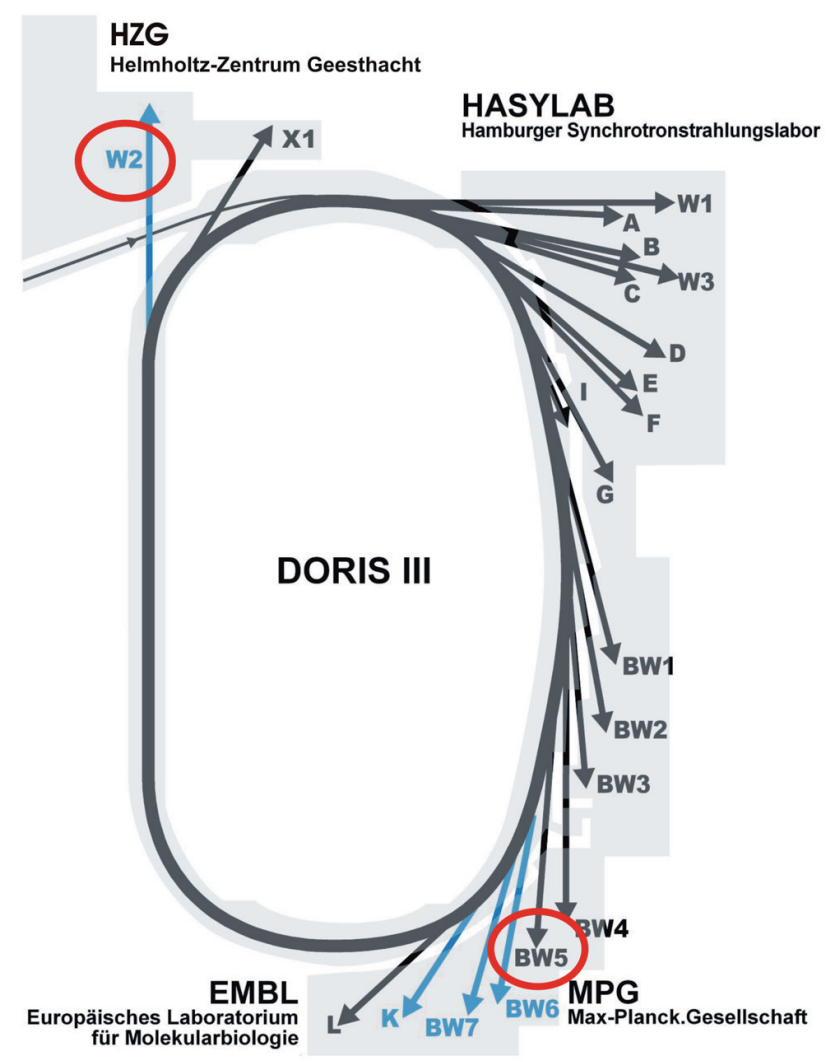

Abb. 5: Schematische Darstellung des Speicherrings DORIS III. Die Beamlines BW5 (HASYLAB) und W2 (GKSS), an denen ein Großteil der Messungen durchgeführt wurde, sind rot markiert (Quelle: hasylab.desy.de).

In einer Synchrotronquelle (s. Abb. 5) werden Elektronen oder Positronen in einen Speicherring eingespeist und beschleunigt. Dadurch dass die Teilchen durch Magnete auf eine Kreisbahn gezwungen werden, strahlen sie ein kontinuierliches und sehr intensives Bremsspektrum ab. Mit Hilfe von Kollimatoren und Monochromatoren kann eine beliebige, monochromatische Wellenlänge aus dem Bremsspektrum gefiltert werden. 


\section{Beugung von Röntgenstrahlen}

Nach Bragg (1913) kann die Beugung von Röntgenstrahlen an einem Kristallgitter als einfache Reflexion von Röntgenwellen aufgefasst werden. Der Röntgenstrahl fällt im so genannten Glanzwinkel $\theta$ ein und wird im selben Winkel wieder reflektiert (Abb. 6).

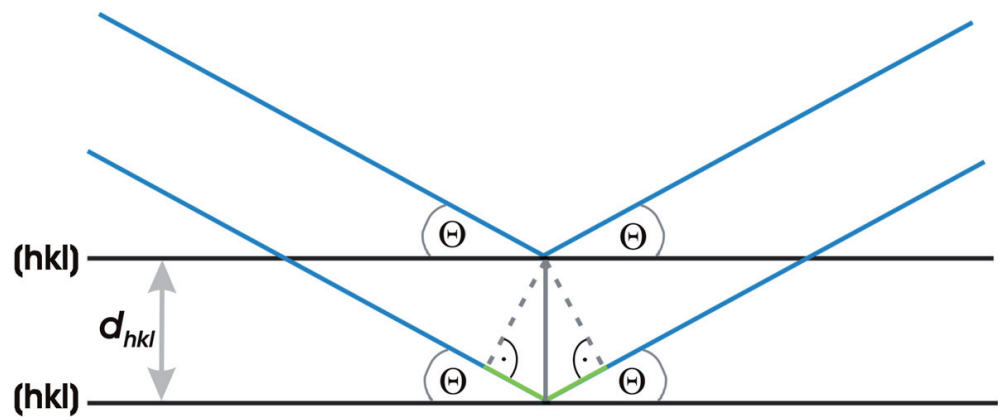

Abb. 6: Beugung der Röntgenstrahlen am Kristallgitter. Der Röntgenstrahl trifft im Glanzwinkel $\theta$ auf eine Netzebenenschar (hkl) mit Abstand $d_{h k l}$ und wird bei Erfüllung der Braggschen Gleichung reflektiert. Der Gangunterschied $2 d \sin \theta$ ist grün markiert.

Zur konstruktiven Interferenz, d.h. Beugung einer Röntgenwelle an einer Netzebene, kommt es jedoch nur, wenn die Röntgenwellen an den Netzebenen so reflektiert werden, dass sie in gleicher Phase schwingen. Gegenphasige Interferenzen löschen sich aus. Die Reflexionsbedingung ist genau dann erfüllt, wenn der Gangunterschied ein Vielfaches $n$ der Wellenlänge $\lambda$ ausmacht. Aus dieser Interferenz-Bedingung und den geometrischen Gegebenheiten (Abb. 6) ergibt sich die Braggsche Gleichung:

$$
2 d \sin \theta=n \lambda
$$

Da der Gangunterschied auch vom Netzebenenabstand $d$ abhängt, sind die Beugungswinkel $2 \theta$ charakteristisch für die Kristallstruktur des untersuchten Materials.

\section{Rietveldmethode}

Mit Hilfe der Rietveld-Methode (Rietveld, 1967) ist es möglich, die Anteile von Mineralphasen eines Kompositmaterials anhand eines Pulverdiffraktogramms zu bestimmen. Dabei wird ein berechnetes Röntgendiffraktogramm dem tatsächlich gemessenen iterativ angepasst. In die Berechnung des simulierten Röntgendiffraktogramms gehen neben den Kristallstrukur- und materialspezifischen Faktoren auch Parameter aus dem Messaufbau ein. Die Gesamtintensität eines Bragg-Reflexes setzt sich aus folgenden Parametern zusammen:

$$
I_{h k l}=K \times M \times L P \times A \times O \times\left|F_{h k l}\right|^{2}
$$




$\begin{array}{ll}K & \text { Skalierungsfaktor } \\ M & \text { Flächenhäufigkeitsfaktor } \\ L P & \text { Lorentz-Polarisationsfaktor } \\ A & \text { Absorptionskorrekturfaktor } \\ O & \text { Texturfaktor } \\ \left|F_{h k l}\right|^{2} & \text { Strukturfaktor }\left(F_{h k l}=\text { Strukturamplitude }\right) .\end{array}$

Der Skalierungsfaktor $K$, Lorentz-Polarisationsfaktor $L P$ und Absorptionskorrekturfaktor $A$ hängen vom Geräteaufbau und teilweise von den Probeneigenschaften ab, während die Informationen über die Kristallstruktur in der Strukturamplitude $F_{h k l}$ enthalten sind. Die Strukturamplitude wird hauptsächlich durch die Atomsorten, die Atompositionen, die Besetzungsgrade der Atompositionen und die Auslenkung der Atome von ihrer Ideallage bestimmt:

$$
\mathbf{F}_{h k l}=\sum_{j=1}^{n} g^{j} t^{j} f^{j}(s) e^{2 \pi \mathbf{i}\left(h x^{j}+k y^{j}+l z^{j}\right)}
$$

$g^{j} \quad$ Besetzungsfaktor, $0 \leq g^{j} \leq 1$

$t^{j}=e^{-B_{i s o}^{j} \frac{\sin ^{2} \theta}{\lambda^{2}}} \quad$ Auslenkungsfaktor mit dem atomaren, isotropen Auslenkungsparameter $B_{i s o}^{j}$ $x^{j}, y^{j}, z^{j} \quad$ Fraktionelle Atomkoordinaten

$f^{j}(s) \quad$ Atomformfaktor als Funktion von $s=\sin \theta / \lambda$ (Röntgenstrahlen),

Neutronenstreufaktoren sind unabhängig von $s$.

Der Atomformfaktor $f^{j}(s)$ führt in Röntgendiffraktogrammen zu einer kontinuierlichen Abnahme der Reflex-Intensitäten mit zunehmendem 20-Winkel. Da der atomare Streufaktor $f^{j}(s)$ eines Elements sehr von der Elektronenanzahl abhängt, wird er auf das Streuvermögen eines isolierten Elektrons normiert:

$$
f_{0}^{j}(\sin \theta / \lambda)=c_{0}^{j}+\sum_{i=1}^{4} a_{i}^{j} \exp \left(-b_{i}^{j} \sin \theta / \lambda\right)
$$

mit den elementspezifischen Koeffizienten $c_{0}^{j}, a_{1-4}^{j}$ und $b_{1-4}^{j}$.

Zudem streuen Elemente mit höherer Ordnungszahl zunehmend anomal. Diese anomale Streuung kann durch zwei zusätzliche Parameter (reale $\Delta f^{j \prime}$ und imaginäre $\mathbf{i} \Delta f^{j \prime \prime}$ Komponenten des anomalen Streufaktors) berücksichtigt werden:

$$
f^{j}(\sin \theta / \lambda)=f_{0}^{j}(\sin \theta / \lambda)+\Delta f^{j \prime}+\mathbf{i} \Delta f^{j \prime \prime}
$$

Auch die Auslenkung der Atome von ihrer Ideallage aufgrund thermischer Schwingungen oder statistischer Fehlordnung bewirkt eine Abnahme der Intensitäten mit steigendem Beugungswinkel. Der Auslenkungsparameter $B_{\text {iso }}^{j}$ beschreibt die atomare, isotrope Verschiebung eines Atoms von seiner Gleichgewichtslage. In der Literatur wird anstelle des $B_{i s o}^{j}$ häufig das mittlere Verschiebungsquadrat $U_{i s o}=\bar{u}_{i s o}^{2}$ eines Atoms von der Ideallage verwendet (Gleichung 9). 


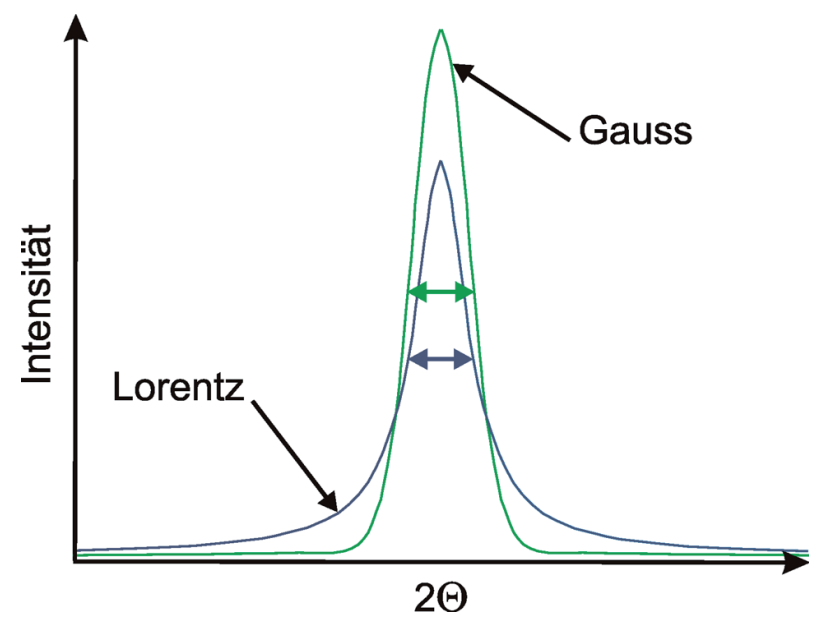

Abb. 7: Die Profilfunktionen nach Gauss (grün) und Lorentz (lila) im Vergleich (nach Pecharsky \& Zavalij, 2005).

$$
B_{i s o}^{j}=8 \pi^{2}\left(\bar{u}_{i s o}^{2}\right)^{j}
$$

Das Profil der Bragg-Reflexe wird durch die Art der Strahlungsquelle, der Probenbeschaffenheit und den Messaufbau bestimmt. Oft ist die Beschreibung der Bragg-Reflexe mit nur reinen Gauß- oder Lorentzfunktionen (Funktionen $G(x)$ und $L(x)$ in Gleichung 10 und 11 sowie Abb. 7) unzureichend und es empfiehlt sich eine Kombination dieser beiden Funktionen zu nehmen. Da eine Faltung dieser Funktionen zu rechenaufwendig wäre, werden sie in den meisten Rietveld-Programmen linear kombiniert (Pseudo-Voigt-Funktion, Gleichung 12).

$$
\begin{aligned}
& G(x)=\frac{C_{G}^{1 / 2}}{\sqrt{\pi} H} \exp \left(-C_{G} x^{2}\right) \\
& L(x)=\frac{C_{L}^{1 / 2}}{\pi H^{\prime}}\left(1+C_{L} x^{2}\right)^{-1}
\end{aligned}
$$

$$
\begin{aligned}
& G(x) \\
& L(x) \\
& x=\left(2 \theta_{i}-2 \theta_{k}\right) / H_{k}
\end{aligned}
$$

$$
\begin{array}{ll}
C_{G} & =4 \ln 2 \\
C_{L} & =4 \\
H & =\sqrt{U \tan ^{2} \theta+V \tan \theta+W} \\
H^{\prime} & =L X / \cos \theta+L Y \tan \theta
\end{array}
$$

\section{Gauß-Funktion}

\section{Lorentz-Funktion}

Bragg-Winkel des i-ten Punkts im Röntgendiffraktogramm in Bezug auf den 2 $\theta$-Wert des k-ten BraggReflexes, geteilt durch die Halbwertsbreite dieses Reflexes bei halbem Intensitätsmaximum (FWHM: full width at half maximum).

mit $U, V$ und $W$ als variable Profilparameter.

mit $L X$ und $L Y$ als Lorentz-Profilparameter. 


$$
y(x)=\eta \overbrace{\frac{C_{G}^{1 / 2}}{\sqrt{\pi} H} \exp \left(-C_{G} x^{2}\right)}^{\text {Gauss }}+(1-\eta) \overbrace{\frac{C_{L}^{1 / 2}}{\pi H}\left(1+C_{L} x^{2}\right)^{-1}}^{\text {Lorentz }}
$$

mit $\eta=\eta_{0}+\eta_{1} 2 \theta+\eta_{2} 2 \theta^{2}$ als Mischungskoeffizient $\left(\eta_{0}, \eta_{1}\right.$ und $\eta_{2}$ sind freie Variable) und der Halbwertsbreite $H=\sqrt{U \tan ^{2} \theta+V \tan \theta+W}$.

Der Untergrund eines Röntgen- oder Synchrotrondiffraktogramms wird durch verschiedene Faktoren beeinflusst, wobei der Messaufbau sowie die Probenbeschaffenheit eine wichtige Rolle spielen. Am Besten lässt er sich durch ein Polynom beschreiben, dessen Anzahl an Termen je nach Komplexität des Untergrunds variiert werden kann.

Die Güte der Profilanpassung kann zum einen optisch im Vergleich des berechneten mit dem gemessenen Röntgendiffraktogramm erfasst werden und zum anderen auch mathematisch durch die Gütekriterien $R_{w p}$ (gewichteter R-Wert, Gleichung 13) und GOF (Goodness-of-Fit, Gleichung 14) berechnet werden.

$$
\begin{aligned}
& R_{w p}=\sqrt{\frac{\sum w_{i}\left(y_{i o}-y_{i c}\right)^{2}}{\sum w_{i} y_{i o}^{2}}} \\
& G O F=\frac{\sum w_{i}\left(y_{i o}-y_{i c}\right)^{2}}{n-p+c}
\end{aligned}
$$

mit den Wichtungsfaktoren $w_{i}$, der experimentellen $y_{i o}$ und berechneten $y_{i c}$ Intensität an einem Punkt $i$, der Gesamtanzahl an gemessenen Datenpunkten $n$, Anzahl der freigegebenen LeastSquares-Parameter $p$ und Anzahl der gekoppelten Parameter $c$.

Im Idealfall sollte der gewichtete R-Wert am Ende einer Rietveldverfeinerung $<0,1$ und der GOF zwischen 2 und 4 sein. Da in die Berechnung des $R_{w p}$ und des GOF auch die Anpassung des Untergrunds eingeht, ist es wichtig, besonders bei Röntgendiffraktogrammen mit einem relativ hohen Untergrund, die Profilanpassung auch optisch zu kontrollieren, da ein gut angepasster Untergrund über schlecht angepasste Bragg-Reflexe hinwegtäuschen kann.

Für jeden verfeinerten Parameter werden in den Rietveld-Programmen geschätzte Standardabweichungen ( $\underline{\text { estimated }} \underline{\underline{s}}$ tandard $\underline{\text { deviation }}=$ e.s.d.) angegeben. In diesen e.s.d.s ist nur der rein rechnerische Fehler enthalten. Nicht berücksichtigt sind systematische und experimentelle Fehler sowie die Korrelation von Fehlern.

\subsubsection{Bestimmung des Glasanteils mittels röntgenographischer Methoden}

Röntgenographisch kann der Glasanteil eines unbekannten Pulvers entweder unter Zusatz eines Standardminerals oder unter der Annahme eines Strukturmodells für die Glasphase bestimmt werden: 
- Bestimmung des Glasanteils mit Hilfe eines internen Standards: Der pulverisierten Probe wird ein möglichst vollständig kristalliner Standard hinzugefügt, sodass das fertige Pulvergemisch 10 Gew.\% des Standards enthält. Dieses Pulver kann für die quantitative Rietveld-Analyse entweder konventionell in Reflektion oder in Transmission gemessen werden. Anhand der aus der Rietveld-Analyse berechneten Menge des Standards $R_{S}$ und der Einwaage $X_{S}$ kann nun der amorphe Phasenanteil $X_{A}$ nach Gualtieri (1999) bzw. Torre et al. (2001) wie folgt berechnet werden (alle Angaben in Gew.\%):

$$
X_{A}=\frac{1-X_{S} / R_{S}}{100-X_{S}} \cdot 10^{4} \%
$$

Hierbei muss beachtet werden, dass die Summe aller Phasen $\left(X_{i}\right)$ im System inklusive des Glasanteils $\left(X_{A}\right)$ und des Standards $\left(X_{S}\right) 100 \%$ entsprechen: $\left(\sum_{i} X_{i}\right)+X_{A}+X_{S}=100 \%$.

- Modellierung der amorphen Phase durch eine scheinkristalline Struktur: Die amorphe Phase wird durch eine kristalline Mineralphase modelliert, deren Nahordnung der des Glases entspricht. Das Fehlen der Fernordnung, typisch für amorphe Substanzen, kann dann dadurch simuliert werden, dass in der Rietveld-Verfeinerung die Kristallitgröße in derselben Größenordnung wie die Elementarzelle gewählt wird. Im Fall von reinem $\mathrm{SiO}_{2}$-Glas wurde die Glas-Struktur nach Le Bail (1995) sehr gut durch eine $\alpha$-Carnegieit-Struktur mit der orthorhombischen Raumgruppe $R G=P 2_{1} 2_{1} 2_{1}$ beschrieben. Dieses Modell wurde durch Lutterotti et al. (1998) zur Bestimmung des $\mathrm{SiO}_{2}$-Glasanteils in Keramiken mit dem Textur- und Rietveld-Programm MAUD erfolgreich eingesetzt, wobei in dieser Arbeit das kubische Strukturmodell mit der Raumgruppe $R G=P 2_{1} 3$ für den $\alpha$-Carnegieit angenommen wurde. Auf diese Art sollten auch Anteile anderer Gläser in Glaskeramiken bestimmbar sein, wobei der kritischste Punkt während der Rietveld-Analyse die Wahl des für das Glas geeigneten Strukturmodells ist.

\subsection{Textur und anisotrope Eigenschaften von polykristallinen Materialien}

Unter Textur versteht man im mineralogischen bzw. materialwissenschaftlichen Sinne die Orientierungsverteilung aller in einem polykristallinen Material vorhandenen Kristallite. Da die einzelnen Kristallite oft anisotrope physikalische Eigenschaften besitzen, ist ihre Orientierung auch für die anisotropen physikalischen Eigenschaften der gesamten Probe ausschlaggebend. Die Berechnung der Textur sowie der anisotropen physikalischen Eigenschaften einer Probe ist für einphasige, polykristalline Materialien wie z.B. reine Korundplättchen oder Reinmetall-Bleche erprobt. Auch für mehrphasige, polykristalline Materialien wie z.B. Stahllegierungen werden Texturuntersuchungen industriell oft durchgeführt. In einigen Fällen wird sogar bewusst eine bestimmte Texturart und -stärke in einem Werkstoff hergestellt, um so seine Eigenschaften optimal den Einsatzbedingungen anzupassen.

Zur Beschreibung der Texturstärke wird die vorhandene Textur mit der Textur einer Probe mit statistisch verteilten Kristalliten verglichen: Statistisch verteilte Orientierungen werden als einfach regellos bezeichnet. Je mehr Kristallite in einer bestimmten Orientierung vorkommen, desto höher regellos (zweifach, dreifach etc.) ist die Textur. Wenn fast alle Kristallite in einer einzigen Orientierung vorkommen, spricht man von einem Einkristall. 


\subsubsection{Darstellung von Orientierungen}

Die Beschreibung der Textur erfolgt, indem die Orientierung der Kristallite in Bezug auf die Gesamtprobe dargestellt wird. Die Orientierung jedes einzelnen Kristallits kann auf verschiedene Weise beschrieben werden. Dafür wird jedem Kristallit ein eigenes Koordinatensystem $K_{B}$ und der Probe ein weiteres Koordinatensystem $K_{A}$ zugewiesen. Die Wahl des Koordinatensystems für die Kristallite ergibt sich aus der Kristallsymmetrie, während das Probenkoordinatensystem frei wählbar ist. Für rechteckige Proben würde sich z.B. eine orthorhombische Probensymmetrie anbieten, wobei die Achsen meist als LR (Längsrichtung, X), QR (Querrichtung, Y) und NR (Normalrichtung, Z) bezeichnet werden.

Die Orientierung $g$ wird durch die Überführung des Probenkoordinatensystems $K_{A}$ auf das Kristallkoordinatensystem $K_{B}$ erreicht. Da die relative Orientierung der Kristallite zur Probengeometrie für die Texturbeschreibung ausreicht, ist nur eine Rotation und keine Translation nötig (Gleichung 16).

$$
K_{B}=g \cdot K_{A}=a_{i j} \cdot K_{A}
$$

mit $a_{i j}=\left(\begin{array}{lll}a_{11} & a_{12} & a_{12} \\ a_{21} & a_{22} & a_{22} \\ a_{31} & a_{32} & a_{33}\end{array}\right)$ als Rotationsmatrix.

Eine weitere anschaulichere Methode, die Orientierung $g$ darzustellen, ist die Angabe der Kristallfläche (hkl) parallel zur Probenoberfläche (NR) und der in Längsrichtung (LR) liegenden Gitterrichtung [uvw] (Gleichung 17).

$$
g=(h k l)[u v w]
$$

Die Orientierung $(h k l)[u v w]$ beschreibt dabei eine Ideallage, die von den meisten Kristalliten in einer Probe eingenommen wird. Mit dieser Bezeichnung wird jedoch nicht darauf eingegangen, wie vielfach regellos die Textur der vorliegenden Probe ist. Für die quantitative Texturanalyse eignet sich daher folgende Darstellungsmöglichkeit besser: Die Orientierung $g$ wird nun nicht nur durch die Ideallage oder durch eine einzige Rotationsmatrix beschrieben, sondern durch drei Einzelrotationen, den Eulerschen Drehungen, um jeweils eine Achse des Probenkoordinatensystems $K_{A}$ (Abb. 8):

1. Drehung um Z-Achse von $K_{A}: \rightarrow \phi_{1}$-Drehung,

2. Drehung um neue X-Achse von $K_{A}: \rightarrow \Phi$-Drehung,

3. Drehung um neue Z-Achse von $K_{A}: \rightarrow \phi_{2}$-Drehung.

Die Drehwinkel um die jeweilige Achse werden als Eulerwinkel $\phi_{1}, \Phi$ und $\phi_{2}$ bezeichnet (Abb. 8) und die Orientierung $g$ kann durch $g=\left\{\phi_{1}, \Phi, \phi_{2}\right\}$ beschrieben werden. Die Reihenfolge der Drehungen und Festlegung der Drehachsen wird in Europa, wie hier vorgestellt, nach der Bunge-Konvention durchgeführt (Bunge, 1993). Andere Konventionen beziehen sich auf eine andere Reihenfolge der Drehungen bzw. andere Wahl der Drehachsen, sind aber leicht ineinander überführbar. 


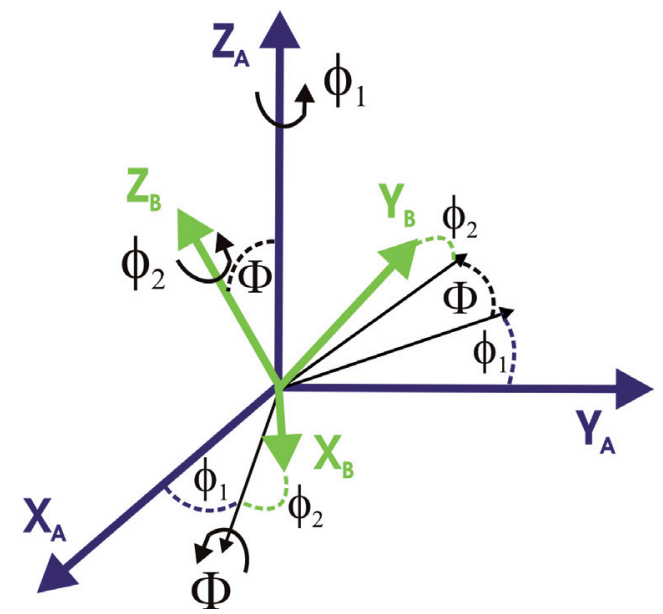

Abb. 8: Definition der Eulerwinkel. Abgebildet sind das der Probe zugeordnete Koordinatensystem $K_{A}$ mit den Achsen $X_{A}, Y_{A}$ und $Z_{A}$ sowie ein Beispiel für ein Kristallkoordinatensystem $K_{B}$ mit den Achsen $X_{B}, Y_{B}$ und $Z_{B}$. Zudem sind die Eulerwinkel der Einzelrotationen eingetragen (Abb. nach Bunge, 1993).

Für mathematische Berechnungen und die quantitative Texturanalyse werden die drei Einzelrotationen durch Multiplikation der Einzelmatrizen zu einer Rotationsmatrix zusammengefasst (Gleichung 18).

$$
\begin{aligned}
g & =\left\{\phi_{1}, \Phi, \phi_{1}\right\} \\
& =\left(\begin{array}{rrr}
\cos \phi_{1} & \sin \phi_{1} & 0 \\
-\sin \phi_{1} & \cos \phi_{1} & 0 \\
0 & 0 & 1
\end{array}\right) \cdot\left(\begin{array}{rrr}
1 & 0 & 0 \\
0 & \cos \Phi & \sin \Phi \\
0 & -\sin \Phi & \cos \Phi
\end{array}\right) \cdot\left(\begin{array}{rrr}
\cos \phi_{2} & \sin \phi_{2} & 0 \\
-\sin \phi_{2} & \cos \phi_{2} & 0 \\
0 & 0 & 1
\end{array}\right) \\
& =\left[\begin{array}{rrr}
\cos \phi_{1} \cos \phi_{2}-\sin \phi_{1} \sin \phi_{2} \cos \Phi & \sin \phi_{1} \cos \phi_{2}+\cos \phi_{1} \sin \phi_{2} \cos \Phi & \sin \phi_{2} \sin \Phi \\
-\cos \phi_{1} \sin \phi_{2}-\sin \phi_{1} \cos \phi_{2} \cos \Phi & -\sin \phi_{1} \sin \phi_{2}+\cos \phi_{1} \cos \phi_{2} \cos \Phi & \cos \phi_{2} \sin \Phi \\
& -\sin \phi_{1} \sin \Phi &
\end{array}\right] \\
& =\left[\begin{array}{lll}
u & r & h \\
v & s & k \\
w & t & l
\end{array}\right]
\end{aligned}
$$

Im hexagonalen Kristallsystem ist die Beziehung zwischen der Orientierungsmatrix $g\left\{\phi_{1}, \Phi, \phi_{2}\right\}$ und hkil $\langle u v t w\rangle$ durch folgende zwei Gleichungen gegeben (Wang \& Huang, 2003):

$$
\left[\begin{array}{c}
h \\
k \\
i \\
l
\end{array}\right]=\left[\begin{array}{ccc}
\frac{\sqrt{3}}{2} & -\frac{1}{2} & 0 \\
0 & 1 & 0 \\
-\frac{\sqrt{3}}{2} & -\frac{1}{2} & 0 \\
0 & 0 & c / a
\end{array}\right]\left[\begin{array}{c}
\sin \phi_{2} \sin \Phi \\
\cos \phi_{2} \sin \Phi \\
\cos \Phi
\end{array}\right]
$$

und 


$$
\left[\begin{array}{c}
u \\
v \\
w \\
t
\end{array}\right]=\left[\begin{array}{ccc}
\frac{2}{3} & -\frac{1}{3} & 0 \\
0 & \frac{2}{3} & 0 \\
-\frac{2}{3} & -\frac{1}{3} & 0 \\
0 & 0 & c / a
\end{array}\right] \times\left[\begin{array}{c}
\cos \phi_{1} \cos \phi_{2}-\sin \phi_{1} \sin \phi_{2} \cos \Phi \\
-\cos \phi_{1} \sin \phi_{2}-\sin \phi_{1} \cos \phi_{2} \cos \Phi \\
\sin \phi_{1} \sin \Phi
\end{array}\right]
$$

\subsubsection{Berechnung und Darstellung von Polfiguren}

Polfiguren sind graphische Projektionen zur Darstellung von Poldichteverteilungen einer Netzebenenart (hkl) relativ zum Probenkoordinatensystem. Zur Beschreibung der Orientierung eines Kristalls relativ zum Probenkoordinatensystem wird die Lage einer beliebigen Kristallrichtung $\vec{h}=[h k l]$ durch die Polarkoordinaten $\theta$ und $\gamma$ beschrieben (Abb. 9a), während die Orientierung der Probe relativ zum Kristallkoordinatensystem durch eine beliebige Probenrichtung $\vec{y}$ und mit Hilfe der Polarkoordinaten $\alpha$ und $\beta$ dargestellt werden kann (Abb. 9b). In beiden Fällen wird durch die Polarkoordinaten nur jeweils eine Kristall- bzw. Probenrichtung beschrieben. Dadurch ist die exakte Lage des Kristall- bzw. Probenkoordinatensystems nicht festgelegt, weil eine Rotation um die jeweilige Achse $\vec{h}$ bzw. $\vec{y}$ noch möglich ist. Der freie Rotationswinkel wird als $\psi$ bezeichnet. Mathematisch kann die Polfigur durch eine Achsenverteilungsfunktion erfasst werden (Gleichung 21), wobei über alle Rotationswinkel $\psi$ integriert wird.

$$
A(\vec{h}, \vec{y})=\frac{1}{2 \pi} \int_{\vec{h} \| \vec{y}} f(g) d \psi
$$

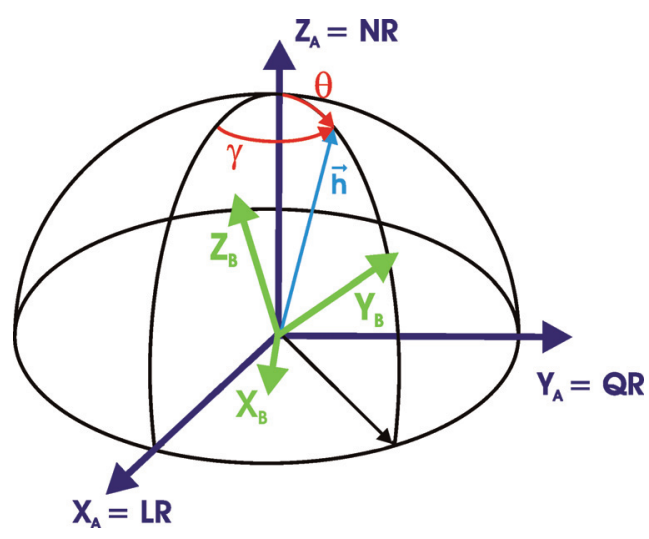

(a) Darstellung einer beliebigen Kristallrichtung $\vec{h}$ durch die Polarkoordinaten $\theta$ und $\gamma$.

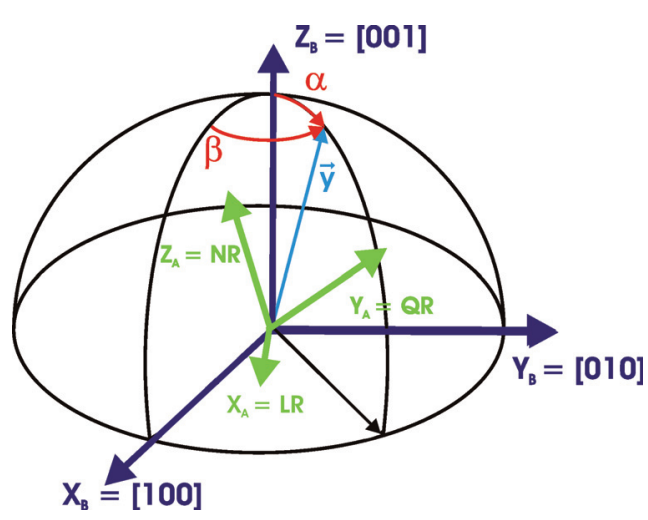

(b) Darstellung einer beliebigen Probenrichtung $\vec{y}$ durch die Polarkoordinaten $\alpha$ und $\beta$.

Abb. 9: Definition der Polarkoordinaten. Abgebildet sind jeweils das der Probe zugeordnete Koordinatensystem $K_{A}$ mit den Achsen $X_{A}, Y_{A}$ und $Z_{A}$ sowie das Kristallkoordinatensystem $K_{B}$ mit den Achsen $X_{B}, Y_{B}$ und $Z_{B}$ (Bsp. für kubische Kristallstruktur; modifizierte Abbildung nach Bunge, 1993).

Je nachdem welcher Richtungsvektor konstant gehalten wird, kann nun eine normale Polfigur ( $\vec{h}=$ konst., zeigt Probensymmetrie $)$ oder eine inverse Polfigur $(\vec{y}=$ konst., zeigt Kristallsymmetrie) berechnet werden. In der Regel werden normale Polfiguren gemessen, wobei die Verteilung 
einer Netzebenenschar relativ zum Probenkoordinatensystem betrachtet wird (Gleichung 22).

$$
A\left(\vec{h}_{i}, \vec{y}\right)=P\left(\vec{h}_{i}, \vec{y}\right)=P_{\vec{h} i}(\alpha, \beta)=\frac{1}{2 \pi} \int_{(\vec{h} \| \vec{y}) \perp(\alpha, \beta)} f(g) d \psi
$$

mit $\vec{h}_{i}=$ const

Aufgrund der Kristallsymmetrie überlagern sich bei zentrosymmetrischen Kristallstrukturen die positiven und negativen $h k l$-Reflexe vollständig und auch bei nicht-zentrosymmetrischen Kristallstrukturen ist bei Anwendung konventioneller Texturmessverfahren aufgrund des Friedelschen Gesetzes eine Unterscheidung von $h k l$ und $\bar{h} \bar{k} \bar{l}$ nicht möglich. Daher sind die gemessenen Polfiguren i.d.R. zwischen $\vec{h}$ und $-\vec{h}$ gemittelte Polfiguren (Gleichung 23).

$$
\widetilde{P}_{\vec{h} i}(\alpha, \beta)=1 / 2\left[P_{\vec{h} i}(\alpha, \beta)+P_{-\vec{h} i}(\alpha, \beta)\right]
$$




\subsubsection{Orientierungsverteilungen}

Die Orientierungsverteilung von Kristalliten in einer Probe lässt sich allgemein in einer Volumenbzw. Kristallitanzahl-Dichtefunktion darstellen (Gleichung 22 und 25).

$$
\begin{aligned}
& \frac{d V}{V}=f(g) d g \\
& \frac{d N}{N}=n(g) d g
\end{aligned}
$$

Hierbei stehen $d V$ und $d N$ für alle Volumenelemente bzw. alle Kristallite mit der Orientierung $g$ und $V$ bzw. $N$ für das Gesamtvolumen bzw. die Gesamtanzahl aller Kristallite der Probe. Im Fall einer regellosen Textur wird ihre Orientierungsverteilungsfunktion $f_{r}$ auf 1 normiert (Gleichung 26); das bedeutet, dass jede beliebige Orientierungsverteilungsfunktion $f(g)$ auf ein Vielfaches der regellosen Textur bezogen wird (im Engl. abgekürzt mit „mrd“ für „multiples of random distribution").

$$
f_{r}=\oint f(g) d g=1
$$

Zur Berechnung der Orientierungsverteilungsfunktion (ODF - engl. für „orientation distribution function“) gibt es verschiedene Ansätze, von denen zwei im Folgenden vorgestellt werden sollen:

- Texturanalyse unter Anwendung von symmetrischen verallgemeinerten Kugelfunktionen (Bunge, 1969)

- diskrete E-WIMV-Methode (Lutterotti et al. (2004); eine Erweiterung (engl. extension) des WIMV-Algorithmus nach den Entwicklern benannt: Williams-Imhof-Matthies-Vinel; Matthies \& Vinel (1982))

\subsubsection{Berechnung der Orientierungsverteilung mit Hilfe von symmetrischen verallgemeinerten Kugelfunktionen}

Die Orientierungsverteilung $f(g)$ lässt sich in einer Reihe nach verallgemeinerten Kugelfunktionen entwickeln. Da sowohl die Probe als auch die Kristalle oft Symmetrieeigenschaften aufweisen, ist es sinnvoll diese direkt in die Berechnung einfließen zu lassen (Gleichung 27).

$$
f(g)=\sum_{l=0}^{\infty} \sum_{\mu=1}^{M(l)} \sum_{\nu=1}^{N(l)} C_{l}^{\mu \nu} \dot{\vec{T}}_{l}^{\mu \nu}(g)
$$


$l \quad$ Reihenentwicklungsgrad, $l_{\max }$ wird je nach Kristall- und Probensymmetrie auf Werte zwischen 6 und 22 gesetzt

$M(l) \quad$ Zahl der unabhängigen Funktionen der Kristallsymmetrie

$N(l) \quad$ Zahl der unabhängigen Funktionen der Probensymmetrie

$C_{l}^{\mu \nu} \quad$ Texturkoeffizient

$\dot{\vec{T}}_{l}^{\mu \nu}(g) \quad$ symmetrische verallgemeinerte Kugelfunktionen

Die Symmetrieeigenschaften der Probe und der Kristallite sind durch einen einfachen Punkt (rechts - Probensymmetrie) bzw. einen Doppelpunkt (links - Kristallsymmetrie) über dem Symbol der symmetrischen verallgemeinerten Kugelfunktionen $\dot{\overparen{T}}_{l}^{\mu \nu}(g)$ gekennzeichnet.

Ziel der Texturanalyse ist die Gleichung $27 \mathrm{zu}$ lösen und die $C_{l}^{\mu \nu}$-Koeffizienten zu berechnen. Die Berechnung erfolgt nicht direkt, sondern in einem Least-Squares-Verfahren, indem Informationen aus den experimentellen Polfiguren ausgewertet werden (vgl. Abb. 10). Dazu wird zunächst einmal die Achsenverteilungsfunktion $A(\vec{h}, \vec{y})$ betrachtet (Gleichung 28).

$$
A(\vec{h}, \vec{y})=\frac{1}{2 \pi} \int_{\vec{h} \| \vec{y}} f(g) d \psi=\frac{1}{2 \pi} \sum_{l=0}^{L} \sum_{\mu=0}^{M(l)} \sum_{\nu=0}^{N(l)} \frac{4 \pi}{2 l+1} C_{l}^{\mu \nu} \cdot \ddot{k}_{l}^{\mu}(\vec{h}) \cdot \dot{k}_{l}^{\nu}(\vec{y})
$$

$\dot{k}_{l}^{\nu}(\vec{y}) \quad$ symmetrische Kugelflächenfunktion der Probensymmetrie, $\ddot{k}_{l}^{\mu}\left(\overrightarrow{h_{i}}\right) \quad$ symmetrische Kugelflächenfunktion der Kristallsymmetrie.

Im nächsten Schritt wird die Kristallrichtung festgehalten:

$$
P_{\vec{h}}(\vec{y})=\sum_{l=0}^{\infty} \sum_{\nu=1}^{N(l)} F_{l}^{\nu}(\vec{h}) \dot{k}_{l}^{\nu}(\vec{y})
$$

mit den F-Koeffizienten:

$$
F_{l}^{\nu}(\vec{h})=\frac{4 \pi}{2 l+1} \sum_{\mu=1}^{M(l)} C_{l}^{\mu \nu} \ddot{k}_{l}^{\mu}\left(\overrightarrow{h_{i}}\right)
$$

Aus jeder Polfigur können dann die experimentellen F-Koeffizienten $F_{l}^{\nu}(\vec{h})_{\exp }$ wie folgt berechnet werden:

$$
F_{l}^{\nu}(\vec{h})_{e x p}=\frac{1}{2 \pi} \iint P_{\vec{h}}(\alpha \beta) \cdot \dot{k}_{l}^{\nu}(\alpha \beta) \cdot \sin \alpha \cdot d \alpha d \beta
$$

Die Texturkoeffizienten $C_{l}^{\mu \nu}$ werden schließlich im Least-Squares-Verfahren mit Hilfe der folgenden Minimierungsfunktion berechnet, wobei die Größen $F_{l}^{\nu}(\vec{h})_{\text {exp }}$ und $\ddot{k}_{l}^{\mu}(\vec{h})$ bekannt sind:

$$
\sum_{i}\left[F_{l}^{\nu}(\vec{h})_{\text {exp }}-\frac{4 \pi}{2 l+1} \sum_{\mu=1}^{M(l)} C_{l}^{\mu \nu} \ddot{k}_{l}^{\mu}(\vec{h})\right]^{2}=\text { Min }
$$




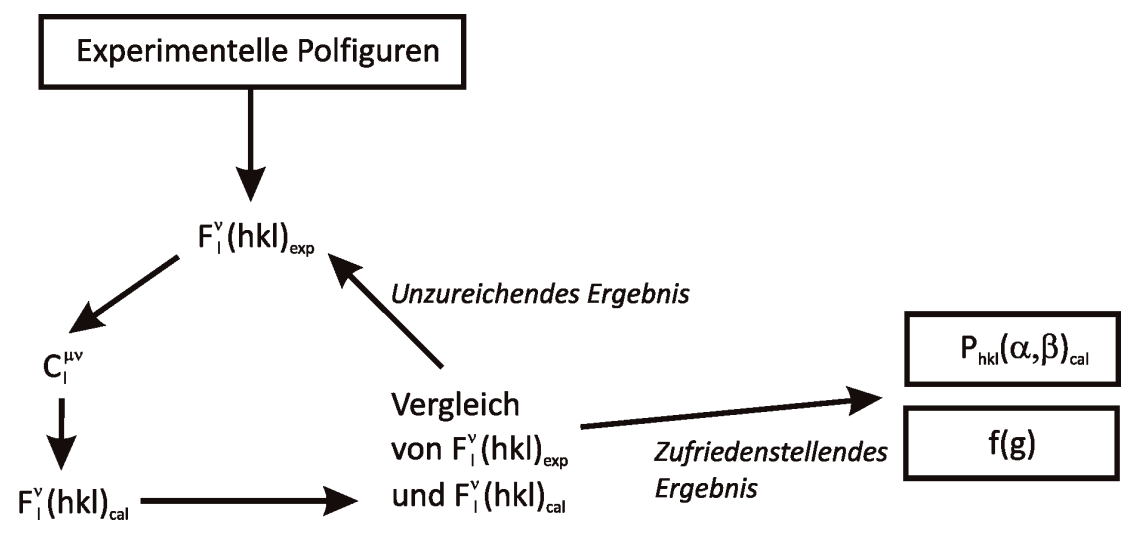

Abb. 10: Flussdiagrammm zur Berechnung der ODF und Polfiguren nach der Methode der symmetrischen verallgemeinerten Kugelfunktionen. Hierbei sind $F_{l}^{\nu}(\vec{h})_{\exp }$ die experimentellen F-Koeffizienten, $F_{l}^{\nu}(\vec{h})_{c a l}$ die berechneten F-Koeffizienten und $P_{\vec{h}}(\alpha \beta)_{c a l}$ die zurück berechneten Polfiguren.

Zur Überprüfung der Güte einer Texturberechnung ist es immer sinnvoll, die experimentellen $P_{h k l}(\alpha, \beta)_{\text {exp }}$ mit den zurückberechneten Polfiguren $P_{h k l}(\alpha, \beta)_{\text {cal }}$ zu vergleichen $\left(\Delta P_{h k l}(\alpha, \beta)=\right.$ $\left.P_{h k l}(\alpha, \beta)_{e x p}-P_{h k l}(\alpha, \beta)_{c a l}\right)$. Ein weiteres Güte-Kriterium, das bei dieser Methode angewendet werden kann, ist die Entwicklung der absoluten Mittelwerte der $C_{l}^{\mu \nu}$-Koeffizienten und ihr Fehler in Abhängigkeit vom Reihenentwicklungsgrad $l$. Mit zunehmendem Reihenentwicklungsgrad $l$ sollten beide Werte kleiner werden und gegeneinander konvergieren.

Die graphische Darstellung von $f(g)$ kann in ebenen Schnitten und in gleichmäßigen Abständen durch den Eulerraum (aufgespannt durch $\phi_{1}, \Phi$ und $\phi_{2}$ ) erfolgen, wobei meist $\phi_{2}$ konstant gehalten wird. Die Poldichteverteilung wird dabei in Isolinien angezeigt (Abb. 11).

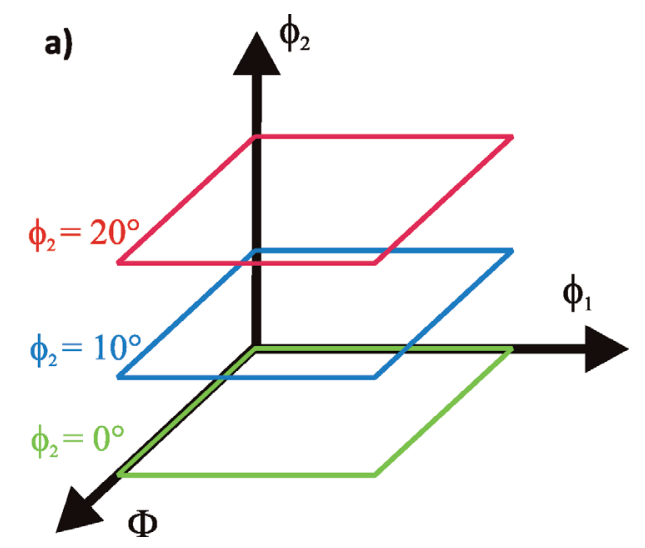

b)

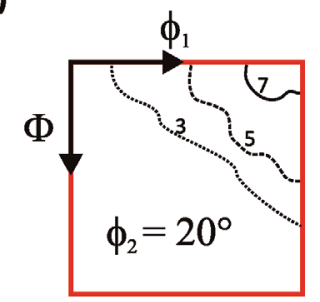

Abb. 11: Darstellung der Orientierungsverteilung $f(g)$ im Eulerraum.

(a) Eulerraum mit $\phi_{2}$-Schnitten

(b) Beispiel für einen Schnitt mit $\phi_{2}=20^{\circ}$ 


\subsubsection{Berechnung der Orientierungsverteilung nach der E-WIMV-Methode}

Die E-WIMV-Methode ist eine Weiterentwicklung der WIMV-Methode (Matthies \& Vinel, 1982; Lutterotti et al., 2004; Chateigner, 2010), bei der die Orientierungsverteilung direkt aus den experimentellen Polfiguren berechnet wird. Dafür werden die Polfiguren nach einem frei wählbaren Raster (z.B. $5^{\circ}$ ) in Zellen aufgeteilt. Jede dieser Zelle liegt entlang einer oder mehrerer Projektionslinien innerhalb der ODF und der Wert einer Polfigurenzelle $p_{\vec{h}}(\vec{y})$ entspricht dem Mittelwert der Werte aus den entsprechenden Zellen in der ODF:

$$
p_{\vec{h}}(\vec{y})=\frac{1}{N} \sum_{i=1}^{N} f\left(\vec{y} \Leftarrow \vec{g}_{i}\right)
$$

$\vec{g} \quad$ Kristallorientierung

$\vec{y} \quad$ Punkt auf der Polfigur mit der Kristallrichtung $\vec{h}$

$N$ Zahl der Orientierungszellen $\overrightarrow{g_{i}}$ der ODF, die zur Poldichte der Zelle $\vec{y}$ beitragen

Die Lösung dieser Gleichung erfordert die Inversion einer sehr großen Matrix und ist daher im Vergleich zur konventionellen Texturberechnung nach Bunge (1993) sehr rechenintensiv. Die erste Schätzung der Orientierungsverteilung $f_{0}(\vec{g})$ wird erreicht, indem für jede Zelle der ODF das geometrische Mittel der entsprechenden Polfigurzellen als Startwert genommen wird (Gleichung $34)$.

$$
f_{0}(\vec{g})=N_{0} \prod_{j=1}^{I} \prod_{m_{i}=1}^{M_{i}} p_{\overrightarrow{h_{i}}}^{e x p}\left(y_{m_{i}}\right)^{\frac{1}{I M_{i}}}
$$

I Zahl der experimentellen Polfiguren

$M_{i} \quad$ Häufigkeit des i-ten Pols

$N_{0} \quad$ Normierungsfaktor

Im Weiteren wird $f(g)[\mathrm{ODF}]$ iterativ neu berechnet, wobei aus dem Vergleich der Werte für eine n-te zurückberechnete Polfigur $p_{n}$ (aus der n-ten Schätzung der ODF berechnet) mit der entsprechenden experimentellen Polfigur ein Korrekturfaktor berechnet wird (vgl. Abb. 12; Gleichung 35). Oft konvergiert der WIMV-Algorithmus schnell, sodass eine zufriedenstellende Lösung der ODF meist mit 10 bis 12 Iterationen erreicht wird (Kocks et al., 2000).

$$
f^{n+1}(g)=N_{n} \frac{f^{n}(g) f_{0}(g)}{\left(\prod_{j=1}^{I} \prod_{m_{i=1}}^{M_{i}} p_{\overrightarrow{h_{i}}}^{\exp }\right)^{\frac{1}{I M_{i}}}}
$$

$f^{n+1}(g) \quad n+1$-te Schätzung der ODF

$f^{n}(g) \quad n$-te Schätzung der ODF

$N_{n} \quad$ Normierungsfaktor für $n$-te Schätzung der ODF

Die Erweiterung der WIMV-Methode zur E-WIMV-Methode besteht darin, dass $f(g)$ im Vergleich zur WIMV-Methode - ähnlich wie bei einer weiteren diskreten Texturmethode (ADCMethode, engl. für ,arbitrarily-defined cells") - geglättet wird. Bei der ADC-Methode werden 


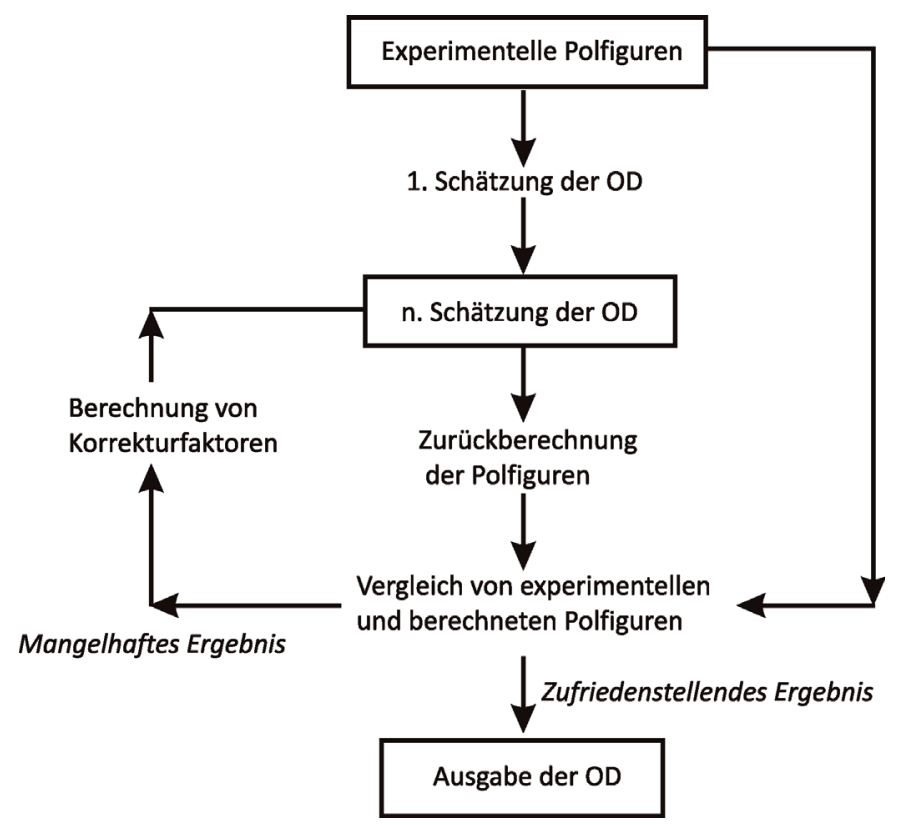

Abb. 12: Flussdiagrammm des WIMV-Algorithmus (modifizierte Abbildung nach Kocks et al., 2000). Orientierungsdichte wird hier mit OD abgekürzt.

zur Informationsübertragung aus den gemessenen Polfiguren in $f(g)$ statt Projektionslinien Projektionsröhren verwendet (Chateigner, 2010). Außerdem werden die Werte für $f(g)$ nach einem Entropie-Iterations-Algorithmus berechnet, wobei die Reflexe gewichtet werden (Gleichung 36; Chateigner (2010)).

$$
f^{n+1}(g)=f^{n}(g) \prod_{m_{i=1}}^{M_{i}}\left(\frac{p_{\overrightarrow{h_{i}}}}{p_{\overrightarrow{h_{i}}}^{n}}\right)^{r_{n} \frac{w_{i}}{M_{i}}}
$$

$r_{n} \quad$ Relaxationsparameter; $0<r_{n}<1$

$w_{i} \quad$ Gewicht der einzelnen Reflexe (Gewichtung je nach Intensität und Überlappungsgrad)

Wie auch bei der harmonischen Methode - Berechnung der Orientierungsverteilungsfunktion mit Hilfe von symmetrischen verallgemeinerten Kugelfunktionen - lässt sich die ODF-Auflösung bei der E-WIMV-Methode steuern. Während nach der harmonischen Methode die Auflösung der Textur durch Variation im Reihenentwicklungsgrad $l$ (niedriges $l$ entspricht einer niedrigen Auflösung), kann die Auflösung nach der E-WIMV-Methode durch die Wahl der Zellgröße bestimmt werden (kleine Zellen entsprechen einer hohen Auflösung). 


\subsubsection{Fasertexturen}

Fasertexuren sind spezielle Texturen, bei denen eine hohe Orientierungsdichte entlang einer Richtung im Eulerraum vorliegt $\left(\phi_{1}, \Phi, \phi_{1}\right)$. Ihre Orientierungsverteilung ist axialsymmetrisch. Die Ideallage solcher Fasertexturen kann durch $g=(h k l)$ ausreichend beschrieben werden, da der Term $[u v w]$ beliebige Werte annehmen kann und somit keine Aussagekraft besitzt (vgl. Gleichung 17). Ein weiteres Merkmal von Fasertexturen ist, dass sie bei röntgenographischen 2dBeugungsbildern keine Intensitätsmaxima auf ihren Beugungsringen zeigen, wenn der Strahl parallel zur Faserachse verläuft (Abb. 13). Die Fasertexturen entstehen oft bei Herstellungsprozessen wie Drahtziehen, Strangpressen, Sedimentation oder Axialdruck.
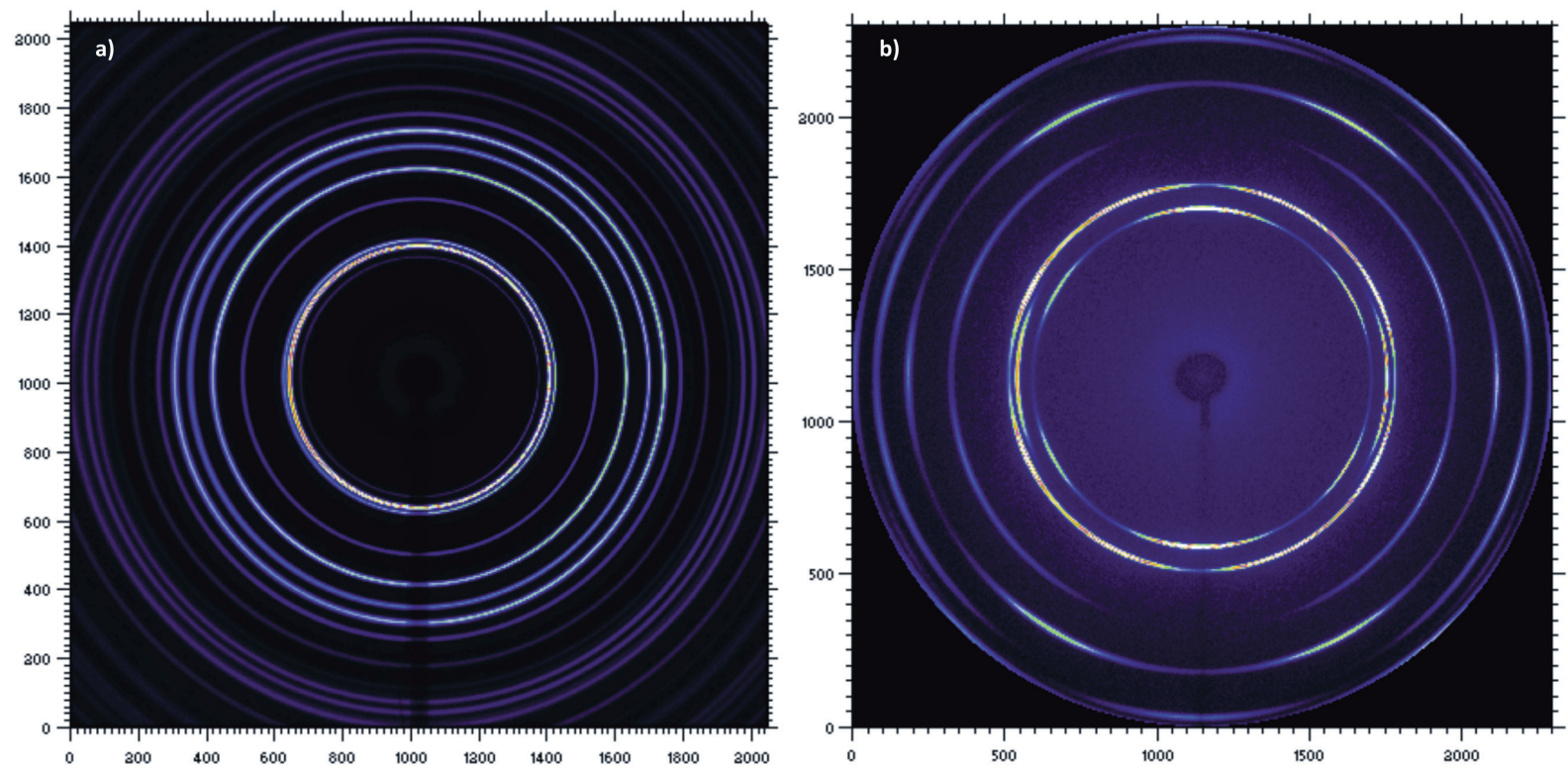

Abb. 13: Beispiel für Fasertexturen: 2d-Beugungsbilder von einem Titanwurzelstift der Firma Gebr. Brasseler. In (a) ist der Wurzelstift in Längsrichtung und in (b) in Radialrichtung zum Strahl orientiert. Die Fasertextur bewirkt, dass in (a) - im Gegensatz zu (b) - keine Intensitätsmaxima auf den Beugungsringen zu sehen sind, sodass die Kristallite dieser Probe zunächst als regellos orientiert erscheinen. Beamline BW5 $\left(\lambda_{(a)}=0,1243 \AA, \lambda_{(b)}=0,1613 \AA\right)$.

\subsubsection{Berechnung von makroskopischen physikalischen Eigenschaften unter Berücksichtigung der Textur}

Die makroskopischen, anisotropen Eigenschaften einer polykristallinen, einphasigen Probe können mit dem Wissen um die Orientierungsverteilung der Kristallite bei bekannten anisotropen physikalischen Eigenschaften eines Einkristalls derselben Phase berechnet werden. Eine beliebige physikalische Eigenschaft einer polykristallinen Probe kann z.B. als ein mit der Orientierung gewichteter Eigenschaftswert $\bar{S}_{i j k l}$ der entsprechenden Einkristalleigenschaft $S_{i j k l}$ dargestellt werden (Gleichung 37). 


$$
\bar{S}_{i j k l}=\oint S_{i j k l}(g) \cdot f(g) d g
$$

Allerdings muss berücksichtigt werden, dass die Reaktion der Kristallite auf einen äußeren Einfluss nicht nur von ihrer eigenen Kristallorientierung, sondern auch von ihrer Größe, Form und Anordnung sowie von der Orientierung, Größe und Form ihrer Nachbarn abhängt. Als Beispiel sei hier das Hooksche Gesetz aufgeführt (Gleichung 38):

$$
\bar{\epsilon}_{i j}=\tilde{S}_{i j k l} \cdot \bar{\sigma}_{k l}
$$

$\bar{\epsilon}_{i j} \quad$ anisotrope, makroskopische Dehnung

$\tilde{S}_{i j k l} \quad$ wahre Steifigkeit $\left(\neq \bar{S}_{i j k l}\right)$

$\bar{\sigma}_{i j} \quad$ anisotrope, makroskopische Spannung

Der wahre Wert der Steifigkeit $\tilde{S}_{i j k l}$ kann zwei Extremfälle annehmen:

- Kristallite sind plattenförmig angeordnet und die Spannung ist konstant (Reuss, 1929); Ausgangspunkt: $\epsilon_{i j}=S_{i j k l}(g) \cdot \bar{\sigma}_{k l}$

- Kristallite sind stabförmig angeordnet und die Dehnung ist konstant (Voigt, 1928);

Ausgangspunkt: $\sigma_{i j}=C_{i j k l}(g) \cdot \bar{\epsilon}_{k l}, C_{i j k l}=\left[S_{i j k l}\right]^{-1}\left(C_{i j k l}\right.$ als E-Modul)

Die dazugehörigen Mittelwerte erhält man, wenn das Hooksche Gesetz in der entsprechenden Form in Gleichung 37 eingesetzt wird (Gleichung 39a, 39b).

$$
\begin{aligned}
& \bar{S}_{i j k l}^{\text {Reuss }}=\oint S_{i j k l}(g) \cdot f(g) d g \\
& \bar{C}_{i j k l}^{\text {Voigt }}=\oint C_{i j k l}(g) \cdot f(g) d g
\end{aligned}
$$

Da diese Extremfälle in der Regel nicht der Fall sind, wird als Näherung für den wahren Wert der Mittelwert aus beiden Berechnungen verwendet (Hill, 1952). Als Beispiel ist in Gleichung 40 die Mittelung für den E-Modul angegeben.

$$
\bar{C}_{i j k l}^{\text {Hill }}=1 / 2\left\{\bar{C}_{i j k l}^{\text {Voigt }}+\left[\bar{S}_{i j k l}^{\text {Reuss }}\right]^{-1}\right\}
$$

Auf ähnliche Weise können weitere anisotrope Eigenschaften wie z.B. die Wärmeausdehnung einer polykristallinen Probe berechnet werden. 


\subsection{Ermittlung der Vickershärte und des E-Moduls mit dem instrumentellen Eindringverfahren}

Zur Ermittlung der Vickershärte und des elastischen Moduls wurde das instrumentierte Eindringverfahren nach Oliver \& Pharr (1992) verwendet. Allgemein wird Härte als der Widerstand definiert, den ein Material einem eindringenden Gegenstand entgegensetzt. Zur Bestimmung der Vickershärte wird als Eindringkörper eine pyramidale Diamantspitze mit einem Öffnungswinkel von $136^{\circ}$ verwendet. Die Vickershärte $H V$ wird anhand der Größe des plastischen Eindrucks wie folgt berechnet:

$$
H V=\frac{F}{A_{\text {Vickers }}} \approx 0,1891 \cdot \frac{F}{d^{2}}
$$

mit $F=$ Prüflast, $A_{V i c k e r s}=$ Oberfläche des Eindrucks und $d=$ Mittelwert der zwei Diagonalen.

Je nach Größe der verwendeten Prüflast und der zu erwartenden Eindringtiefe wird das VickersVerfahren in vier Bereiche eingeteilt:

$\begin{array}{ll}\text { Makro-Härteprüfung } & \text { Prüfkraft }>49,03 \mathrm{~N} \\ \text { Kleinlast-Härteprüfung } & \text { Prüftkraft 1,961-49,03 N } \\ \text { Mikro-Härteprüfung } & \text { Prüfkraft }<1,961 \mathrm{~N} \\ \text { Nano-Härteprüfung } & \text { Eindringtiefe }<0,2 \mu \mathrm{m}\end{array}$

Bei dem hier verwendeten instrumentierten Eindringverfahren wird jedoch nicht die Größe des Eindrucks direkt gemessen, sondern nur die Tiefe der eindringenden Diamantspitze in Abhängigkeit von der aufgebrachten Kraft. Aus dem gemessen Tiefenwert und dem Wissen über die Geometrie des Indenters kann auf die Eindrucksfläche zurückgeschlossen werden. Allerdings setzt sich die maximale Tiefe des Eindrucks $h$ aus der plastischen und elastischen Verformung zusammen $\left(h=h_{c}+h_{e}\right.$, s. Abb. 14a), während sich die Vickershärte nur auf die plastische Verformung bezieht. Daher muss die elastische Komponente beim instrumentierten Eindringverfahren von der maximal erreichten Tiefe abgezogen werden. Als Korrekturfaktor für den Anteil der elastischen Verformung geht der Anfangsentlastungsanstieg $S=\left(\frac{d F}{d h}\right)_{h=h \max }$ (s. Abb. 14b) in die Berechnung der Eindringhärte $H_{I T}$ wie folgt ein:

$$
H_{I T}=\frac{F_{\max }}{A_{p}\left(h_{c}\right)}
$$

mit $h_{c}=h_{\max }-\frac{\epsilon F_{\max }}{S}, A_{p}\left(h_{c}\right)$ als projizierte Oberfläche zur Höhe $h_{c}$ und $\epsilon$ als Korrekturfaktor je nach Eindringkörpergeometrie ( $\epsilon=0,75$ für eine Vickers-Pyramide).

Aus der Eindringhärte kann für viele Materialien die Vickershärte in guter Näherung mit $H V=$ $0,0945 \cdot H_{I T}$ bestimmt werden (Weiler, 1990). In der Regel wird die Vickershärte in der Form „xxx HV y/z" angegeben. An der Stelle xxx steht der gemessene Härtewert, y gibt Auskunft über die verwendete Prüflast $[\mathrm{kp}]$ und z über die Einwirkdauer [s]. Wenn eine Einwirkdauer von 10-15s verwendet wird, dann kann diese Angabe auch wegfallen. In neuerer Zeit wird der Härtewert oft in der SI-Einheit MPa ohne Auskunft über Prüflast und Einwirkdauer angegeben. Der Zusammenhang zwischen diesen Größen ist durch [HV] 9,807 = [ ] MPa hergestellt. 
a)

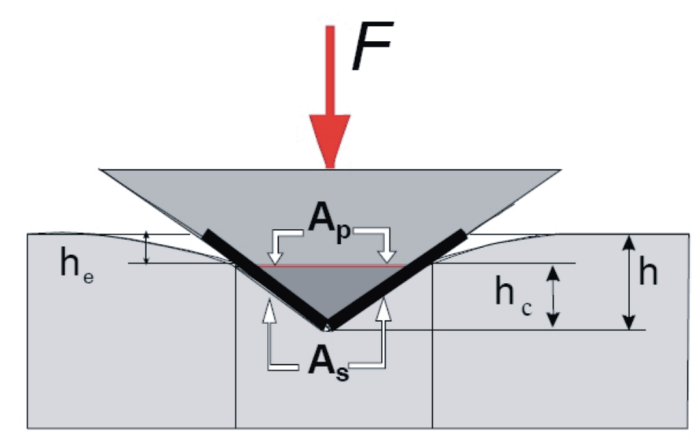

b)

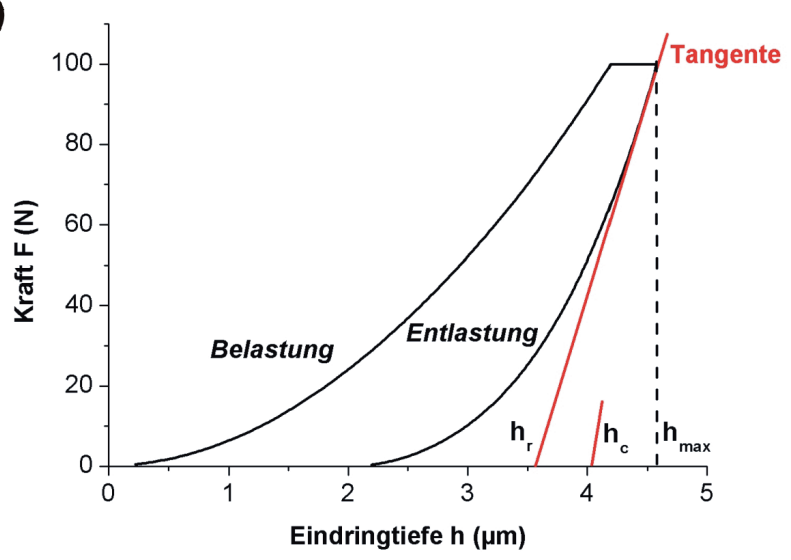

Abb. 14: Prinzip des instrumentierten Eindringverfahrens (Abb. nach Ullner, 2004).

(a) Geometrische Beziehungen der verschiedenen Eindringtiefen durch elastische $\left(h_{e}\right)$ und plastische $\left(h_{c}\right)$ Verformung sowie der betrachteten Eindrucksfläche $A_{p}$.

(b) Typische Kraft-Eindringtiefenkurve $(100 \mathrm{mN} / 20 \mathrm{~s})$ mit Haltezeit $(20 \mathrm{~s})$. Die rot eingezeichnete Tangente hat die Steigung $S=\left(\frac{d F}{d h}\right)_{h=h \max }$ und wird zur Berechnung des elastischen Eindringmoduls herangezogen.

Neben der Vickershärte ist es auch möglich, mithilfe des Anfangsentlastungsanstieges $S$ den elastischen Eindringmodul $E_{I T}$ aus einer Härtemessung zu ermitteln:

$$
E_{I T}=\frac{1-\nu_{s}^{2}}{\frac{1}{E_{r}}-\frac{1-\nu_{i}^{2}}{E_{i}}}
$$

\footnotetext{
$\nu_{s} \quad$ Poisson-Zahl der Probe

$\nu_{i} \quad$ Poisson-Zahl des Indenters $(0,07)$

$E_{r}=\frac{\sqrt{\pi} S}{2 \sqrt{A_{p}\left(h_{c}\right)}} \quad$ reduzierter Eindringmodul

$E_{i} \quad$ elastischer Modul des Indenters (1140 GPa)
} 


\section{Material}

Die Eigenschaften von Zahnmaterialien müssen auf die unterschiedlichen Anwendungsbereiche der Zahnmedizin optimiert sein. Das bedeutet, dass je nach Indikation andere direkte und indirekte Füllmaterialien bzw. Zahnersatzwerkstoffe eingesetzt werden müssen. Im Bereich der direkten Füllmaterialien werden als Anwendungsgebiete die Kavitätenklassen nach Black (1917) unterschieden:
Klasse I Grübchen und Fissuren der Kauflächen
Klasse II approximale Kavitäten an Prämolaren und Molaren
Klasse III approximale Frontzahnkavitäten
Klasse IV approximale Frontzahnkavitäten mit Verlust der Schneidekantenecke
Klasse V Zahnhalskavitäten

Auf dem gegenwärtigen Markt ist eine sehr große Anzahl an unterschiedlichen Zahnmaterialien vorhanden, von denen einige im Rahmen dieser Arbeit auf ihre physikalischen Eigenschaften untersucht wurden. Um die Lesbarkeit der vorliegenden Arbeit zu erhöhen, werden im Folgenden alle eingetragenen Handelsnamen der Zahnersatzmaterialien und die Firmen durch kursive

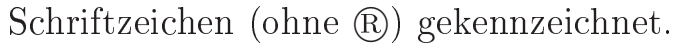

\subsection{Direkte Füllmaterialien}

\subsubsection{Amalgam}

Tab. 1: Chemische Zusammensetzung zweier Dentalamalgame der Firma Ivoclar Vivadent nach Herstellerangaben.

\begin{tabular}{lcccc}
\hline \hline Handelsname & Ag [Gew.\%] & Sn [Gew.\%] & Cu [Gew.\%] & Hg [Gew.\%] \\
\hline Amalcap regular & 35,9 & 9,3 & 6,1 & 48,8 \\
Amalcap fast & 36,4 & 9,3 & 6,1 & 48,2 \\
\hline \hline
\end{tabular}

Im Rahmen dieser Arbeit werden zwei moderne $\gamma_{2}$-freie Dentalamalgame der Firma Ivoclar Vivadent untersucht (Abb. 15, chemische Zusammensetzung s. Tab. 1). Sie härten nach Vermischen des flüssigen Quecksilbers mit dem Pulver, wie in Gleichung 2 (Abschnitt 1.2.2) beschrieben, unter Bildung der $\gamma_{1}-\mathrm{Ag}_{2} \mathrm{Hg}_{3}$ - und der $\eta$ - $\mathrm{Cu}_{6} \mathrm{Sn}_{5}$-Phase aus, wobei die Ausgangsphasen $\gamma-\mathrm{Ag}_{3} \mathrm{Sn}$ und $\mathrm{Ag}-\mathrm{Cu}$ nicht vollständig aufgebraucht werden. 
Interessant ist hierbei, ob sich alle aufgeführten Phasen tatsächlich bilden und ob durch den Stopfvorgang eine Vorzugsorientierung der Kristallite produziert wird. Die Ausgangsphase $\mathrm{Ag}_{3} \mathrm{Sn}$ $(\gamma)$ liegt in orthorhombischer (Raumgruppe $R G=\mathrm{P} m m n ; a=$ $5,968(9) \AA, b=4,7802(4) \AA$ und $c=5,1843(9) \AA$; Fairhurst \& Cohen, 1972) und die Amalgamphase $\mathrm{Ag}_{2} \mathrm{Hg}_{3}(\gamma 1)$ in kubischer Kristallstruktur vor $(R G=I 23 ; a=10,05 \AA$, (Murphy, 1931; Preston, 1931; Baird \& Muller, 1969)). Die $\eta$-Phase $\mathrm{Cu}_{6} \mathrm{Sn}_{5}$ ist hexagonal $\left(R G=\mathrm{P} 6_{3} / m m c ; a=4,192(2) \AA\right.$ und $c=5.037(2) \AA$, Gangulee et al., 1973).

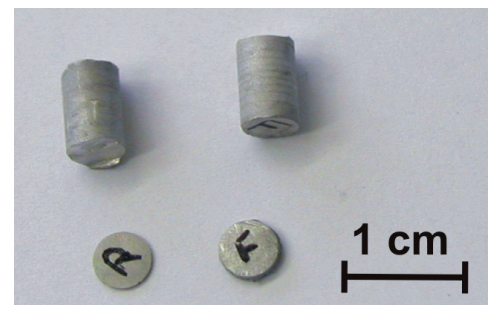

Abb. 15: Dentalamalgame Amalcap regular (links) und fast (rechts).

\subsubsection{Dentalkomposite}

Auch wenn Amalgam als einziges Langzeitfüllungsmaterial von der Krankenkasse vollständig bezahlt wird, kommen immer mehr Dentalkomposite zum Einsatz, die aufgrund ihrer besseren Ästhetik und der gesundheitlichen Bedenken gegenüber Amalgam vorgezogen werden. Eine Übersicht über die hier untersuchten Dentalkomposite (Abb. 16) und ihre Anwendungsbereiche befindet sich in Tab. 2. Die meisten der hier aufgeführten Dentalkomposite basieren auf Kunstharzen wie das aro-

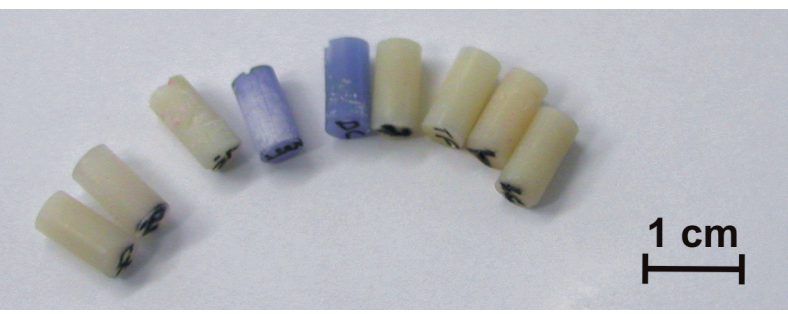

Abb. 16: Dentalkomposite (von links nach rechts): Ceram X, Clip, Venus C3, Luxacore, Rebilda DC, Tetric Evo Ceram, Tetric Evo Flow, Tetric und Rebilda LC. matische BIS-GMA (Bisphenol A-Glycidylmethacrylat) und/oder Urethan-Dimethacrylat sowie auf Füllpartikeln aus Ba-Al-Si-Glas und evtl. $\mathrm{YbF}_{3}$-Kristalliten (genaue Zusammensetzung s. Anhang Tab. A.1). Verbindungen schwerer Elemente wie $\mathrm{YbF}_{3}$ oder Gläser aus Elementen mit hoher Masse werden gerne in Dentalkompositen eingesetzt, um diesen die vorgeschriebene Röntgenopazität zu geben (Collares et al., 2010).

\section{Mineralogische Zusammensetzung der Dentalkomposite}

In den Dentalkompositen sind nur wenige kristalline Komponenten zu erwarten. In den hier vorliegenden kommt nur $\mathrm{YbF}_{3}$ als kristalline Phase vor. $\mathrm{YbF}_{3}$ hat eine orthorhombische Kristallstruktur (RG: Pnma, $a=6,218 \AA, b=6,785 \AA, c=4,431 \AA$; Bukvetskii \& Garashina, 1977). 
Tab. 2: Anwendungsbereiche und Verarbeitung der untersuchten Dentalkomposite

\begin{tabular}{|c|c|c|c|}
\hline Firma & Handelsname & Indikationen & Verarbeitung \\
\hline $\begin{array}{l}\text { Ivoclar } \\
\text { vivadent }\end{array}$ & Tetric & $\begin{array}{l}\text { Füllungen der Klassen I bis V sowie von } \\
\text { Milchzähnen, erweiterte Fissurenversiegelung }\end{array}$ & UV-härtend \\
\hline $\begin{array}{l}\text { Ivoclar } \\
\text { vivadent }\end{array}$ & Tetric Evo Flow & $\begin{array}{l}\text { Füllungen der Klassen III bis V sowie für kleinere } \\
\text { Füllungen }\end{array}$ & UV-härtend \\
\hline $\begin{array}{l}\text { Ivoclar } \\
\text { vivadent }\end{array}$ & $\begin{array}{l}\text { Tetric Evo } \\
\text { Ceram }\end{array}$ & Füllungen der Klassen I bis V & UV-härtend \\
\hline$D M G$ & Luxacore dual & Stumpfaufbau, Befestigung von Wurzelstiften & Dual-härtend \\
\hline VOCO & Rebilda $L C$ & Stumpfaufbau, Ausblockungen & UV-härtend \\
\hline VOCO & Rebilda $D C$ & $\begin{array}{l}\text { Stumpfaufbau, Befestigung von Faser-verstärkten } \\
\text { Komposit-Wurzelstiften }\end{array}$ & Dual-härtend \\
\hline Dentsply & CeramX & $\begin{array}{l}\text { Alle Kavitätenklassen im Front- und } \\
\text { Seitenzahnbereich }\end{array}$ & UV-härtend \\
\hline $\begin{array}{l}\text { Heraeus } \\
\text { Kulzer }\end{array}$ & Venus C3 & Füllungen der Klassen I bis V & UV-härtend \\
\hline VOCO & Clip & Provisorische Füllungen aller Art & UV-härtend \\
\hline$V O C O$ & $\begin{array}{l}\text { Cimara Zircon, } \\
\text { Grandioso }\end{array}$ & $\begin{array}{l}\text { Reparatur von } \mathrm{ZrO}_{2} \text {-Keramik, Füllungen der } \\
\text { Klassen I bis V }\end{array}$ & UV-härtend \\
\hline
\end{tabular}




\subsubsection{Glasionomerzemente und Zn-Sulfat-Zemente}

Für temporäre Füllungen und für Füllungen in Milchzähnen werden anstelle von Amalgam oder Dentalkompositen oft Glasionomerzemente (Haltbarkeit: ca. 3 bis 5 Jahre) oder Zn/CaSulfat-Zemente (Haltbarkeit ca. 1 bis 2 Wochen) eingesetzt.

Als Stellvertreter der Glasionomerzemente (GIZ) wird in dieser Arbeit Ketac Fil Plus der Firma 3M ESPE untersucht (Abb. 17b, Tab. 3), das wie die Dentalamalgame in Applikationskapseln vom Hersteller geliefert wird. Die Applikationskapsel wird vor der zahnärztlichen Anwendung ebenfalls in einen Schüttler eingebaut, in dem Pulver und Flüssigkeit (chemische Zusammensetzung s. Anhang Tab. A.2) vermengt werden und die chemische Härtung initiiert wird.

Von den Zinksulfatzementen werden hier zwei Vertreter untersucht (Abb. 17a, Tab. 3). Die Zinksulfatzemente werden als Paste (chemische Zusammensetzung s. Anhang Tab. A.2) geliefert und härten durch Reaktion mit Wasser bzw. Speichel aus. Für beide Zementarten stellt sich die Frage, welche chemische Reaktionen auftreten, ob diese röntgenographisch sichtbar sind und ob sich eine Vorzugsorientierung der kristallinen Phasen feststellen lässt.

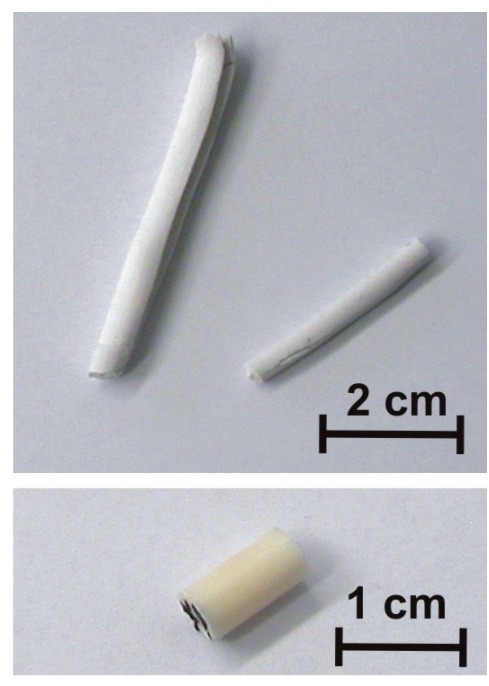

Abb. 17: a) Zinksulfatzemente Cavit (links) und Detaferm (rechts) und b) Glasionomerzement Ketac Fil Pus.

Tab. 3: Anwendungsbereiche und Verarbeitung der untersuchten provisorischen Füllmaterialien.

\begin{tabular}{|c|c|c|c|c|}
\hline Firma & Handelsname & Material & Indikationen & Verarbeitung \\
\hline $3 M E S P E$ & $\begin{array}{l}\text { Ketac } \\
\text { Plus }\end{array}$ & Glasionomerzement & $\begin{array}{l}\text { Füllungen der Klassen III } \\
\text { und V sowie kleinere Füllun- } \\
\text { gen, Milchzahnfüllungen }\end{array}$ & chemisch härtend \\
\hline Detax & Detaferm & Zn-Sulfat-Zement & Temporäre Füllungen & wasserhärtend \\
\hline $3 M E S P E$ & Cavit $W$ & Zn-Sulfat-Zement & Temporäre Füllungen & wasserhärtend \\
\hline
\end{tabular}

\section{Mineralogische Zusammensetzung der Glasionomerzemente und Zink-Sulfat-Zemente}

Der Glasionomerzement besteht hauptsächlich aus verschiedenen, amorphen Silikatglas-Verbindungen. Daher sind keine kristallinen Verbindungen darin zu erwarten.

Die Ausgangsphasen der Zinksulfatzemente sind Zinkoxid, wasserfreie bzw. leicht hydrierte Calcium-, Barium- und Zinksulfat-Verbindungen plus evtl. Talk und Calciumfluorid. Die kristallographischen Daten dieser Verbindungen sind wie folgt: 
- Zinkoxid $(\mathrm{ZnO})$ - mineralogisch Zinkit - hat eine hexagonale Kristallstruktur ( $R G: P 6{ }_{3} m c$, $a=3,24992(5) \AA, c=5,20658(8) \AA$; Albertsson et al., 1989) und ist chemisch sehr stabil.

- Der Begriff Calciumsulfat wird in der Industrie als Oberbegriff für wasserfreie und in verschiedenen Stufen hydrierte $\mathrm{CaSO}_{4}$-Verbindungen verwendet. $\mathrm{Zu}$ dieser Gruppe gehören:

- Anhydrit $\left(\mathrm{CaSO}_{4}\right)$ mit orthorhombischer Kristallstruktur ( $R G:$ Amma, $a=6,993 \AA$, $b=6,995 \AA, c=6,245 \AA$; Hawthorne \& Ferguson, 1975),

- Bassanit als Hemihydrat $\left(\mathrm{CaSO}_{4} \cdot 0,5 \mathrm{H}_{2} \mathrm{O}\right)$ mit trigonaler Kristallstruktur ( $R G$ : $P 3_{1} 21, a=6,937(2) \AA, c=6,345(1) \AA ;$ Abriel \& Nesper, 1993),

- Gips als Calciumdihydrat $\left(\mathrm{CaSO}_{4} \cdot 2 \mathrm{H}_{2} \mathrm{O}\right)$ mit monokliner Kristallstruktur $(R G$ : $C 2 / c, a=6,2840(10) \AA, b=15,2000(10) \AA, c=6,5230(12) \AA, \beta=127,414(11)$; Boeyens \& Ichharam, 2002).

- Bariumsulfat $\left(\mathrm{BaSO}_{4}\right)$, natürlich vorkommend als Baryt (Schwerspat) bekannt, ist ein schwer lösliches, nominell wasserfreies Mineral mit orthorhombischer Kristallstruktur ( $R G$ : Pnma, $a=8,848(2) \AA, b=5,441(1) \AA, c=7,132(1) \AA$; Sawada \& Takeuchi, 1990).

- Unter dem Begriff der Zinksulfate wird wie beim Calciumsulfat eine Mineralgruppe in verschiedenen Hydrierungsstufen verstanden. Es gibt u.a. folgende Zinksulfatverbindungen:

- wasserfreies Zinksulfat $\mathrm{ZnSO}_{4}$, auch Zinkosit genannt, mit orthorhombischer Kristallstruktur (RG: Pnma, $a=8,604(5) \AA, b=6,746(5) \AA, c=4,774(3) \AA$; Wildner \& Giester, 1988),

- Zinksulfat-Monohydrat (Gunningit, $\mathrm{Zn}\left(\mathrm{SO}_{4}\right) \cdot\left(\mathrm{H}_{2} \mathrm{O}\right)$ ) mit monokliner Kristallstruktur $\left(R G: C 2 / c, a=6,925(2) \AA, b=7,591(2) \AA, c=7,635(3) \AA, \beta=118.19(1)^{\circ}\right.$; Wildner \& Giester, 1991),

- Zinksulfat-Pentahydrat $\left(\left(\mathrm{Zn}(\mathrm{OH})_{2}\right)_{3} \mathrm{Zn}\left(\mathrm{SO}_{4}\right) \cdot 5\left(\mathrm{H}_{2} \mathrm{O}\right)\right)$ mit trikliner Kristallstruktur $\left(R G: \quad P \overline{1}, \quad a=8,354(2) \AA, \quad b=8,350(2) \AA, \quad c=11,001(2) \AA, \quad \alpha=94,41(2)^{\circ}\right.$, $\beta=82,95(2)^{\circ}, \gamma=119,93(2)$; Bear et al., 1986),

- Zinksulfat-Hexahydrat (Bianchit, $\mathrm{Zn}\left(\mathrm{SO}_{4}\right) \cdot 6\left(\mathrm{H}_{2} \mathrm{O}\right)$ ) mit monokliner Kristallstruktur $\left(R G: C 2 / c, a=9,981(2) \AA, b=7,250(9) \AA, c=24,280(3) \AA, \beta=98.45(7)^{\circ}\right.$; Spiess \& Gruehn, 1979),

- Zinksulfat-Heptahydrat (Goslarit oder Zinkvitriol genannt, $\mathrm{Zn}\left(\mathrm{SO}_{4}\right) \cdot 7\left(\mathrm{H}_{2} \mathrm{O}\right)$ ) mit orthorhombischer Kristallstruktur (RG: Pnma, $a=11,728(4) \AA, b=11,973(4) \AA$, $c=6,772(2) \AA$; Anderson et al., 2005). 
- Talk - $\mathrm{Mg}_{3} \mathrm{Si}_{4} \mathrm{O}_{10}(\mathrm{OH})_{2}$ - kann entweder in trikliner (Talk-1A, $R G: C \overline{1}, a=5,290(3) \AA$, $b=9,173(5) \AA, c=9,460(5) \AA, \alpha=90.46(5)^{\circ}, \beta=98.68(5)^{\circ}, \gamma=90.09(5)^{\circ}$; Perdikatsis \& Burzlaff, 1981) oder monokliner Kristallstruktur vorkommen (Talk-1M, $R G: C 2 / c$, $a=5,26(2) \AA, b=9,10(2) \AA, c=18,81(3) \AA, \alpha=\gamma=90^{\circ}, \beta=100.08^{\circ}$; Gruner, 1934).

- Calciumfluorid $\left(\mathrm{CaF}_{2}\right)$ hat eine kubische Kristallstruktur ( $R G: F m \overline{3} m, a=5,4712(4) \AA$; Zhurova et al., 1996). Allerdings ist es fraglich, ob $\mathrm{CaF}_{2}$ mit einem Gewichtsanteil von $<0,3$ Gew.\% selbst mit hochenergetischer Röntgenstrahlung zu detektieren ist.

\subsection{Indirekte Füllmaterialien und Werkstoffe für Zahnprothesen}

\subsubsection{Prothesenkunststoffe und Kunststoffzähne}

Zu den Prothesenkunststoffen zählen die zahnfleischfarbenen Kunststoffe, aus denen die Basen von herausnehmbaren Total- oder Teilprothesen gemacht werden. Außerdem werden die durchsichtigen Prothesenkunststoffe für Aufbissschienen (zur Vermeidung der Zahnschädigung bei Bruxismus) verwendet. Typische Prothesenkunststoffe bestehen aus ca. $90 \%$ Methylmethacrylaten, $8 \%$ Vernetzer (z.B. Butandioldimethacrylat) und ca. 2\% Additive (Stabilisatoren, Farbpigmente) und enthalten wenig bis keine anorganischen Füllstoffe (Eichner \& Kappert, 1996). Die Aushärtung der Prothesenkunststoffe erfolgt entweder durch Heiß-, Kaltoder Mikrowellenhärtung. Die hier untersuchten Prothesenkunststoffe sind aussschließlich Kaltpolymerisate, die bei geringem Druck und geringer Wärme aushärten (Tab.4; An-

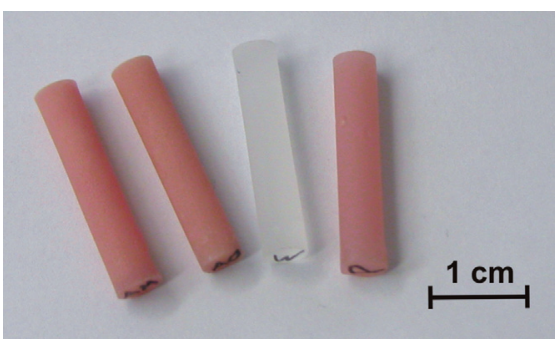

Abb. 18: Prothesenkunststoffe Aesthetic (normaler Faseranteil), Aesthetic (erhöhter Faseranteil), Weitur-Press und PalaPress (von links nach rechts). hang Tab. A.3). Mit Ausnahme des Prothesenkunststoffs Aesthetic sind sie einfarbig (rosa oder durchsichtig); im Fall von Aesthetic werden dem rosa gefärbten Prothesenkunststoff zusätzlich rote Kunststofffasern hinzugefügt.

Neben den oben genannten Prothesenkunststoffen gibt es auch fertige Prothesenzähne aus Kunststoff, deren chemische Zusammensetzung auf Polymethylmethacrylat mit sehr hoher Molmasse (ca. $500.000-1.000 .000 \mathrm{~g} / \mathrm{mol}$ ), weiteren teilweise vernetzten Polymeren, Pigmenten und eventuell hochdispersem Siliziumdioxid basiert, das die Abriebfestigkeit der Kunststoffzähne erhöhen soll (Eichner \& Kappert, 1996). Als Vertreter für fertige Kunststoffzähne wird ein Kunststoffzahn aus der Serie Bioplus der Firma

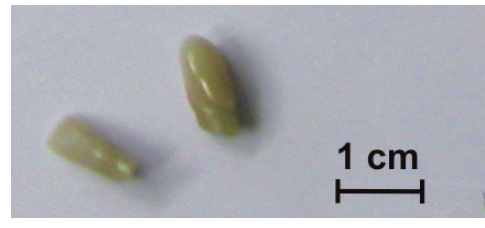

Abb. 19: Prothesenkunststoffzähne aus der Serie Bioplus. Degudent-Degussa untersucht. In diesem Fall wird der INPEN ${ }^{\circledR}{ }_{-K u n s t s t o f f}$ verwendet, der ein sehr dichtes und homogenes Netzwerk aus interpenetrierten Silikon-Copolymeren bildet und frei von anorganischen Füllstoffen ist. Um die natürliche Zahnfärbung und -schattierung optimal wieder zugeben, sind Bioplus-Zähne aus vier (zumindest farblich) unterschiedlichen Schichten aufgebaut. 
Tab. 4: Anwendungsbereiche und Verarbeitung der untersuchten Prothesenkunststoffe.

\begin{tabular}{|c|c|c|c|}
\hline Firma & Handelsname & Indikationen & Verarbeitung \\
\hline $\begin{array}{l}\text { Dentsply- } \\
\text { DeguDent }\end{array}$ & Bioplus & $\begin{array}{l}\text { Kunststoffzahn für die } \\
\text { herausnehmbare Prothese }\end{array}$ & fertig geliefert \\
\hline Weithas & Weitur-Press & $\begin{array}{l}\text { Total-/Teilprothetik, } \\
\text { Modellgussarbeiten, Unterfütterung, } \\
\text { Reparaturen, kieferorthopädische } \\
\text { Apparate }\end{array}$ & $\begin{array}{l}\text { Gießtechnik, Aushärtung } \\
\text { bei } 2 \text { bar und } 45-55^{\circ} \mathrm{C} \text { in } \\
10 \text { Min. }\end{array}$ \\
\hline Kulzer & Pala-Press & $\begin{array}{l}\text { Randgestaltung und Unterfütterung } \\
\text { von Prothesen, Komplettierung von } \\
\text { Modellgußprothesen }\end{array}$ & $\begin{array}{l}\text { Gießtechnik, Aushärtung } \\
\text { bei } 2 \text { bar und } 45-55^{\circ} \mathrm{C} \text { in } \\
10 \text { Min. }\end{array}$ \\
\hline Candulor & $\begin{array}{l}\text { Aesthetic } \\
\text { (normaler } \\
\text { Faseranteil) }\end{array}$ & $\begin{array}{l}\text { Total-/Teilprothetik, } \\
\text { Kombinationsprothetik, } \\
\text { Implantatprothetik, Reparaturen }\end{array}$ & $\begin{array}{l}\text { Gieß- oder Stopftechnik, } \\
\text { Aushärtung bei } 2 \text { bar und } \\
45-55^{\circ} \mathrm{C} \text { in } 10 \text { Min. }\end{array}$ \\
\hline Candulor & $\begin{array}{l}\text { Aesthetic } \\
\text { (erhöhter } \\
\text { Faseranteil) }\end{array}$ & $\begin{array}{l}\text { Total-/Teilprothetik, } \\
\text { Kombinationsprothetik, } \\
\text { Implantatprothetik, Reparaturen }\end{array}$ & $\begin{array}{l}\text { Gieß- oder Stopftechnik, } \\
\text { Aushärtung bei } 2 \text { bar und } \\
45-55^{\circ} \mathrm{C} \text { in } 10 \text { Min. }\end{array}$ \\
\hline
\end{tabular}

\section{Mineralogische Zusammensetzung der Prothesenkunststoffe und Kunststoffzähne}

Sowohl in den Prothesenkunststoffen als auch im Kunststoffzahn der Serie Bioplus sind keine vollkristallinen (anorganischen) Bestandteile zu erwarten. Die Prothesenkunststoffe bestehen hauptsächlich aus Polymethylmetacrylat (PMMA), einem thermoplastischem Kunststoff. Thermoplaste sind hochmolukulare, unverknüpfte Fadenmoleküle, die i.d.R. zu statistischen Knäueln angeordnet sind und nur in einigen Fällen (mikro-)kristalline Bereich aufweisen können. Der Thermoplast PMMA gehört zu den amorphen Thermoplasten ohne kristalline Bereiche, wie es anhand von Rayleigh-Brillouin-Streuung nachgewiesen werden konnte (Patterson, 1976). 


\subsubsection{Dentalkeramiken}

Tab. 5: Anwendungsbereiche und Verarbeitung der untersuchten Dentalkeramiken.

\begin{tabular}{|c|c|c|c|c|}
\hline Firma & Handelsname & Anwendung & Verarbeitung & Zustand \\
\hline $\begin{array}{l}\text { Vita } \\
\text { Zahnfabrik }\end{array}$ & $\begin{array}{l}\text { Vitablocs Mark II } \\
\text { (Farben: A3.5 } \\
\text { und EL) }\end{array}$ & $\begin{array}{l}\text { Inlays, Onlays, Veneers, Teilkro- } \\
\text { nen, Einzelkronen im Front- und } \\
\text { Seitenzahnbereich }\end{array}$ & $\begin{array}{l}\text { CAD/CAM- } \\
\text { System }\end{array}$ & $\begin{array}{l}\text { fertig } \\
\text { gesinterter } \\
\text { Block }\end{array}$ \\
\hline $\begin{array}{l}\text { Vita } \\
\text { Zahnfabrik }\end{array}$ & $\begin{array}{l}\text { Vita In-Ceram } \\
\text { Alumina }\end{array}$ & $\begin{array}{l}\text { Gerüste für Einzelkronen und 3- } \\
\text { gliedrige Brücken im Front- und } \\
\text { Seitenzahnbereich }\end{array}$ & $\begin{array}{l}\text { CAD/CAM- } \\
\text { System }\end{array}$ & $\begin{array}{l}\text { Grünling; } \\
\text { La-Glas } \\
\text { infiltriert }\end{array}$ \\
\hline $\begin{array}{l}\text { Vita } \\
\text { Zahnfabrik }\end{array}$ & $\begin{array}{l}\text { Vita In-Ceram } \\
\text { Zirconia }\end{array}$ & $\begin{array}{l}\text { Gerüste für Einzelkronen und 3- } \\
\text { gliedrige Brücken im Front- und } \\
\text { Seitenzahnbereich }\end{array}$ & $\begin{array}{l}\text { CAD/CAM- } \\
\text { System }\end{array}$ & $\begin{array}{l}\text { Grünling; } \\
\text { La-Glas } \\
\text { infiltriert }\end{array}$ \\
\hline $\begin{array}{l}\text { Ivoclar- } \\
\text { Vivadent }\end{array}$ & IPS e.max Press & $\begin{array}{l}\text { Veneer, Teilkrone, Einzelkrone, } \\
\text { 3-gliedrige Brücke im Front- und } \\
\text { Seitenzahnbereich }\end{array}$ & $\begin{array}{l}\text { Heißpressen } \\
\text { in Form }\end{array}$ & $\begin{array}{l}\text { vor und } \\
\text { nach dem } \\
\text { Heißpressen }\end{array}$ \\
\hline $\begin{array}{l}\text { Ivoclar- } \\
\text { Vivadent }\end{array}$ & $I P S$ e.max $C A D$ & Veneer, Teilkrone, Einzelkrone & $\begin{array}{l}\text { CAD/CAM- } \\
\text { System }\end{array}$ & $\begin{array}{l}\text { fertig } \\
\text { gesinterter } \\
\text { Block }\end{array}$ \\
\hline DeguDent & Cercon base & $\begin{array}{l}\text { Einzelkrone, 3- bis 4-gliedrige } \\
\text { Brücken im Front- und Seiten- } \\
\text { zahnbereich }\end{array}$ & $\begin{array}{l}\text { CAD/CAM- } \\
\text { System }\end{array}$ & $\begin{array}{l}\text { Grünling; } \\
\text { gesintert; } \\
\text { verblendet }\end{array}$ \\
\hline
\end{tabular}

Dentalkeramiken basieren je nach Art der Indikation auf Mineralen mit unterschiedlichen physikalischen Eigenschaften, um die erforderliche Festigkeit und/oder Ästhetik zu erzielen. Ursprünglich wurden leucithaltige Silikat-Glaskeramiken in der Zahnmedizin eingesetzt (vgl. Kap. 1.2.3), wobei sich diese wegen ihrer geringen Festigkeit nicht als Grundgerüst für Kronen oder Brücken eignen. Diese konventionellen Dentalkeramiken werden heute i.d.R. für Verblendungen von Metall- oder Keramikgerüsten verwendet. In dieser Arbeit liegt der Fokus dagegen nicht auf diesen Verblendkeramiken, sondern auf verschiedenen Gerüstkeramiken für Vollkeramik-Systeme (Tab. 5).

\section{Vitablocs Mark II}

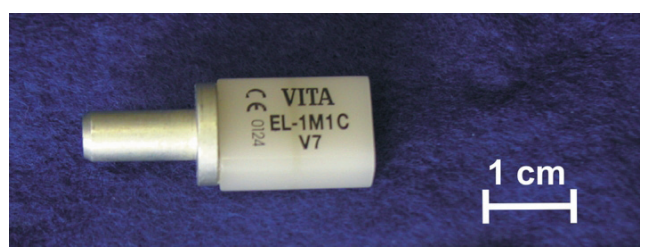

Abb. 20: CAD/CAM-Block der Feldspat-Keramik Vitablocs - Esthetic Line.
Von der chemischen Zusammensetzung her kommt die Dentalkeramik Vitablocs Mark II der Firma Vita Zahnfabrik den konventionellen Dentalkeramiken am nächsten (Abb. 20). Sie werden aus natürlichen Feldspäten unter Zusatz von Farbfritten hergestellt. Nach mehrmaligem Brennen und Mahlen wird das Keramikpulver mit Wasser und Plastifizierungsmittel flieffähig gemacht und über eine Zufuhrschnecke im Schneckenextruder stranggepresst. Diese Keramikstränge werden mehrere

Tage lang in einem klimatisierten Raum getrocknet, dann zersägt, entgratet und auf Brenn- 
trägern im Vakuumofen bei $>1000{ }^{\circ} \mathrm{C}$ gebrannt (Besten et al., 2007). Die fertigen Keramikblöcke können im Dentallabor vor Ort im CAD/CAM-Verfahren (vgl. Kap. 1.2.3) bearbeitet werden, wobei eine Verblendung dieses Keramikgerüstes nicht nötig ist. Von dieser Dentalkeramik werden die Modelle A3.5 und Esthetic Line (EL) untersucht, die sich in der Wahl der Pigmente und in ihrem Glasgehalt unterscheiden.

\section{Vita In-Ceram Alumina und Vita In-Ceram Zirkonia}
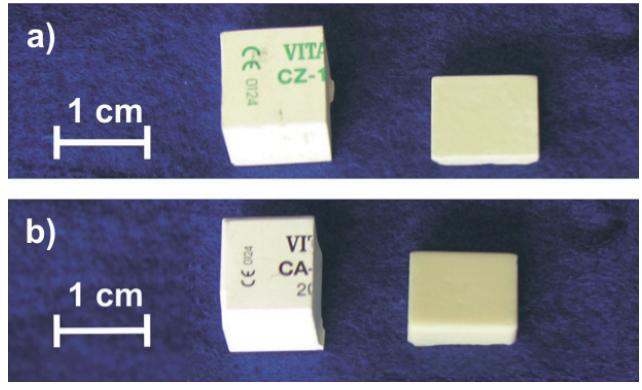

Abb. 21: Vorgesinterte CAD/CAM-Blöcke (links) und La-Glasinfiltrierte Scheiben (rechts) der Keramiken Vita In-Ceram Zirkonia (a) und Vita In-Ceram Alumina (b).

Vita VM.7 bei $900^{\circ} \mathrm{C}$ verblendet.

Das Besondere der Keramik Vita In-Ceram Zirkonia gegenüber Vita In-Ceram Alumina ist, dass das Grundgerüst der Keramik nicht nur aus Korund, sondern auch zu 1/3 aus Zirkoniumdioxid besteht, das durch $12 \mathrm{Mol} \% \mathrm{CeO}_{2}$ in der tetragonalen Hochtemperaturmodifikation bei Raumtemperatur stabilisiert wird. Entstehende Risse in der Keramik werden nicht nur an den Phasengrenzen (Glas-Kristallite) gestoppt, sondern auch durch martensitische Phasenumwandlungen der tetragonalen $\mathrm{Ce}-\mathrm{ZrO}_{2}$ - in eine monokline $\mathrm{Ce}-\mathrm{ZrO}_{2}$-Phase. Die Phasenumwandlung findet bevorzugt unter mechanischen Stresseinwirkungen wie Zugspannung statt, wobei mit der Phasenumwandlung zugleich eine Volumenvergrößerung von 3-5 Gew.\% einhergeht (Nono, 2005; Piconi \& Maccauro, 1999).

\section{IPS e.max Press}

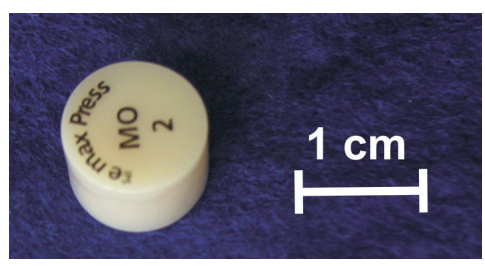

Abb. 22: Press-Rohling der IPS e.max Press. Disilikatkeramik

Als Stellvertreter für die Press-Technik wird in dieser Arbeit IPS e.max Press von der Firma Ivoclar Vivadent untersucht (Abb. 22). Es handelt sich dabei um eine transluszente, teilkristalline Lithiumdisilikat-Glaskeramik (kurz: LS2-Keramik), die auf ca. $920{ }^{\circ} \mathrm{C}$ erhitzt in die Modellform (Einbettmasse IPS Press$V E S T)$ gepresst wird. Farbpigmente werden nicht benötigt, vielmehr wird die Farbe durch Zugabe von farbgebenden Metallionen erreicht, die Farbzentren in der Keramik bilden (Bürke, 2006). Auch die nötige Opazität wird allein durch das langsame Abkühlen der Keramik in der Form erreicht, wobei Lithiumdisilikat $\left(\mathrm{Li}_{2} \mathrm{Si}_{2} \mathrm{O}_{5}\right)$ und Lithium-Metasilikat 
$\left(\mathrm{Li}_{2} \mathrm{SiO}_{3}\right)$ ausscheiden (Bürke, 2006). Nach dem Modellpressen erfolgt abschließend ein Glasurbrand und keine Keramikverblendung.

\section{IPS e.max CAD}

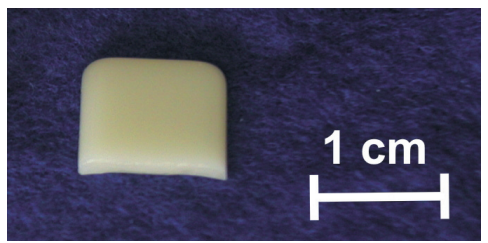

Abb. 23: Fertig gesinterte Scheibe eines $\mathrm{CAD} / \mathrm{CAM}-\mathrm{Blockes}$ von IPS e.max $C A D$.

Nach dem erfolgreichen Einsatz von IPS e.max Press entwickelte Ivoclar Vivadent auch eine Lithiumdisilikat-Keramik mit leicht veränderter chemischer Zusammensetzung für das CAD/CAM-Verfahren: IPS e.max CAD (Abb. 23). Für das $\mathrm{CAD} / \mathrm{CAM}$-Verfahren sollte dabei jedoch nicht eine fertig gesinterte $\mathrm{Li}_{2} \mathrm{Si}_{2} \mathrm{O}_{5}$-Glaskeramik verwendet werden, da die $\mathrm{Li}_{2} \mathrm{Si}_{2} \mathrm{O}_{5}-$ Kristallite der Glaskeramik eine zu hohe Härte verleihen, als dass man sie günstig im CAD/CAM-Verfahren bearbeiten könnte. Daher wird die Kristallisation in zwei Schritten durchgeführt (Bürke, 2006): zunächst wird die Temperatur so eingestellt, dass sich sowohl $\mathrm{Li}_{2} \mathrm{Si}_{2} \mathrm{O}_{5^{-}}$als auch $\mathrm{Li}_{2} \mathrm{SiO}_{3}$-Keime bilden, und dann so weit erhöht, dass nur die $\mathrm{Li}_{2} \mathrm{SiO}_{3}$-Keime wachsen. In diesem Stadium ist die durch Farbzentren blau gefärbte Glaskeramik gut maschinell bearbeitbar und kann (nach dem Abkühlen) in ihre Form gefräst werden. Nach der Formgebung wird die Glaskeramik soweit erhitzt, dass sich die $\mathrm{Li}_{2} \mathrm{SiO}_{3}$-Kristalle auflösen und die $\mathrm{Li}_{2} \mathrm{Si}_{2} \mathrm{O}_{5}$-Keime wachsen $\left(840{ }^{\circ} \mathrm{C}\right)$. Dabei verliert die Keramik ihre blaue Farbe und die $\mathrm{Li}_{2} \mathrm{Si}_{2} \mathrm{O}_{5}$-Kristallite erreichen durch Ostwald-Reifung ihre gewünschte Endgröße. Durch diesen 2-Stufen-Prozess wird eine gute maschinelle Bearbeitbarkeit erreicht, wobei durch die Erstkristallisation von $\mathrm{Li}_{2} \mathrm{SiO}_{3}$ die Glaskeramik im abschließenden Brand bei der Bildung von $\mathrm{Li}_{2} \mathrm{Si}_{2} \mathrm{O}_{5}$ nicht schrumpft (Bürke, 2006).

\section{Cercon base}

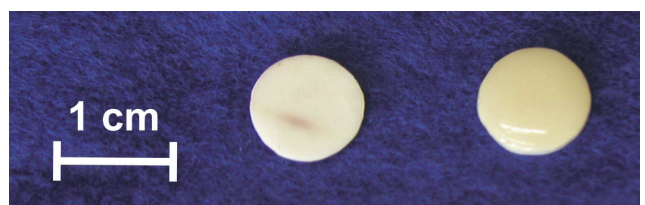

Abb. 24: Fertig gesinterte und verblendete Scheiben der TZPKeramik Cercon base - auf der rechten Seite ist die Verblendkeramik zu sehen.

Bei der Keramik Cercon base (Abb. 24) der Firma DeguDent handelt es sich um eine Zirkoniumdioxid-Keramik, bei der die tetragonale Hochtemperaturmodifikation von $\mathrm{ZrO}_{2}$ durch Zusatz von 5 Gew.\% $\mathrm{Y}_{2} \mathrm{O}_{3}$ bei Raumtemperatur stabilisiert wird. Sie wird im CAD/CAMVerfahren verarbeitet. Da diese tetragonal-stabilisierte Zirkoniumdioxid-Keramik (TZP-Keramik von engl. „tetragonal zirconia polycrystals") im fertig gesinterten Zustand schwer zu bearbeiten ist, wird diese nur vorgesintert - als Grünling - an den Zahntechniker geliefert.

Dort wird sie im CAD/CAM-Verfahren verarbeitet, wobei die Schrumpfung der TZP-Keramik bekannt ist und beim Computer gestützen Fräsen berücksichtigt wird. Zuletzt wird die Keramik fertig gebrannt und mit der Verblendkeramik Cercon Ceram Kiss versehen.

\section{Mineralogische Zusammensetzung der Dentalkeramiken}

Für die Feldspat-Keramiken Vitablocs A3.5 und Esthetic Line wird angegeben, dass sie aus natürlichen Feldspäten hergestellt werden. Das bedeutet, dass aus der Feldspatgruppe zunächst jedes End- und Mischglied als Komponente in Frage kommt. Die Minerale der Feldspatgruppe 
gehören zu den Gerüstsilikaten und sind aus eckenverknüpften $\left[\mathrm{SiO}_{4}\right]$ - bzw. $\left[\mathrm{AlO}_{4}\right]$-Tetraedern aufgebaut, in deren Hohlräumen Kationen eingelagert sind. Die Endglieder der Feldspatgruppe sind - rein chemisch betrachtet - Kalifeldspat $\left(\mathrm{KAlSi}_{3} \mathrm{O}_{8}\right)$, Anorthit $\left(\mathrm{CaAl}_{2} \mathrm{Si}_{2} \mathrm{O}_{8}\right)$ und Albit $\left(\mathrm{NaAlSi}_{3} \mathrm{O}_{8}\right)$. Mischreihen zwischen Orthoklas und Albit werden als Alkifeldspäte und Mischreichen zwischen Albit und Anorthit als Plagioklase bezeichnet. Ihre Kristallklasse und Raumgruppe wird im Wesentlichen durch die Kationen bestimmt: das relativ große $\mathrm{K}^{+}$-Kation braucht mit einem Radius $r=1,60 \AA$ mehr Platz als die kleineren Kationen $\mathrm{Na}^{+}(1,26 \AA)$ und $\mathrm{Ca}^{2+}$ $(1,20 \AA$; Ionenradien für die Koordinationszahl VII nach Shannon (1976)). Das führt dazu, dass K-reiche Feldspäte eher monoklin und Ca- bzw. Na-reiche Feldspäte triklin sind (Okrusch \& Matthes, 2005). Neben den Kationen wird die Kristallstruktur auch durch die Bildungstemperatur bestimmt. Bei hohen Temperaturen sind die Tetraederplätze statistisch von $\mathrm{Si}^{4+}$ - und $\mathrm{Al}^{3+}{ }_{-}$ Kationen besetzt. Bei tieferen Bildungstemperaturen jedoch findet ein Al-Si-Ordnungsvorgang statt, wodurch die Kristallsymmetrie erniedrigt wird (z.B. Kalifeldspat: Sanidin (Hochtemperaturphase, monoklin) $\rightarrow$ Mikroklin (Niedrigtemperaturphase, triklin)). Folgende Endglieder in der Hoch- und Tieftemperaturphase sind möglich:

\section{- K-Feldspäte $\left(\mathrm{KAlSi}_{3} \mathrm{O}_{8}\right)$}

- Tieftemperaturphase Mikroklin mit geordneten $\left[\mathrm{AlO}_{4}\right]-$ und $\left[\mathrm{SiO}_{4}\right]$-Tetraedern $(R G$ : $C \overline{1}, a=8,574(2) \AA, b=12,962(7) \AA, c=7,210(2) \AA, \alpha=90,35^{\circ}, \beta=116.03^{\circ}$, $\gamma=88,8^{\circ}$; Negro et al., 1978)

- Orthoklas mit teilweise geordneten $\left[\mathrm{AlO}_{4}\right]-$ und $\left[\mathrm{SiO}_{4}\right]$-Tetraedern $(R G: C 2 / m$, $a=8,5912(9) \AA, b=13,0009(14) \AA, c=7,1919(7) \AA, \beta=116,009(2)^{\circ}$; Tseng et al., 1995)

- Sanidin mit weitestgehend statistischer $\mathrm{Al}^{3+}{ }_{-} \mathrm{Si}^{4+}{ }_{-}$Verteilung $\left(\mathrm{KAlSi}_{3} \mathrm{O}_{8} ; R G: C 2 / m\right.$, $a=8,603(2) \AA, b=13,036(4) \AA, c=7,174(2) \AA, \beta=116,03(2)^{\circ}$; Ferguson et al., 1991)

\section{- Na-Feldspäte $\left(\mathrm{NaAlSi}_{3} \mathrm{O}_{8}\right)$}

- Tief-Albit mit geordneten $\left[\mathrm{AlO}_{4}\right]$ - und $\left[\mathrm{SiO}_{4}\right]$-Tetraedern $(R G: C \overline{1}, a=8,152(3) \AA$, $b=12,831(4) \AA, c=7,110(2) \AA, \alpha=93,46(2)^{\circ}, \beta=116,52(3)^{\circ}, \gamma=89,72(3)^{\circ}$; Meneghinello et al., 1999)

- Hoch-Albit mit statistisch verteilten $\left[\mathrm{AlO}_{4}\right]$ - und $\left[\mathrm{SiO}_{4}\right]$-Tetraedern, aber trikliner Kristallsymmetrie $(R G: C \overline{1}, a=8,161(1) \AA, \quad b=12,875(2) \AA, c=7,110(1) \AA$, $\alpha=93,53(1)^{\circ}, \beta=116,46(1)^{\circ}, \gamma=90,24(1)^{\circ}$; Winter et al., 1979)

- Hochtemperaturphase Monalbit mit statistisch verteilten $\left[\mathrm{AlO}_{4}\right]-$ und $\left[\mathrm{SiO}_{4}\right]-$ Tetraedern und monokliner Kristallsymmetrie $(R G: C 2 / \mathrm{m}, a=8,274(5) \AA$, $b=12,991(6) \AA, c=7,144(4) \AA, \alpha=\beta=90^{\circ}, \gamma=116,13(4)^{\circ} ; \mathrm{T}_{\text {Messung }}=1253 \mathrm{~K}-$ bei Raumtemperatur instabil; Winter et al., 1979) 


\section{- Ca-Feldspäte $\left(\mathrm{CaAl}_{2} \mathrm{Si}_{2} \mathrm{O}_{8}\right)$}

- Anorthit mit immer vollständig geordneter $\mathrm{Al}^{3+}{ }_{-} \mathrm{Si}^{4+}$-Verteilung; $R G: \quad P \overline{1}$, $a=8,178(2) \AA, b=12,870(3) \AA, c=14,175(3) \AA, \alpha=93,17(2)^{\circ}, \beta=115,97(1)^{\circ}$, $\gamma=91,15(2)^{\circ}$; Angel et al., 1990).

Die Gitterparameter und Winkel der Feldspäte ändern sich in Abhängigkeit von den eingebauten Kationen und von ihren Al-Si-Ordungszuständen (Kroll et al., 1986). Diese Abhängigkeit ist nicht linear und aufgrund der vielen Faktoren ist es sehr schwer, eine unbekannte Feldspatprobe nur anhand der Gitterparameter aus einer Rietveldverfeinerung eindeutig zu bestimmen.

Neben der Feldspatgruppe sind auch die silikatärmeren Feldspatvertreter in den so genannten Feldspat-Keramiken zu erwarten:

- Leucit $\left(\mathrm{KAlSi}_{2} \mathrm{O}_{6}\right)$ : Tiefleucit $\left(R G: I 4_{1} / a, a=13,05476 \AA, c=13,75182 \AA\right.$; Palmer et al., 1997)

- Nephelin (ideale Zusammensetzung: $\mathrm{KNa}_{3}\left[\mathrm{AlSiO}_{4}\right]_{4} ; R G: P 6_{3}$ ): Natürlich vorkommender Nephelin kann z.B. die Zusammensetzung $\mathrm{K}_{1,76} \mathrm{Na}_{5,86} \mathrm{Ca}_{0,13}\left(\mathrm{Al}_{7,84} \mathrm{Fe}_{0,04} \mathrm{Si}_{8,12} \mathrm{O}_{32}\right)$ haben $\left(R G=P 6_{3}, a=9,9979(6) \AA, c=8,3852(11) \AA\right.$; Tait et al., 2003)

In den Keramiken Vita In-Ceram Alumina und Zirkonia kommt vor allem Korund zum Einsatz, der chemisch sehr stabil ist und in trigonaler Kristallstruktur vorliegt ( $R G: R \overline{3} c, a=4,7589(4) \AA$, $c=12,9919(3) \AA ;$ Sawada, 1994).

Die wesentlichen Bestandteile der Lithiumdisilikat-Keramiken IPS e.max Press und CAD sind

- monoklines $\mathrm{Li}_{2} \mathrm{Si}_{2} \mathrm{O}_{5}\left(R G: C c ; a=5,82 \AA, b=14,66 \AA, c=4,79, \beta=90^{\circ}\right.$; Liebau, 1961), das sich pseudo-orthorhombisch verhält ( $R G$ : Ccc2, $a=5,807(2) \AA, b=14,582(7) \AA$, $c=4,773(3) \AA ;$ Jong et al., 1998)

- metastabiles, orthorhombisches $\mathrm{Li}_{2} \mathrm{SiO}_{3}\left(R G: C m c 2_{1}, a=9,396(1) \AA, b=5,396(1) \AA\right.$, $c=4,661(1) \AA ;$ Völlenkle, 1981),

- orthorhombisches $\mathrm{Li}_{3} \mathrm{PO}_{4} \quad(R G:$ Pnma, $a=10,490(3) \AA, \quad b=6,120(2) \AA$, $c=4,9266(7) \AA$; Yakubovich \& Urosova, 1997).

Eine wichtige Rolle spielen die $\mathrm{ZrO}_{2}$-Modifikationen in den Dentalkeramiken Vita In-Ceram Zirkonia und Cercon base. Reines $\mathrm{ZrO}_{2}$ liegt bei Raumtemperatur als monokliner Baddeleyit vor $\left(R G: P 2_{1} / c, a=5,21 \AA, b=5,26 \AA, c=5,37 \AA\right.$; Naray-Szabo, 1936), kann aber durch Zusatz von Lanthanid-Oxiden wie $\mathrm{Y}_{2} \mathrm{O}_{3}$ und $\mathrm{CeO}_{2}$ sowie auch $\mathrm{MgO}$ und $\mathrm{CaO}$ in seiner tetragonalen Hochtemperaturform bei Raumtemperatur stabilisiert werden (Bsp. $\mathrm{Ce}_{0,12} \mathrm{Zr}_{0,88} \mathrm{O}_{2}$ mit RG: $P 4_{2} / n m c, a=3,636(5), c=5,226(3)$; Wang et al., 1999). Der Vorteil dieser tetragonalstabilisierten $\mathrm{Y} / \mathrm{Ce}-\mathrm{ZrO}_{2}$-Keramiken liegt darin, dass sich die tetragonale $\mathrm{Y} / \mathrm{Ce}-\mathrm{ZrO}_{2}-\mathrm{Phase}$ unter mechanischen Einwirkungen (Stress) in die monokline $\mathrm{Y} / \mathrm{Ce}-\mathrm{ZrO}_{2}-\mathrm{Phase}$ umwandelt, wobei zum einen Energie aufgefangen wird und zum anderen der propagierende Riss durch die mit der 
Phasenumwandlung einhergehende Volumenvergrößerung (ca. 3-5 Vol.\% bei Ce-ZrO2, (Nono, 2005); 3 - 4 Vol.\% bei $\mathrm{Y}_{2} \mathrm{O}_{3}-\mathrm{ZrO}_{2}$, (Piconi \& Maccauro, 1999)), ,verheilt" wird. Daher spricht man bei $\mathrm{ZrO}_{2}$-Keramiken auch gerne von selbstheilender Keramik. Allerdings ist die Wirkung der „Selbstheilung“ dadurch beschränkt, dass die tetragonale Phase bei dieser Reaktion aufgebraucht wird. Außerdem muss bei der Synthese von TZP-Keramiken auf die richtige Korngröße geachtet werden, da die Phasenumwandlungsgeschwindigkeit von der Korngröße abhängig ist.

\subsubsection{Metalllegierungen im Dentalbereich}

\section{Goldlegierungen}

Goldlegierungen werden seit Generationen in der Zahnmedizin für Inlays, Onlays sowie Kronen und Brücken eingesetzt. Über Jahre hinweg wurde an der Zusammensetzung dieser Legierungen gearbeitet und heutzutage findet sich eine ganze Palette unterschiedlichster Goldlegierungen auf dem Markt. Hier werden zwei verschiedene Goldlegierungen Degudent $U$ und Degulor $M$ der Firma DeguDent untersucht (Tab. 6). Degudent $U$ ist eine hochgoldhaltige, aufbrennfähige Legierung, d.h. dass sie z.B. für Kronen mit Keramikverblendung geeignet ist. Die Goldlegierung Degulor $M$ dagegen ist nicht aufbrennfähig und wird für Restaurationen ohne Keramikverblendung verwendet.

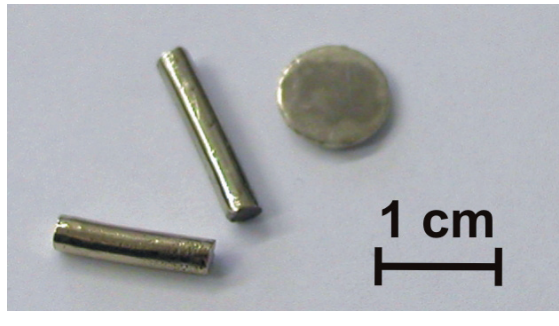

Abb. 25: Die Goldlegierungen Degulor $M$ als Gusskanal (v.l.), Degudent $U$ als Gusskanal (m.) und als Scheibe (r.).

\section{Edelmetallfreie Legierungen}

Neben diesen hochwertigen und relativ teuren Goldlegierungen ist man auch auf der Suche nach günstigeren NEM-Legierungen (Nicht-Edelmetall-Legierungen) wie die hier untersuchte Legierung Wiron 99 (Ni-Cr-Mo-Legierung, Abb. 26), die für Kronen- und Brückengerüste mit Keramikverblendung geeignet ist. Für Modellgussprothesen dagegen eignen sich Co-Cr-Mo-Legierungen wie Remanium GM 800+, die sehr stabil und wenig korrosionsanfällig sind (Abb. 26).

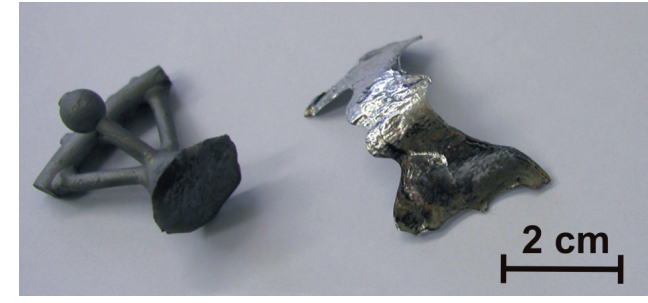

Abb. 26: Gusskanäle von Wiron 99 und fertiges Prothesengerüst Remanium GM $800+$.

\section{Reinmetalle}

Gerade für Implantate und Wurzelstifte werden mechanisch stabile und biologisch gut verträgliche Materialien gesucht. Als Klassiker kommt dabei (kommerziell reines) Titan zum Einsatz, da sich auf dem Titan an Luft sofort eine chemisch inerte, biologisch gut verträgliche Oxidschicht bildet. Als Beispiele wurden hier das Implantat OsseoSpeed der Firma Astra und der Wurzelstift ER Kopfstift untersucht (Abb. 27, Tab. 6). Das Ti-Implantat Osseospeed ist innen hohl und enthält ein Schraubgewinde, während der Wurzelstift aus massivem, kommerziell reinem Titan 
besteht. Kommerziell reines Titan besteht mindestens zu 99 Gew.\% aus reinem Ti und darf in bestimmten Mengen andere Elemente wie N, C, H, Fe und O enthalten. Beide Ti-Dentalwerkstoffe sind medizinisch rein (ASTM-F67: Unlegiertes Titan der Reinheitsgrade 1-4), wobei der Reinheitsgrad des Implantates Osseospeed mit ASTM-Grad 4 näher spezifiziert wird (Dentalkompakt, 2013). Außerdem ist bekannt, dass die Oberfläche des Ti-Implantates mit Titandioxid-Partikeln gestrahlt und fluoridmodifiziert wurde (Dentalkompakt, 2013).
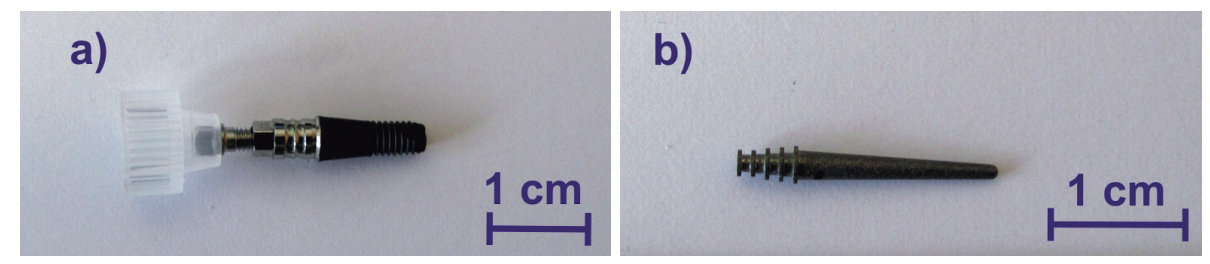

Abb. 27: Titan in der Zahnmedizin: (a) Implantatsystem OsseoSpeed der Firma Astra und (b) Wurzelstift ER Kopfstift der Firma Komet-Brasseler.

\section{Mineralogische Zusammensetzung der Dentallegierungen Edelmetalllegierungen}

Die Kristallstrukturen der reinen Edelmetalle Gold, Palladium, Platin und Silber sind kubischflächenzentriert $\left(F m \overline{3} m ; a_{A u}=4,0782 \AA, a_{P d}=3,8903 \AA, a_{P t}=3,9236 \AA\right.$ und $a_{A g}=408.57 \AA$ (Massalski, 1990)). Diese Edelmetalle sind zu einem großen Teil ineinander löslich und bilden nur wenige Überstrukturen (Bsp. $\mathrm{AuPd}_{3}$, Matsuo (1966)) oder stöchiometrische Verbindungen (Bsp. $\mathrm{Ag}_{15} \mathrm{Pt}_{17}$, Durussel \& Feschotte (1996)). Zusätzlich wurden den Edelmetalllegierungen einige Nichtedelmetalle hinzulegiert, die mit den Hauptmetallen Ausscheidungen bilden, wobei die Anzahl der möglichen Ausscheidungen erheblich größer ist und daher eine Untersuchung der Proben mit der Elektronenstrahlmikrosonde zur näheren Bestimmung nötig ist.

\section{Nichtedelmetalllegierungen}

Im Fall der NEM-Legierungen werden hier zunächst die Kristallstrukturen der reinen Metalle genannt, wobei zu berücksichtigen ist, dass - wie bei den EM-Legierungen - viele stöchiometrische Ausscheidungsphasen möglich sind.

Reines Cobalt und Co-Legierungen mit einem Chrom-Gehalt bis zu maximal 43 Gew.\% haben bei Temperaturen über mindestens $700^{\circ} \mathrm{C}$ eine kubischflächenzentrierte Struktur ( $\gamma$-Co, $F m \overline{3} m$; Gupta (2005)), die bei rascher Abkühlung auch bei Raumtemperatur erhalten bleiben kann. Bei langsamer Abkühlung erfolgt bei CoCr-Legierungen mit einem Cr-Gehalt von 13-35 Gew\% eine Phasenumwandlung in die hexagonal dichteste Kugelpackung ( $\epsilon$-Co, P6 $3 / m m c$; Gupta (2005)). Reines Chrom sowie reines Molybdän liegen in kubisch innenzentrierter Kristallstruktur vor $\left(\operatorname{Im} \overline{3} m, a_{C r}=2,88494(13) \AA\right.$; Straumanis \& Weng (1955) bzw. $a_{M o}=3,1472 \AA$; Swanson \& Tatge (1953)). Reines Nickel ist kubisch flächenzentriert $(F m \overline{3} m, a=3,52394(8) \AA$; Jette \& Foote (1935)). Neben diesen Endphasen gibt es z.B. eine ternäre stöchiometrische Co-Cr-MoVerbindung: $\mathrm{Co}_{49} \mathrm{Cr}_{21} \mathrm{Mo}_{30}$ (R-Phase, $R \overline{3}, a=10,903(5) \AA$ und $c=19,342(11) \AA$ (Komura et al., 1960).

Titan liegt bei Raumtemperatur in hexagonal dichtester Kugelpackung vor $\left(P 6_{3} / m m c, a=\right.$ $2,95111(6) \AA$ und $c=4,68433(10) \AA$ (Wood, 1962)). 
Tab. 6: Anwendungsbereiche und Verarbeitung der untersuchten Edelmetall- und NEMLegierungen.

\begin{tabular}{|c|c|c|c|c|}
\hline Firma & Handelsname & $\begin{array}{l}\text { Chemische Zusammensetzung } \\
\text { [Gew.\%] }\end{array}$ & Anwendung & Verarbeitung \\
\hline DeguDent & Degudent $U$ & $\begin{array}{l}77,3 \mathrm{Au}, 9,8 \mathrm{Pt}, 8,9 \mathrm{Pd}, 1,5 \mathrm{In}, \\
1,2 \mathrm{Ag}, 0,5 \mathrm{Sn}, 0,3 \mathrm{Cu}, 0,2 \mathrm{Re} \\
0,2 \mathrm{Fe}, 0,1 \mathrm{Ir}\end{array}$ & $\begin{array}{l}\text { Einzel- und } \\
\text { Doppelkronen, } \\
\text { Brücken }\end{array}$ & $\begin{array}{l}\text { Guss mit } \\
\text { Keramik- } \\
\text { verblendung }\end{array}$ \\
\hline DeguDent & Degulor $M$ & $\begin{array}{l}70,0 \mathrm{Au}, 4,4 \mathrm{Pt}, 13,5 \mathrm{Ag} \\
8,8 \mathrm{Cu}, 1,2 \mathrm{Zn}, 0,1 \mathrm{Ir}\end{array}$ & $\begin{array}{l}\text { Einzelkronen, } \\
\text { Brücken }\end{array}$ & $\begin{array}{l}\text { Guss ohne } \\
\text { Verblendung }\end{array}$ \\
\hline $\begin{array}{l}\text { Brasseler- } \\
\text { Komet }\end{array}$ & Wurzelstift & Ti (kommerziell rein) & Wurzelstift & fertig geliefert \\
\hline ASTRA & OsseoSpeed & Ti (kommerziell rein, Grad IV) & Implantat & fertig geliefert \\
\hline$B E G O$ & Wiron 99 & $\begin{array}{l}65,0 \mathrm{Ni}, 22,5 \mathrm{Cr}, 9,5 \mathrm{Mo} \\
1,0 \mathrm{Nb}, 1,0 \mathrm{Si}, 0,5 \mathrm{Fe}, 0,5 \mathrm{Ce}\end{array}$ & $\begin{array}{l}\text { Gerüst für } \\
\text { Einzelkronen } \\
\text { und 3-gliedrige } \\
\text { Brücken }\end{array}$ & Guss \\
\hline Dentaurum & $\begin{array}{l}\text { Remanium } \\
\text { GM } 800+\end{array}$ & $\begin{array}{l}63,3 \mathrm{Co}, 30,0 \mathrm{Cr}, 5,0 \mathrm{Mo}, 1,0 \mathrm{Si} \\
(<1 \text { Gew.\%: N, Mn, C, W })\end{array}$ & $\begin{array}{l}\text { Modellguss für } \\
\text { Klammerpro- } \\
\text { thesen }\end{array}$ & Guss \\
\hline
\end{tabular}




\subsection{Füllungen in extrahierten Zähnen}

Neben den neuen Zahnersatzmaterialien wurden auch ,alte“ Füllungen in extrahierten Zähnen röntgenographisch untersucht. Die Dickschliffe von restaurierten und extrahierten Zähnen sind in Abb. 28 zu sehen. Bei beiden Zähnen handelt es sich um prämolare Zähne, von denen einer nur mit Amalgam und der andere mit Gold- sowie einer Unterfüllung restauriert wurde.

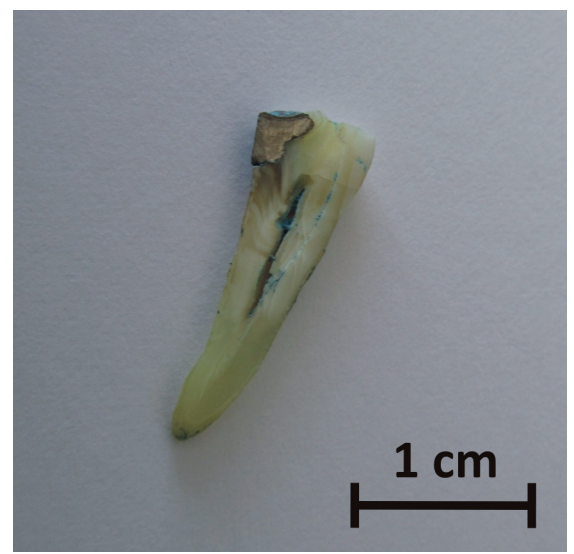

(a) Prämolarer Zahn (24) mit Amalgamfüllung

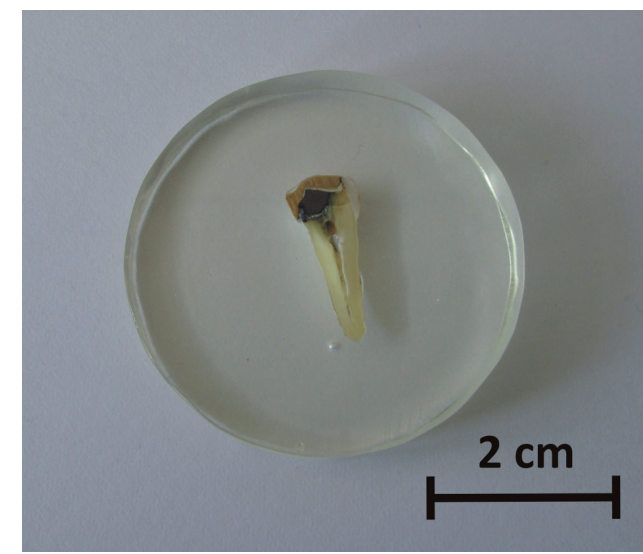

(b) Prämolarer Zahn (15) mit Goldfüllung und Unterfüllung.

Abb. 28: Dickschliffe von extrahierten und eingebetteten Zähnen, die mit verschiedenen Füllmaterialien restauriert wurden. 


\section{Durchführung der Experimente}

\subsection{Herstellung der Proben}

Die Proben wurden vom Universitätsklinikum Göttingen gestellt und in verschiedenen Verarbeitungsgraden untersucht.

\section{Synthese der direkten Füllmaterialien und der Prothesenkunststoffe}

Für die Untersuchungen aller direkten Füllmaterialien wurden in einer Plexiglas-Scheibe zylindrische Löcher mit einer Höhe von $10 \mathrm{~mm}$ und einem Durchmesser von $5 \mathrm{~mm}$ gebohrt (Abb. 29a), in die die Füllmaterialien gestopft wurden. Die Dentalkomposite wurden $20 \mathrm{~s}$ von oben und von unten mit einer UV-Quelle (Bluephase der Firma „Ivoclar“) gehärtet. Die Amalgam-Kapseln Amalcap regular und fast sowie die Applikationskapsel des GIZs „Ketac Fil Plus“ wurden in einen dafür vorgesehenen Schüttler eingespannt, 10 s geschüttelt und anschließend in die zylindrischen Hohlformen einer Plexiglas-Scheibe gestopft, in denen Amalgam und GIZ langsam chemisch aushärteten. Nach dem Füllen der Probenkörper wurden die Proben vorsichtig wieder heraus gelöst.

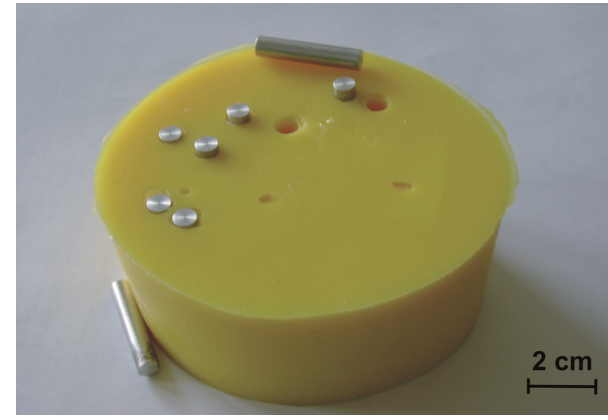

(a) Plexiglas-Scheibe $(10 \times 10 \mathrm{~cm})$ für die Herstellung der Probekörper aus Dentalkompositen, Glasionomerzement und Amalgam.

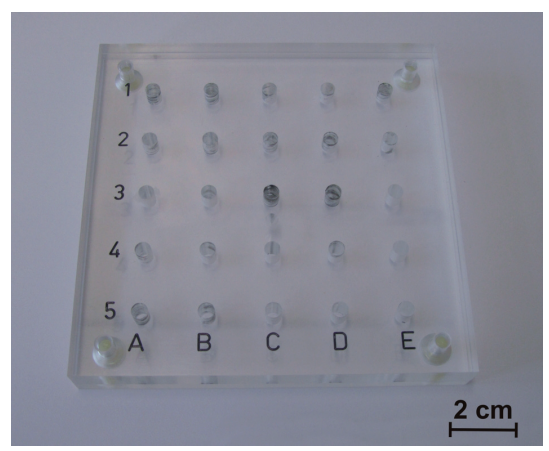

(b) Ausgehärtete Silikonmasse mit den Al-Zylindern (Höhe $=25 \mathrm{~mm}$, Durchmesser $=5 \mathrm{~mm}$ ).

Abb. 29: Syntheseformen für die Herstellung der Probenkörper aus Dentalkompositen, Prothesenkunststoffen, Amalgamen und Glasionomerzementen.

Die Pasten der Zinksulfatzemente Cavit und Detaferm wurden nicht in Hohlformen gestopft, sondern zu wurstigen Gebilden geformt $(\varnothing \approx 6 \mathrm{~mm}$; Abb. 17) und unterschiedlich lange (0 Min., 10 Min., 30 Min. und 3 Tage) in Leitungswasser ausgehärtet.

Für die Herstellung der Proben aus Prothesenkunststoffen wurden kleine Al-Zylinder in SilikonFormmasse Formasil Xact (Abb. 29b) gedrückt und nach dem Festwerden der Silikonmasse durch die Prothesenkunststoff-Masse ersetzt, die in einem Drucktopf bei 2 bar und $45-55{ }^{\circ} \mathrm{C}$ innerhalb von 10 Minuten ausgehärtet wurden.

\section{Synthese der Dentalkeramiken}

Die Dentalkeramikproben wurden in unterschiedlichen Verarbeitungsschritten untersucht. Die 
Keramikblöcke der Feldspat-Keramik Vitablocs Mark II/Esthetic Line wurden z.B. als fertig gesinterte $\mathrm{CAD} / \mathrm{CAM}$-Blöcke geliefert, während andere Keramiken vom Hersteller nur vorgesintert geliefert und erst nach der Formgebung gebrannt werden. Eine Übersicht über die verschiedenen Verarbeitungsgrade ist in Tab. 5 (Kap. 3.2.2) gegeben.

\section{Synthese der Dentallegierungen}

Von den meisten Dentallegierungen konnte Probenmaterial entweder aus Gusskanalresten (Degudent U, Degulor M, Wiron99) oder aus dem fertigen Gussstück (Remanium 800+) entnommen werden. Die Dentallegierung Degudent U wurde zudem mit der Verblendung Duceragold kiss versehen. Die Reintitan-Materialien wie der Wurzelstift und das Implantat Osseospeed wurden im fertigen Zustand geliefert und nicht weiterverarbeitet.

\subsection{Präparation der Proben}

\section{Probenpräparation für die Härtemessungen}

Für die Untersuchung der Proben nach dem instrumentierten Eindringverfahren wurden die Proben in „Technovit 4071“, einem 2-Komponenten-Kunststoff auf Methacrylat-Copolymer-Basis, in runde Formen $(2,4 \mathrm{~cm}$ Innendurchmesser) so eingebettet, dass die jeweilige Probe oben und unten frei liegt. Anschließend wurden die Proben auf einem Schleifband der Firma Buehler mit den Körnungen 240, 320, 400 und 600 Grit geschliffen. Nach jedem Schleifgang wurden die Proben in einem Ultraschallbad für eine Viertelstunde gereinigt. Beispiele der fertigen Proben sind in Abb. 30 zu sehen.

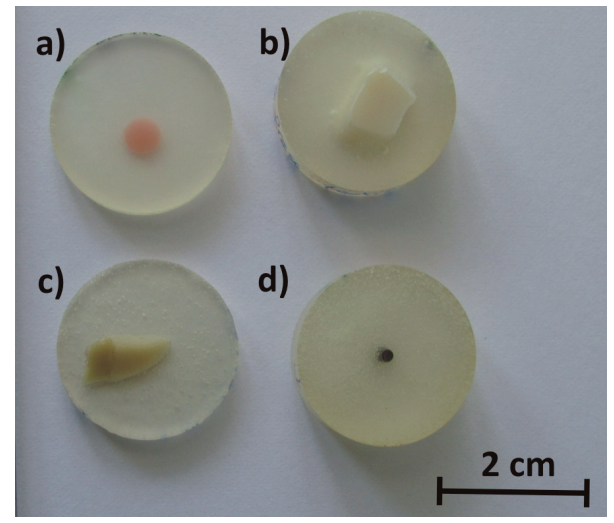

Abb. 30: Proben für die Härtemessungen:

a) Prothesenkunststoff Pala-Press, b) Disilikat-Keramik IPS e.max Press (Block nach Heißpressen), c) Prothesenkunststoffzahn der Serie Bioplus, d) Titan-Wurzelstift.

\section{Aufbereitung der Proben für REM- und Mikrosondenanalysen}

Die Probenpräparation für die REM-Analysen wurde durch die Schleifwerkstatt des GZGs vorgenommen. Von den Keramiken Vitablocs Mark II - Esthetic Line und IPS e.max Press (Rohling) sowie der Goldlegierung Degudent $U$ wurden Dünnschliffe $(30 \mu \mathrm{m})$ und von der Dentallegierung Remanium GM 800+ ein Dickschliff $(400 \mu \mathrm{m})$ erstellt. Die Proben wurden anschließend poliert 
und mit einer dünnen Kohlenstoffschicht bedampft, um den durch den Elektronenstrahl auf der Probe entstehenden Stromfluss ableiten zu können.

Für die Untersuchungen an der Mikrosonde wurden diese Proben übernommen, wobei z.T. die Kohlenstoffbeschichtung erneuert werden musste. Die Dentallegierung Wiron 99 wurde ganz neu eingebettet und im Fall der Dentalkeramik Vita In-Ceram Alumina wurde eine für die Härteuntersuchungen eingebettete Probe übernommen und mit Kohlenstoff bedampft.

\section{Aufbereitung der Proben zur Bestimmung des Glasanteils}

Drei verschiedene Testmischungen wurden für die röntgenographischen Methoden zur Ermittlung des Glasanteils aus Quarzwolle (99.6 Gew.\% rein, Alkali- und Erdalkalimetalle < 0,1 Gew.\%; im Achatmörser zu Pulver verarbeitet), Zinkitpulver (99,999 Gew.\% rein) und Calcitpulver (> 99 Gew.\% rein) hergestellt, wobei als interner Standard das Zinkitpulver mit 10 Gew.\% verwendet wurde (Tab. 7).

Tab. 7: Zusammensetzung der Glaspulver-Testmischungen A, B und C (Angaben in Gew.\%).

\begin{tabular}{cccc}
\hline \hline & Glas & Calcit & Zinkit \\
\hline A & 15,00 & 75,00 & 10,00 \\
B & 40,25 & 49,75 & 10,00 \\
B1 & 39,90 & 49,95 & 10,15 \\
C & 74,92 & 15,09 & 9,99 \\
\hline \hline
\end{tabular}

Für die Anwendung der Glasanalysemethoden auf die Dentalkeramiken mussten die Dentalkeramik-Blöcke (Vita In-Ceram Alumina, Vita In-Ceram Zirkonia, IPS e.max Press und Vita Esthetic Line) zunächst pulverisiert werden. Dazu wurden sie zunächst in einer Spindelpresse grob zerkleinert, bevor sie in einem Mörser zu feinem Pulver weiterverarbeitet wurden. Dem Glaskeramikpulver wurde - wie in der Testserie - soviel Zinkit-Pulver hinzugefügt, dass das gesamte Pulver ca. 10 Gew.\% Zinkit enthält. Das fertige Pulver wurde für die röntgenographischen Untersuchungen in kleine Reinst-Aluminium-Röhrchen $\left(\varnothing_{\text {Innen }}=6,7 \mathrm{~mm}\right)$ sowie in röntgendurchlässige Polyimid-Kapillaren gefüllt $\left(\varnothing_{\text {Innen }} \approx 1,7 \mathrm{~mm}\right)$.

\subsection{Auflicht-Mikroskopie}

Im Institut für allgemeine Metallurgie der technischen Universität Clausthal wurden die NEMLegierungen Remanium 800+ und Wiron99 in Technovit eingebettet und anschließend geschliffen und poliert. Zuletzt wurden die Proben mit V2A-Beize geätzt (typische Zusammensetzung einer V2A-Beize: $100 \mathrm{ml}$ destilliertes Wasser, $100 \mathrm{ml}$ konzentrierte Salzsäure, $10 \mathrm{ml}$ konzentrierte Salpetersäure \& ca. 0,3 ml Dr. Vogels-Sparbeize), um Korn- und Subkorngrenzen sowie Ausscheidungsphasen sichtbar zu machen. 


\subsection{Rasterelekronenmikroskop}

Am Rasterelektronenmikroskop FEI Quanta 200 FEG wurden mit Kohlenstoff bedampfte Dünnschliffe der Dentalkeramiken Vitablocs Mark II - Esthetic Line und IPS e.max Press (Rohling) sowie ein Dickschliff der Au-Pd-Pt-Legierung Degudent U untersucht. Für SE-Bilder wurde der Everhart-Thornley-Detektor und für RE-Bilder sowie die semiquantitative EDX-Analyse ein $\mathrm{Si}(\mathrm{Li})$-Halbleiter-Detektor verwendet. Der Arbeitsabstand betrug 10-12 mm und die Anregungsspannung der $\mathrm{LaB}_{6}$-Kathode war $25 \mathrm{kV}$.

\subsection{Elektronenstrahl-Mikrosonde}

Als Elektronenstrahl-Mikrosonde stand eine JEOL JXA 8900 RL zur Verfügung, die zunächst mit einer W-Kathode und später mit einer $\mathrm{LaB}_{6}-$ Kathode ausgerüstet war. Ausgewählte Proben wurden unter folgenden Bedingungen gemessen:

- Degudent U: W-Kathode mit $20 \mathrm{kV}$ und $40 \mathrm{nA}$ (der hohe Stromfluss war nötig, da für WDS-Analysen (Analysen basierend auf einem wellenlängendispersives System (WDS)) viele L-Linien verwendet werden mussten, die eine deutlich geringere Intensität als die K-Linien aufweisen)

- Vitabocs Esthetic Line: W-Kathode mit $15 \mathrm{kV}$ und $15 \mathrm{nA}$

- Remanium GM 800+: W-Kathode mit $20 \mathrm{kV}$ und $20 \mathrm{nA}$

- Vita In-Ceram Alumina: $\mathrm{LaB}_{6}$-Kathode mit $15 \mathrm{kV}$ und $15 \mathrm{nA}$

- Wiron 99: $\mathrm{LaB}_{6}$-Kathode mit $20 \mathrm{kV}$ und $20 \mathrm{nA}$

\subsection{Inhouse-Messungen am Bruker Smart Apex II}

Das Röntgendiffraktometer Bruker Smart Apex II ist mit einer MoK $_{\alpha}$-Röntgenröhre und einem 2d-Detektor (CCD-Kamera) ausgestattet und eignet sich am Besten für Messungen in Transmissionsstellung. Da jedoch alle Dentalmaterialien die $\mathrm{MoK}_{\alpha}$-Strahlung zu einem großen Teil absorbieren, waren keine Transmissionsmessungen der Proben im Ganzen oder in Scheiben (2-5 mm dick) möglich. Dieses traf auch auf die Dentalkomposite zu, da der organischen Kunststoffmatrix Gläser bzw. Minerale mit hoher atomarer Masse zugefügt wurde, um die für Zahnfüllmaterialien geforderte Mindeströntgenabsorbanz zu erreichen. Die Röntgenabsorbanz von Dentalkompositen sollte gemäß der Norm ISO 4049 mindestens der von menschlichem Dentin, wenn nicht Zahnschmelz entsprechen. Typischerweise wird dafür die Röntgenabsorbanz einer $1 \mathrm{~mm}$ dicken Schicht Dentin bzw. Zahnschmelz mit der von Aluminium in $\mathrm{W}-\mathrm{K}_{\alpha}$-Strahlung verglichen, wobei die Röntgenabsorbanz für Dentin der von Aluminium entspricht und die von Zahnschmelz in etwa doppelt so groß ist $(1 \mathrm{~mm}$ dicke Schicht Zahnschmelz absorbiert gleichviel wie eine $2 \mathrm{~mm}$ 
dicke Al-Scheibe; Dukic et al., 2012). Viele der hier vorliegenden Komposite zeigen eine mindestens um den Faktor 2,5 größere Röntgenabsorbanz als eine gleichstarke Al-Schicht (Dukic et al., 2012). Daher waren Messungen mit Mo- $\mathrm{K}_{\alpha}$-Strahlung in Transmission nur möglich, indem Proben pulverisiert (Kreuzsieb-Fachfeile, Stärke 3$)$ und in dünne Glaskapillare $\left(\varnothing_{\text {Innen }} \approx 0,6 \mathrm{~mm}\right)$ gefüllt wurden. Da die Messungen am hochenergetischen Synchrotron DESY viel versprechender waren, wurden zum Vergleich am Bruker Smart Apex II (Detektor-Probe-Abstand: $6 \mathrm{~cm}$ ) nur folgende Dentalmaterialien zusätzlich gemessen:

- Vita In-Ceram Alumina, Scheibe nach La-Glasinfiltration (900s; Rotation um Probenachse; Detektor bei $2 \theta=0^{\circ}$ )

- IPS e.max Press, Gusskanal nach Heißpressen (900s; Rotation um Probenachse; Detektor bei $\left.2 \theta=0^{\circ}\right)$

- Vitablocs - Esthetic Line (1200s; Rotation um Probenachse; Detektor bei $2 \theta=0^{\circ}$ )

- Wiron, Gusskanal (1800s; Rotation um Probenachse; Detektor bei $2 \theta=30^{\circ}$ )

- Remanium, fertiges Gussstück (900s; Rotation um Probenachse; Detektor bei $2 \theta=30^{\circ}$ )

- Degudent U, Gusskanal (2400s; Rotation um Probenachse; Detektor bei $2 \theta=30^{\circ}$ )

\subsection{Konventionelle Texturmessungen}

Die konventionellen Texturmessungen wurden am Texturgoniometer XRD 3000 PTS der Firma Seifert durchgeführt (Abb. 31), das mit einer Co-Röhre ausgestattet ist (Co-K $\mathrm{K}_{\bar{\alpha}}=1,7903 \AA$ ). Vor dem Start einer Texturmessung wurden die genauen Reflexpositionen mit einem 20-Scan bestimmt und geeignete Untergrundpunkte (jeweils einer vor und einer nach dem zu messenden Reflex) ausgesucht. Für die Texturuntersuchungen wurden zwischen 3 und 6 Reflexe je nach Probe (Kristallsymmetrie der texturierten Phase) gewählt, die jeweils von $\phi=0-360^{\circ}\left(\Delta \phi=3,6^{\circ}\right.$ bzw. $\left.5^{\circ}\right)$ und von $\chi=0-70^{\circ}\left(\Delta \chi=5^{\circ}\right)$ mit ständiger Probentranslation zur Verbesserung der Kornstatistik gemessen wurden. Der Winkel $\phi$ entspricht in der Polfigur dem Winkel $\beta$ und der Kippwinkel $\chi$ dem Winkel $\alpha$. Der Winkel $\chi$ bzw. $\alpha$ wird aufgrund der zunehmenden Defokussierung mit steigendem Kippwinkel nur bis $70^{\circ}$ statt bis $90^{\circ}$ gemessen.

In der Regel wurden für die 20-Scans eine Messzeit von $10 \mathrm{~s}$, für die Polfigurmessung $3 \mathrm{~s}$ und für die Untergrundmessungen 5 s pro Messpunkt gewählt. In Ausnahmefällen, v.a. bei hohem Glasanteil oder bei Auftreten von Ausscheidungsphasen, wurde die Messzeit für den 20-Scan auf 15s und die Messzeit für Polfiguren auf $5 \mathrm{~s}$ für eine bessere Zählstatistik erhöht. Das Blendensystem wurde jeweils so eingestellt, dass der Strahl als Strich (ca. 0,1 x $1 \mathrm{~mm}(\mathrm{BxH})$ für 20-Scan und $1 \times 0,1 \mathrm{~mm}$ für Polfigurmessung) auf der Probe abgebildet wird. 


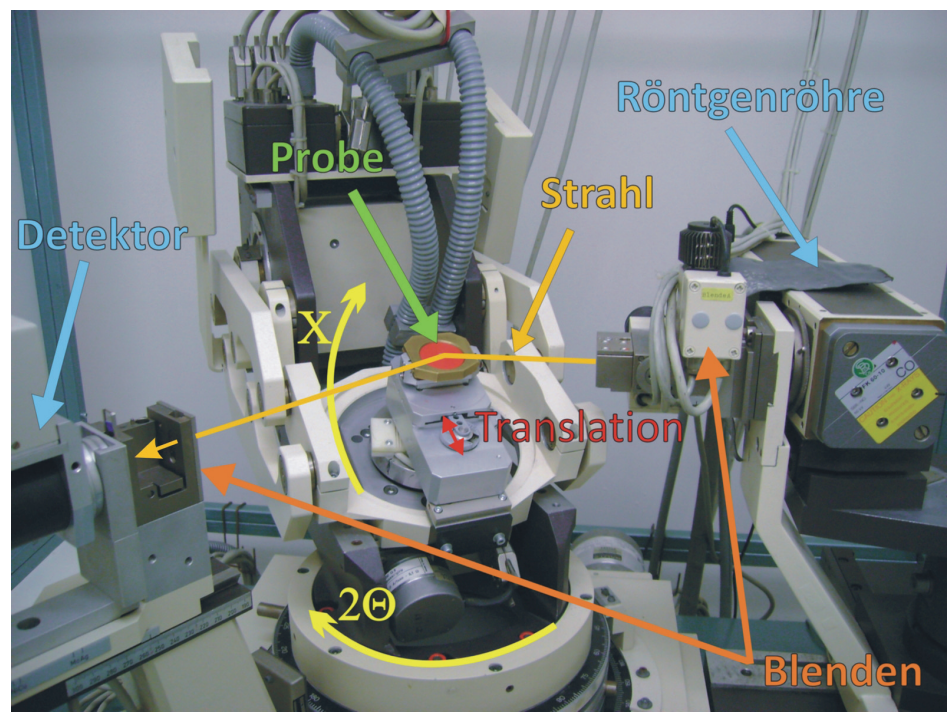

Abb. 31: Texturgoniometer XRD 3000 PTS der Firma Seifert. Auf der rechten Seite ist eine Co-Röntgenröhre zu sehen, die durch einen Punktfokus Co-K ${ }_{\alpha}$-Strahlung in Richtung Probe emittiert. Die Röntgenstrahlung wird durch ein Blendensystem fokussiert, dann an der Probe gebeugt und im Szintillationszähler (links) detektiert. Im Gegensatz zu normalen Pulverdiffraktometern kann an diesem Texturgoniometer neben den Winkeln $\theta$ und $2 \theta$ auch der Kippwinkel $\chi$ eingestellt werden. Zum Erzielen einer besseren Kornstatistik ist zusätzlich eine Translationsbewegung der Probe möglich.

\subsection{Messaufbau am Synchrotron}

Die Synchrotron-Messungen wurden im DESY (Deutsches Synchrotron) in Hamburg durchgeführt. Da die Zahnmaterialien meist eine hohe Röntgenabsorbanz und zudem oft einen hohen Glasanteil aufwiesen, erwies sich die Verwendung von Synchrotronstrahlung wegen der hohen Intensität und des großen Beugungsvolumens als besonders geeignet. Im DESY wurden Röntgenbeugungsexperimente an den Beamlines BW5 (DORIS; HASYLAB), W2 (DORIS; HZG) und P02.1 (PETRA) durchgeführt. Die gewählte Energie betrug meist $\approx 100 \mathrm{keV}$ an den Beamlines BW5 und W2 bzw. $\approx 60 \mathrm{keV}$ an der Beamline P02.1. Die exakte Wellenlänge wurde jeweils vor den Messungen mit Hilfe eines NIST-Korund-Standards bestimmt. Ein typischer Messaufbau für die Beamline BW5 ist in Abb. 32 zu sehen. Für die einfache Phasenanalyse reichte meist ein 2d-Beugungsbild der zu untersuchenden Probe aus. Für die Texturanalyse mit dem kombinierten Textur-Rietveld-Programm MAUD (Material Analysis Using Diffraction; Lutterotti et al. (1997)) hingegen wurden mehrere 2d-Beugungsbilder derselben Probe unter unterschiedlichen Probenwinkeln aufgenommen. Im Idealfall, z.B. bei einer zylindrischen Probe, wurde die Probe von $-90^{\circ} \leq \omega \leq+90^{\circ}$ gedreht und jede $4^{\circ}$ ein Transmissionsbild aufgenommen. Für quaderförmige Proben, bei denen sich die Weglänge des Röntgenstrahls mit zunehmender Drehung vergrößert, wurde nur eine Rotation von $-80^{\circ} \leq \omega \leq+80^{\circ}$ vorgenommen. 


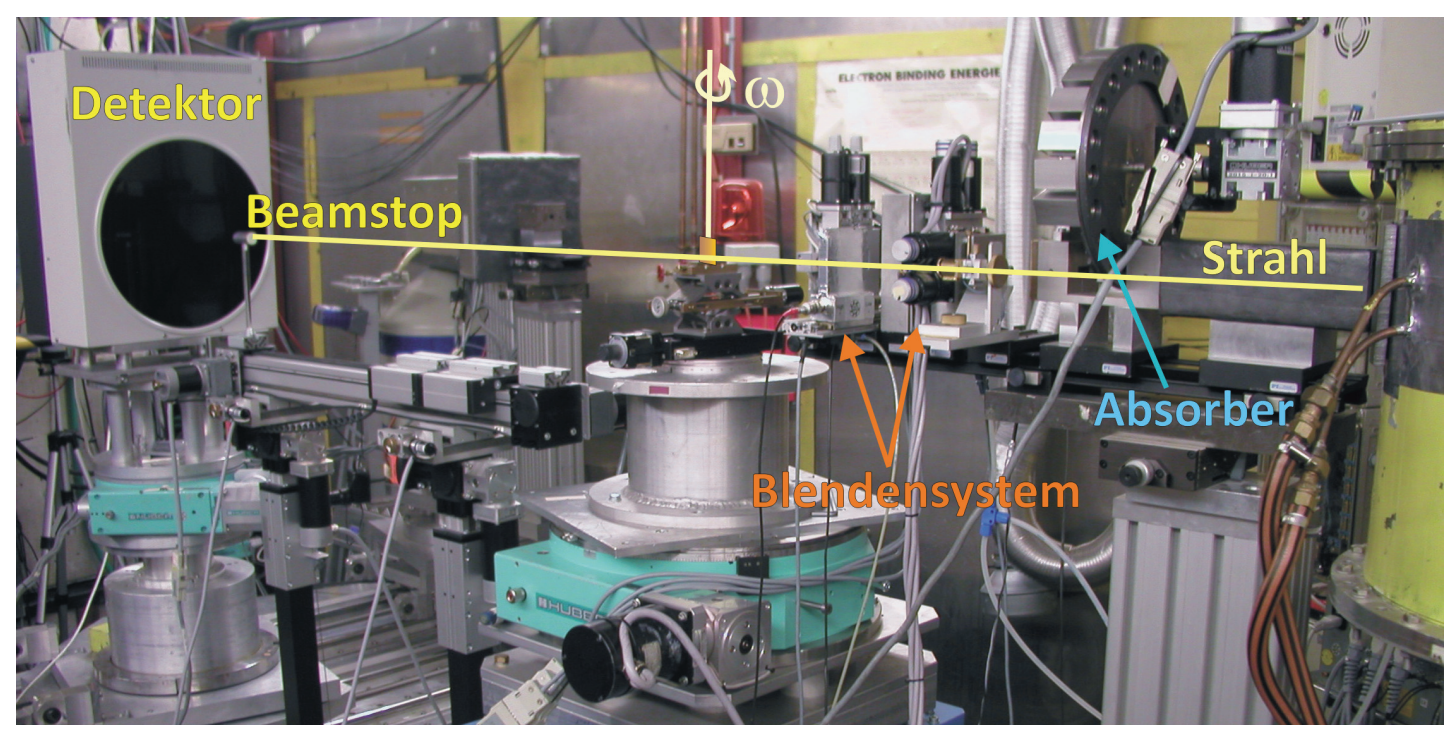

Abb. 32: Synchrotron-Beamline BW5 (HASYLAB, Hamburg). Die gewünschte Wellenlänge, z.B. $0,123 \AA \quad \approx 100 \mathrm{kV})$ wird durch einen Monochromator, meist imperfekter SiGe(111)Kristall, aus dem Synchrotron-Spektrum gefiltert und über Kollimatoren, evtl. Absorber und Blendensystem auf die Probe fokussiert, an der die Strahlung an den Kristallfächen des Probenmaterials im Bragg-Winkel gebeugt wird. Die transmittierte, gebeugte Strahlung wird schließlich mit einem 2d-Detektor erfasst, wobei der Primärstrahl zum Schutz des Detektors durch einen Beamstop absorbiert (hier: mar345 Image Plate Detector, der 2011 durch den schnelleren PerkinElmer-Detektor XRD1621 flat panel detector abgelöst wurde). Für die Texturmessungen wurden die Proben um die Drehachse $\omega$ gedreht.

\subsection{Quantitative röntgenographische Phasen- und Texturanalyse}

Vor Beginn der Phasenanalyse müssen zunächst alle Phasen bekannt sein. Falls eine oder alle Phasen unbekannt sind, kann zur Phasenidentifikation das Programm X'Pert HighScore Plus der Firma PANanalytical verwendet werden. Mit Hilfe dieses Programms werden als erstes der Untergrund und die Reflexpositionen bestimmt. Im nächsten Schritt (,Search \& Match“; Suchkriterien: Position \& Intensität) werden den Reflexen mögliche Phasen zugeordnet, wobei der Anwender vorher festlegen kann, ob organische oder anorganische Phasen vorliegen bzw. welche Elemente in kristallinen Phasen vorkommen. Wenn geeignete Phasen gefunden wurden, kann die eigentliche Rietveld-Analyse beginnen.

Für die Phasenanalyse wurden die Programme GSAS (General Structure Analysis System; Larson \& Van Dreele (1994); Toby (2001)) und MAUD (Material Analysis Using Diffraction; Lutterotti et al. (1997)) verwendet. Das Programm GSAS benötigt einen 20-Scan als Rohdatensatz, während MAUD vollständige 2d-Röntgenbilder als Bilddatei (.tiff; Abb. 33) einlesen kann. Die Kristallstrukturen können in beiden Programmen entweder direkt von einem Strukturdatenfile (.cif) eingelesen oder manuell eingegeben werden. In beiden Programmen stehen neben den Kristallstrukturparametern und Skalierungsfaktoren weitere Parameter wie Untergrundfunktionen, Peakformparameter und Nullpunktsverschiebung für die Phasenanalyse zur Verfügung. Die Möglichkeiten zur Anpassung des Untergrunds unterscheiden sich in beiden Programmen leicht: 
a)

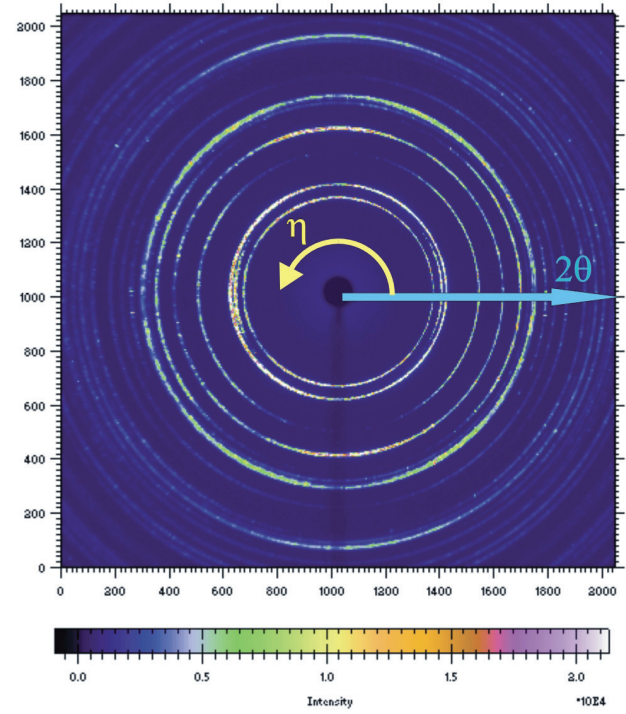

b)

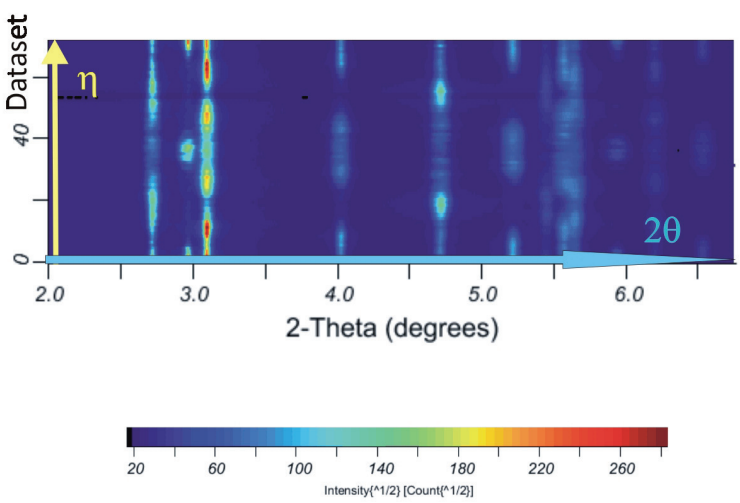

Abb. 33: Datenerfassung mit dem Programm MAUD (Beispiel am Titan-Implantat OsseoSpeed). In (a) ist eine unbearbeitete 2d-Transmissionsaufnahme zu sehen, die für die Rechnung in MAUD in Tortenschnitte (z.B. $\eta$-Winkelbereich von $5^{\circ}$ ) aufgeteilt und zur graphischen Darstellung in ein rechtwinkliges Koordinatensystem übertragen wird (b). Der entsprechende $\eta$-Winkel wird in MAUD im Bild nicht angezeigt, sondern nur die Nummer des Datensatzes für einen $\eta$-Bereich (bei $5^{\circ}$-Schnitten 72 Datensätze).

- GSAS ermöglicht, einen komplizierten Untergrund entweder automatisch oder durch manuell gesetzte Untergrundpunkte polynomial (Tschebyscheff-Polynom) anzupassen. Diese Optionen eignen sich besonders gut bei keinem bis geringem Glasanteil, der den Untergund durch seine amorphe Streuung erhöht.

- MAUD verwendet in der Grundeinstellung frei verfeinerbare Polynomialterme (gut bei einfachem Untergrund), die jedoch durch die Einführung zusätzlicher Gauss-Peaks erweitert werden können. Die zweite Option erleichtert besonders die Untergrundanpassung, wenn ein hoher Glasanteil breite Beugungsringe verursacht.

Neben den genannten Untergrundfunktionen sind weitere in beiden Programmen implementiert, die jedoch für die hier ausgeführten Phasenanalysen keine Bedeutung haben.

Zusätzlich zur normalen Phasenanalyse sind in beiden Programmen Funktionen zur Texturanpassung implementiert, wobei GSAS nicht für eine umfassende Texturanalyse ausgelegt ist. Daher wurden die Texturanalysen mit dem Programm MAUD ausgeführt, mit dem Texturberechnungen nach der E-WIMV-Methode (Lutterotti et al., 2004) - eine Erweiterung der WIMV-Methode (Matthies \& Vinel, 1982) - möglich sind. Details der kombinierten Rietveld-Texturanalyse am Beispiel von Zahnschmelz werden von Raue \& Klein (2011) beschrieben.

Zur Überprüfung der Texturanalyse eignet sich am besten der Vergleich von experimentellen mit berechneten Polfiguren, die aus MAUD über die Funktion „Texture Plot“ als Bild-Datei exportiert werden können. Außerdem kann die ODF als .maa-Datei und die berechneten Polfiguren als .xpc- 
Datei für weitere Analysen mit dem Programm BEARTEX (Berkeley Texture Package; Wenk et al. (1998)) exportiert werden.

Für die Texturanalyse auf Basis konventionell gemessener Polfiguren wurden je nach Kristallsymmetrie der zu untersuchenden kristallinen Phasen die Programme CUBODF (kubische Kristallsymmetrie), HEX-Orth (hexagonale Kristallsymmetrie) und ORTODF (orthorhombische Kristallsymmetrie) verwendet. Diese Programme berechnen die ODF nach der Methode der verallgemeinerten Kugelfunktionen nach Bunge (1993), wobei alle Programme eine orthorhombische Probensymmetrie voraussetzen (Dahlem-Klein et al., 1999).

\subsection{Berechnung der anisotropen physikalischen Eigenschaften eines polykristallinen Werkstoffs mit BEARTEX}

Das Programm BEARTEX (Wenk et al., 1998) enthält Funktionen zur Berechnung von anisotropen physikalischen Eigenschaften eines polykristallinen Materials aus vorhandenen Einkristallund Texturdaten. Dafür müssen zunächst die Texturinformationen aus MAUD importiert werden (2 Möglichkeiten):

- Import der ODF aus MAUD mit der Funktion CMAU erzeugt .YOM-Datei oder

- Import der Polfiguren aus MAUD und Berechnung der ODF nach der WIMV-Methode mit der Funktion WIMV erzeugt.XPX-Datei mit Polfiguren sowie eine .YOD-Datei mit ODF.

Für die Berechnung der makroskopisch anisotropen physikalischen Eigenschaften einer polykristallinen Phase müssen nacheinander die Funktionen TENS und VELO verwendet werden:

- TENS erstellt aus den Einkristalldaten (.tea-Datei mit Eigenschaftstensor, Tensorrang und Mineraldichte) und der Texturinformation (.YOM oder .YOD) einen mit der Textur gewichteten Tensor (.ten-Datei), wobei z.B. die Mittelung für den E-Modul jeweils nach Voigt (1928), Reuss (1929) und Hill (1952) sowie rein geometrisch durchgeführt wird (Ausgabeformat: .ten).

- VELO führt .ten-datei aus und gibt eine .xvx-Datei aus, die den richtungsabhängigen EModul nach Voigt (1928) in GPa zur Darstellung als stereographische Projektion enthält.

Die graphische Darstellung der stereographischen Projektion des E-Moduls erfolgt schließlich mit der Funktion PING in BEARTEX oder mit dem (externen) Programm PFPlot-Ullemeyer (geschrieben von C. Umlauf und K. Ullemeyer 2006; siehe Küster et al., 2010). Im Fall der thermischen Ausdehnung wurde nach der Funktion TENS das (externe) Programm ten2plot v1.4cp (Raue, 2012) anstelle der Funktion VELO verwendet.

Für monokline Kristallsysteme ist jedoch zu beachten, dass in MAUD für Texturrechnungen die c1-Aufstellung (c-Achse als ausgezeichnete Achse; $\alpha=\beta=90^{\circ} \neq \gamma$ ) empfohlen wird. Außerdem braucht auch das Programm BEARTEX zur korrekten Berechnung von Texturen und physikalischen Eigenschaften die c1-Aufstellung. Da jedoch in kristallographischen Arbeiten meist die 
b1-Aufstellung (b-Achse als ausgezeichnete Achse; $\alpha=\gamma=90^{\circ} \neq \beta$ ) verwendet wird, ist eine Achsentransformation nötig: $a_{(b 1)} \rightarrow b_{(c 1)}, b_{(b 1)} \rightarrow c_{(c 1)}$ und $c_{(b 1)} \rightarrow a_{(c 1)}$. Im Fall von symmetrischen Tensoren zweiten Ranges (Bsp.: thermische Ausdehnung) ergibt sich die Transformation nach Gleichung 44.

$$
\left[\begin{array}{ccc}
a_{11}^{\prime} & 0 & a_{13}^{\prime} \\
0 & a_{22}^{\prime} & 0 \\
a_{13}^{\prime} & 0 & a_{33}^{\prime}
\end{array}\right] \stackrel{b 1 \rightarrow c 1}{\longrightarrow}\left[\begin{array}{ccc}
a_{11}=a_{33}^{\prime} & a_{12}=a_{13}^{\prime} & 0 \\
a_{12}=a_{13}^{\prime} & a_{22}=a_{11}^{\prime} & 0 \\
0 & 0 & a_{33}=a_{22}^{\prime}
\end{array}\right]
$$

In dieser Gleichung stehen die $a^{\prime}$-Koeffizienten für die b1-Aufstellung und die $a$-Koeffizienten für die c1-Aufstellung. Die Herleitung der Transformation im monoklinen System für symmetrische Tensoren vierten Grades wird von Matthies \& Wenk (2009) beschrieben und wie folgt durchgeführt:

$$
\left[\begin{array}{ccccccc}
c_{11}=c_{33}^{\prime} & c_{12}=c_{13}^{\prime} & c_{13}=c_{23}^{\prime} & c_{14} *=c_{36}^{\prime} * & c_{15}=c_{34}^{\prime} * & c_{16}=c_{35}^{\prime} \\
& c_{22}=c_{11}^{\prime} & c_{23}=c_{13}^{\prime} & c_{24} *=c_{16}^{\prime} * & c_{25}=c_{14}^{\prime} * & c_{26}=c_{15}^{\prime} \\
& & c_{33}=c_{22}^{\prime} & c_{34}=c_{26}^{\prime} * & c_{35}=c_{24}^{\prime} * & c_{36}=c_{25}^{\prime} \\
& & & c_{44}=c_{66}^{\prime} & c_{45}=c_{46}^{\prime} & c_{46}=c_{56}^{\prime} \\
& & & & c_{55}=c_{44}^{\prime} & c_{56}=c_{45}^{\prime} \\
& & & & & c_{66}=c_{55}^{\prime}
\end{array}\right]
$$

Hierbei entsprechen $c$-Koeffizienten der c1-Aufstellung und $c^{\prime}$-Koeffizienten der b1-Aufstellung. Außerdem sind Koeffizienten, die den Wert 0 annehmen, durch Sternchen $\left({ }^{*}\right)$ markiert und symmetrisch gleichwertige Koeffizienten sind der Übersichtlichkeit wegen nicht dargestellt. ${ }^{1}$

\footnotetext{
${ }^{1}$ Die Eigenschaftstensoren der thermischen Ausdehnung und des E-Moduls sind symmetrische Tensoren, d.h. $a_{i j}=a_{j i}$.
} 


\subsection{Anwendung des instrumentierten Eindringverfahrens zur Ermittlung der Vickershärte und des E-Moduls}

Zur Ermittlung der Vickershärte und des elastischen Eindringmoduls wurde das Mikrohärtemesssystem Fischerscope ${ }^{\circledR}$ H100SMC verwendet (Abb. 34), mit dem Prüflasten zwischen 1-1.000 mN möglich sind. Für die Dentalkomposite sowie die Prothesenkunststoffe wurde eine Prüflast von $100 \mathrm{mN}$ und für die Dentalkeramiken sowie die Dentallegierungen eine Prüflast von $300 \mathrm{mN}$ gewählt, sodass die Eindringtiefen des Indenters bei 1-3 $\mu$ m lagen. Die Aufbringdauer entsprach mit $20 \mathrm{~s}$ der Haltedauer und der Entlastungsdauer.

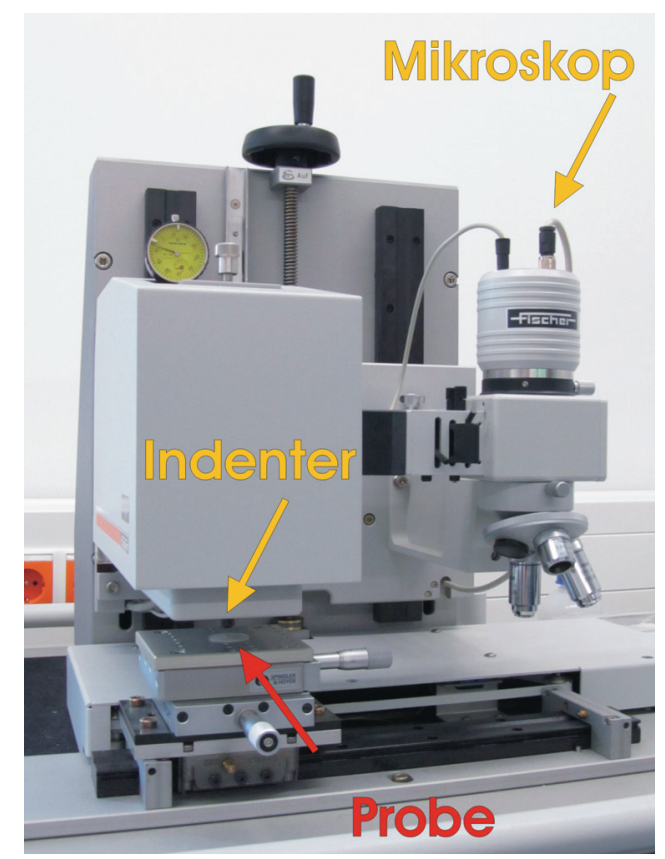

Abb. 34: Mikrohärtemesssystem Fischerscope ${ }^{\circledR}$ H100C der Firma Fischer. 


\section{Ergebnisse}

\subsection{Ergebnisse der Auflichtmikroskopie}

Es wurden Auflichtbilder von den NEM-Legierungen Wiron 99 und Remanium GM 800+ aufgenommen (Abb. 35 und 36). Beide Legierungen wiesen große Korngrößen (>1mm) sowie viele, kleine Porenräume auf. Bei der Ni-Cr-Mo-Legierung Wiron 99 konnte man ein sehr ausgeprägtes dendritisches Wachstum einer Ausscheidungsphase innerhalb der Körner beobachten, wobei sich innerhalb dieser Ausscheidungsphase oft eine weitere Ausscheidungsphase befand (s. Abb. 35c und d). An den Korngrenzen endete meist die dendritische Ausscheidungsphase, sodass direkt in den Korngrenzen kaum Ausscheidungen vorhanden waren (s. Abb. 35d).

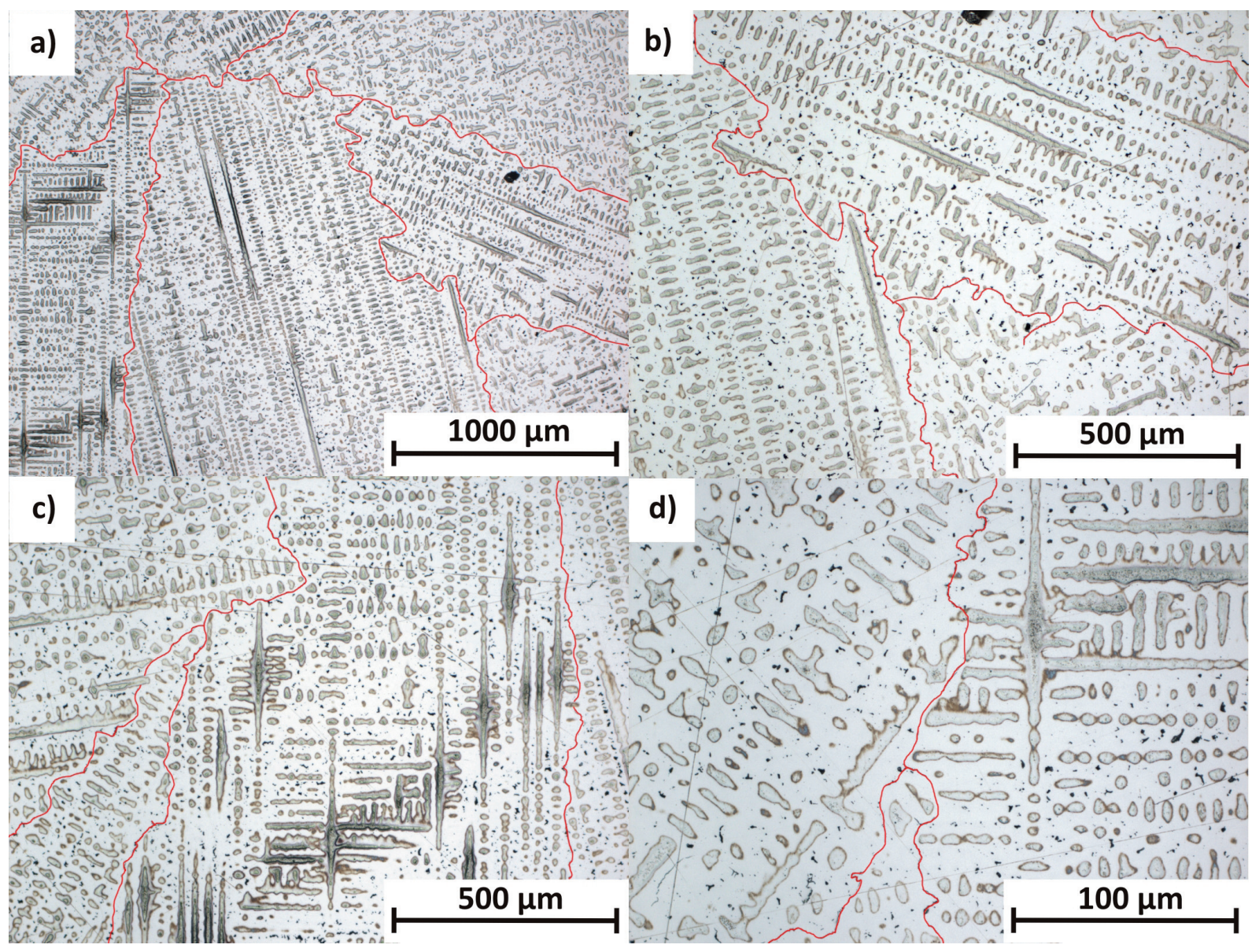

Abb. 35: Auflichtaufnahmen der Ni-Cr-Mo-Legierung Wiron 99. Große Körner (Korngrenzen sind rot nachgezeichnet) sind von dendritischen Ausscheidungen durchzogen (a, b). Die dendritischen Ausscheidungen weisen im Kern oft eine weitere Ausscheidungsphase auf (c, d).

Auch bei der Co-Cr-Mo-Legierungen konnte man eine Ausscheidungsphase sehen, deren Körner sich wie auf einem Gitter anordneten. Im Gegensatz zu Wiron 99 konnte man besonders an den Korngrenzen eine Anhäufung von Ausscheidungen erkennen (Abb. 36e und f). 


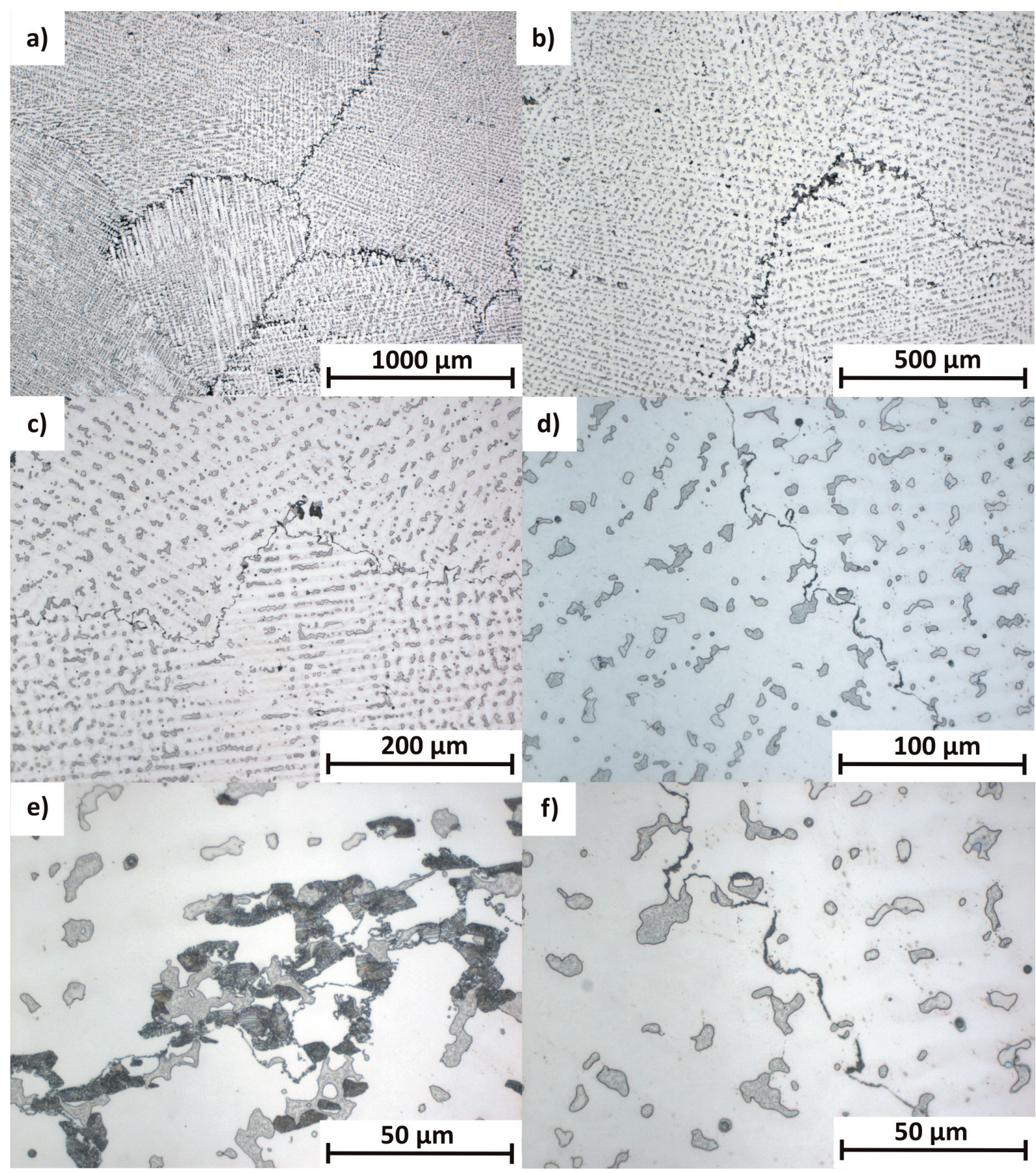

Abb. 36: Auflichtaufnahmen der Co-Cr-Mo-Legierung Remanium GM 800+ in verschiedenen Vergrößerungen. In (a) und (b) sind Ausscheidungen innerhalb großer ( $\geq 1 \mathrm{~mm})$ Körner zu sehen. Diese sind in regelmäßigen Abständen als durchbrochene Linien innerhalb eines Korns angeordnet. (c) und (d) zeigen den Verlauf von Subkorngrenzen. (e) zeigt eine Korngrenze mit zwei verschiedenen Ausscheidunsphasen (erste leicht bräunlich, zweite dunkelgrau gefärbt) und (f) eine fast ausscheidungsfreie Subkorngrenze. 


\subsection{Ergebnisse aus den Untersuchungen am REM}

\section{Vitablocs - Esthetic Line}

Aus den RE-Bildern zusammen mit der standardfreien EDX-Analyse wurde ersichtlich, dass es sich bei den meisten (hell erscheinenden) Körnern um Sanidin-reiche Feldspäte handelte, die in einer Na- und K-reichen Feldspatglasmatrix schwammen (Abb. 37a; Anhang Tab. A.5). Neben diesen Sanidin-Körnern waren auch kleinere, dunklere Körner zu sehen (Abb. 37a und b), die meist einen hellen Saum bzw. Dendritenwachstum aufwiesen (Abb. 37c). Aufgrund der kleinen Kristallitgröße war es nicht immer möglich, reine Analysen von diesen dunkleren Körnern zu erhalten. In zwei Fällen konnten eindeutig Albit-reiche Feldspatkomponenten (Si/Al-Verhältnis: 3:1) nachgewiesen werden und in zwei weiteren Fällen deutet das Si/Al-Verhältnis (2:2) auf eine Nephelinphase hin (s. Tab. A.5).

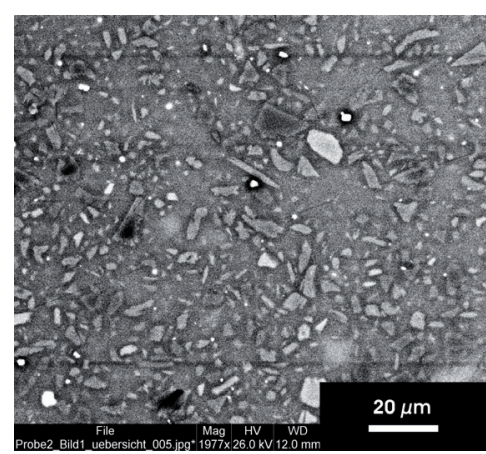

(a) Übersichtsaufnahme.

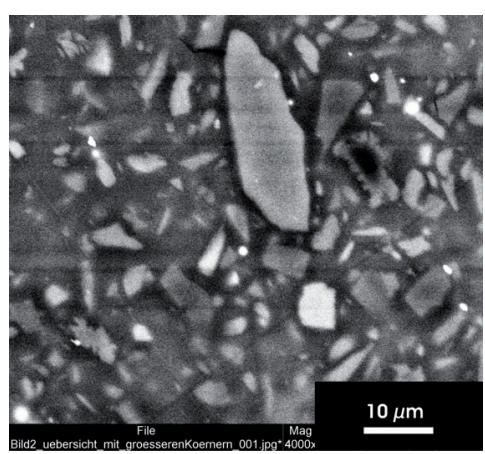

(b) Beispiel für ein etwas größeres Feldspatkorn.

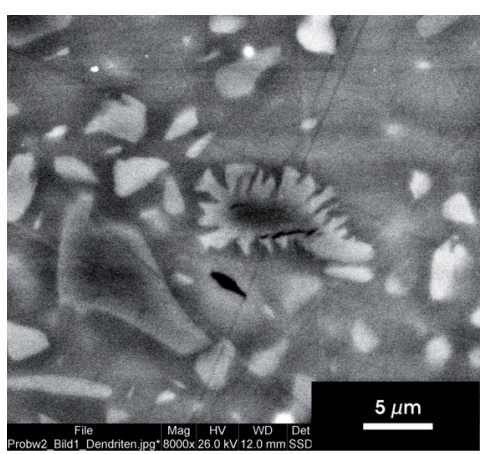

(c) Beispiel für ein Nephelinkorn mit Dendritenwachstum.

Abb. 37: RE-Bilder an einem Dünnschliff der Keramik Vitablocs - EL. Zu sehen sind größtenteils Feldspatkörner in einer Na-K-Al-Si-Glasmatrix. Die relativ schwachen Massenkontraste sind hauptsächlich auf Unterschiede in der Na- und K-Verteilung zurückzuführen.

Die Bestimmung der Korngröße anhand der RE-Bilder war problematisch, da die Korngrößen stark variierten und manche Körner nur bei sehr hoher Auflösung zu sehen waren (Abb. 37c). Die kleinsten Korngrößen wurden um $1 \mu \mathrm{m}$ und die größten um $30 \mu \mathrm{m}$ detektiert.

\section{IPS e.max Press}

Die RE-Bilder der Lithiumdisilikat-Keramik IPS e.max Press zeigten ein sehr homogenes und sehr feinkörniges Gefüge (Abb. 38a). Mit dem EDX-Detektor konnte jedoch aufgrund der kleinen Korngröße $(<1 \mu \mathrm{m})$ nur eine Mischanalyse durchgeführt werden. Zudem konnte das Hauptelement Lithium wegen der zu geringen atomaren Masse mit dem vorhandenen Rasterelektronenmikroskop nicht detektiert werden, wodurch die EDX-Analyse nur Informationen über die Anteile der anderen Elemente lieferte. Neben dem Hauptelement Silizium wurden Zink, Kalium, Phosphor, Natrium und Cer detektiert (s. Anhang Tab. A.6). 


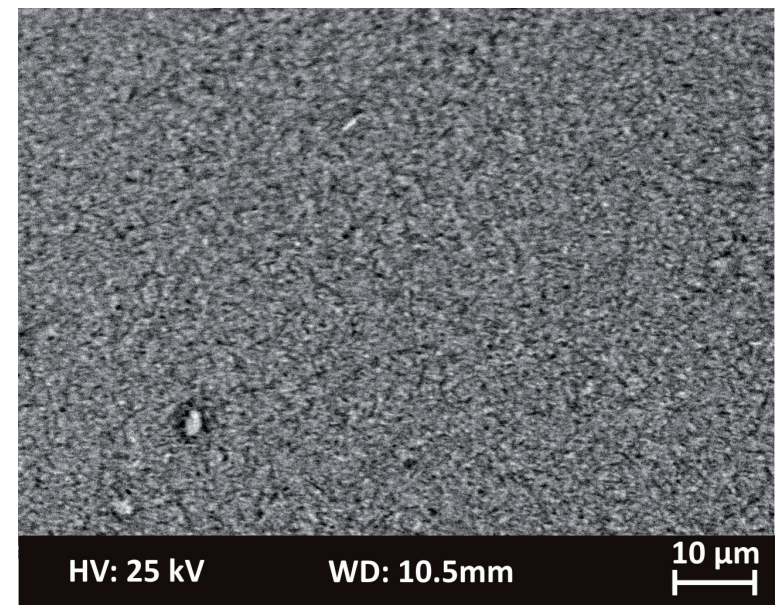

(a) IPS e.max Press im vorgesinterten Zustand: Die Keramik erscheint sehr feinkörnig und ohne starke Massenkontraste. Die Risse rühren von der Präparation des Dünnschliffes her.

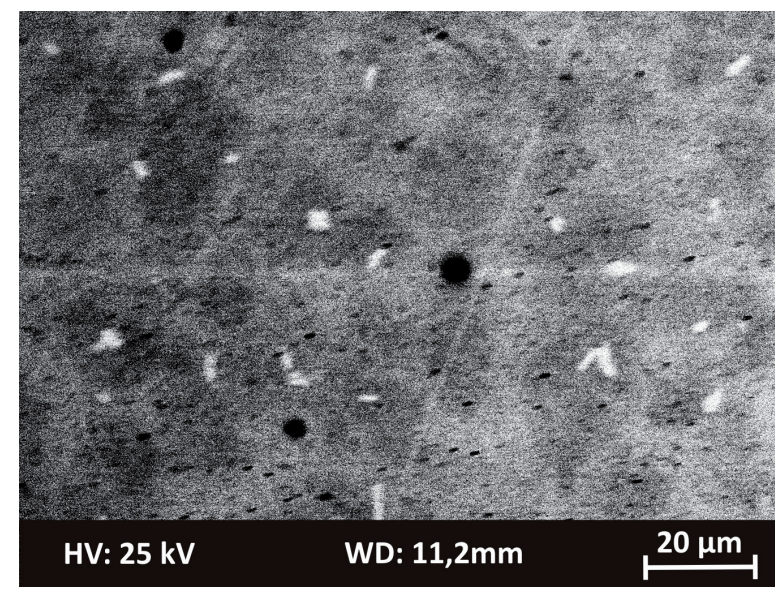

(b) Degudent U: In der Matrix lässt sich eine Entmischung von Au-reichen (hell) zu Pd-reichen (dunkel) Bereichen beobachten, außerdem sind Porenräume und eine Re-haltige, hell erscheinende Ausscheidungsphase zu sehen.

Abb. 38: Rückstreuelektronenbilder von der LS2-Keramik IPS e.max Press und der Goldlegierung Degudent $U$.

\section{Degudent $U$}

Im RE-Bild war eindeutig eine Separation von leichteren und schwereren Elementen innerhalb einer Au-Pt-Pd-Matrix (73-77 Gew.\% Au, 20-22 Gew.\% Pt, 3-6 Gew.\% Pd) zu erkennen, ebenso mindestens eine Re-reiche Ausscheidungsphase (genaue Zusammensetzung unbekannt, da Mischanalysen vorlagen; Abb. 38b). Allerdings konnten mit dem EDX-Detektor nur die Hauptelemente $\mathrm{Au}, \mathrm{Pt}$ und Pd sowie Re bei hoher Anreicherung detektiert werden, da der Anteil der weiteren Legierungskomponenten zu gering war bzw. ihre Röntgenemissionslinien im EDX-Spektrum nicht mehr aufgelöst werden konnten. Genauere chemische Analysen lieferten die Untersuchungen an der Elektronenstrahlmikrosonde mit einem wellenlängendispersiven System. 


\subsection{Ergebnisse aus den Untersuchungen mit der Elektronenstrahlmikrosonde}

\section{Vitablocs Esthetic Line}

Um eine Übersicht über die Probe zu erhalten und die Untersuchungen am REM zu bestätigen, wurden WDS-Analysen (Analysen basierend auf dem wellenlängendispersiven System) an einzelnen Körnern und der Matrix durchgeführt sowie RE-Bilder aufgenommen. In Abb. 39 kann man eine einheitliche Glasmatrix erkennen (gemittelte Zusammensetzung: $\mathrm{Na}_{0,58} \mathrm{Ca}_{0,02} \mathrm{~K}_{0,46} \mathrm{Al}_{1,1} \mathrm{Si}_{2,9} \mathrm{O}_{8}$ ), in der hauptsächlich hell- bis dunkelgrau erscheinende Körner $\left(\mathrm{Na}_{0,52} \mathrm{~K}_{0,56} \mathrm{Al}_{1,1} \mathrm{Si}_{2,9} \mathrm{O}_{8}\right.$, Alkalifeldspat) schwimmen. Als Nebenbestandteile treten im RE-Bild fast schwarz bzw. weiß erscheinende Körner auf. Die weißen Körner konnten mittels qualitativer EDX-Analyse als Zr-Si und Ti-haltige Minerale (vermutlich Zirkon - $\mathrm{ZrSiO}_{4}$ und Rutil - $\mathrm{TiO}_{2}$ ) bestimmt werden, die der Feldspatkeramik wahrscheinlich als Farbpigmente zugesetzt wurden. Die chemische Zusammensetzung der schwarzen Körner deutete auf eine Albit-Komponente hin $\left(\mathrm{Na}_{0,97} \mathrm{~K}_{0,10} \mathrm{Al}_{1,06} \mathrm{Si}_{2,93} \mathrm{O}_{8}\right)$. Allerdings ist zu berücksichtigen, dass hier (wie auch am REM) insgesamt nur wenig Einzelanalysen vorliegen (s. Tab. 8), sodass z.B. die vorher am REM nachgewiesene Nephelinphase bei diesen WDS-Analysen möglicherweise übersehen wurde.

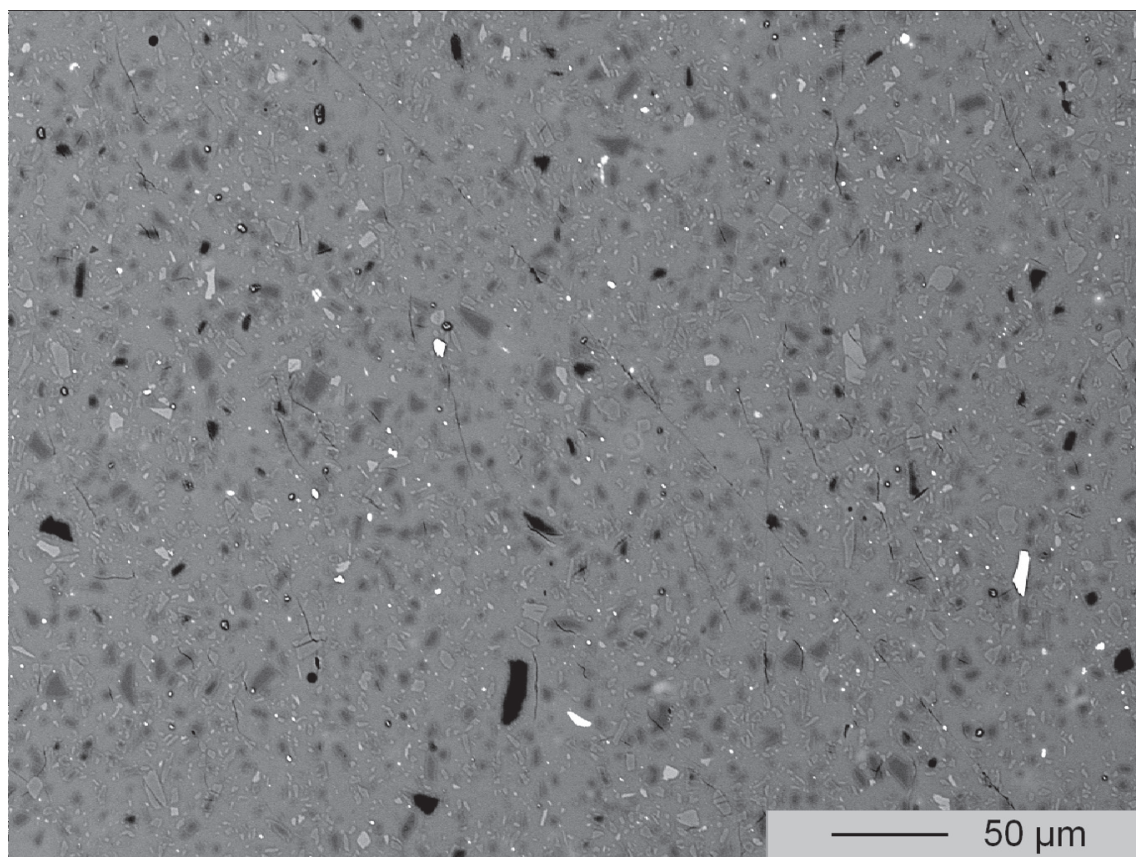

Abb. 39: RE-Übersichtsbild der Keramik Vitablocs - Esthetic Line. In einer hellgrauen Matrix sind hauptsächlich dunkelgraue bis schwarze Körner (Alkalifeldspäte mit variierendem Naund K-Anteil) und vereinzelt sehr helle Körner (wahrscheinlich Farbpigemente wie Rutil und Zirkon) zu sehen.

\section{VITA In-Ceram Alumina}

Die RE-Aufnahme (Massenkontrastbild) der Korund-Keramik VITA In-Ceram Alumina zeigte zwei verschiedene Phasen: rundliche, dunkel erscheinende Korundkörner $\left(d_{\text {mittel }} \approx 1 \mu \mathrm{m}\right)$ und eine helle erscheinende Glasmatrix. Außerdem sind vereinzelt Porenräume in derselben Größe wie die Korundkörner zu sehen (Abb. 40). Die Hauptelemente der Glasmatrix wurden zunächst 
Tab. 8: Chemische Zusammensetzung der Hauptphasen der Dentalkeramik Vitablocs $\boldsymbol{E L}$ aus WDS-Analysen ${ }^{\mathbf{1}}$. Standardabweichungen der Einzelanalysen vom Mittelwert sind in Klammern angegeben.

\begin{tabular}{ccccccccc}
\hline \hline & & $\mathrm{Na}$ & $\mathrm{K}$ & $\mathrm{Ca}$ & $\mathrm{Al}$ & $\mathrm{Si}$ & Summe & $\mathrm{n}^{2}$ \\
\hline \multirow{2}{*}{ Matrix } & Gew.\% & $6(2)$ & $7,71(1)$ & $0,08(1)$ & $20,1(2)$ & $62(1)$ & $96,90(9)$ & 5 \\
& $\mathrm{apfu}^{3}$ & $0,49(15)$ & $0,458(4)$ & $0,02(12)$ & $1,102(1)$ & $2,9(1)$ & $5,1(1)$ & 5 \\
\hline \multirow{2}{*}{ Sanidin } & $\mathrm{Gew} \%^{2}$ & $6(2)$ & $9(3)$ & $0,09(4)$ & $20,1(2)$ & $62(3)$ & $97,7(9)$ & 2 \\
& $\mathrm{apfu}^{3}$ & $0,42(16)$ & $0,56(19)$ & $0,004(3)$ & $1,1(1)$ & $2,90(4)$ & $5,09(7)$ & 2 \\
\hline \multirow{2}{*}{ Albit } & $\mathrm{Gew} \%^{2}$ & $11(1)$ & $2(2)$ & $0,09(1)$ & $19,6(1)$ & $64,0(5)$ & $96,38(4)$ & 2 \\
& $\mathrm{apfu}^{3}$ & $0,96(12)$ & $0,1(1)$ & $0,004(1)$ & $1,061(1)$ & $2,932(1)$ & $5,073(2)$ & 2 \\
\hline \hline
\end{tabular}

${ }^{1}$ Neben den aufgeführten Elementen wurden teilweise auch geringe Mengen von $\mathrm{Sr}, \mathrm{Fe}$ und $\mathrm{Ba}$ im Bereich der jeweiligen Nachweisgrenzen nachgewiesen $\left(\mathrm{DL}_{\mathrm{Sr}}=\mathrm{DL}_{\mathrm{Fe}}=0,06 \mathrm{Gew} . \%\right.$ und $\mathrm{DL}_{\mathrm{Ba}}=0,05$ Gew.\%).

2 Zahl der Einzelanalysen.

${ }^{3}$ apfu (engl. für atoms per formula unit): Atome pro Formeleinheit bei Normierung der Kationenzahl auf 8 Sauerstoffatome.

qualitativ mit EDX bestimmt und anschließend mit der WDS-Analyse quantifiziert. Sie besteht hauptsächlich aus Al-, La-, Si-, Ti- und Ca-Oxiden (Tab. 9). Die Abweichung der Oxidsumme von $100 \mathrm{Gew} \%$ ist wahrscheinlich auf Korngrenzeneffekte zurückzuführen.

Tab. 9: Chemische Zusammensetzung der Korund-Keramik Vita In-Ceram Alumina aus WDS-Analysen. Alle Angaben sind in Gew.\%. Standardabweichungen der Einzelanalysen vom Mittelwert sind in Klammern angegeben.

\begin{tabular}{l|ccccccccccc|c}
\hline \hline & $\mathrm{Al}_{2} \mathrm{O}_{3}$ & $\mathrm{~K}_{2} \mathrm{O}$ & $\mathrm{TiO}_{2}$ & $\mathrm{FeO}$ & $\mathrm{SiO}_{2}$ & $\mathrm{MgO}$ & $\mathrm{CaO}$ & $\mathrm{MnO}$ & $\mathrm{La}_{2} \mathrm{O}_{3}$ & Summe & $\mathrm{n}^{1}$ \\
\hline \multirow{2}{*}{ Korund } & 96,98 & $<\mathrm{DL}^{2}$ & 0,16 & $<\mathrm{DL}^{2}$ & 0,46 & $<\mathrm{DL}^{2}$ & 0,08 & $<\mathrm{DL}^{2}$ & 1,16 & 98,84 & 8 \\
& $(1,64)$ & & $(0,11)$ & & $(0,37)$ & & $(0,05)$ & & $(0,85)$ & $(0,94)$ & 8 \\
\hline \multirow{2}{*}{ Glasphase } & 39,12 & 0,03 & 4,02 & 0,08 & 14,02 & 0,03 & 1,90 & 0,07 & 34,51 & 93,78 & 5 \\
& $(7,27)$ & $(0,01)$ & $(0,43)$ & $(0,02)$ & $(1,64)$ & $(0,01)$ & $(0,19)$ & $(0,01)$ & $(3,69)$ & $(2,07)$ & 5 \\
\hline \hline
\end{tabular}

${ }^{1}$ Zahl der Einzelanalysen.

$2<\mathrm{DL}$ (detection limit): unterhalb der Nachweisgrenze ( $\mathrm{DL}_{\mathrm{K}_{2} \mathrm{O}}=0,06 \mathrm{Gew} . \%, \mathrm{DL}_{\mathrm{FeO}}=0,03 \mathrm{Gew} . \%$, $\mathrm{DL}_{\mathrm{MgO}}=0,01 \mathrm{Gew} . \%$ und $\mathrm{DL}_{\mathrm{MnO}}=0,03$ Gew.\%).

\section{Degudent U}

Die Goldlegierung Degudent $U$ zeigte eine Entmischung der Matrixphase im Massenkontrastbild (Abb. 42a und b), in der sich eine leistenförmige, hell erscheinende Ausscheidungsphase gebildet hatte. Die chemische Zusammensetzung der Matrix variierte von einer Au-Ag-reichen (heller Bereich) zu einer Pd-Pt-reicheren (dunkler Bereich) Phase (Tab. 10; Abb. 41 und 42b). Die Kristallite der Ausscheidungsphase waren bis zu ca. $15 \mu \mathrm{m}$ lang und erreichten eine Breite bis zu max. $2 \mu \mathrm{m}$. Aufgrund dieser geringen Breite und der unbekannten Tiefe der Kristallite waren reine Analysen an Körnern dieser Ausscheidungsphase nicht möglich. Dennoch konnte eine semiquantitative Aussage über die chemische Zusammensetzung getroffen werden, indem nur Analysen mit einem sehr geringen Goldgehalt berücksichtigt wurden. Das Element Gold 


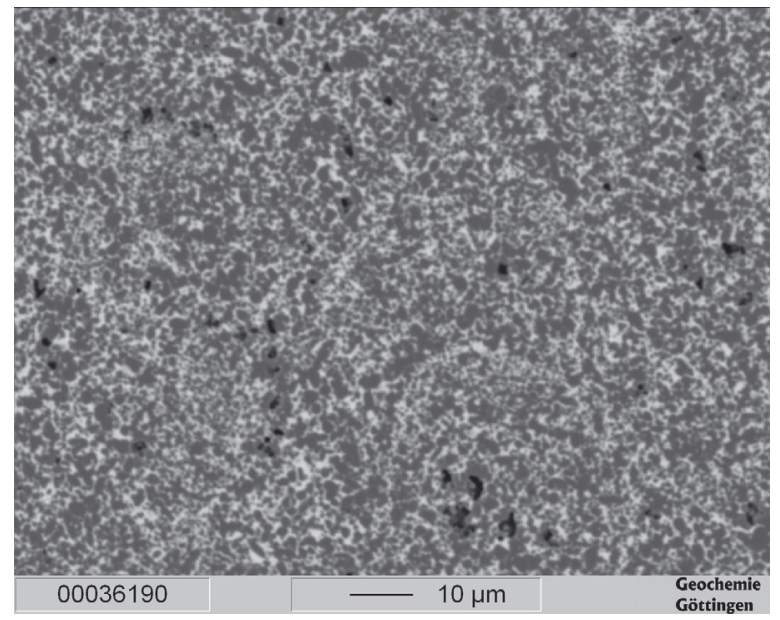

(a) Übersichtsaufnahme (Massenkontraste).

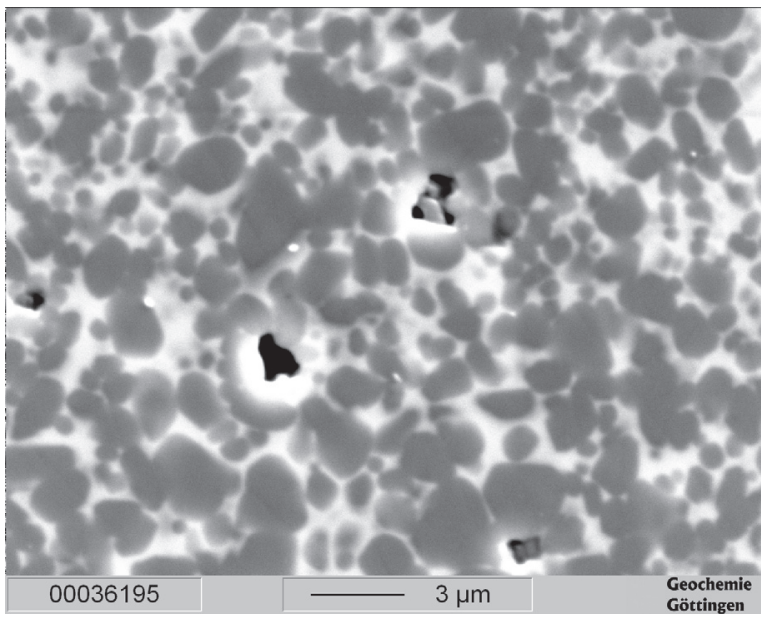

(b) Detailaufnahme (Oberflächenstruktur).

Abb. 40: Elektronenbilder der Korund-Keramik Vita In-Ceram Alumina. Schwarze Korundkörner sind von einer hellen La-Glasmatrix umgeben. Zum Teil sind noch Porenräume erhalten, die im Massenkontrastbild (a) vollständig schwarz erscheinen, während sie im SE-Bild (b) durch einen weißen Überstrahlungssaum gekennzeichnet sind.

kann nämlich in dieser Legierung als Indiz für den Matrixanteil innerhalb einer Analyse genommen werden. In den vorliegenden Analysen lag der gemessene Goldgehalt jedoch nie unter 1 Gew.\%, sodass von einer Mischanalyse der Aussscheidungsphase mit der Matrixphase ausgegangen werden muss. Aufgrund des Fehlens reiner Analysen von dieser Ausscheidungsphase war es sinnvoll, nur Elemente mit einer hohen Massenprozentzahl zu betrachten und Nebenelemente zu vernachlässigen. Unter Berücksichtigung dieser Umstände konnte anhand vier guter Analysen (Au-Gehalt $\approx 2$ Gew.\%) eine chemische Zusammensetzung von $\operatorname{Re}_{2,8} \operatorname{PtIr}_{1,2}$ für diese Ausscheidungsphase bestimmt werden, welche auch röntgenographisch bestätigt wurde (Vgl. Abschnitt 5.4.3.1).

\section{Remanium GM 800+}

Die RE-Bilder der CoCrMo-Legierung Remanium GM 800+ wiesen eine leichte Entmischung der Co-Cr-reichen Matrixphase auf, wobei ein erhöhter Co-Gehalt mit niedrigeren Cr- und MoGehalten einherging (Abb. 42c; Tab. 11). In der Matrix waren ungleichmäßsig gestaltete Kristallite einer Cr-Mo-reichen Ausscheidungsphase (ungefähre chemische Zusammensetzung: $\mathrm{Cr}_{9} \mathrm{Co}_{8} \mathrm{Mo}_{2}$ ) zu erkennen, die bis zu $50 \mu \mathrm{m}$ lang waren und eine Dicke von 0,5-6 $4 \mathrm{~m}$ hatten (Abb. 42c und d).

\section{Wiron 99}

Die Matrix der Ni-Cr-Mo-Legierung variierte zwischen Ni- und Mo-reicheren Bereichen (Abb. 42e; Tab. 12). In der Ni-Cr-Mo-Matrix hatten sich zwei verschiedene Ausscheidungsphasen gebildet (Abb. 42f), von denen eine leistenförmig ausgeprägte Ni-Mo-Cr-Phase den größten Volumenanteil darstellt (max. $10 \mu \mathrm{m}$ lang und bis zu $3 \mu \mathrm{m}$ dick). Neben den Legierungsmetallen ließ sich in dieser Ausscheidungsphase auch eine Anreicherung von Si und Ce beobachten (Tab. 12). 
Tab. 10: Chemische Zusammensetzung der Au-Pd-Pt-Legierung Degudent $U$ aus WDSAnalysen $^{1}$. Alle Angaben sind in Gew.\%. Standardabweichungen der Einzelanalysen vom Mittelwert sind in Klammern angegeben.

\begin{tabular}{l|ccccccccccc|c}
\hline \hline & $\mathrm{Pd}$ & $\mathrm{In}$ & $\mathrm{Au}$ & $\mathrm{Fe}$ & $\mathrm{Sn}$ & $\mathrm{Pt}$ & $\mathrm{Re}$ & $\mathrm{Ag}$ & $\mathrm{Cu}$ & $\mathrm{Ir}$ & $\mathrm{Summe}$ & $\mathrm{n}^{2}$ \\
\hline Matrix, & 8,16 & 1,57 & 77,26 & 0,19 & 0,56 & 9,56 & 0,19 & 1,23 & 0,23 & $\leq D L^{3}$ & 98,98 & 31 \\
gesamt & $(1,16)$ & $(0,36)$ & $(4,44)$ & $(0,06)$ & $(0,20)$ & $(3,11)$ & $(0,06)$ & $(0,17)$ & $(0,04)$ & & $(0,34)$ & 31 \\
\hline Matrix, & 9,80 & 1,72 & 71,42 & 0,27 & 0,46 & 13,81 & 0,19 & 0,99 & 0,18 & $\leq D L^{3}$ & 98,86 & 7 \\
Pd $>9$ & $(0,46)$ & $(0,35)$ & $(2,02)$ & $(0,03)$ & $(0,18)$ & $(1,56)$ & $(0,06)$ & $(0,07)$ & $(0,03)$ & & $(0,47)$ & 7 \\
\hline Matrix, & 6,76 & 1,24 & 82,36 & 0,125 & 0,480 & 6,19 & 0,19 & 1,43 & 0,27 & $\leq D L^{3}$ & 99,06 & 7 \\
Pd $<7$ & $(0,19)$ & $(0,04)$ & $(0,57)$ & $(0,004)$ & $(0,034)$ & $(0,29)$ & $(0,04)$ & $(0,04)$ & $(0,02)$ & & $(0,22)$ & 7 \\
\hline Re-Pt- 4 & 0,38 & $\leq D L^{3}$ & 1,80 & 0,022 & $\leq D L^{3}$ & 17,74 & 53,30 & $\leq D L^{3}$ & 0,30 & 25,75 & 99,30 & \multirow{2}{*}{3} \\
Ir-Phase & $(0,05)$ & & $(0,30)$ & $(0,001)$ & & $(0,18)$ & $(0,24)$ & & $(0,01)$ & $(0,24)$ & $(0,42)$ & 3 \\
\hline \hline
\end{tabular}

${ }^{1}$ Neben den aufgeführten Elementen wurden teilweise sehr geringe Mengen von Ru und Rh im Bereich der jeweiligen Nachweisgrenze gemessen $\left(\mathrm{DL}_{\mathrm{Ru}}=\mathrm{DL}_{\mathrm{Rh}}=0,03 \mathrm{Gew} . \%\right)$.

${ }^{2}$ Zahl der Einzelanalysen.

${ }^{3} \leq D L$ (detection limit): unterhalb bzw. in Höhe der Nachweisgrenze $\left(\mathrm{DL}_{\mathrm{In}}=0,02 \mathrm{Gew} . \%, \mathrm{DL}_{\mathrm{Sn}}=0,02 \mathrm{Gew} . \%\right.$, $\mathrm{DL}_{\mathrm{Ag}}=0,04 \mathrm{Gew} . \%$ und $\left.\mathrm{DL}_{\mathrm{Ir}}=0,08 \mathrm{Gew} . \%\right)$.

${ }^{4}$ Aufgrund der geringen Korngröße sind Mischanalysen der Ausscheidungs- mit der Matrixphase kaum zu vermeiden. Nur vier Einzelanalysen sind nahezu frei von einer Mischanalyse.

Den kleinsten Volumenanteile machte eine Ce-P-haltige, faserig ausgebildete Ausscheidungsphase aus, von der aufgrund der geringen Korngröße (max. $10 \mu \mathrm{m}$ lang und max. 0,7 $\mu \mathrm{m}$ dick) nur Mischanalysen vorlagen. Zudem wich die Summe der Analysen deutlich von 100 Gew.\% ab, was wahrscheinlich durch Korngrenzeneffekte verursacht wurde. 
a)

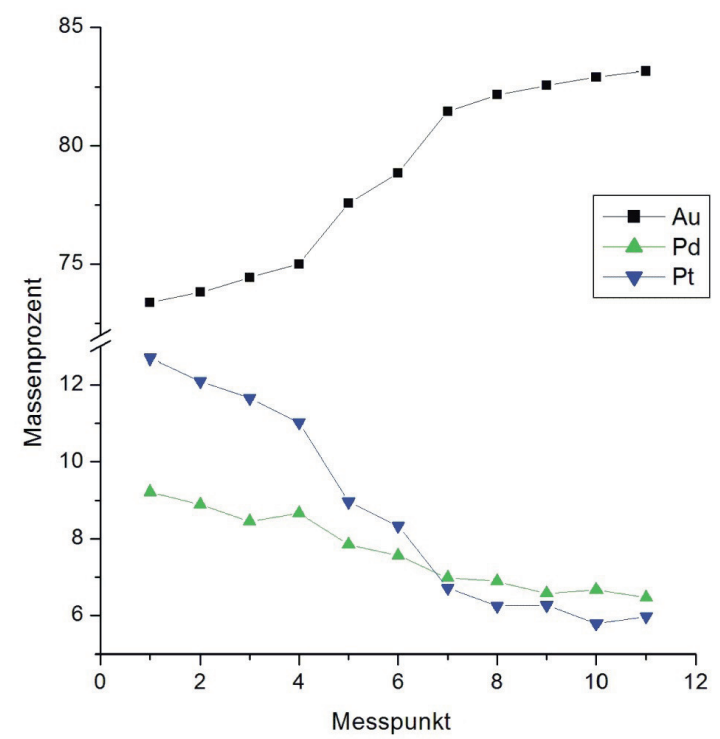

b)

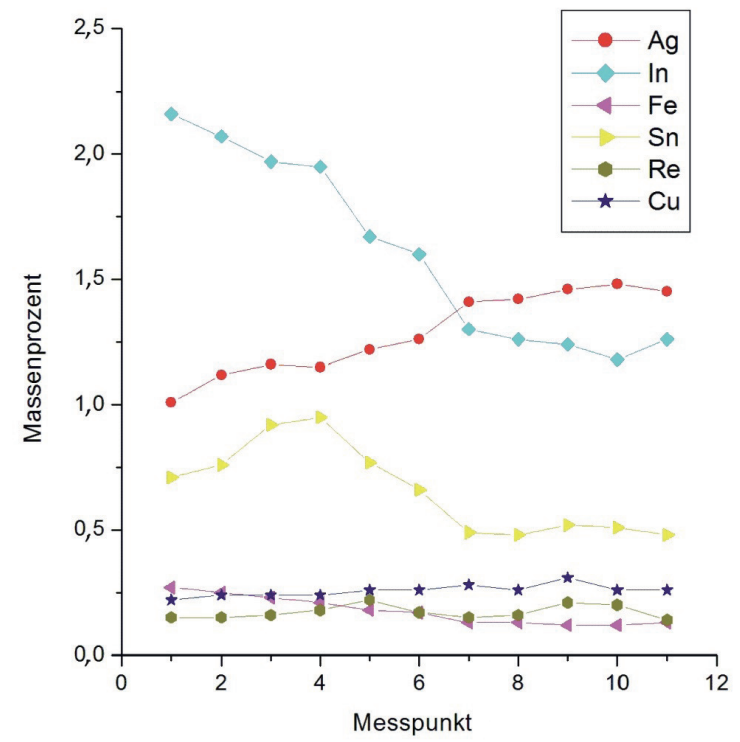

Abb. 41: Linienmessung in der Matrixphase der Goldlegierung Degudent U. Die Messpunkte - mit jeweils 1,5 $\mu \mathrm{m}$ Abstand - beginnen im dunkel erscheinenden Bereich und gehen in den helleren, Au-reicheren Bereich über (Vgl. auch Abb. 42b). a) Verlauf der Massenprozente für die Hauptelemente, b) Verlauf der der Massenprozente für die Nebenelemente.

Tab. 11: Chemische Zusammensetzung der Co-Cr-Mo-Legierung Remanium GM 800+ aus WDS-Analysen ${ }^{1}$. Alle Angaben sind in Gew.\%. Standardabweichungen der Einzelanalysen vom Mittelwert sind in Klammern angegeben.

\begin{tabular}{l|cccccccc|c}
\hline \hline & $\mathrm{Mo}$ & $\mathrm{Si}$ & $\mathrm{Co}$ & $\mathrm{Cr}$ & $\mathrm{Mn}$ & $\mathrm{P}$ & $\mathrm{Fe}$ & Summe & $\mathrm{n}^{2}$ \\
\hline \multirow{2}{*}{ Matrix, gesamt } & 4,94 & 0,52 & 66,05 & 27,19 & 0,44 & $\leq D L^{3}$ & 0,047 & 99,24 & 32 \\
& $(1,01)$ & $(0,06)$ & $(2,32)$ & $(1,23)$ & $(0,04)$ & & $(0,006)$ & $(0,42)$ & 32 \\
\hline Matrix, & 6,13 & 0,59 & 63,35 & 28,62 & 0,48 & $\leq D L^{3}$ & 0,043 & 99,29 & 11 \\
Mo $>6, \mathrm{Cr}>28$ & $(0,19)$ & $(0,02)$ & $(0,66)$ & $(0,24)$ & $(0,02)$ & & $(0,006)$ & $(0,43)$ & 10 \\
\hline Matrix, & 3,83 & 0,45 & 68,52 & 25,76 & 0,40 & $\leq D L^{3}$ & 0,050 & 99,06 & 10 \\
Mo $<4, \mathrm{Cr} \leq 26$ & $(0,14)$ & $(0,02)$ & $(0,57)$ & $(0,21)$ & $(0,01)$ & & $(0,004)$ & $(0,39)$ & 10 \\
\hline Ausscheidungs- & 16,62 & 0,60 & 37,73 & 40,14 & 0,41 & 0,39 & 0,025 & 95,93 & 10 \\
phase & $(0,88)$ & $(0,04)$ & $(2,12)$ & $(1,05)$ & $(0,03)$ & $(0,04)$ & $(0,006)$ & $(0,61)$ & 10 \\
\hline \hline
\end{tabular}

${ }^{1}$ Neben den aufgeführten Elementen wurden teilweise geringe Mengen von W und Ni im Bereich der jeweiligen Nachweisgrenze gemessen $\left(\mathrm{DL}_{\mathrm{W}}=0,06 \mathrm{Gew} . \%\right.$ bzw. $\mathrm{DL}_{\mathrm{Ni}}=0,03 \mathrm{Gew} . \%$ ).

2 Zahl der Einzelanalysen.

${ }^{3} \leq D L$ (detection limit): unterhalb bzw. in Höhe der Nachweisgrenze ( $\left.\mathrm{DL}_{\mathrm{P}}=0,01 \mathrm{Gew} . \%\right)$. 
Tab. 12: Chemische Zusammensetzung der Ni-Cr-Mo-Legierung Wiron 99 aus WDS-Analysen ${ }^{1}$. Alle Angaben sind in Gew.\%. Standardabweichungen der Einzelanalysen vom Mittelwert sind in Klammern angegeben.

\begin{tabular}{l|cccccccc|c}
\hline \hline & $\mathrm{Mo}$ & $\mathrm{Si}$ & $\mathrm{Cr}$ & $\mathrm{Ni}$ & $\mathrm{P}$ & $\mathrm{Ce}$ & $\mathrm{Mn}$ & Summe & $\mathrm{n}^{2}$ \\
\hline \multirow{2}{*}{ Matrix, gesamt } & 9,58 & 1,14 & 21,44 & 64,65 & 0,03 & 0,92 & 0,36 & 98,27 & 13 \\
& $(1,09)$ & $(0,35)$ & $(0,56)$ & $(1,54)$ & $(0,02)$ & $(0,52)$ & $(0,05)$ & $(0,004)$ & 13 \\
\hline Matrix, & 10,96 & 1,57 & 20,82 & 62,78 & 0,010 & 1,57 & 0,42 & 98,30 & 4 \\
$>$ 10 Gew.\% Mo & $(0,71)$ & $(0,28)$ & $(0,67)$ & $(0,90)$ & $(0,003)$ & $(0,48)$ & $(0,03)$ & $(0,29)$ & \\
\hline Matrix, & 8,65 & 0,86 & 21,74 & 66,05 & $\leq D L^{3}$ & 0,54 & 0,31 & 98,35 & 5 \\
$<9$ Gew.\% Mo & $(0,26)$ & $(0,11)$ & $(0,15)$ & $(0,63)$ & & $(0,11)$ & $(0,02)$ & $(0,26)$ & \\
\hline Mo-reiche & 29,87 & 6,39 & 10,55 & 42,73 & 0,03 & 9,17 & 0,17 & 99,09 & \multirow{2}{*}{3} \\
Einschlüsse & $(0,38)$ & $(0,02)$ & $(0,01)$ & $(0,08)$ & $(0,02)$ & $(0,15)$ & $(0,02)$ & $(0,33)$ & \\
\hline Ce-P-reiche & $\leq D L^{3}$ & 0,65 & 5,21 & 8,26 & 31,41 & 32,28 & 0,03 & 77,96 & \multirow{2}{*}{2} \\
Einschlüsse $^{4}$ & & $(0,13)$ & $(1,17)$ & $(2,60)$ & $(2,81)$ & $(1,55)$ & $(0,03)$ & $(0,45)$ & \\
\hline \hline
\end{tabular}

${ }^{1}$ Neben den aufgeführten Elementen wurden teilweise geringe Mengen von $\mathrm{Fe}, \mathrm{Nb}$ und Ti gemessen $(<0,2$ Gew.\%).

2 Zahl der Einzelanalysen.

${ }^{3} \leq D L$ (detection limit): unterhalb bzw. in Höhe der Nachweisgrenze ( $\mathrm{DL}_{\mathrm{Mo}}=0,07 \mathrm{Gew} . \%$, $\mathrm{DL}_{\mathrm{P}}=0,01$ Gew.\%).

4 Aufgrund der geringen Korngröße liegen hier Mischanalysen vor. 


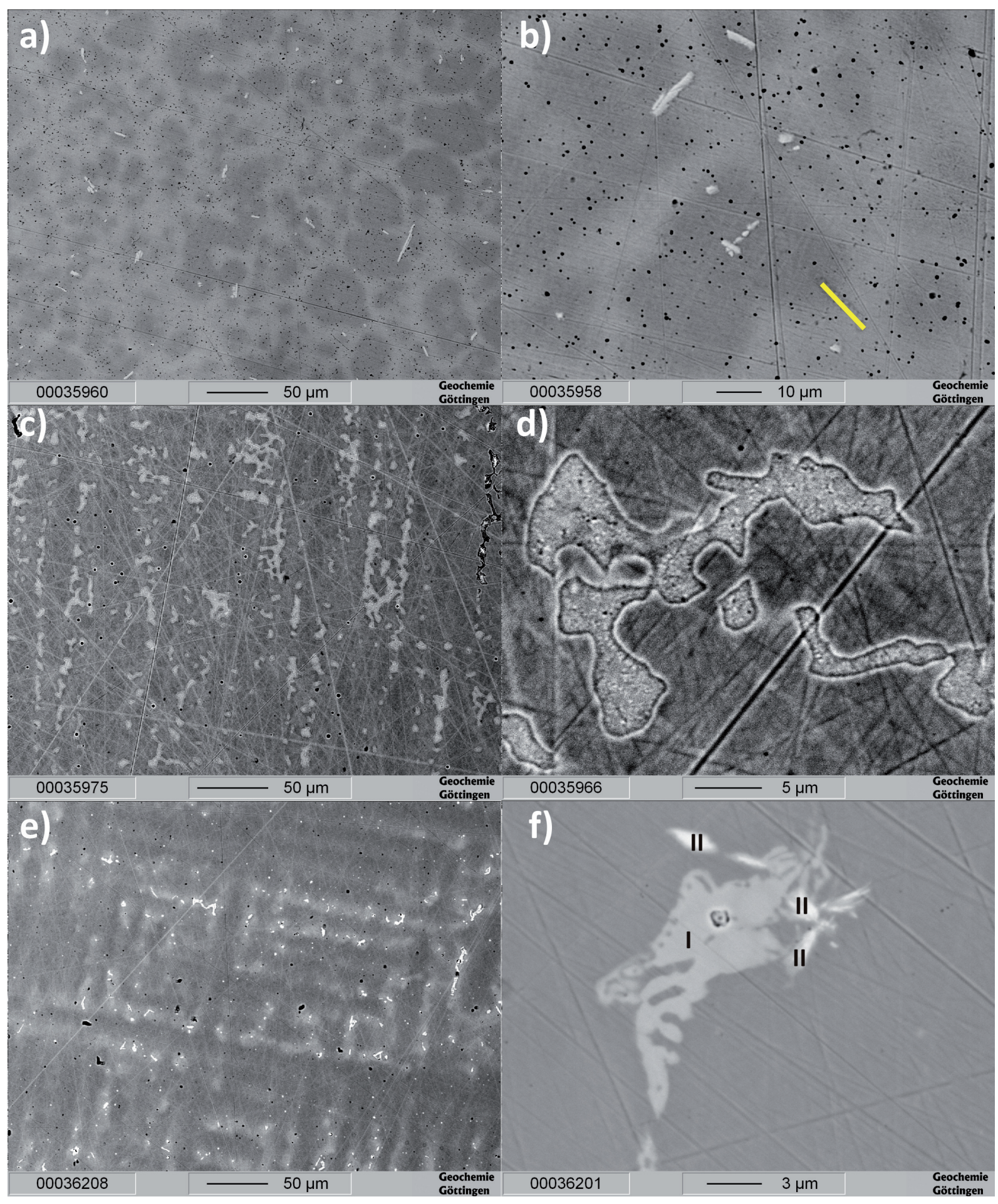

Abb. 42: RE-Aufnahmen der Goldlegierung Degudent $U$ (a, b) und der NEM-Legierungen Remanium GM 800+ (c, d) und Wiron 99 (e, f) in verschiedenen Vergrößerungen. In allen Aufnahmen ist eine leichte Entmischung der Matrix in Bereiche höherer und niedriger atomarer Masse sowie mindestens eine Ausscheidungsphase (hell) zu sehen. Schwarze Stellen deuten auf Porenräume hin. Im Fall der Legierung Wiron 99 sind eindeutig zwei Ausscheidungsphasen, eine Mo-reiche (I) und eine Ce-P-reiche Phase (II), zu erkennen (f). In (b) sind im unteren, rechten Bildbereich die Messpunkte einer Linienmessung (in gelb) eingezeichnet. 


\subsection{Ergebnisse der röntgenographischen Phasenanalyse}

\subsubsection{Phasenanalyse der direkten Füllmaterialien}

\subsubsection{Dentalamalgame Amalcap, regular und fast}

Als Hauptphasen der Dentalamalgame wurden sowohl am PTS-Goniometer als auch an der Synchrotron-Beamline BW5 (HASYLAB) die Phasen $\mathrm{Ag}_{2} \mathrm{Hg}_{3}, \mathrm{Ag}_{3} \mathrm{Sn}$ und $\mathrm{Cu}_{6} \mathrm{Sn}_{5}$ nachgewiesen (s. Tab. 13 und Anhang Abb. B.3). Die Unterschiede bei gleichem Ausgangsmaterial, aber unterschiedlicher Messmethode sind v.a. auf die Unterschiede im Beugungsvolumen zurückzuführen, da sich herausgestellt hatte, dass die Dentalamalgame relativ grobkörnig waren und bei den Messungen am XRD 3000 PTS trotz Probentranslation ein im Verhältnis zur Synchrotronstrahlung kleines Beugungsvolumen erfasst wurde.

Tab. 13: Ergebnisse aus den Rietveld-Verfeinerungen der Dentalamalgame Amalcap regular und fast. Gemessen an der Synchrotron-Beamline BW5 (HASYLAB; $\lambda_{1}=0,1613 \AA$ und $\lambda_{2}=$ $0,1248 \AA)$ und am Röntgendiffraktometer XRD 3000 PTS $(\lambda=1,79 \AA)$. Ausgewertet mit GSAS.

\begin{tabular}{l|cccc|c}
\hline \hline Probe & $R_{w p}[\%]$ & $\mathrm{Ag}_{2} \mathrm{Hg}_{3}[\mathrm{Gew} . \%]$ & $\mathrm{Ag}_{3} \mathrm{Sn}_{\text {[Gew.\%] }}$ & $\mathrm{Cu}_{6} \mathrm{Sn}_{5}$ [Gew.\%] & Messaufbau \\
\hline Amalcap regular & 26,56 & $95,36(6)$ & $4,1(8)$ & $0,6(5)$ & PTS \\
Amalcap regular & 10,68 & $80,9(3)$ & $11,2(7)$ & $7,9(4)$ & BW5 \\
\hline Amalcap fast & 26,39 & $97,60(3)$ & $1,2(5)$ & $1,2(6)$ & PTS \\
Amalcap fast & 4,2 & $74,3(1)$ & $12,4(3)$ & $13,3(4)$ & BW5 \\
\hline \hline
\end{tabular}

\subsubsection{Dentalkomposite, Prothesenkunststoffe und Glasionomerzement}

In allen Fällen wurden breite Beugungsringe der amorphen Bestandteile wie Kunststoff und der Glasfüllpartikel beobachtet (s. Abb. 43 und 44). Nur im Fall der Tetric-Serie konnte neben den amorphen Beugungsringen ein deutlicher Anteil der kristallinen $\mathrm{YbF}_{3}$-Phase detektiert werden (s. Abb. 43b). Daneben wiesen auch der Prothesenkunststoff Bioplus und das Komposit Luxacore Reflexe mit geringer Intensität auf. Im Fall von Luxacore ließen sich diese Reflexe v.a. aufgrund ihrer geringen Intensität keiner Phase zuweisen, während die Reflexe im Beugungsbild von Bioplus auf kristallinem Zirkon $\left(\mathrm{ZrSiO}_{4}, \mathrm{RG}\right.$ : $\left.I 4_{1} / a m d\right)$ beruhen könnten (s. Abb. 44b). Für die weitere Phasenanalyse wurde aus dem Bioplus-Zahn eine ca. 0,5 mm dicke Scheibe herausgesägt und in drei unterschiedlichen Bereichen, die dem Zahnschmelz, dem Dentin und der Zahnwurzel entsprechen, gemessen. In allen Fällen konnte die Zirkon-Phase, die vorher beim Messen der gesamten Probe detektiert wurde, nachgewiesen werden (Abb. 44b). Allerdings war der prozentuale Anteil der Zirkon-Phase im Vergleich zum amorphen Kunststoff sehr gering (genaue Quantifizierung nicht möglich). 


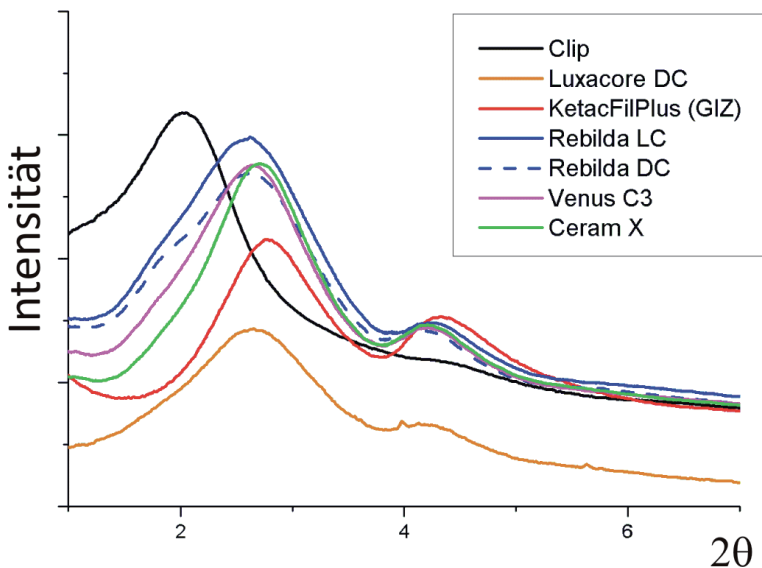

(a) Dentalkomposite und das Glasionomerzement $K e$ tac Fil Plus.

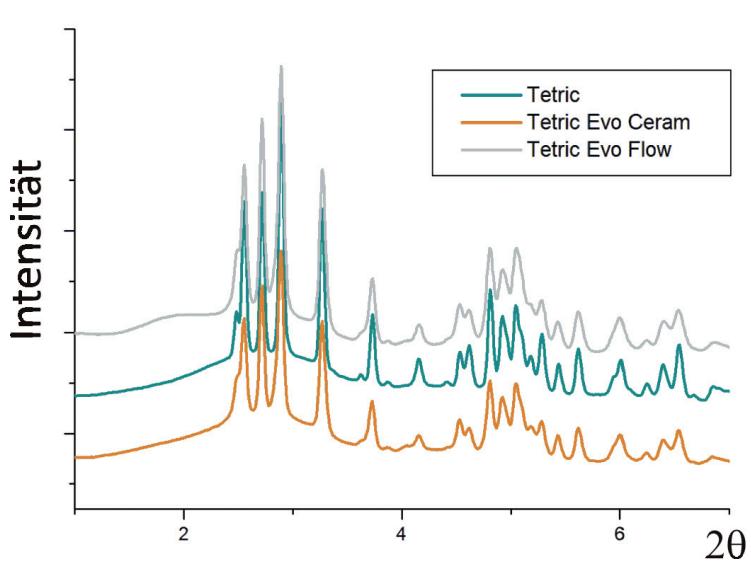

(b) Dentalkomposite der Tetric-Serie mit Reflexen der $\mathrm{YbF}_{3}$-Phase.

Abb. 43: Röntgendiffraktogramme der Dentalkomposite und eines Glasionomerzementes. Aufgenommen an der Beamline BW5 $\left(\lambda_{1}=0,1613 \AA\right.$ und $\left.\lambda_{2}=0,1311 \AA\right)$. Zur besseren Vergleichbarkeit wurden die Beugungsbilder auf $\lambda_{1}$ umgerechnet und die Intensitäten neu skaliert.

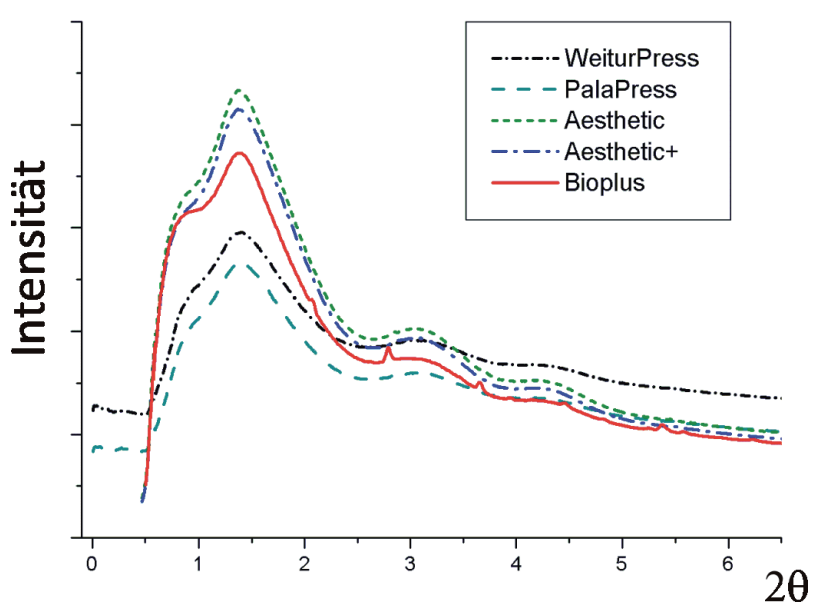

(a) Prothesenkunststoffe. Zur besseren Vergleichbarkeit wurden die Beugungsbilder auf $\lambda_{1}$ umgerechnet und die Intensitäten neu skaliert (Abkürzung „Aesthetic + “ steht für Aesthetic mit erhöhtem Faseranteil).

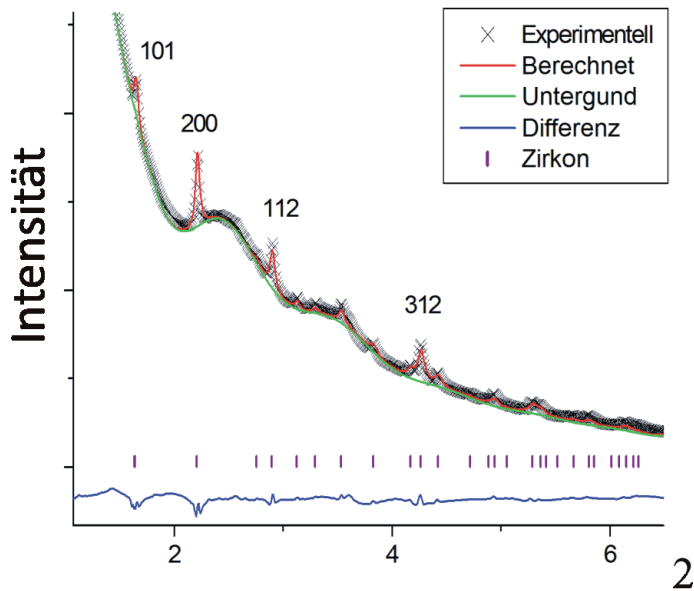

(b) Prothesenkunststoff Bioplus - Wurzelbereich, ausgewertet mit GSAS. Die wichtigsten Reflexe der $\mathrm{ZrSiO}_{4}-\mathrm{Phase}$ sind indiziert.

Abb. 44: Röntgendiffraktogramme der Prothesenkunststoffe. Aufgenommen an der Beamline BW5 $\left(\lambda_{1}=0,1613 \AA, \lambda_{2}=0,1311 \AA\right.$ in (a) und $\lambda=0,1243 \AA$ in (b)). 


\subsubsection{Zinksulfatzemente}

Die Zn-Sulfat-Zemente Cavit und Detaferm, die bei unterschiedlichen Wassersättigungsgraden und verschieden langen Reaktionszeiten untersucht wurden, wurden wegen ihrer geringen Formstabilität nur an der Beamline BW5 gemessen, da an dieser Beamline kurze Messzeiten möglich waren. Je nach Wassersättigungsgrad konnten die kristallinen Phasen Zinkit (ZnO; $\left.P 6_{3} m c\right)$, Gips $\left(\mathrm{Ca}\left[\mathrm{SO}_{4}\right] \cdot 2 \mathrm{H}_{2} \mathrm{O} ; C 2 / c\right)$, Bassanit $\left(\mathrm{Ca}\left[\mathrm{SO}_{4}\right] \cdot 0,5 \mathrm{H}_{2} \mathrm{O} ; P 3_{1} 21\right)$ und ZinksulfatPentahydrat $\left(\left(\mathrm{Zn}(\mathrm{OH})_{2}\right)_{3}\left(\mathrm{ZnSO}_{4}\right)\left(\mathrm{H}_{2} \mathrm{O}\right)_{5} ; P \overline{1}\right)$ nachgewiesen werden (Tab. 14 und 15, Anhang Abb. B.4). Mit zunehmender Reaktionszeit nahmen der Bassanit-Anteil ab und der Gipsanteil zu. Neben dem Zinksulfat-Pentahydrat scheinen sich bei genügend langer Reaktionszeit noch weitere Zinksulfathydrat-Phasen gebildet zu haben, die sich aber nicht eindeutig zuordnen ließen (Anhang Abb. B.4). Im Fall von Detaferm könnte sich z.B. ein Zinksulfat-Trihydrat $\left(\left(\mathrm{Zn}(\mathrm{OH})_{2}\right)_{3}\left(\mathrm{ZnSO}_{4}\right)\left(\mathrm{H}_{2} \mathrm{O}\right)_{3}, R G=I \overline{1}\right.$, Bear et al., 1986) gebildet haben. Diese Mineralphase konnte jedoch aufgrund der starken Reflexüberlagerungen der vorhandenen, niedrig symmetrischen Phasen und des geringen Gewichtsanteils der fehlenden Phase ( $\$ 5$ Gew.\%) nicht sicher bestätigt werden.

Tab. 14: Ergebnisse aus den Rietveld-Verfeinerungen des Zinksulfatzements Detaferm nach verschiedenen Reaktionszeiten in Leitungswasser. Gemessen an der SynchrotronBeamline BW5 $\left(\lambda_{1}=0,1311 \AA\right.$ und $\left.\lambda_{2}=0,1613 \AA\right)$; ausgewertet mit dem Programm GSAS.

\begin{tabular}{lccccc}
\hline \hline \multicolumn{2}{l}{ Reaktionszeit in Leitungswassser } & 30 Min. & 10 Min. $^{1}$ & 10 Min. $^{2}$ & 3 Tage \\
\hline Rwp & {$[\%]$} & 5,09 & 4,62 & 4,01 & 7,00 \\
ZnO & [Gew.\%] & $55,1(2)$ & $43,9(3)$ & $44,8(5)$ & $34,8(2)$ \\
Bassanit & [Gew.\%] & $38,1(6)$ & $25,6(4)$ & $23,4(5)$ & $2,0(3)$ \\
Gips & [Gew.\%] & $3,8(7)$ & $15,4(3)$ & $16,4(7)$ & $35,4(5)$ \\
Zinksulfat-Pentahydrat & [Gew.\%] & $3,1(2)$ & $15,1(7)$ & $15,5(3)$ & $27,8(7)$ \\
\hline \hline
\end{tabular}

${ }^{1}$ Zusätzlich 2 Wochen im Druckverschlussbeutel aus Plastik gelagert.

${ }^{2}$ Zusätzlich 3 Monate im Druckverschlussbeutel aus Plastik gelagert.

Tab. 15: Ergebnisse aus den Rietveld-Verfeinerungen des Zinksulfatzements Cavit nach verschiedenen Reaktionszeiten in Leitungswasser. Gemessen an der Synchrotron-Beamline BW5 $\left(\lambda_{1}=0,1311 \AA\right.$ und $\left.\lambda_{2}=0,1613 \AA\right)$; ausgewertet mit dem Programm GSAS.

\begin{tabular}{lcccc}
\hline \hline \multicolumn{2}{l}{ Reaktionszeit in Leitungswassser } & 30 Min. & 10 Min. ${ }^{1}$ & 3 Tage \\
\hline Rwp & {$[\%]$} & 4,36 & 3,53 & 5,77 \\
ZnO & [Gew.\%] & $52,2(2)$ & $38,7(2)$ & $40,0(3)$ \\
Barit & [Gew.\%] & $26,8(4)$ & $22,5(2)$ & $22,0(3)$ \\
Bassanit & [Gew.\%] & $13,4(5)$ & $7,2(9)$ & $1,2(3)$ \\
Gips & [Gew.\%] & 0 & $9,2(4)$ & $18,1(5)$ \\
Zinksulfat-Pentahydrat & [Gew.\%] & $7,5(2)$ & $22,4(3)$ & $18,7(2)$ \\
\hline \hline
\end{tabular}

${ }^{1}$ Zusätzlich 2 Wochen im Druckverschlussbeutel aus Plastik gelagert. 


\subsubsection{Phasenanalyse der Keramiken}

\subsubsection{Y-ZrO $\mathrm{Z}_{2}$-Keramik Cercon base}

Der Grünling der Cercon base-Keramik bestand zu 96,1(5) Gew.\% aus einer tetragonal stabilisierten $\mathrm{Y}_{-\mathrm{ZrO}_{2}}$-Phase $\left(P 4_{2} / n m c ; a=3,6296(6) \AA, c=5,0692(12) \AA\right)$ und zu 3,9(5) Gew.\% aus einer monoklinen Baddeleyit-Phase $\left(\mathrm{Y}-\mathrm{ZrO}_{2}, P 2_{1} / c ; R_{w p}=6,83 \%, a=5,153(9) \AA\right.$, $b=5,174(10) \AA$ und $c=5,272(10) \AA, \beta=81,1(1) \AA$; Abb 45). Aus dem Grünling wurden runde Plättchen $(d=1 \mathrm{~cm})$ gefräst, die mit und ohne Verblendkeramik endgesintert wurden. Die endgesinterte Cercon base-Keramik bestand zu $100 \mathrm{Gew} . \%$ aus der tetragonal stabilisierten $\mathrm{Y}-\mathrm{ZrO}_{2}$-Phase $\left(R_{w p}=4,98 \%\right)$. In ihrer Verblendkeramik wurde nur Leucit als kristalline Phase nachgewiesen.

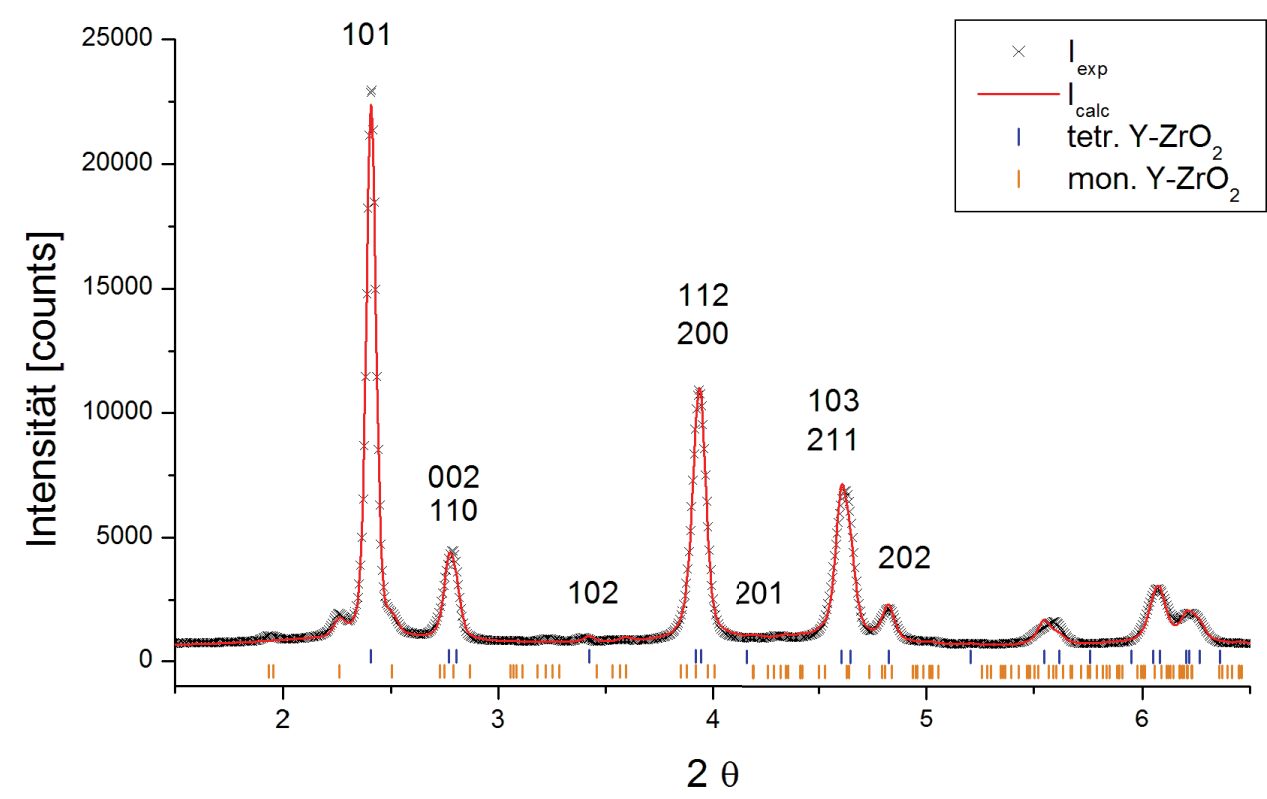

Abb. 45: Röntgendiffraktogramm eines Grünlings der $\mathrm{Y}_{-} \mathrm{ZrO}_{2}-\mathrm{Keramik}$ Cercon Base. Gemessen an der Beamline BW5 $(\lambda=0,1243 \AA)$, ausgewertet mit dem Rietveld-Programm GSAS. Die wichtigsten Reflexe der tetragonal stabilisierten $\mathrm{Y}_{-} \mathrm{ZrO}_{2}$-Phase sind indiziert.

\subsubsection{2 $\mathrm{Li}_{2} \mathrm{Si}_{2} \mathrm{O}_{5}$-Keramik IPS e.max Press}

Die kristallinen Phasen und ihre Anteile wurden sowohl am Rohling als auch an der fertigen Gusskeramik bestimmt. Eindeutig waren in jedem Zustand die Phasen $\mathrm{Li}_{2} \mathrm{Si}_{2} \mathrm{O}_{5}$ und $\mathrm{Li}_{3} \mathrm{PO}_{4}$ vorhanden, die auch in früheren Arbeiten zum Kristallisationsverhalten von P-haltigen LithiumdisilikatGlaskeramiken beobachtet wurden (Höland et al., 2006). Interessant war zu überprüfen, ob auch das Metasilikat $\mathrm{Li}_{2} \mathrm{SiO}_{3}$ vorhanden ist und ob sich weitere Phasen gebildet haben, in denen andere Elemente wie Kalium, Natrium, Zink und Aluminium eingebaut werden können.

$\mathrm{Li}_{2} \mathrm{SiO}_{3}$ konnte sowohl beim Rohling als auch beim Endprodukt (z.T. in geringen Mengen) nachgewiesen werden. Als zusätzliche Phase wurde eine monokline $\gamma_{I I}-\mathrm{Li}_{2} \mathrm{ZnSiO}_{4}$-Phase $\left(P 2_{1} / n\right.$, 
$a=6,262(3) \AA, b=10,602(4) \AA, c=5,021(4) \AA, \beta=90,51(5)^{\circ}$; Yamaguchi et al., 1979) identifiziert. Zinkphosphate bzw. andere Li-Si-O-(P)-Verbindungen konnten nicht nachgewiesen werden. Da die 20-Auflösung am Bruker Smart Apex II im Gegensatz zu den Synchrotron-Beamlines geringer war, wurden bei der Auswertung der Daten vom Bruker Smart Apex II nur die beiden Hauptphasen in der Rietveldverfeinerung berücksichtigt (Tab. 16).

Tab. 16: Ergebnisse aus den Rietveld-Verfeinerungen für IPS e.max Press und IPS e.max $\boldsymbol{C A D}$. Gemessen an den Synchrotron-Beamlines BW5 $\left(\lambda_{1} \approx 0,124 \AA\right)$ und P02.1 (PETRA, $\left.\lambda_{2}=0,2086 \AA\right)$ sowie am Bruker Smart Apex II $(\lambda=0,7093 \AA)$; ausgewertet mit den Programmen GSAS (ohne Textur) und MAUD (mit Texturkorrektur).

\begin{tabular}{|c|c|c|c|c|c|c|c|c|}
\hline Тур & Zustand & $\begin{array}{l}R_{w p} \\
{[\%]} \\
\end{array}$ & $\begin{array}{l}\mathrm{Li}_{2} \mathrm{Si}_{2} \mathrm{O}_{5}{ }^{1} \\
{[\mathrm{Gew} . \%]}\end{array}$ & $\begin{array}{c}\mathrm{Li}_{3} \mathrm{PO}_{4} \\
{[\mathrm{Gew} \%]}\end{array}$ & $\begin{array}{l}\mathrm{Li}_{2} \mathrm{SiO}_{3} \\
{[\mathrm{Gew} . \%]}\end{array}$ & $\begin{array}{c}\mathrm{Li}_{2} \mathrm{ZnSiO}_{4} \\
\text { [Gew.\%] }\end{array}$ & Beamline & Programm $/ \mathrm{n}^{2}$ \\
\hline \multirow{3}{*}{ Press } & Rohling & 4,05 & $83,95(9)$ & $11,10(44)$ & $1,28(54)$ & $3,68(41)$ & BW5 & GSAS / 1 \\
\hline & Rohling & 7,56 & $83,33(8)$ & $11,92(36)$ & $0,94(19)$ & $3,81(25)$ & P02.1 & GSAS / 2 \\
\hline & Mittelwert & & $83,64(44)$ & $11,51(58)$ & $1,11(24)$ & $3,75(9)$ & & \\
\hline \multirow{3}{*}{ Press } & $\begin{array}{l}\text { Gusskanal } \\
\text { (Pulver) }\end{array}$ & 7,76 & $83,7(0,3)$ & $16,3(1,1)$ & - & - & Bruker & GSAS / 1 \\
\hline & $\begin{array}{l}\text { Gusskanal } \\
\text { (ganz) }\end{array}$ & 30,27 & $79,9(23,2)$ & $13,2(3,9)$ & $7,7(2,3)$ & - & P02.1 & $\mathrm{MAUD}^{3} / 1^{*} 41$ \\
\hline & $\begin{array}{l}\text { Scheibe } \\
\text { (verblendet) }\end{array}$ & 9,96 & $81,77(12)$ & $9,68(3)$ & $5,58(2)$ & $2,97(3)$ & BW5 & $\mathrm{MAUD}^{3} / 1^{*} 41$ \\
\hline CAD & Scheibe & 2,97 & $91,18(4)$ & $7,41(45)$ & $0,88(22)$ & $0,53(25)$ & BW5 & GSAS / 1 \\
\hline
\end{tabular}

${ }^{1}$ Monokline Kristallstruktur $C c$ nach Liebau (1961)

${ }^{2}$ Zahl der Messungen $\left(1=\right.$ Single-Shot; $1^{*} 41=1$ Positionshöhe mit 41 Bildern unter verschiedenen $\omega$-Winkeln von $-80^{\circ}$ bis $+80^{\circ}$ in $4^{\circ}$-Schritten)

${ }^{3}$ Phasenanalyse mit Texturberechnung (Texturmodell: E-WIMV, ODF-Auflösung in 3,75-Auflösung)

\subsubsection{3 $\mathrm{Li}_{2} \mathrm{Si}_{2} \mathrm{O}_{5}$-Keramik IPS e.max CAD}

Für die Phasenanalyse von IPS e.max CAD wurde vom Hersteller ein fertig gesintertes Plättchen geliefert, das in Transmissionsstellung am BW5 $(\lambda=0,12389 \AA)$ gemessen wurde. Mit dem Programm X'Pert HighScore Plus wurden eindeutig die Phasen $\mathrm{Li}_{2} \mathrm{Si}_{2} \mathrm{O}_{5}$ und $\mathrm{Li}_{3} \mathrm{PO}_{4}$ identifiziert. Unter der Annahme, dass IPS e.max CAD ähnliche kristalline Phasen wie IPS e.max Press enthielt, wurden auch das Metasilikat $\mathrm{Li}_{2} \mathrm{SiO}_{3}$ und $\mathrm{Li}_{2} \mathrm{ZnSiO}_{4}$ als mögliche Phasen getestet, wobei sich herausstellte, dass nur wenig $\mathrm{Li}_{2} \mathrm{SiO}_{3}$ vorhanden war (Tab. 16). Der Gewichtsanteil der $\mathrm{Li}_{2} \mathrm{ZnSiO}_{4}$-Phase war sogar so gering, dass es im Rahmen eines $3 \sigma$-Fehlers sehr fraglich ist, ob diese Phase in dieser Keramik überhaupt vorlag.

\subsubsection{Feldspatkeramik Vitablocs Esthetic Line}

Die Phasenanalyse dieser Dentalkeramik erwies sich als besonders schwierig, da die Transmissionsaufnahmen zum einen einen hohen Untergrund - durch den Glasanteil in der Keramik verursacht - aufwiesen und zum anderen sehr viele Reflexüberlagerungen niedrig symmetrischer Phasen zeigten. Der Untergrund konnte entweder manuell in GSAS oder durch einen breiten Gauss-Peak in MAUD angepasst werden. Das Problem der Reflexüberlagerungen dagegen ließ sich nur zum Teil lösen, da die Synchrotronbeamlines P02.1 (PETRA) und BW5 (DORIS) eine Beschränkung in ihrer verwendbaren Wellenlänge $\left(\lambda_{P 02.1} \approx 0,2086 \AA\right.$ und $\lambda_{B W 5} \approx$ 
0,124-0,16 §) und der Auflösung durch den vorgegebenen Messaufbau (Probe-Detektor-Abstand und Detektorgröße und -auflösung) aufwiesen. Als Hauptphasen des CAD/CAM-Blocks wurden Nephelin, Sanidin und Mikroklin anhand der hochaufösenden Synchrotronmessung ermittelt. Obwohl Na-haltiger Feldspat (Albit) gemäß der Mikrosondenanalysen sehr wahrscheinlich ist, konnte er röntgenographisch nicht bestätigt bzw. eindeutig von den anderen Feldspatphasen unterschieden werden. Zr- oder Ti-haltige Minerale, wie sie bei der qualitativen EDX-Analyse beobachtet wurden, waren röntgenographisch aufgrund von Reflexüberlagerungen nur schwer zu identifizieren, wobei Rutil mit 5 Gew.\% nachgewiesen werden konnte.

In der Rietveld-Analyse wurden die Gitterparameter der Silikat-Phasen an hochauflösenden Synchrotron-Transmissionsaufnahmen am CAD/CAM-Block verfeinert (Anhang Tab. A.8) und für die weiteren Analysen (pulverisierte Keramik in Glaskapillare) übernommen. Allerdings ist es sehr wahrscheinlich, dass die verfeinerten Gitterparameter eine Mittelung vorhandener Feldspatphasen darstellen. Daher sind die Angaben zu den Phasenanteilen in Tab. 17 nur als eine Näherung zu betrachten.

Tab. 17: Ergebnisse aus den Rietveld-Verfeinerungen für Vitablocs - Esthetic Line. Gemessen an der Synchrotron-Beamline P02.1 (PETRA, $\lambda_{2}=0,2086 \AA$ ) sowie am Bruker Smart Apex II $\left(\lambda_{K \bar{\alpha}}=0,7093 \AA\right)$; ausgewertet mit GSAS bzw. MAUD.

\begin{tabular}{|c|c|c|c|c|c|c|c|}
\hline Zustand & $\begin{array}{c}\text { Rwp } \\
{[\%]}\end{array}$ & $\begin{array}{l}\text { Nephelin } \\
\text { [Gew.\%] }\end{array}$ & $\begin{array}{c}\text { Mikroklin } \\
\text { [Gew.\%] }\end{array}$ & $\begin{array}{c}\text { Sanidin } \\
\text { [Gew.\%] }\end{array}$ & $\begin{array}{c}\text { Rutil } \\
\text { [Gew.\%] }\end{array}$ & $\lambda(\AA)$ & Programm \\
\hline Pulver ${ }^{1}$ & 2,85 & $43(1)$ & $33(3)$ & $24(3)$ & - & 0,7093 & GSAS, Inhouse $^{3}$ \\
\hline Pulver ${ }^{1}$ & 5,8 & $48,1(8)$ & $20(2)$ & $32(2)$ & - & 0,2088 & $\mathrm{GSAS}^{2}$ P02.1 \\
\hline CAD/CAM-Block & 11,3 & $40,04(10)$ & $34,14(9)$ & $21,00(6)$ & $4,82(2)$ & 0,2088 & $\mathrm{MAUD}_{,}^{2,4} \mathrm{P} 02.1$ \\
\hline $\begin{array}{l}{ }^{1} \text { in Glaskapillare } \\
{ }^{2} \text { Gitterparameter } \\
\text { Transmissionsau } \\
\text { Rietveld-Verfeine } \\
{ }^{3} \text { Laborröntgenger } \\
{ }^{4} \text { Phasenanalyse } \\
\text { WIMV, ODF in }\end{array}$ & $\begin{array}{l}\text { aller } \\
\text { ahem } \\
\text { ungen } \\
\text { Bruke } \\
\text { t Verf } \\
\text { - Auflc }\end{array}$ & $\begin{array}{l}\text { Phasen } \\
\text { am CAD/ } \\
\text { übernommer } \\
\text { S Smart AP } \\
\text { einerung de } \\
\text { sung) }\end{array}$ & $\begin{array}{l}-\quad \text { ausgeno } \\
\text { CAM-Block } \\
\text { EX II } \\
\\
\text { Gitterpara }\end{array}$ & $\begin{array}{cc}\text { nmen } & R u \\
\text { mit } & M A U\end{array}$ & $\begin{array}{l}\text { til } \\
\mathrm{D}\end{array}$ & $\begin{array}{l}\text { nhand } \\
\text { ert und } \\
\text { rechnung }\end{array}$ & $\begin{array}{l}\text { der Synchrotron- } \\
\text { für alle weiteren } \\
\text { (Texturmodell: E- }\end{array}$ \\
\hline
\end{tabular}

\subsubsection{Glasinfiltrationskeramik Vita In-Ceram Alumina}

Die Phasenanalyse am Grünling der Vita In-Ceram Alumina-Keramik wurde sowohl am vollständigen CAD/CAM-Block als auch an einer $3 \mathrm{~mm}$ dicken Scheibe vom oberen Ende des CAD/CAMBlockes durchgeführt (Anhang Tab. A.9). Die Gesamtzusammensetzung des Grünlings entsprach 99,05(12) Gew.\% Korund $(R \overline{3} c)$ und 0,95(12) Gew.\% Spinell $\left(\mathrm{Al}_{2} \mathrm{MgO}_{4} ; F m \overline{3} m\right)$. Die Scheibe wurde anschließend mit La-Al-Si-Glas bei ca. $1100^{\circ} \mathrm{C}$ infiltriert und erneut röntgenographisch untersucht. Die Spinell-Phase war nach der Glasinfiltration nicht mehr vorhanden bzw. aufgrund des erhöhten Untergundes nicht mehr detektierbar (Abb. 46).

\subsubsection{Glasinfiltrationskeramik Vita In-Ceram Zirkonia}

Sowohl am ganzen CAD/CAM-Block als auch an einer $3 \mathrm{~mm}$ dicken Scheibe vom Kopfende desselben wurde eine röntgenographische Phasenanalyse durchgeführt. An den verschiedenen Messpunkten wurden keine unterschiedlichen Zusammensetzungen festgestellt. Dieser Grünling 

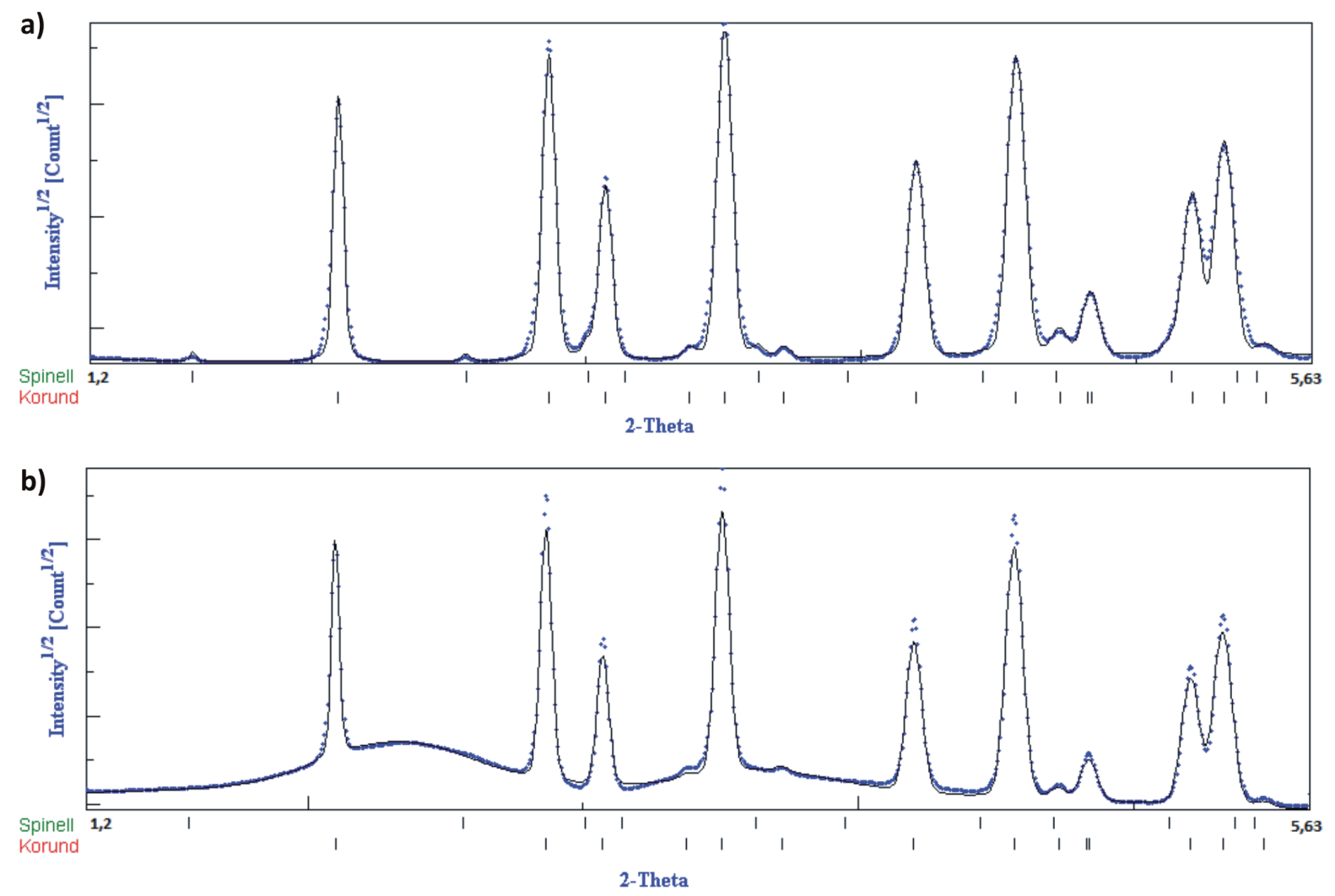

Abb. 46: Röntgendiffraktogramm der Korund-Keramik Vita In-Ceram Alumina. Gemessen an der Beamline BW5 $(\lambda=0,12784 \AA)$, ausgewertet mit dem Programm MAUD. (a) vorgesinterter CAD/CAM-Block (Phasenanteile: 99,05(12) Gew.\% Korund und 0,95(12) Gew.\% Spinell), (b) La-Glas-infiltrierte Scheibe (Phasenanteil der Spinell-Phase geht bei Verfeinerung gegen 0 Gew.\%).

bestand insgesamt zu 65,0(0,4) Gew.\% aus Korund $(R \overline{3} c)$, zu 33,2(0,4) Gew.\% aus tetragonalem $\mathrm{Ce}-\mathrm{ZrO}_{2}$-t $\left(P 4_{2} / n m c\right)$ und zu $1,9(0,4)$ Gew.\% aus monoklinem Ce- $\mathrm{ZrO}_{2}-\mathrm{m}\left(P 2_{1} / c, R_{w p}=6,31 \%\right.$; Tab. A.10). Nach der Glasinfiltration bei $1140^{\circ} \mathrm{C}$ ergaben sich für die kristallinen Phasenanteile 67,6(0,5) Gew.\% Korund, 29,2(2,0) Gew. Ce-ZrO ${ }_{2}$-t sowie 3,3(2,5) Gew.\% Ce-ZrO $\mathrm{Zr}_{2}$ (Anhang Tab. A.10).

\subsubsection{Phasenanalyse der Dentalmetalle und Dentallegierungen}

\subsubsection{Goldlegierung Degudent $U$}

Die Phasenanalyse dieser Au-Legierung wurde einerseits an einer fertigen Gussscheibe mit aufgebrannter Verblendkeramik Duceram Kiss und andererseits an einem Gusskanal vorgenommen. Als kristalline Phase der reinen Verblendkeramik wurde Leucit (RG: $I 4_{1} / a ; a=13,120(5) \AA$ und $c=13,692(5) \AA)$ festgestellt. Des Weiteren war ein nicht weiter differenzierbarer Glasanteil vorhanden, dessen geschätzter Gewichtsanteil deutlich über 60 Gew.\% lag (siehe auch Anhang Abb. B.8). 


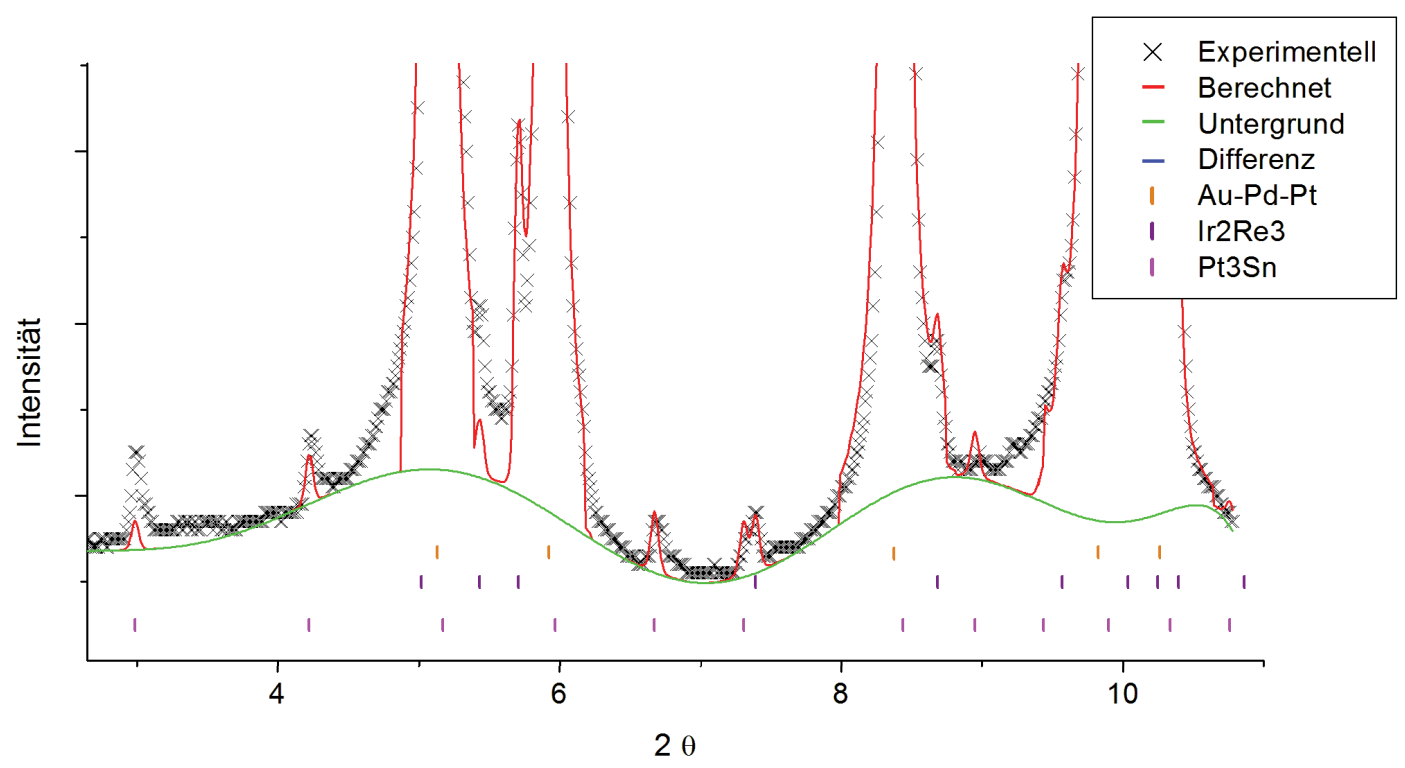

Abb. 47: Ausschnitt aus einem Röntgendiffraktogramm der Au-Pd-Pt-Legierung Degudent $U$ mit Fokus auf den Ausscheidungsphasen (P02.1 - PETRA, $\lambda=0,2084 \AA$ ). Verfeinerte Phasen: $\mathrm{Au}_{70,75} \mathrm{Pd}_{0,15} \mathrm{Pt}_{0,10}(F m \overline{3} m, a=4,0384(3) \AA), \operatorname{Ir}_{2} \operatorname{Re}_{3}\left(P 6_{3} / m m c\right.$, $\left.a_{\text {Ir } 2 \text { Re } 3}=2,753(3) \AA, c_{I r 2 R e 3}=4,405(10) \AA\right)$ sowie $\mathrm{Pt}_{3} \operatorname{Sn}\left(P m \overline{3} m, a_{P t 3 S n}=4,0083(9) \AA\right)$.

Die fertige, mit Keramik verblendete Gussscheibe der Goldlegierung war leicht grobkörnig und zeigte eine geringe Kornstatistik sowie einen durch den Keramikanteil erhöhten Untergrund. Daher wurde für die weitere Phasenanalyse eine Scheibe aus dem Gusskanal gesägt. Die Phasenidentifikation mit X'Pert HighScore Plus erwies sich auch an der Gusskanalscheibe als schwierig, da der prozentuale Anteil der Nebenphasen sehr gering ausfiel (insgesamt $<5$ Gew.\%) und Zweibzw. Dreiphasensysteme nur eingeschränkt Hinweise auf mögliche Legierungsverbindungen geben konnten. Eindeutig geht jedoch aus den Phasendiagrammen hervor, dass eine gute Mischbarkeit zwischen Gold, Silber und Palladium (RG: $F m \overline{3} m$ ) vorliegt, wobei sich auch in diesem einfachen Dreiphasensystem bei tieferen Temperaturen $\left(<850^{\circ} \mathrm{C}\right)$ Überstrukturen wie $\mathrm{Au}_{3} \mathrm{Pd}(\mathrm{RG}: P m \overline{3} m$; Prince, 2006) bilden können. Platin kann einer Au-Pd-Legierung bis zu etwa 25 Gew.\% ohne Änderung des Strukturtyps oder Auftreten von Entmischungseffekten hinzulegiert werden (Harmelin, 2006). Daher wurde für die Hauptphase eine kubisch-flächenzentrierte $\mathrm{Au}_{0,75} \mathrm{Pd}_{0,15} \mathrm{Pt}_{0,10^{-}}$ Phase angenommen, deren Hauptbestandteile denen der Herstellerangaben entsprechen. Der verfeinerte Gitterparameter von $a=4,0384(3) \AA$ deutete ebenfalls auf eine Legierung dieser Elemente in ähnlicher Zusammensetzung hin (Harmelin, 2006). Mit dem Rietveld-Programm GSAS (Larson \& Van Dreele, 1994; Toby, 2001) konnten als Nebenphasen mit ca. 0,35(5) Gew.\% $\mathrm{Ir}_{2} \mathrm{Re}_{3}$ (RG: $P 6_{3} / m m c ; a=2,7545 \AA, c=4,3739 \AA$, Rudman, 1967), wobei auch Pt anstelle von Ir in diese Kristallstruktur eingebaut werden kann, sowie mit ca. 3,9(2) Gew.\% $\mathrm{Pt}_{3} \mathrm{Sn}$ (RG: $P m \overline{3} m, a=4,0005 \AA$; Charlton et al., 1970) identifiziert werden (Verfeinerte Gitterparameter: $a_{\text {Ir } 2 R e 3}=2,753(3) \AA, c_{I r 2 R e 3}=4,405(10) \AA$ sowie $\left.a_{P t 3 S n}=4,0083(9) \AA\right)$. Diese Nebenphasen erklären alle auftretenden Reflexe, die an der Scheibe aus dem Gusskanal gemessen wurden (Abb. 47). 


\subsubsection{Goldlegierung Degulor $M$}

Es wurden röntgenographische Untersuchungen sowohl an einer Scheibe aus dem Gusskanal als auch am pulverisierten Gusskanal vorgenommen. Aufgrund ihrer - im Vergleich zu Degudent $U$ deutlich veränderten - chemischen Zusammensetzung (s. Tab. 6) ist hier auch ein anderes Ausscheidungsverhalten zu erwarten. Die Hauptelemente $\mathrm{Au}, \mathrm{Ag}, \mathrm{Cu}$ und Pt kristallisieren in einer kubisch-flächenzentrierten Struktur, unterscheiden sich jedoch deutlich in ihren Atomgrößen und in den Gitterparametern der reinen Metalle $\left(a_{A u}=4,0857 \AA, a_{A q}=4,0782 \AA, a_{C u}=3,6146 \AA\right.$ und $a_{P t}=3,9236 \AA$; Prince, 2006; Bochvar et al., 2006). Aufgrund der großen Unterschiede in den Gitterparametern ist es wahrscheinlich, dass sich diese Metalle entweder in zwei Phasen gleicher Kristallstruktur, aber unterschiedlichen Gitterparametern entmischen oder Überstrukturen bilden. Zur Vereinfachung seien hier vorerst nur die Hauptelemente $\mathrm{Au}, \mathrm{Ag}$ und $\mathrm{Cu}$ betrachtet, die zusammen 91,1 At.\% der Legierung ausmachen. Auf 100 At.\% normiert ergeben sich 57,4 At.\% Au, 20,1 At.\% Ag und 20,4 At.\% Cu. Eine solche Legierung besteht im thermodynamischen Gleichgewicht bei $300^{\circ} \mathrm{C}$ aus zwei Phasen (Prince, 2006; Bochvar et al., 2006), die auch in der vorliegenden Probe nachgewiesen werden konnten: $\alpha$-Au-Ag-Cu $(F m \overline{3} m, a=3,99(1) \AA$, frei verfeinert) und $\mathrm{AuCu}(P 4 / \mathrm{mmm}, a=2,815 \AA$ und $c=3,72 \AA$; Chen et al., 1982). Diese Beobachtung stimmt auch mit früheren Untersuchungen an dentalen Goldlegierungen überein (Yasudo, 1987; Tani et al., 1991).

Allerdings waren noch weitere, nicht indizierte Reflexe im Röntgenbeugungsbild erkennbar (Abb. 48), die keiner der genannten Phasen zugeordnet werden konnten. Das Programm X'Pert HighSore Plus schlug als zusätzliche Phase $\mathrm{AgZn}_{3}$ vor $\left(P 6_{3} / m m c, a=2,8231(2) \AA, a=4,4407(3) \AA\right.$; Henderson \& Wilcox, 1964), das anhand der Reflexe auch vielversprechend erschien, aber thermodynamisch in einem reinen Ag-Zn-System mit relativ geringem Zn-Anteil (13 At.\% Zn in Degulor $M$ bei Umrechnung auf reines Ag-Zn-System) nicht zu erwarten ist (Gomez-Acebo, 1998). Ein ähnliches Problem stellte sich für eine AuZn-Phase (Pm $\overline{3} m, a=3,14 \AA$; Ipser et al., 1983). Insgesamt ließ sich der Anteil an Ausscheidungsphasen auf max. 5 Gew.\% einschätzen. 


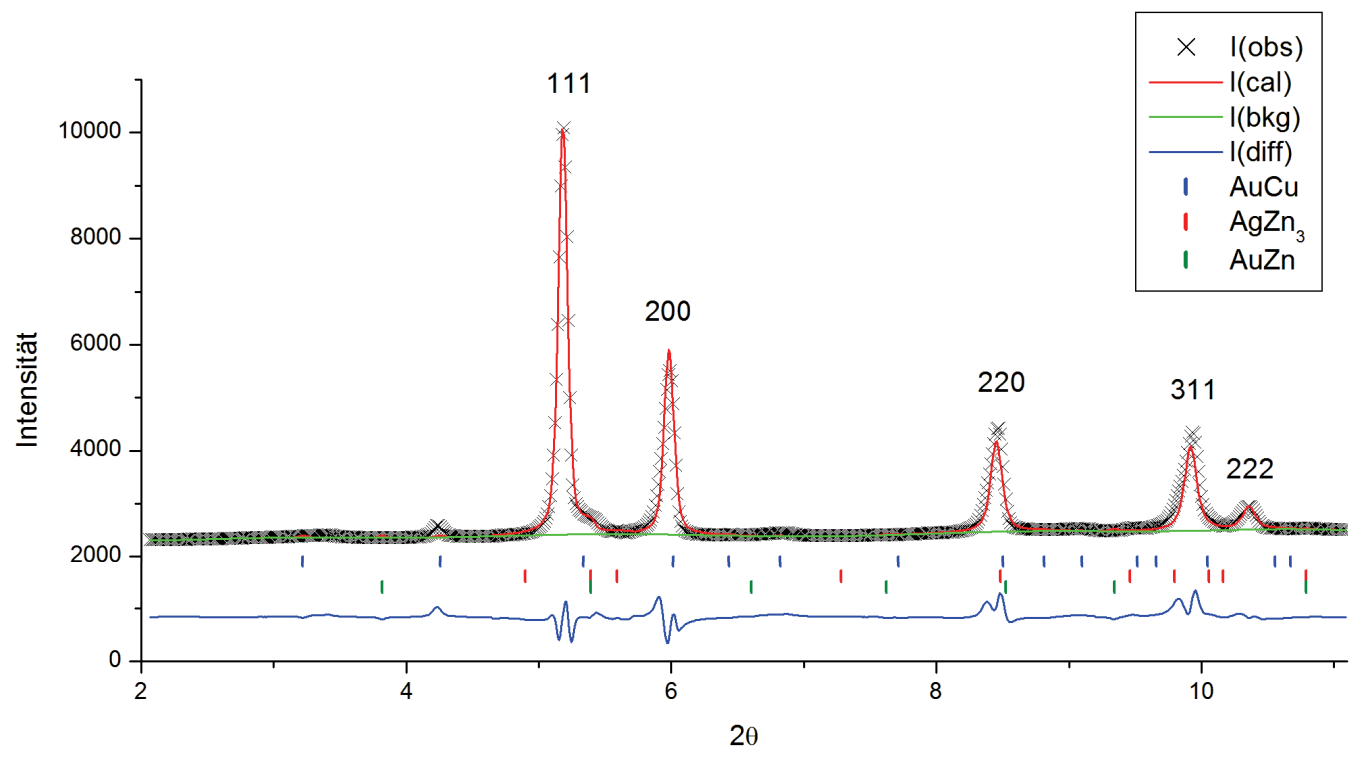

(a) Aus 10 Einzelmessungen aufsummiertes Röntgendiffraktogramm.

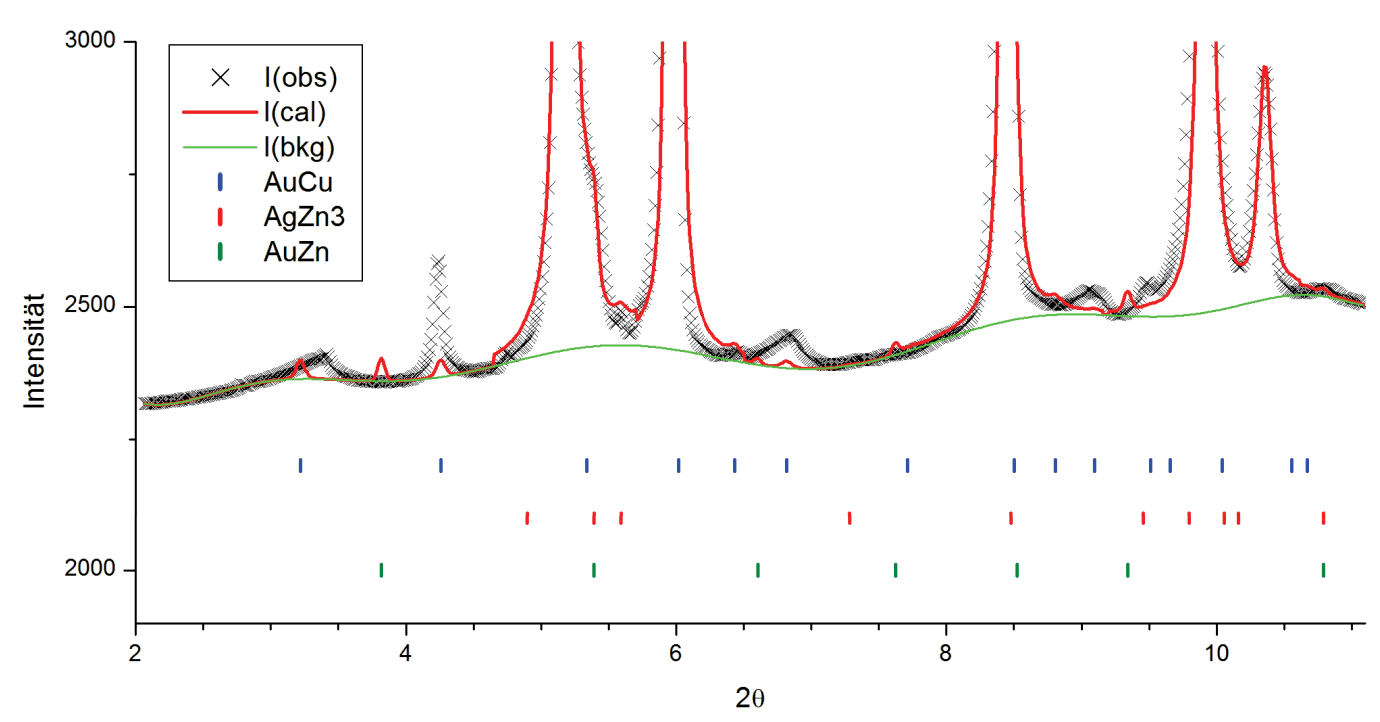

(b) Ausschnitt.

Abb. 48: Scheibe aus dem Gusskanal der hochgoldhaltigen Legierung Degulor $M$ (gemessen am P02.1 - PETRA, $\lambda=\mathbf{0 , 2 0 8 6} \AA$ ). Verfeinerte Phasen: 95,95(2) Gew.\% Au-Ag-Cu ( $F m \overline{3} m$; Reflexe indiziert), 2,3(6) Gew.\% AuCu $(P 4 / m m m, a=2,815 \AA$ und $c=3,72 \AA$; Chen et al., 1982), 0,6(8) Gew.\% $\mathrm{AgZn}_{3}\left(P 6_{3} / m m c, a=2,8231(2) \AA, a=4,4407(3) \AA\right.$ Henderson \&

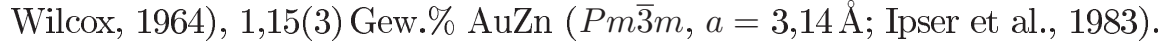




\subsubsection{Titan-Wurzelstift der Firma Gebr. Brasseler}

Bei dem Titan-Wurzelstift wurde eine stark texturierte $\alpha$-Ti-Phase (RG: $P 6_{3} / m m c$ ) mit den Gitterparametern $a=2,95(2) \AA$ und $b=4,677(3) \AA$ detektiert (Abb. 49a; Vgl. Abschnitt 5.6.11). Falls die Titanoberfläche zusätzlich chemisch behandelt wurde oder sich Ti-Oxide oberflächlich gebildet haben, ist ihr Gewichtsanteil im Verhältnis zum reinen Titan so gering, dass sie bei einer Transmissionsaufnahme mit Synchrotronstrahlung mittig durch die Probe (Dicke: 1,5-2,0 mm) nicht nachgewiesen werden können.
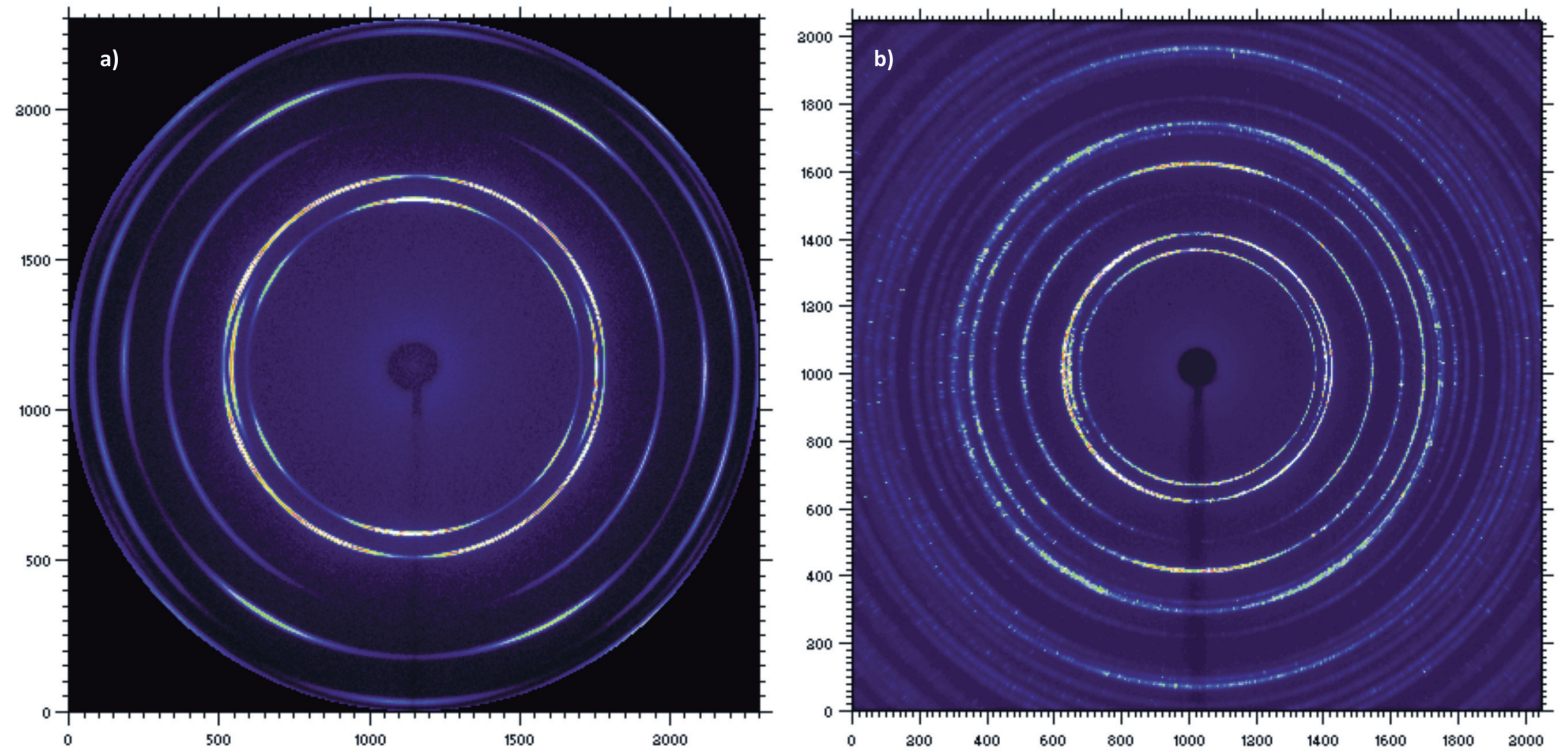

Abb. 49: 2d-Beugungsbilder des Titan-Wurzelstiftes der Firma Gebr. Brasseler (a) und des Titan-Implantats OsseoSpeed der Firma $\boldsymbol{A S T R A ( b ) . ~ I n ~ b e i d e n ~ A u f n a h m e n ~ s i n d ~}$ Intensitätsmaxima auf den Beugungsringen zu erkennen. Das Titanimplantat ist etwas grobkörniger, was aus den gepunkteten Debye-Scherrer-Ringen ersichtlich wird. Beamline BW5 $\left(\lambda_{(a)}=0,1613 \AA, \lambda_{(b)}=0,1243 \AA\right)$.

\subsubsection{Titan-Implantat OsseoSpeed}

Wie beim Titan-Wurzelstift der Firma Gebr. Brasseler wurde auch hier nur eine stark texturierte $\alpha$-Ti-Phase (RG: $P 6_{3} / m m c$ ) nachgewiesen (Abb. 49b; Vgl. Abschnitt 5.6.12), deren Gitterparameter Werte von $a=2,9504(3) \AA$ und $b=4,6861(9) \AA$ aufwiesen. Anhand der gepunkteten DebyeScherrer-Ringe ist zusätzlich (qualitativ) zu erkennen, dass die $\alpha$-Titan-Kristallite im Verhältnis zu den Kristalliten des Titan-Wurzelstiftes eine größere durchschnittliche Korngröße besitzen (Abb. 49). 


\subsubsection{Ni-Cr-Mo-Legierung Wiron99}

Von dieser NEM-Legierung wurden zunächst Synchrotronbeugungsbilder vom Gusskanal aufgenommen. Dabei zeigte sich, dass die durchschnittliche Kristallitgröße des Gusskanals für eine Phasenanalyse zu groß war: Einzelne Reflexe überstrahlten zum Teil Reflexe anderer Kristallite und die Kornstatistik war insgesamt nicht ausreichend (Abb. 50a). Daher wurde der Gusskanal mittels einer Flachfeile pulverisiert und (in Glaskapillaren gefüllt) erneut gemessen. Die Kornstatistik und die Kristallitgröße des Pulvers erwiesen sich für eine weitere Phasenanalyse als geeignet (s. Anhang Abb. B.6).
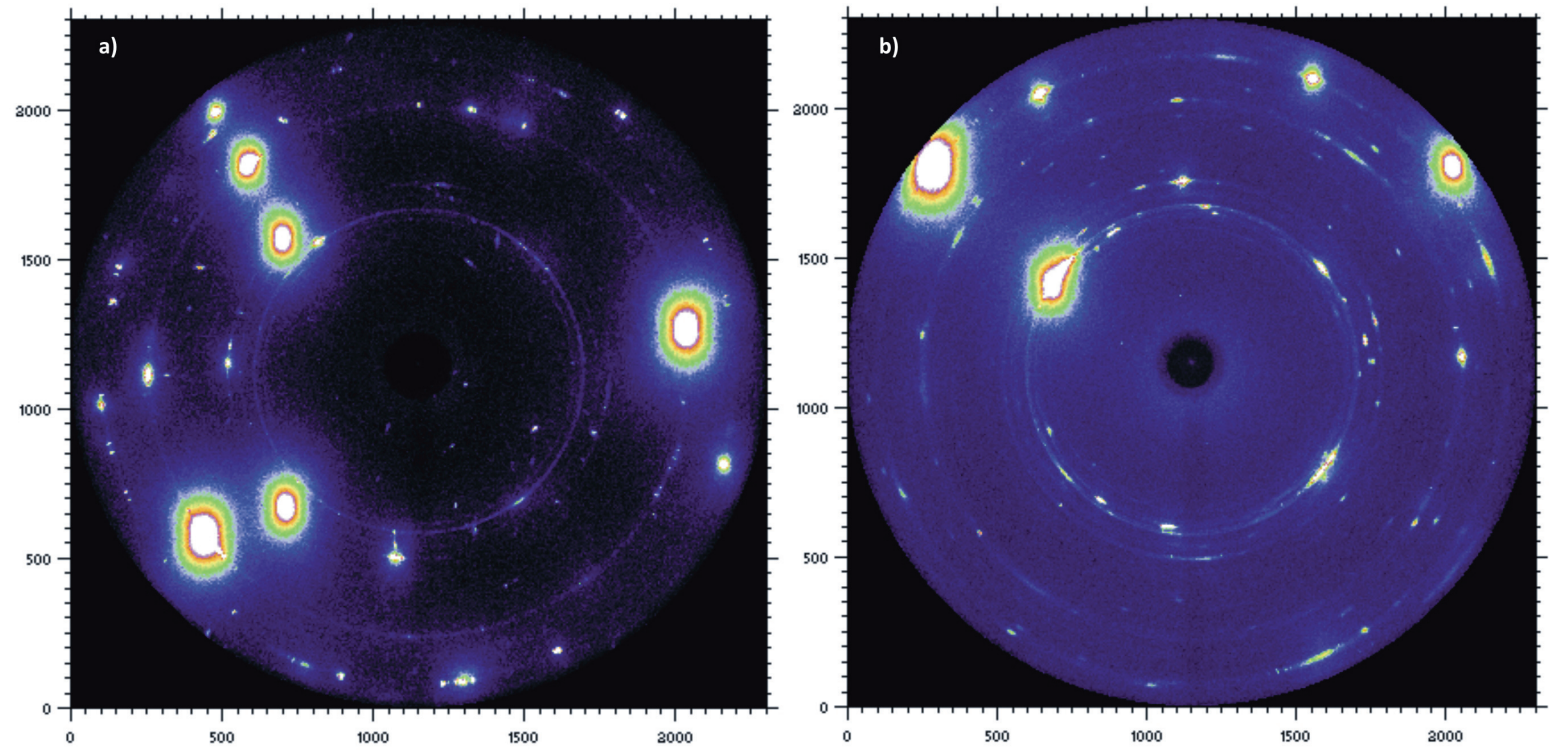

Abb. 50: 2d-Beugungsbilder von Wirong9 (a) und Remanium GM 800+ (b). Einzelne gemessene Kristallite sind so groß, dass es zu Überstrahlungseffekten einzelner Reflexe kommt. Beamline W2 $(\lambda=0,12606 \AA)$.

Als Hauptphase konnte eine Mo-Cr-haltige Ni-Phase $(F m \overline{3} m, a=3,5828(5) \AA)$ nachgewiesen werden, was den Erwartungen anhand der chemischen Gesamtanalyse und des Phasendiagramms für das Cr-Mo-Ni-System von Gupta (2002) entspricht. Die leichten Entmischungen in der Matrix, die in den Massenkontrast-Bildern an der Mikrosonde zu sehen waren (Vgl. Abschnitt 5.3), scheinen nicht mit einem neuen Kristallstrukturtyp einherzugehen. Neben der Matrix-Phase wurden an der Mikrosonde zwei weitere Ausscheidungsphasen - eine Ni-Mo-reiche und eine Ce-P-reiche Phase - bestimmt, deren Chemismus und Kristallstruktur röntgenographisch jedoch nicht bestimmt werden konnte. Als Mo-Ni-reiche Ausscheidungsphase wäre eine $\mathrm{MoNi}_{4}$-Phase möglich $(I 4 / m, a=5,72 \AA$ und $b=3,564 \AA$, Harker, 1944). Bei freier Verfeinerung der Gitter- und der Profilparameter in GSAS ging der Phasenanteil dieser Phase gegen 7,2(8) Gew.\%, wobei die Gitterparameter Werte von $(a=5,754(3) \AA$ und $b=3.496(2) \AA)$ annahmen $\left(R_{w p}=11,0 \%\right.$; Abb. 51). Allerdings konnten durch diese Phase nicht alle vorhandenen Reflexe erklärt werden. Das legte die Schlussfolgerung nahe, dass diese Phase entweder nicht korrekt oder mindestens eine weitere, nicht identifizierbare Phase vorhanden war. Außerdem wurden sowohl CeP als reine CeP-Verbindung sowie verschiedene Ni-haltige Ce-P-Verbindungen getestet, wobei bei freier Verfeinerung die Phasenanteile dieser Phasen gegen 0 Gew.\% gingen. 
a)

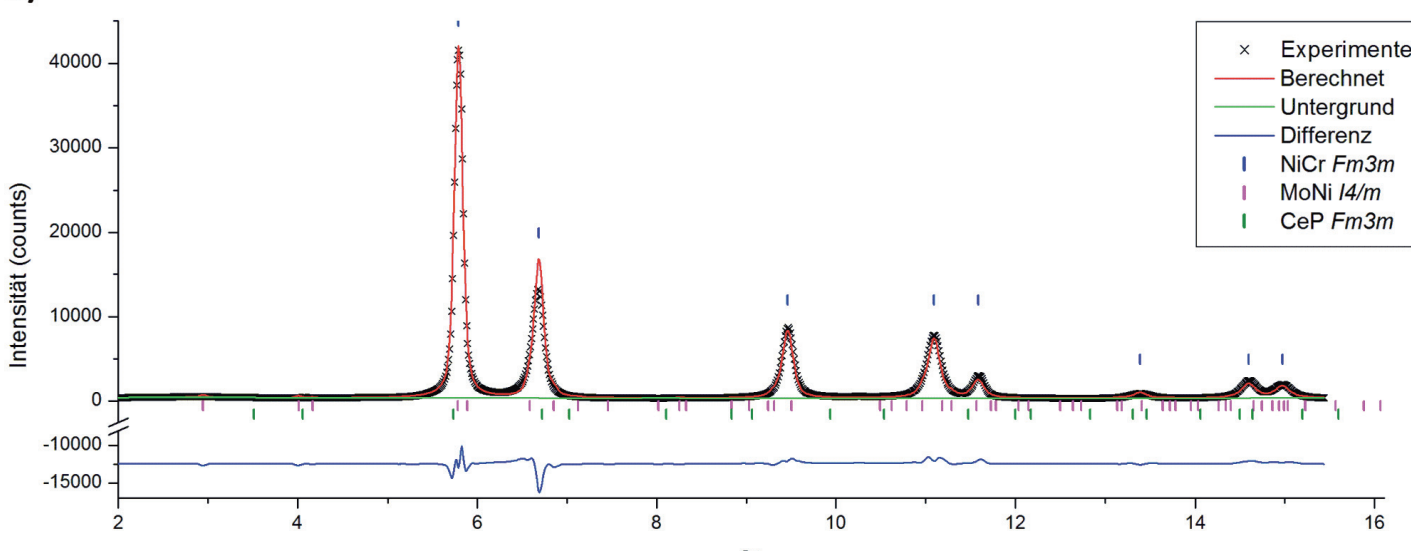

b)

$2 \theta$

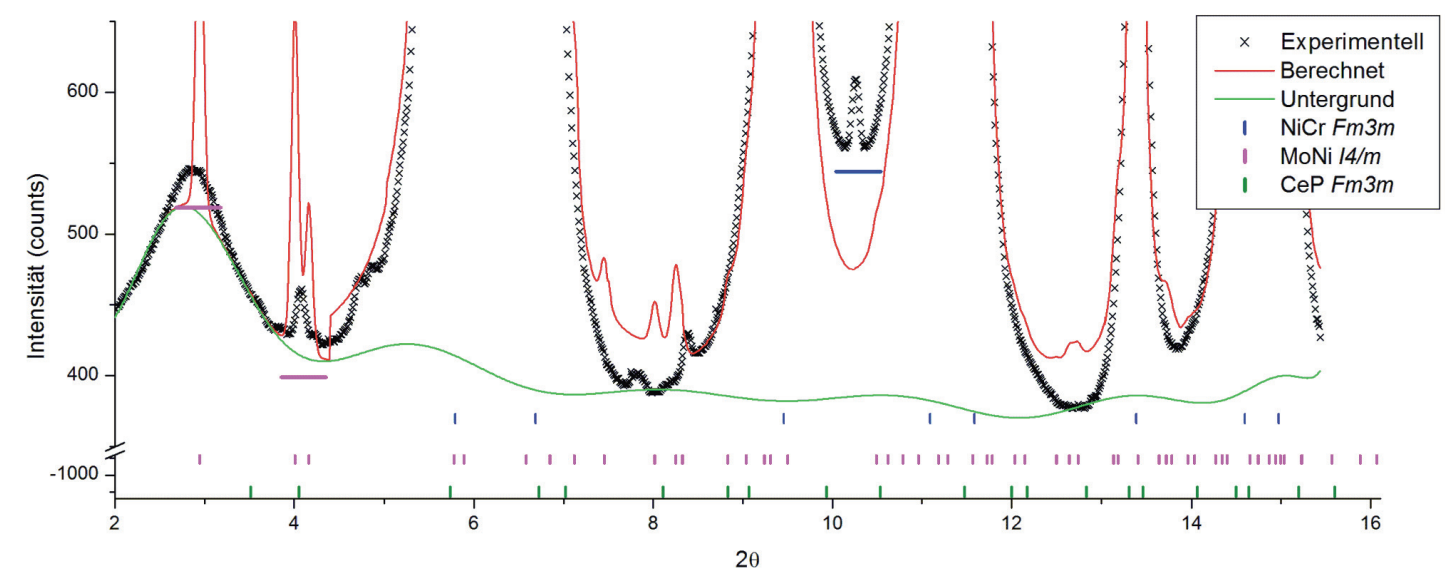

Abb. 51: Röntgendiffraktogramme der Ni-Cr-Mo-Legierung Wirong9. Auswertung mit dem Programm GSAS (PETRA, P02.1; $\lambda=0,2086 \AA$ ).

(a) Gesamtes Röntgendiffraktogramm. Reflexe der Ni-Cr-Hauptphase $(F m \overline{3} m)$ sind indiziert. (b) Ausschnitt mit Reflexen möglicher Ausscheidungsphasen $\mathrm{MoNi}_{4}(I 4 / m, a=5,754(3) \AA$ und $b=3.496(2) \AA$ (verfeinert)) und $\mathrm{CeP}(F m \overline{3} m, a=5,909 \AA$, Vedel et al., 1987). Magentafarben unterstrichene Bereiche weisen auf gute Übereinstimmung der $\mathrm{MoNi}_{4}$-Phase mit beobachteten Reflexen und der blau unterstrichene Bereich auf einen unerklärten Reflex hin. 


\subsubsection{Co-Cr-Mo-Legierung Remanium GM 800+}

Für die quantitative Phasenanalyse erwies sich der Klammermodellguss Remanium GM $800+$ wie auch schon die Ni-Cr-Mo-Legierung Wiron99 als zu grobkörnig (Abb. 50b). Daher wurden ebenfalls Messungen an der pulverisierten Probe (in Glaskapillare) durchgeführt (2dTransmissionsbilder s. Anhang Abb. B.6). Es konnten $\alpha$-MoCr $(\operatorname{Im} \overline{3} m), \epsilon-\mathrm{CoCr}\left(P 6_{3} / m m c\right)$ und $\gamma$-CoCr $(F m \overline{3} m)$ eindeutig als Hauptphasen identifiziert werden (Abb. 52a und b). Die Phasenanteile für die Hauptphasen wurden anhand von Messungen an der Beamline P02.1 und am Bruker Smart Apex II mit dem Programm GSAS und teilweise mit MAUD bestimmt, wobei für die verschiedenen Phasen die Elementgehalte aus den Mikrosondenergebnissen weitgehend übernommen wurden (Anhang Tab. A.11). Die Ergebnisse aus diesen Phasenanalysen waren jedoch nicht eindeutig. Obwohl für alle Rechnungen die gleichen Phasen mit gleicher chemischer Zusammensetzung und gleichen atomaren Auslenkungsparametern verwendet wurden, gab es deutliche Unterschiede in den berechneten Phasenanteilen. Zudem änderten sich die Phasenanteile deutlich, wenn die chemische Zusammensetzung oder die atomaren Auslenkungsparameter verändert wurden. Ein zusätzliches Problem, das in den Berechnungen mit MAUD ersichtlich wurde, war, dass mindestens eine Phase texturiert war (Anhang Abb. B.7). Dadurch wichen die gemessenen Intensitäten von denen einer Probe mit regellos verteilten Kristalliten ab und führten sehr wahrscheinlich zu fehlerbehafteten Ergebnissen, da dieser Textureffekt weder mit GSAS noch mit MAUD sinnvoll korrigiert werden konnte.

Neben den Hauptphasen könnten nach Gupta (2005) weitere Ausscheidungsphasen wie die $\sigma$ oder die R-Phase auftreten ( $\sigma$-Phase: $P 4_{2} / m n m$; R-Phase: $\left.R \overline{3}\right)$, die sich jedoch nicht eindeutig identifizieren lassen. 
a)

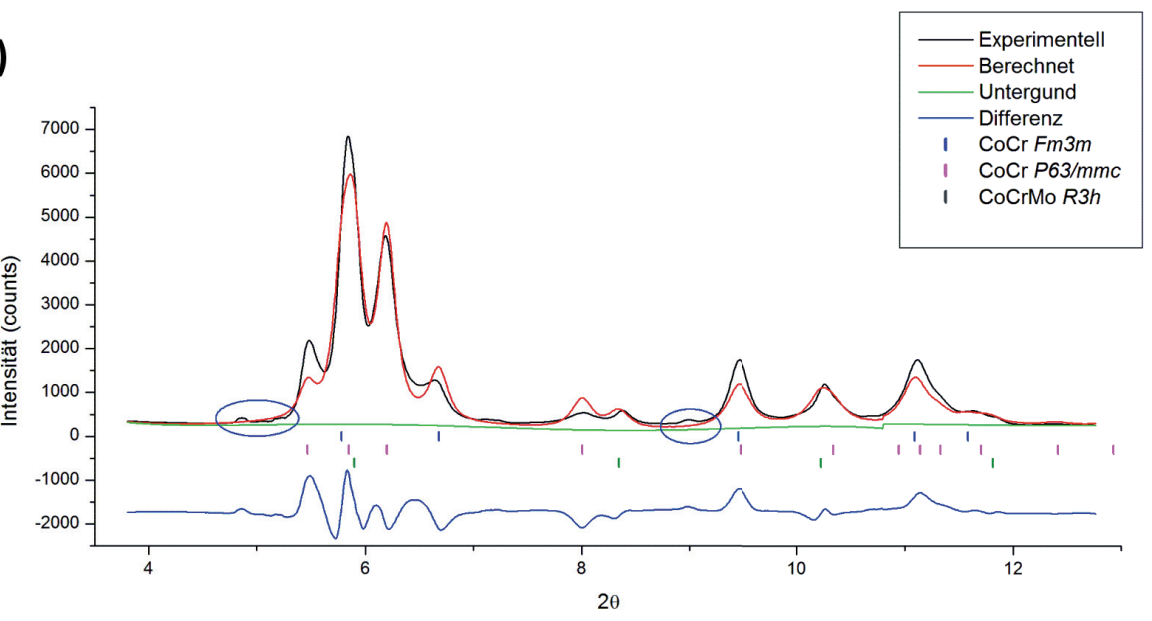

b)

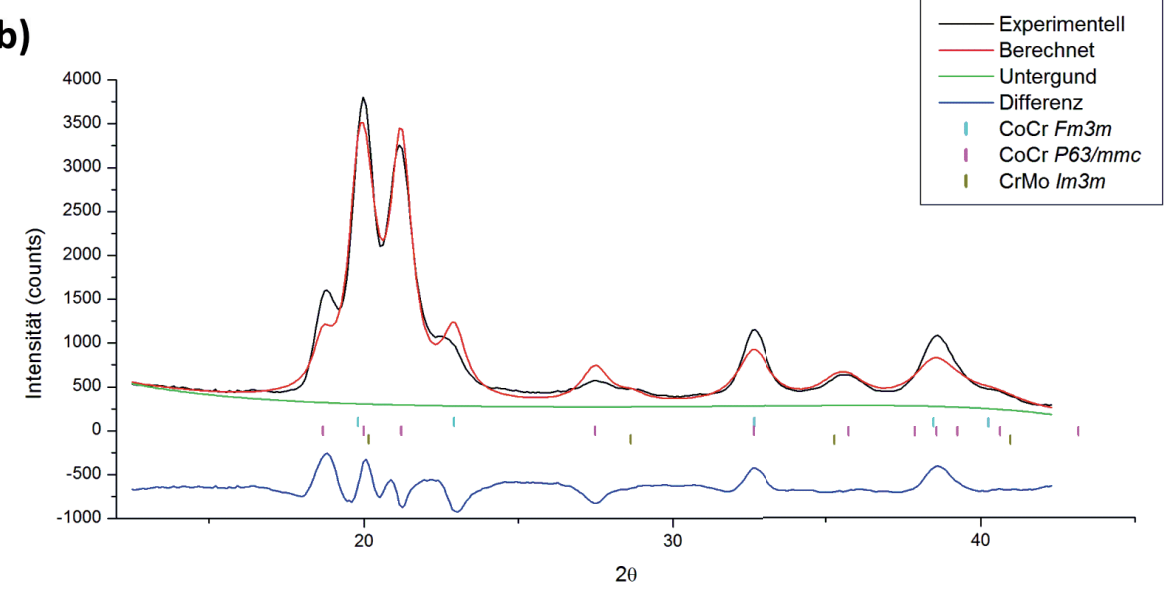

Abb. 52: Röntgendiffraktogramme der Co-Cr-Mo-Legierung Remanium GM 800+. Auswertung mit dem Programm GSAS.

(a) Profilanpassungen für die Hauptphasen $\epsilon-\mathrm{Co}_{0,70} \mathrm{Cr}_{0,26} \mathrm{Mo}_{0,04}, \gamma-\mathrm{Co}_{0,68} \mathrm{Cr}_{0,24} \mathrm{Mo}_{0,08}$ und $\alpha-\mathrm{Cr}_{0,85} \mathrm{Mo}_{0,15}$ (P02.1; PETRA; $\lambda=0,2086 \AA$ ). Eingekreiste Bereiche deuten auf nicht angepasste Reflexe von Ausscheidungsphasen hin.

(b) Profilanpassungen für die Hauptphasen $\epsilon-\mathrm{Co}_{0,70} \mathrm{Cr}_{0,26} \mathrm{Mo}_{0,04}, \gamma-\mathrm{Co}_{0,68} \mathrm{Cr}_{0,24} \mathrm{Mo}_{0,08}$ und $\alpha-\mathrm{Cr}_{0,85} \mathrm{Mo}_{0,15}$ (Bruker Smart Apex II; $\lambda=0,7093 \AA$ ). 


\subsubsection{Füllungen in extrahierten Zähnen}

\subsubsection{Amalgamfüllung}

Die Amalgamfüllung setzte sich aus den Phasen AgSn (Pmmn) zu 48,7(3) Gew.\%, $\mathrm{Ag}_{2} \mathrm{Hg}_{3}$ (I23) zu 41,2(3) Gew.\% und $\mathrm{Cu}_{6} \mathrm{Sn}_{5}\left(P 6_{3} / m m c\right)$ zu 10,2(3) Gew.\% zusammen $\left(R_{w p}=3,57 \%\right)$. Zur Bestimmung der Phasenanteile wurde im Rietveld-Programm GSAS der Untergrund manuell durch Interpolationspunkte unter Verwendung von 10 Polynomialtermen angepasst und alle Gitterparameter, der Nullpunktsfehler sowie die Profilparameter GW und LY freigegeben (s. Abb. 53, Tab. A.7).

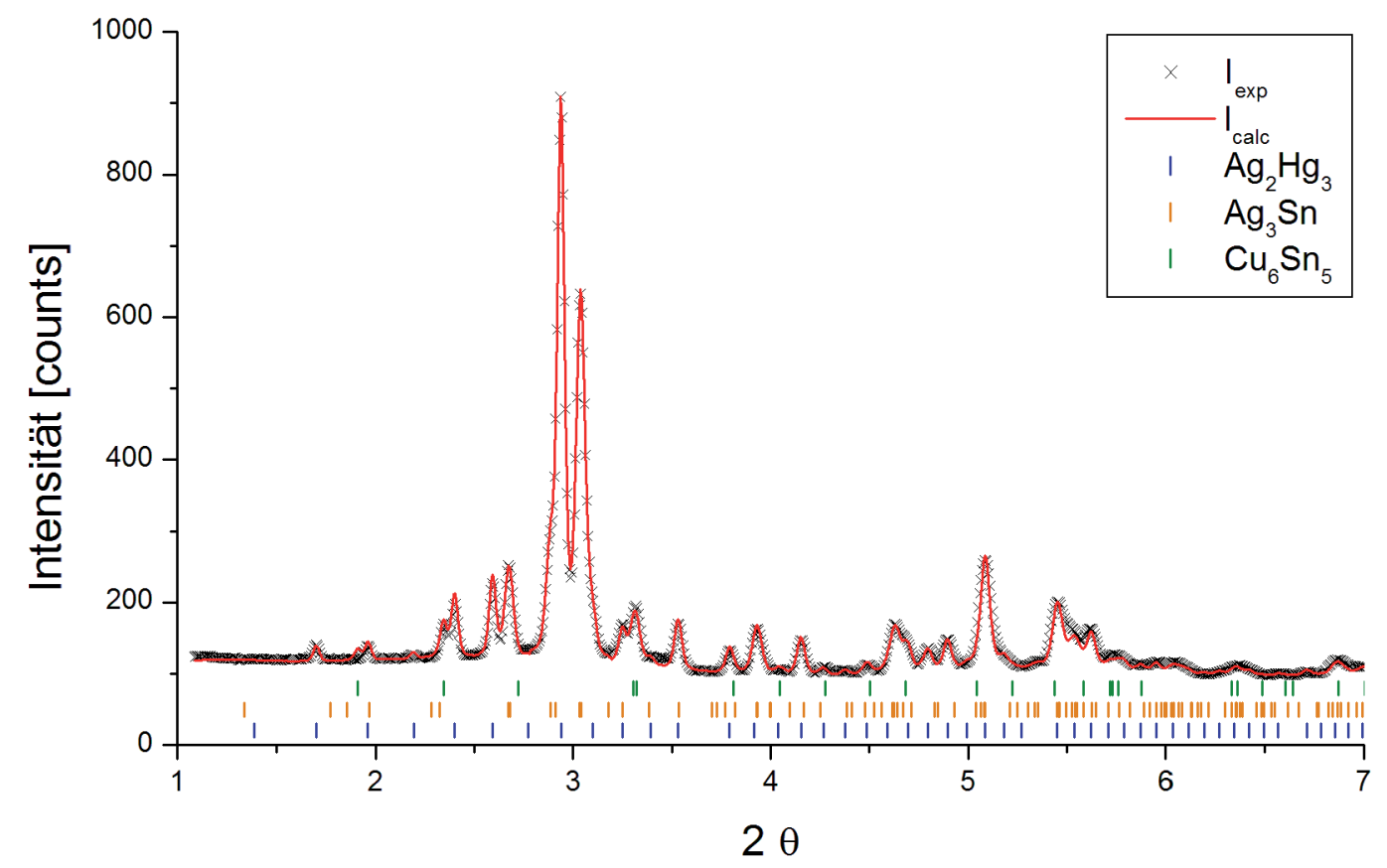

Abb. 53: Röntgendiffraktogramm einer alten Amalgamfüllung. Gemessen an der Beamline BW5 $(\lambda=0,1214 \AA)$; ausgewertet mit dem Rietveld-Programm GSAS.

\subsubsection{Goldfüllung mit Unterfüllung}

Die Phasenanalyse der Goldlegierung zeigte neben dem Hydroxylapatit des Zahnschmelzes bzw. Dentins nur eine Au-Phase, $F m \overline{3} m$ mit $a=3,9908(8) \AA\left(R_{w p}=2,92 \%\right.$; Abb. 54a). Der im Vergleich zu reinem Gold $\left(a_{A u}=4,0782 \AA\right.$; Massalski, 1990) stark verringerte Gitterparameter deutet daraufhin, dass dem Gold weitere Metalle wie Pd und Pt $\left(a_{P d}=3,8903 \AA\right.$ und $a_{P t}=3,9236 \AA$; Massalski, 1990) hinzulegiert wurden. Eventuelle Ausscheidungsphasen der Goldlegierung ließen sich bei der vorhandenen 2 $\theta$-Auflösung und dem Untergrund nicht beobachten (Abb. 54a).

Die Phasen der Unterfüllung waren zunächst unbekannt, da in den vergangenen Jahren sehr unterschiedliche Werkstoffe als Unterfüllung verwendet wurden und das Alter der Füllung ebenfalls nicht bekannt war. Röntgenographisch wurden die kristallinen Phasen Hydroxylapatit $\left(\mathrm{Ca}_{5}\left(\mathrm{PO}_{4}\right)_{3}(\mathrm{OH}), P 6_{3} / m\right.$; Saenger \& Kuhs, 1992), Zinkit (ZnO, P6 $6_{3} m c$; Albertsson et al., 1989) 


\begin{tabular}{l|cc}
\hline \hline Phasen & ohne Texturkorrekturfaktor & mit Texturkorrekturfaktor \\
\hline Hydroxylapatit & $59,6(4)$ & $59,5(4)$ \\
Zinkit & $39,5(3)$ & $39,3(3)$ \\
Hopeit & $0,9(3)$ & $1,2(3)$ \\
\hline \hline
\end{tabular}

Tab. 18: Ergebnisse aus der Phasenanalyse für die Goldunterfüllung. Gemessen an der Beamline BW5 $(\lambda=0,1214 \AA)$ ausgewertet mit dem Rietveld-Programm GSAS. Alle Angaben in Gew.\%.

und Hopeit $\left(\left(\mathrm{Zn}_{1-x} \mathrm{Mg}_{x}\right)_{3}\left(\mathrm{PO}_{4}\right)_{2} \cdot 4 \mathrm{H}_{2} \mathrm{O}\right.$ bzw. $\mathrm{Zn}_{2,38} \mathrm{Mg}_{0,62} \mathrm{P}_{2} \mathrm{O}_{12} \mathrm{H}_{8}$, Pnma; Haussühl et al., 1991) nachgewiesen (Abb. 54b). Die Hydroxylapatitphase, die sehr wahrscheinlich dem der Unterfüllung umgebenden Dentin entstammt, scheint leicht texturiert zu sein, da der 002-Reflex ohne Texturkorrekturfaktoren stark überschätzt wird. Daher wurde für die Phasenanalyse die March/Dollase-Korrektur für bevorzugte Orientierungen angewendet (Dollase, 1986). Der Fit verbessert sich deutlich (von $R_{w p}=5,92 \%$ ohne Korrekturfaktor zu $R_{w p}=5,52 \%$ mit MDKorrekturfaktor) und der MD-Wert nimmt bei freier Verfeinerung leicht zu (MD =1,10(1) regellose Textur: $\mathrm{MD}=1,00$ ), wobei sich die Phasenanteile innerhalb der geschätzten Standardbeweichung nicht signifikant unterscheiden (Tab. 18).

Unter Verwendung des Texturkorrekturfaktors und unter der Annahme, dass die HydroxylapatitPhase aus dem Dentin stammt, ergibt sich für die Unterfüllung eine kristalline Zusammensetzung von 97,0(4) Gew\% ZnO und 3,0(4) Gew\% Hopeit (s. Abb. 54b). Diese Phasen deuten daraufhin, dass ein klassisches Zinkphosphat-Zement als Unterfüllung verwendet wurde (z.B. HARVARD CEMENT mit den Hauptkomponenten $\mathrm{ZnO}, \mathrm{MgO}$ (1/10-tel Gew.\% des ZnO) und orthoPhosphorsäure; Angaben zu den chemischen Vebindungen beziehen sich auf die Ausgangsphasen vor der Abbindereaktion). 
a)

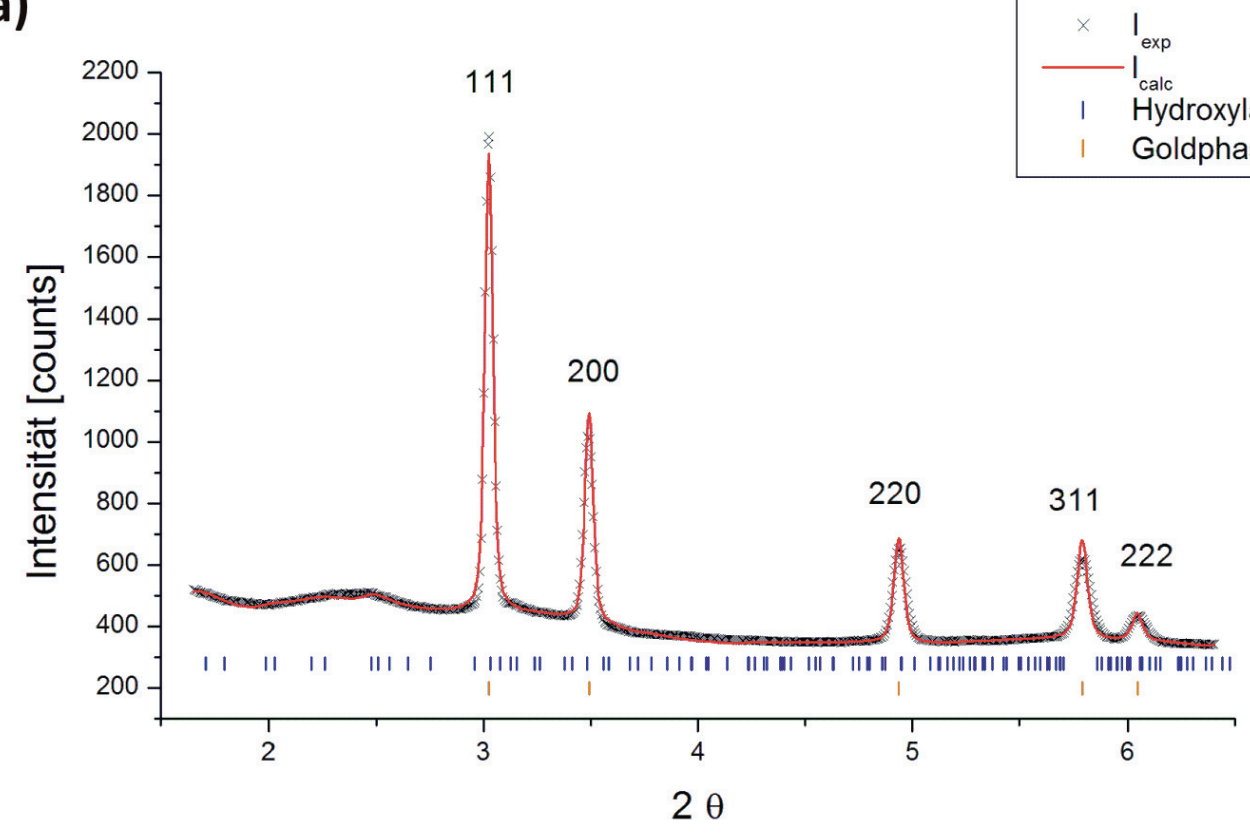

b)

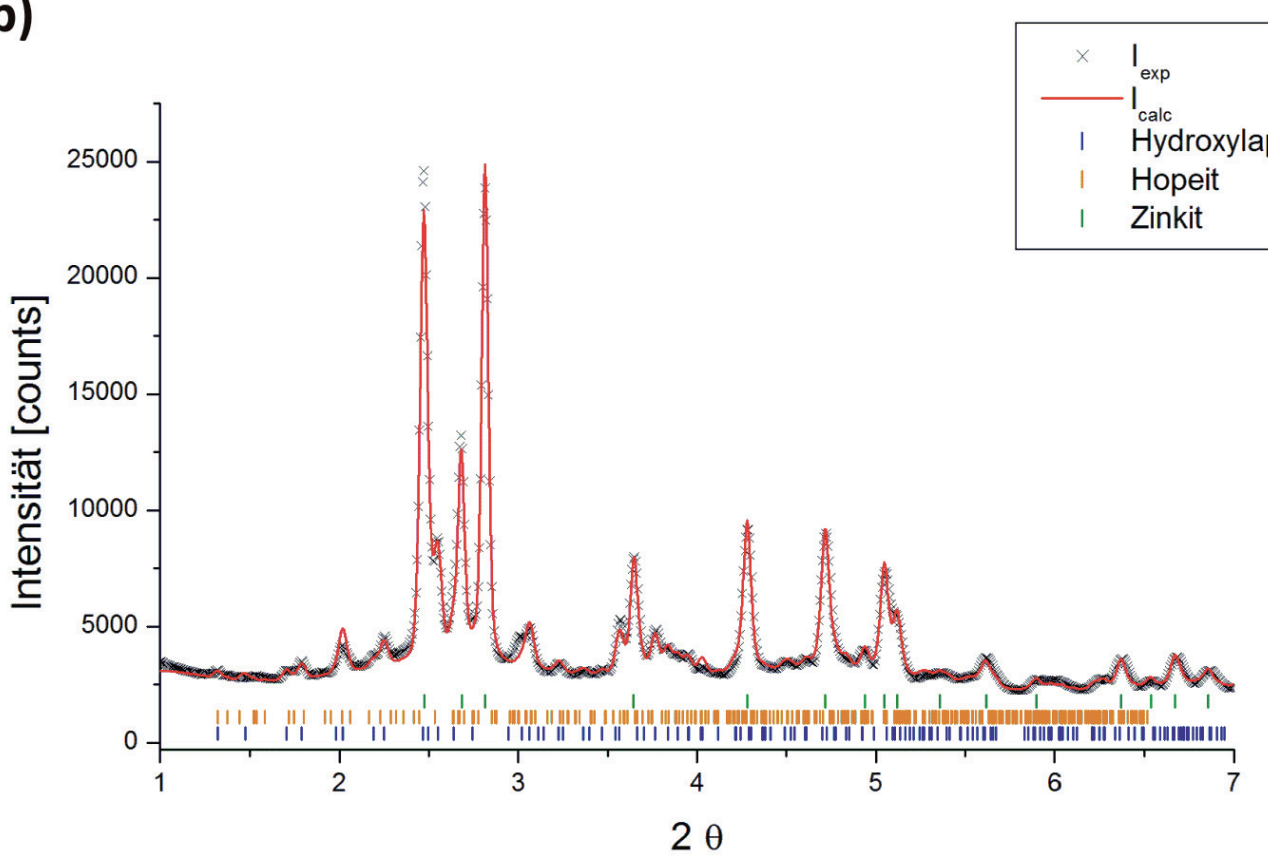

Abb. 54: Röntgendiffraktogramme einer alten Goldfüllung (a) sowie deren ZinkphosphatUnterfüllung (b). Gemessen an der Beamline BW5 $(\lambda=0,1214 \AA)$; ausgewertet mit dem Rietveld-Programm GSAS. Die Reflexe der Goldphase in (a) sind indiziert. 


\subsection{Ergebnisse der röntgenographischen Berechnung von Glasgehalten in Glaskeramiken}

Die Ergebnisse aus den Rietveld-Verfeinerungen der Testserie (A, B und $\mathbf{C})$ von Glas-KristallitPulvermischungen sind in Tab. 19 dargestellt (Bsp. für die Profilanpassungen nach beiden Methoden s. Abb. 55). Daraus geht hervor, dass beide Methoden zur röntgenographischen Bestimmung des Glasanteils geeignet sind, wobei die IS-Methode (Methode mit internem Standard; kurz: ISM) den Glasanteil besser bei geringen Glasgehalten und die Glasmodell-Methode (kurz: GSM) den Glasanteil besser bei größeren Glasgehalten wiedergibt.

Tab. 19: Ergebnisse aus den Rietveld-Verfeinerungen der Glas-Testserie (gemessen am BW5 $(\lambda=\mathbf{0 , 1 2 1 4} \AA)$ und am W2 $(\lambda=\mathbf{0 , 1 2 5 1} \AA))$. Alle Angaben sind in Gew.\%. Fehler der Einwaage: 0,01 Gew.\% (Probenbehälter: Al-Röhrchen).

\begin{tabular}{l|ccc|ccc|ccc} 
& \multicolumn{3}{|c|}{$\mathbf{A}$} & \multicolumn{3}{|c}{$\mathbf{B}$} & \multicolumn{3}{c}{$\mathbf{C}$} \\
& Glas & $\mathrm{CaCO}_{3}$ & $\mathrm{ZnO}$ & Glas & $\mathrm{CaCO}_{3}$ & $\mathrm{ZnO}$ & Glas & $\mathrm{CaCO}_{3}$ & $\mathrm{ZnO}$ \\
\hline Einwaage & 15,0 & 75,0 & 10,0 & 40,3 & 49,8 & 10,0 & 74,9 & 15,1 & 10,0 \\
ISM & $17(2)$ & $73(2)$ & 10,0 & $46,9(2)$ & $43,1(2)$ & 10,0 & $81,7(9)$ & $8,3(9)$ & 10,0 \\
GSM & $10(1)$ & $80,2(1)$ & $10,3(1)$ & $42(3)$ & $48(3)$ & $10,3(6)$ & $77(1)$ & $14,3(1)$ & $9,1(2)$ \\
Mittelwert & $13(1)$ & $76,6(8)$ & $10,1(5)$ & $44,2(3)$ & $46(2)$ & $10,2(3)$ & $79(1)$ & $11(1)$ & $9,6(1)$
\end{tabular}

Die Proben aus der Glastestserie wurden alle in Reinst-Aluminiumröhrchen in Transmission an den Beamlines BW5 und W2 gemessen. Um die Anwendbarkeit auch auf verschiedene Probenbehälter zu testen, wurde eine weitere Testmischung mit ähnlicher Zusammensetzung wie B erstellt (B1; Tab. 20) und sowohl in Reinst-Aluminium-Röhrchen als auch in röntgendurchlässigen PI-Kapillaren (PI: Polyimid) gemessen. Die dafür ausgesuchte PI-Kapillare wies im Röntgenbeugungungsbild keine Reflexe kristalliner Phasen und nur einen breiten Beugungsring geringer Intensität auf (s. Anhang Abb. B.9).

Tab. 20: Ergebnisse aus den Rietveld-Verfeinerungen der Glastestmischung B1 (gemessen am BW5 $(\lambda=0,1214 \AA)$ und am P02.1 $(\lambda=0,2085 \AA))$. Alle Angaben sind in Gew.\%. Fehler der Einwaage: 0,01 Gew.\%.

\begin{tabular}{l|ccc|ccc|ccc} 
& \multicolumn{2}{|c}{ Al-Röhrchen (BW5) } & \multicolumn{3}{c|}{ PI-Kapillare (BW5) } & \multicolumn{3}{c}{ PI-Kapillare (P02.1) } \\
& Glas & $\mathrm{CaCO}_{3}$ & $\mathrm{ZnO}$ & Glas & $\mathrm{CaCO}_{3}$ & $\mathrm{ZnO}$ & Glas & $\mathrm{CaCO}_{3}$ & $\mathrm{ZnO}$ \\
\hline Einwaage & 39,90 & 49,95 & 10,15 & 39,90 & 49,95 & 10,15 & 39,90 & 49,95 & 10,15 \\
ISM & $43,4(8)$ & $46,4(8)$ & 10,15 & $45(4)$ & $45(4)$ & 10,15 & $46,2(1)$ & $43,7(1)$ & 10,15 \\
GSM & $47,9(1)$ & $42,42(6)$ & $9,72(1)$ & $42(2)$ & $48(2)$ & $10,1(3)$ & $47,0(2)$ & $43,79(3)$ & $9,17(1)$
\end{tabular}

Beide Methoden wurden auf die Dentalkeramiken IPS e.max Press, Vita In-Ceram Alumina, Vita In-Ceram Zirkonia und Vitablocs Esthetic Line angewandt. Als Glasmodell kam dabei für alle Proben nicht das Strukturmodell von reinem $\mathrm{SiO}_{2}$-Glas in Frage, da die chemische Zusammensetzung der vorhandenen Gläser zu stark von diesem abwich, was auch im Beugungsbild 
anhand des in $2 \theta$-verschobenen breiten Beugungsrings ersichtlich ist $\left(d_{\text {Carnegieit }}=2,042(2) \AA\right.$; Tab. A.12, Abb. B.11b).

Im Fall der LS2-Keramik IPS e.max Press wurden die kristallinen Phasen $\mathrm{Li}_{2} \mathrm{Si}_{2} \mathrm{O}_{5}, \mathrm{Li}_{3} \mathrm{PO}_{4}$ und je nach Verarbeitungsgrad $\mathrm{Li}_{2} \mathrm{SiO}_{3}$ in die Phasenanalyse einbezogen. Für die Anwendung der Glasmodell-Methode wurde die Glasphase durch die $\mathrm{Li}_{2} \mathrm{Si}_{2} \mathrm{O}_{5}$-Phase mit einer Kristallitgröße von $14 \AA$ simuliert (Ergebnisse s. Tab. 21; Abb. B.10).

Tab. 21: Ergebnisse aus den Rietveld-Verfeinerungen zur Bestimmung der Glasanteile der Dentalkeramik IPS e.max Press vor und nach dem Heißpressen. Angegeben sind die Mittelwerte aus mehreren Einzelmessungen (Beamline BW5; $\lambda=0,1278 \AA$ ), die von der Keramik in PI-Kapillaren und in Al-Röhrchen durchgeführt wurden.

\begin{tabular}{|c|c|c|c|c|c|c|c|c|c|}
\hline Zustand & Methode & $\begin{array}{l}\text { Glas } \\
\text { [Gew.\%] }\end{array}$ & $\begin{array}{l}\mathrm{Li}_{2} \mathrm{Si}_{2} \mathrm{O}_{5} \\
\text { [Gew.\%] }\end{array}$ & $\begin{array}{c}\mathrm{Li}_{3} \mathrm{PO}_{4} \\
{[\mathrm{Gew} . \%]}\end{array}$ & $\begin{array}{l}\mathrm{Li}_{2} \mathrm{SiO}_{3} \\
\text { [Gew.\%] }\end{array}$ & $\begin{array}{c}\mathrm{Li}_{2} \mathrm{ZnSiO}_{4} \\
\text { [Gew.\%] }\end{array}$ & $\begin{array}{l}R_{w p} \\
{[\%]} \\
\end{array}$ & $\mathrm{n}^{1}$ & Behälter \\
\hline \multirow[t]{4}{*}{ Rohling } & ISM & $52,4(1,9)$ & $40,5(1,6)$ & $4,7(0,2)$ & $0,89(0,04)$ & $1,45(5)$ & 9,46 & $3 * 3$ & $\mathrm{PI}$ \\
\hline & & $53,9(1,3)$ & $40,0(1,2)$ & $4,5(0,1)$ & $0,05(0,02)$ & $1,45(4)$ & 12,54 & $3 * 3$ & $\mathrm{Al}$ \\
\hline & GSM & $28,6(0,9)$ & $62,7(0,8)$ & $5,9(0,1)$ & $1,2(0,5)$ & $1,6(0,5)$ & 9,86 & $3^{*} 3$ & PI \\
\hline & & $47,9(0,5)$ & $45,9(0,8)$ & $4,2(0,1)$ & $0,8(0,1)$ & $1,2(0,6)$ & 12,92 & $3^{*} 3$ & $\mathrm{Al}$ \\
\hline \multirow[t]{4}{*}{ Gusskanal } & ISM & $53,4(0,5)$ & $38,5(1,4)$ & $4,3(0,3)$ & $0,81(0,06)$ & $3,0(1,3)$ & 9,60 & $3 * 3$ & $\mathrm{PI}$ \\
\hline & & $47,6(5,9)$ & $43,2(4,8)$ & $4,7(0,5)$ & $0,08(0,04)$ & $4,5(0,6)$ & 13,85 & $3 * 3$ & $\mathrm{Al}$ \\
\hline & GSM & $24,9(2,6)$ & $62,7(2,1)$ & $6,2(0,4)$ & $0,87(0,9)$ & $5,38(0,11)$ & 9,88 & $3 * 3$ & PI \\
\hline & & $42,8(3,1)$ & $46,8(2,8)$ & $5,7(0,5)$ & $0,61(0,22)$ & $4,10(0,04)$ & 13,26 & $3 * 3$ & $\mathrm{Al}$ \\
\hline
\end{tabular}

${ }^{1}$ Zahl der Messungen (3 verschiedene Positionen unter den $\omega$-Winkeln von $-30^{\circ}, 0^{\circ}$ und $+30^{\circ}$ ).

Die Glasphasen der Vita In-Ceram-Keramiken konnten nicht durch eine sinnvolles Glasstrukturmodell simuliert werden (Beispiel-Diffraktogramm mit $\alpha$-Carnegieit-Struktur im Anhang Abb. B.11). Daher wurde bei diesen Keramiken nur die Methode unter Verwendung des internen Standards angewendet (Ergebnisse s. Tab. 22).

Tab. 22: Ergebnisse aus den Rietveld-Verfeinerungen zur Bestimmung der Glasanteile der Dentalkeramiken Vita In-Ceram Alumina und Vita In-Ceram Zirkonia. Angegeben sind die Mittelwerte aus mehreren Einzelmessungen (Beamline BW5; $\lambda=0,1278 \AA$ ), die von der Keramik in PI-Kapillaren und in Al-Röhrchen durchgeführt wurden.

\begin{tabular}{lcccccccc}
\hline \hline Keramik & Glas & $\begin{array}{c}\text { Korund } \\
\text { [Gew.\%] }\end{array}$ & $\begin{array}{c}\mathrm{ZrO}_{2} \text {-t } \\
{[\mathrm{Gew} . \%]}\end{array}$ & $\begin{array}{c}\mathrm{ZrO}_{2} \text {-m } \\
{[\mathrm{Gew} . \%]}\end{array}$ & $\begin{array}{c}\mathrm{ZrO}_{2} \text {-gesamt } \\
\text { [Gew.\%] }\end{array}$ & $\begin{array}{c}\text { R-Wert } \\
{[\text { Gew.\%] }}\end{array}$ & $\begin{array}{c}\mathrm{n}^{1} \\
{[\%]}\end{array}$ & Behälter \\
\hline Zirkonia & $32,7(2,4)$ & $46,1(1,7)$ & $11,1(0,4)$ & $10,0(0,5)$ & $21,17(0,4)$ & 11,75 & $3^{*} 3$ & PI-Kapillare \\
& $32,1(0,8)$ & $47,3(0,5)$ & $11,1(0,1)$ & $9,6(0,1)$ & $20,71(0,1)$ & 13,20 & $3 * 3$ & Al-Röhrchen \\
\hline \multirow{2}{*}{ Alumina } & $38,1(2,0)$ & $61,9(2,0)$ & & & & 11,22 & $3 * 3$ & PI-Kapillare \\
& $38,2(2,3)$ & $61,8(2,3)$ & & & & 12,70 & $2 * 3$ & Al-Röhrchen \\
\hline \hline
\end{tabular}

${ }^{1}$ Zahl der Messungen (2-3 verschiedene Positionen unter den $\omega$-Winkeln von $-30^{\circ}, 0^{\circ}$ und $+30^{\circ}$ ).

Im Fall der Keramik Vitablocs Esthetic Line konnten zur Bestimmung des Glasanteils als kristalline Phasen nur die Phasen Nephelin und Sanidin neben dem Zinkit-Standard in die Rietveldverfeinerung eingeschlossen werden, da die 20-Auflösung sowohl am BW5 als auch am P02.1 bei den gewählten Messbedingungen nicht hoch genug war, um weiter zwischen den Feldspatphasen zu unterscheiden (Abb. 56). In die Auswertung gingen die Röntgendiffraktogramme des Feld- 
spatkeramikpulvers mit Zinkitphase in Al-Röhrchen am BW5 und in PI-Kapillaren am P02.1 ein (Tab. 23). Am BW5 wurde auch die in PI-Kapillare gefüllte Probe gemessen, wobei sich jedoch das Untergrund/Peak-Verhältnis zur Ermittlung des Glasanteils dieser Probe als zu hoch herausstellte. Zur Simulation des Glasanteils wurde eine Sanidinphase mit einer Kristallitgröße von $13 \AA$ verwendet.

Tab. 23: Ergebnisse aus den Rietveld-Verfeinerungen zur Bestimmung der Glasanteile der Dentalkeramik Vitablocs Esthetic Line. Gemessen am BW5 $(\lambda=0,1214 \AA)$ in Al-Röhrchen und am P02.1 (PETRA; $\lambda=0,2086 \AA)$ in PI-Kapillaren. Stellvertretend für die Feldspatphasen wurde nur die SanidinPhase in die Rechnung miteinbezogen, da die $2 \theta$-Auflösung niedrig war.

\begin{tabular}{llcccccc}
\hline \hline Methode & Beamline & $\begin{array}{c}\text { Glas } \\
{[\text { Gew.\%] }}\end{array}$ & $\begin{array}{c}\text { Sanidin } \\
{[\text { Gew.\%] }}\end{array}$ & $\begin{array}{c}\text { Nephelin } \\
{[\text { Gew.\%] }}\end{array}$ & $\begin{array}{c}\mathrm{n}^{1} \\
R_{w p} \\
{[\%]}\end{array}$ & Behälter \\
\hline ISM & BW5 & $89,22(0,75)$ & $4,775(0,03)$ & $6,01(0,75)$ & 34 & 9,7 & Al-Röhrchen \\
ISM & P02.1 & $91,31(1,01)$ & $3,85(0,04)$ & $4,83(0,05)$ & 4 & 0,49 & PI-Kapillare \\
\hline GSM $^{2}$ & BW5 & $86,48(0,89)$ & $5,00(0,26)$ & $8,52(0,63)$ & 34 & 10,7 & Al-Röhrchen \\
GSM $^{2}$ & P02.1 & $88,4(9,2)$ & $5,0(0,5)$ & $6,7(0,7)$ & 4 & 0,68 & PI-Kapillare \\
\hline \hline
\end{tabular}


a)

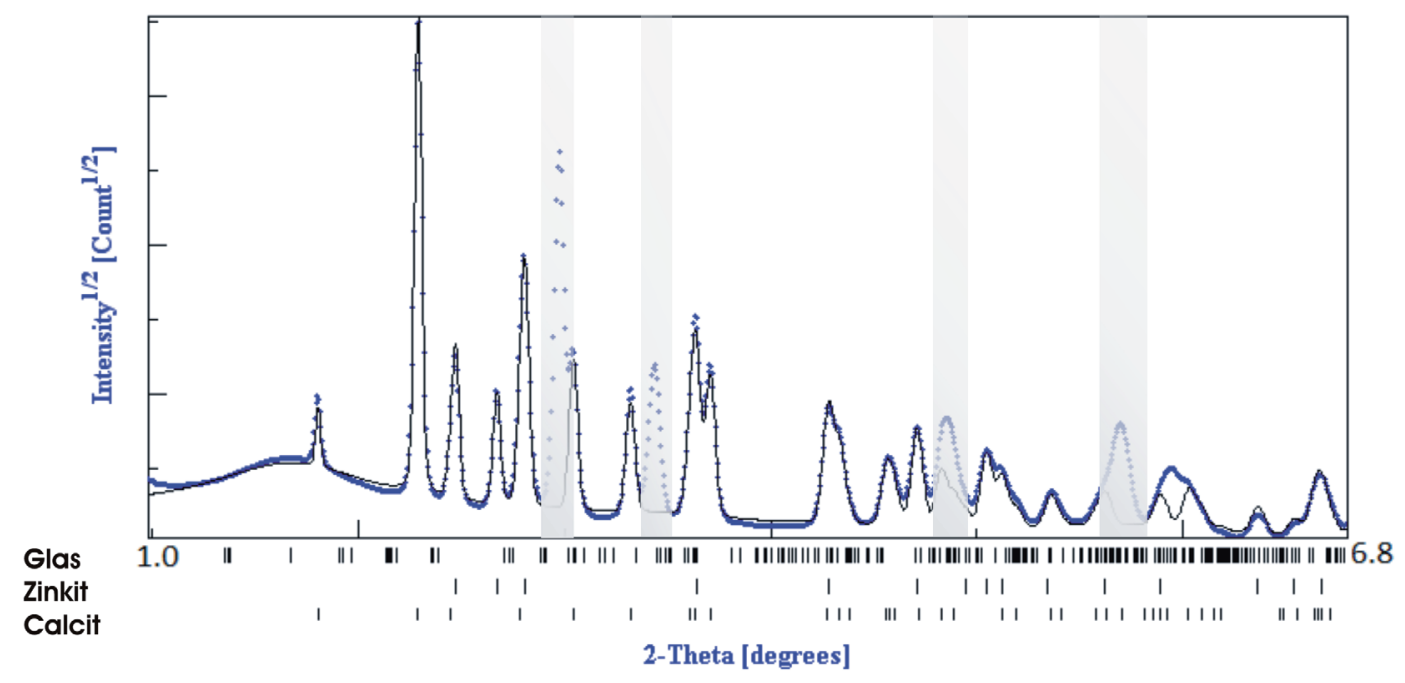

b)

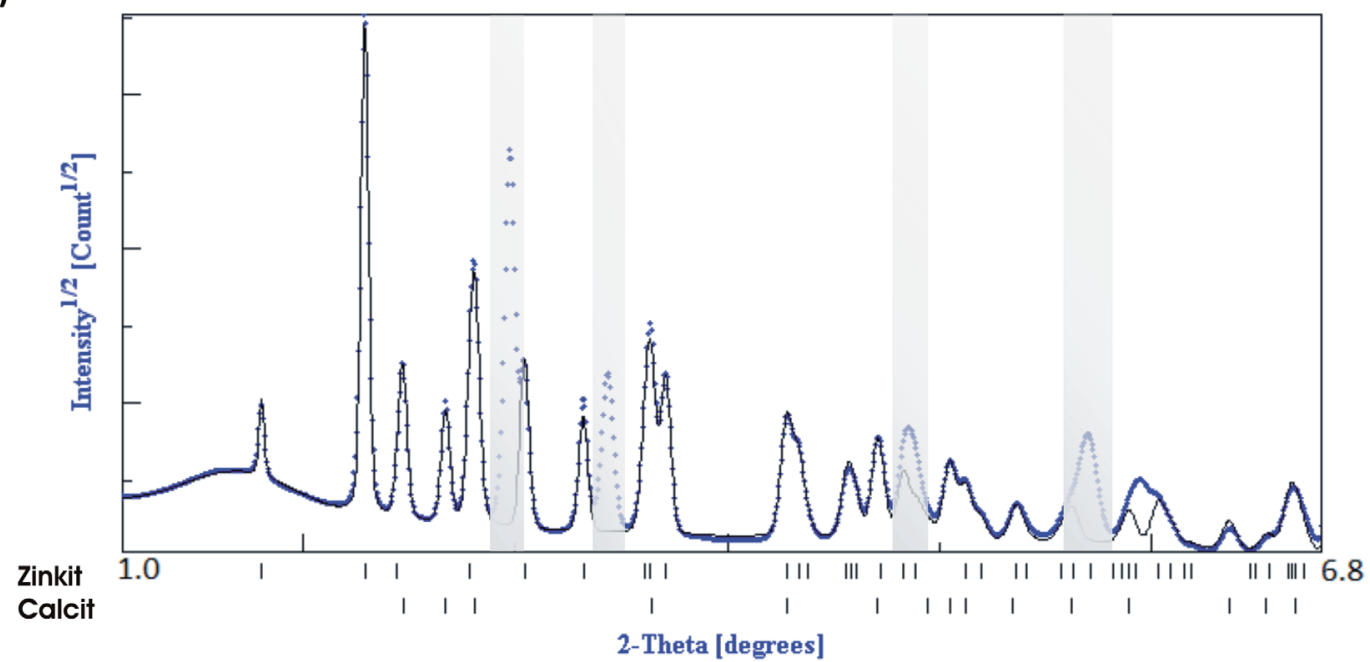

Abb. 55: Röntgendiffraktogramme der Glastestmischung B in Al-Röhrchen. (a) zeigt Profilanpassung nach der GS-Methode ( $\alpha$-Carnegieit mit $8 \AA$ als Kristallitgröße zur Simulation der Glasphase) und (b) nach der IS-Methode (Streuung des amorphen Glases durch breiten Gauss-Peak angepasst). Grau hinterlegte Bereiche markieren Aluminium-Reflexe des Behälters (Al-Röhrchen) und wurden in den Rietveld-Verfeinerungen nicht berücksichtigt (BW5; $\lambda$ $=0,1214 \AA)$. 
a)

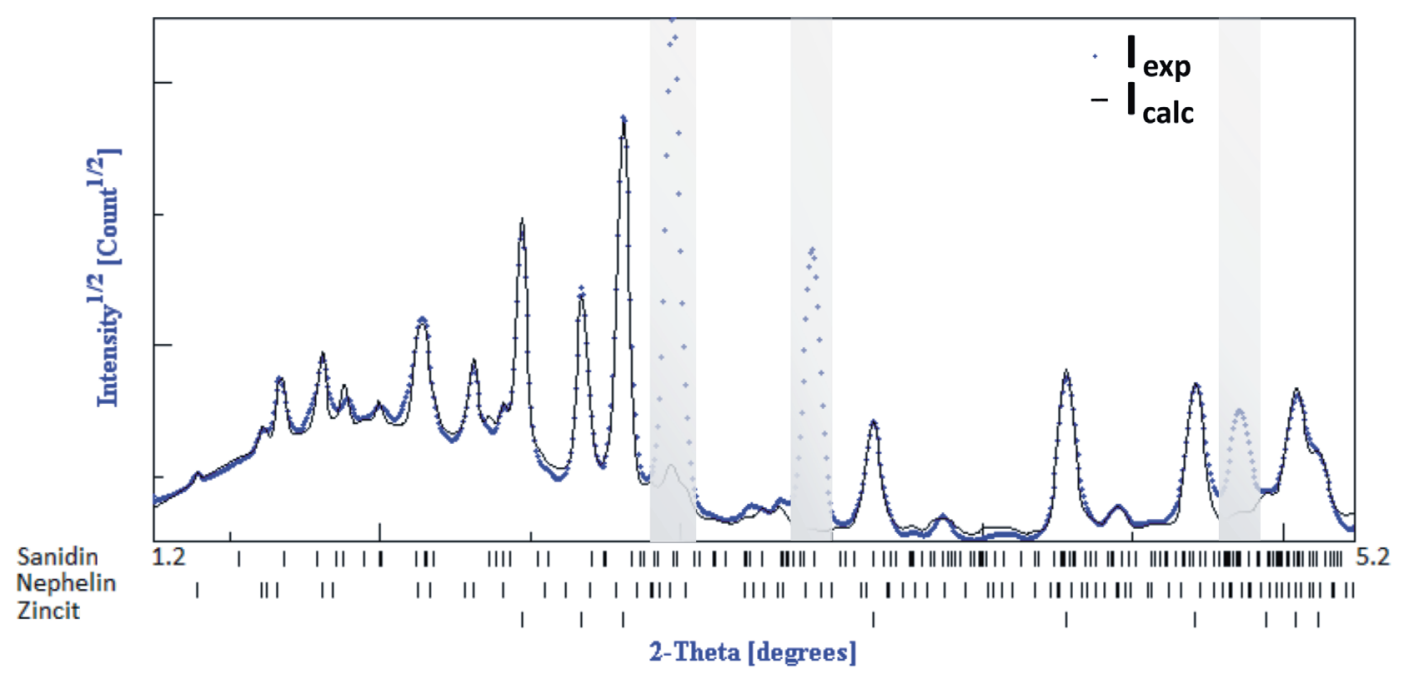

b)

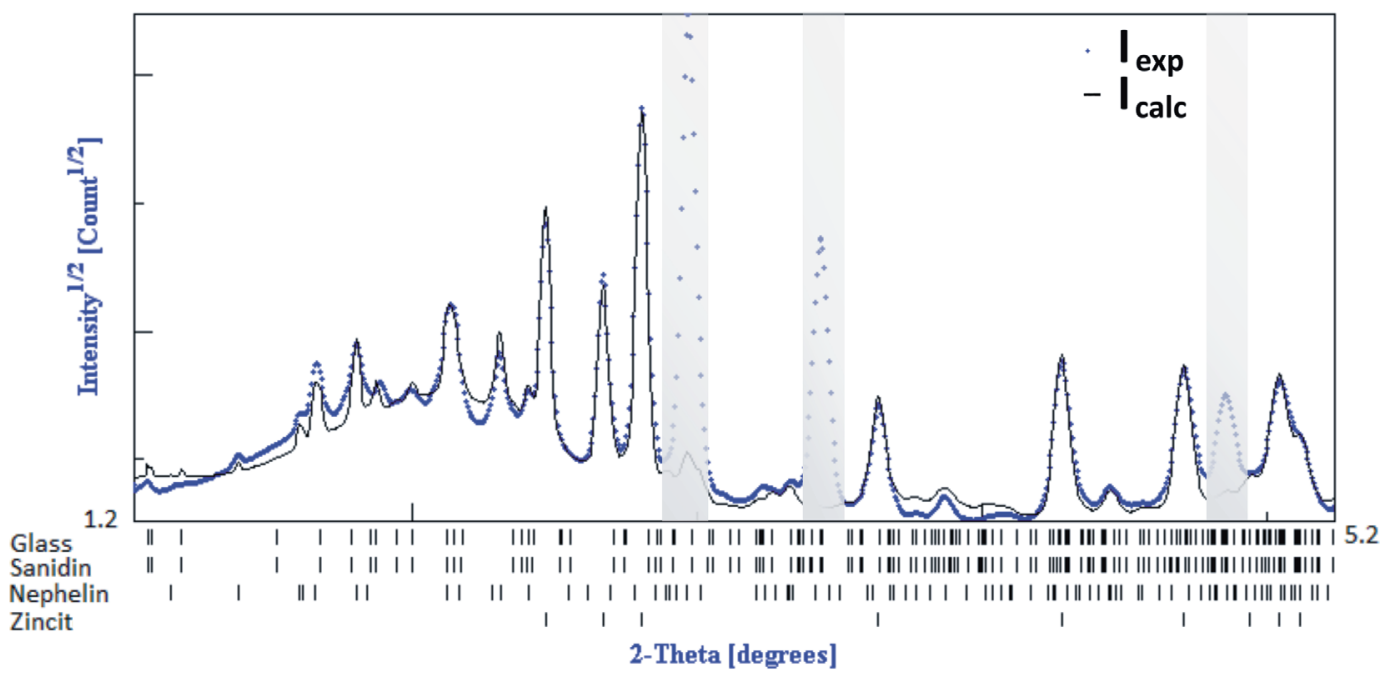

Abb. 56: Röntgendiffraktogramme der Keramik Vitablocs Esthetic Line (Pulver mit ZnO) in Alu-Röhrchen. (a) zeigt Profilanpassung nach der IS-Methode (Streuung des amorphen Glases durch breiten Gauss-Peak angepasst) und (b) nach der GS-Methode (Sanidin mit $13 \AA$ als Kristallitgröße zur Simulation der Glasphase). Grau hinterlegte Bereiche markieren Aluminium-Reflexe des Behälters (Al-Röhrchen) und wurden in den Rietveld-Verfeinerungen nicht berücksichtigt (BW5; $\lambda=0,1214 \AA$ ). 


\subsection{Ergebnisse der Texturanalyse}

\subsubsection{Dentalamalgame Amalcap regular und fast}

In den Synchrotron-Transmissionsaufnahmen beider Dentalamalgame (Anhang Abb. B.12) waren keine eindeutigen Textureffekte zu erkennen. An der Probe Amalcap regular wurde - stellvertretend für beide Dentalamalgame - Texturuntersuchungen sowohl anhand von konventionell gemessenen Polfiguren als auch an Synchrotron-Transmissionsaufnahmen vorgenommen. Allerdings war die Auswertung der konventionell gemessenen Polfiguren nach Bunge (1993) nicht möglich, da aufgrund der Kristallstruktur der Hauptphase $\mathrm{Ag}_{2} \mathrm{Hg}_{3}$ (RG: I23, $a \approx 10,05 \AA$ ) und den vorhandenen Nebenphasen nur eine einzige, nicht überlappende Polfigur (Reflex 222) mit genügend hoher Intensität gemessen werden konnte.

An der Synchrotron-Beamline BW5 wurden an einer $\approx 350 \mu \mathrm{m}$ dicken Scheibe der AmalgamProbe Amalcap regular Transmissionsaufnahmen von $-80^{\circ} \leq \omega \leq+80^{\circ}$ in $4^{\circ}$-Schritten aufgenommen, wobei wegen des hohen Absorptionsvermögens der Amalgamphasen und der Datenqualität nur ein Winkelbereich von $-72^{\circ}$ bis $+56^{\circ}$ genutzt werden konnte. Die Auswertung nach der E-WIMV-Methode in MAUD ergab sowohl für die Hauptphase $\mathrm{Ag}_{2} \mathrm{Hg}_{3}$ (1,0 0 , 3fach regellos in ODF) als auch für die Nebenphasen $\mathrm{Ag}_{3} \mathrm{Sn}$ und $\mathrm{Cu}_{6} \mathrm{Sn}_{5}$ eine regellose Textur (jeweils $1,0 \pm 0,5$ fach regellos in $\mathrm{ODF})$.

\subsubsection{Dentalkomposite, Prothesenkunststoffe und Glasionomerzement}

Mit Ausnahme der Tetric-Serie bestanden die meisten der Dentalkomposite sowie alle Prothesenkunststoffe und der Glasionomerzement nur aus amorphen Komponenten wie Glasfüllpartikel und Polymerisat. Allerdings können auch auf den ersten Blick amorph erscheinende Kunststoffe teilkristalline, texturierte Bereiche enthalten, die sich in einem 2D-Beugungsbild durch Intensitätsmaxima auf den breiten Beugungsringen der Kunststoffe bemerkbar machen. Die vorliegenden Komposite (Ausnahme: die Tetric-Serie mit kristalliner $\mathrm{YbF}_{3}$-Phase) wiesen jedoch alle breite Beugungsringe mit gleichmäßiger Intensitätsverteilung auf (Bsp. s. Anhang Abb. B.14), sodass davon auszugehen ist, dass die Kunststoffkomponenten weder teilkristallin sind noch eine bevorzugte Orientierung gebildet haben.

Auch die Transmissionsaufnahmen der Tetric-Komposite zeigten keine Intensiätsmaxima auf den Beugungsringen der kristallinen $\mathrm{YbF}_{3}$-Komponente, die auf eine Textur hinweisen könnten (Anhang Abb. B.13). Zur Überprüfung wurde stellvertretend an dem Komposit Tetric eine Texturanalyse sowohl mit Nutzung hochenergetischer Synchrotronstrahlung (BW5; $\lambda=0,1240 \AA$ ) als auch mit Nutzung konventioneller Strahlung (XRD 3000 PTS; $\left.\lambda_{C o(K \alpha)}=1,7903 \AA\right)$ durchgeführt.

Für die Auswertung nach der konventionellen Methode wurden Polfiguren von den Reflexen 020, 111, 210, 121, 131 und 101 aufgenommen. Die weitere Auswertung nach Bunge (1993) mit dem DOS-Programm ORTODF ergab jedoch keine eindeutige Lösung, da der Fehler der Polfiguren und der mittlere, absolute C-Koeffizient mit zunehmendem Reihengrad $l$ nicht konvergierten. Die Auswertung der Synchrotronaufnahmen nach der E-WIMV-Methode mit dem Programm MAUD 
konnte erfolgreich durchgeführt werden, wobei sich eine regellose Anordnung der $\mathrm{YbF}_{3}$-Kristallite feststellen ließ $(1,0 \pm 0,3$ fach regellos in ODF $)$.

\subsubsection{Zinksulfatzemente}

Von den Zinksulfatzementen wurde als Beispiel das Zement Detaferm der Firma Detax auf bevorzugte Orientierungen untersucht. Aufgrund der vielen Reflexüberlappungen der vorhandenen Phasen war nur eine mit der Rietveld-Methode kombinierte Texturanalyse sinnvoll. Im 2d-Bild deuteten Intensitätsmaxima auf den (in Abb. 57 geradlinig dargestellten) Beugungsringen auf leichte Vorzugsorientierungen der meisten Phasen hin. Allerdings war eine korrekte, quantitative Texturanalyse wegen folgender Faktoren sowohl nach der konventionellen Methode als auch nach der E-WIMV-Methode nicht möglich:

- (starke) Reflexüberlappungen von 4 verschiedenen Phasen, die zudem oft niedrig symmetrische Kristallsymmetrie besitzen (monoklin, triklin)

- geringe Reflexintensitäten besonders der niedrig symmetrischen Phasen (Gips, ZinksulfatPentahydrat)

- „Schatten“ des Beamstops wurde als Textureffekt interpretiert (Abb. 57)

- Grobkörnigkeit der Bassanit-Phase (Abb. 57)

- nur kleiner Winkelbereich $-75^{\circ} \leq \omega \leq+55^{\circ}$ wurde abgedeckt (fehlende Winkelbereiche können bei der E-WIMV-Methode zu einer falschen Interpretation führen)

a)

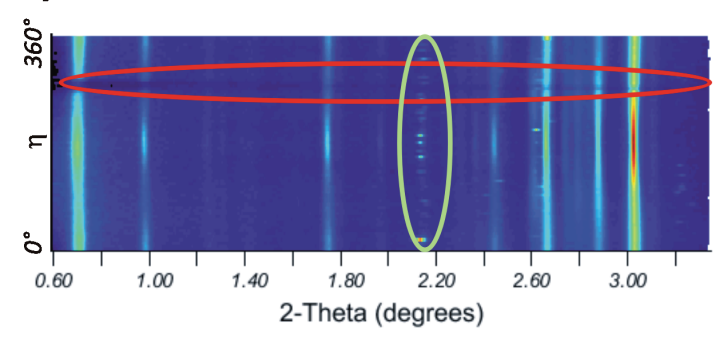

b)

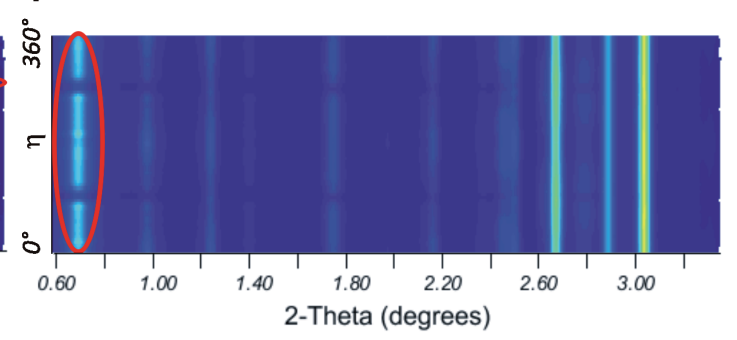

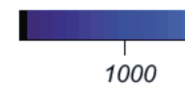

1000

2000

3000

Intensität

Abb. 57: Experimentelle (a) und berechnete (b) 2d-Beugungsbilder des Zinksulfatzements Detaferm (10 Min. Reaktionszeit in Leitungswasser +2 Wochen Lagerung; Texturrechnung nach der E-WIMV-Methode für die Phasen Bassanit, Zinkit und Zinksulfatpentahydrat in $\mathbf{1 0}^{\circ}$-Auflösung). Rot eingekreiste Bereiche weisen auf geringe Intensitäten aufgrund des Beamstops (a) bzw. daraus nicht korrekt umgesetzte Texturberechnungen hin (Phase: Zinksulfatpentahydrat) (b) - grün eingekreister Bereich in (a) zeigt einzelne Reflexe großer Körner der Bassanit-Phase. 


\subsection{4 $\mathrm{Y}-\mathrm{ZrO}_{2}-$ Keramik Cercon base}

Sowohl im endgesinterten als auch im verblendeten Zustand wies die tetragonale $\mathrm{Y}^{-} \mathrm{ZrO}_{2}-$ Phase $\left(P 4_{2} / n m c\right)$ dieser Keramik eine regellose Textur auf (maximal 1,2fach regellos; E-WIMVMethode in $5^{\circ}$-Auflösung; 2d-Transmissionsbild s. Anhang Abb. B.15).

\subsection{5 $\mathrm{Li}_{2} \mathrm{Si}_{2} \mathrm{O}_{5}$-Keramik IPS e.max Press}

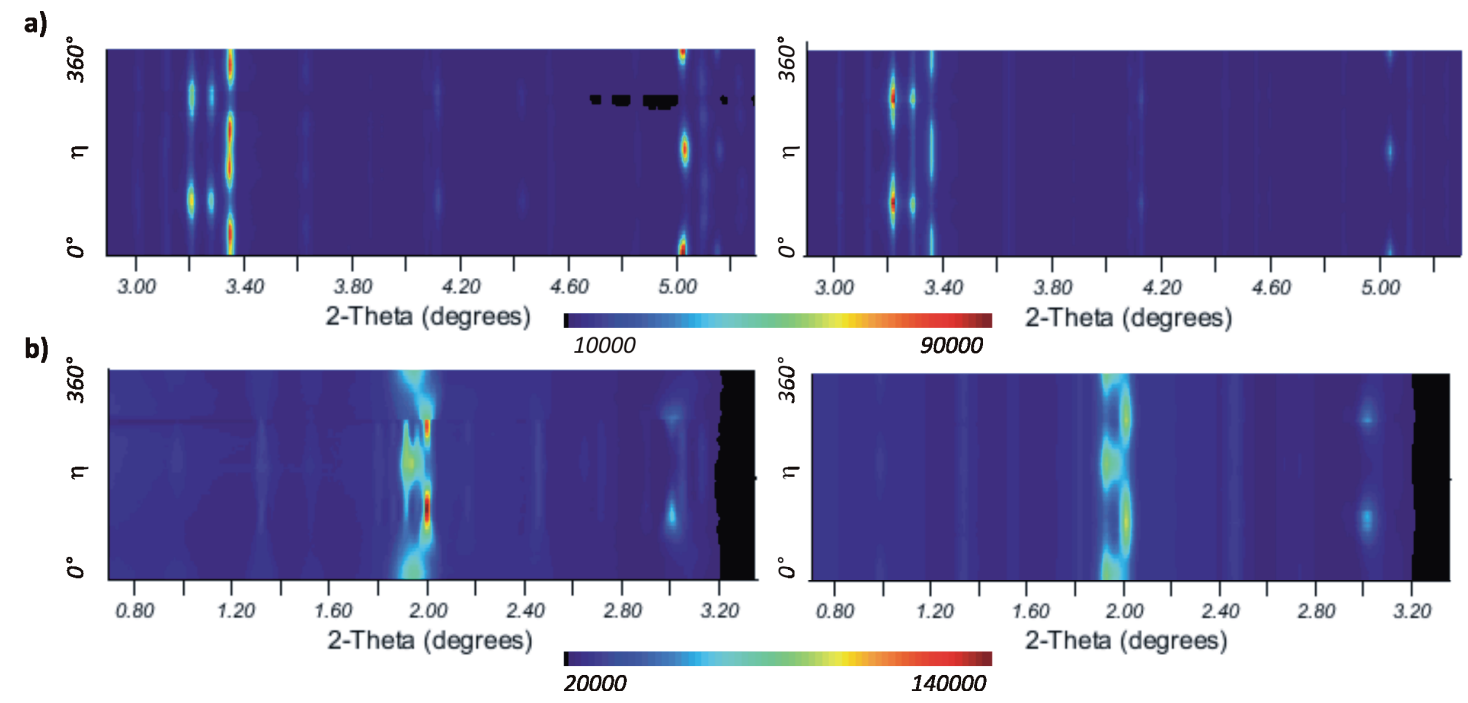

Abb. 58: Beispiele für die kombinierte Textur-Rietveld-Analyse der Keramik IPS e.max Press (a) am Gusskanal (P02.1, $\lambda=0,2088 \AA$ ) und (b) an einer fertigen Keramikscheibe (BW5, $\lambda=\mathbf{0 , 1 2 5 6 1 \AA}$ ). Links: Original-Datensätze; rechts: angepasste Röntgenprofile (Textureinfluss auf die Intensitätsverteilung für die Phasen $\mathrm{Li}_{2} \mathrm{Si}_{2} \mathrm{O}_{5}$ und $\mathrm{Li}_{3} \mathrm{PO}_{4}$ nach der E-WIMV-Methode in $5^{\circ}$-Auflösung berechnet).

Die $\mathrm{Li}_{2} \mathrm{Si}_{2} \mathrm{O}_{5}$-Keramikrohlinge, die in Transmissionsaufnahmen keine bevorzugte Kristallitorientierungen aufwiesen, wurden erhitzt und über Gusskanäle in die gewünschte Form (hier: Scheibe) gepresst. Dabei wurde der Rohling nur erweicht und nicht vollständig geschmolzen. Ein Gusskanal (Dicke $d=$ ca. $2 \mathrm{~mm}$ ) und eine Keramikscheibe (Durchmesser $\varnothing=1,1 \mathrm{~mm}$, Dicke $d=1 \mathrm{~mm}$ ), die im Beugungsbild deutliche Textureffekte zeigten (Abb. 58), wurden mittels Synchrotron-Transmissionsaufnahmen und der in MAUD implementierten E-WIMV-Methode auf Textur untersucht. Konventionelle Texturuntersuchungen waren aufgrund der niedrigen Kristallsymmetrie und der vielen Reflexüberlagerungen nicht möglich. Die Texturen der Phasen $\mathrm{Li}_{2} \mathrm{Si}_{2} \mathrm{O}_{5}$ (monoklin) und $\mathrm{Li}_{3} \mathrm{PO}_{4}$ (orthorhombisch) wurden nach der in MAUD implementierten E-WIMV-Methode in $5^{\circ}$ Auflösung ohne Vorgabe der Probensymmetrie für jeweils einen Messpunkt an einem Gusskanal bzw. an einer fertigen Scheibe berechnet. Ein Vergleich der experimentellen mit den berechneten Polfiguren (Abb. 59 und Abb. 60) zeigt eine gute Übereinstimmung in der Intensitätsverteilung, wobei - im Fall des Gusskanals - insgesamt höhere Intensitätswerte bei den experimentellen Polfiguren erreicht werden. Dieser Unterschied kann dadurch erklärt werden, dass die experimentellen Polfiguren sich auf einzelne Reflexe beziehen, während die zurückberechneten Polfiguren aufein- 
ander normiert sind. Zudem fällt auf, dass die Texturkomponenten von Scheibe und Gusskanal sehr ähnlich sind und dass man die Polfiguren durch eine $90^{\circ}$-Wälzung ineinander überführen kann.

Um die weitere Analyse mit BEARTEX zu erleichtern, wurde testweise auch eine Texturrechnung der $\mathrm{Li}_{2} \mathrm{Si}_{2} \mathrm{O}_{5}$-Phase unter Annahme der pseudo-orthorhombischen Kristallstruktur ( $R G$ : $C c c 2$; Jong et al., 1994) durchgeführt. Dieses Strukturmodell erwies sich jedoch als das unpassendere Strukturmodell, was sich deutlich an der schlechteren Profilanpassung und an dem schlechteren Gütewert bemerkbar machte $\left(R_{w p}\left(\mathrm{Li}_{2} \mathrm{Si}_{2} \mathrm{O}_{5}\right.\right.$ mit $\left.C c c 2\right)=12,84 \%$ versus $R_{w p}\left(\mathrm{Li}_{2} \mathrm{Si}_{2} \mathrm{O}_{5}\right.$ mit $\left.C c\right)$ $=9,94 \%)$.

a)

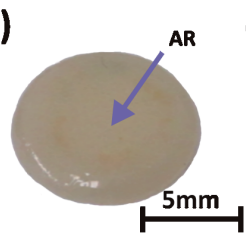

b)

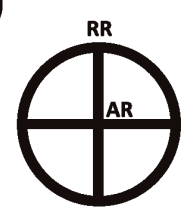

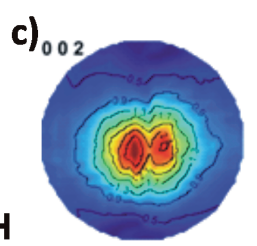

d)

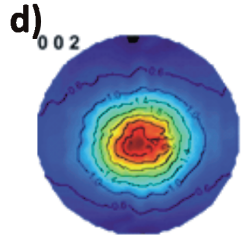

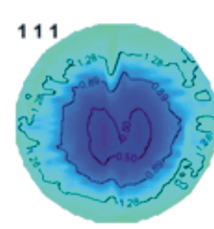
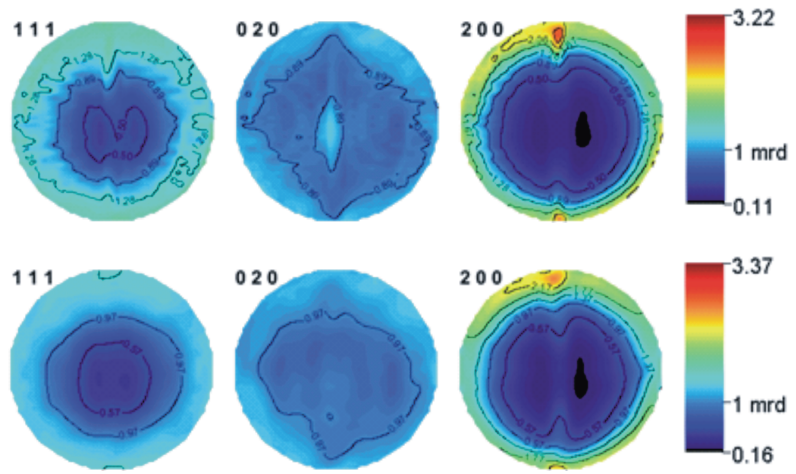

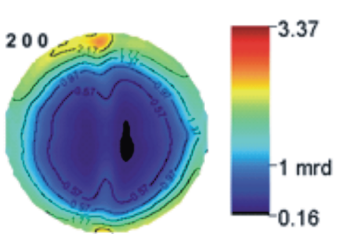

Abb. 59: Experimentelle (c) und nach der E-WIMV-Methode in $5^{\circ}$-Auflösung berechnete (d) Polfiguren der $\mathrm{Li}_{2} \mathrm{Si}_{2} \mathrm{O}_{5}$-Phase für eine fertige, heiß gepresste Scheibe der Keramik IPS e.max Press. In (a) ist der Messpunkt dargestellt und in (b) die Probenrichtungen innerhalb der stereographischen Projektion. Die Abkürzungen AR und RR stehen für Axial- und Radialrichtung.

In den ODFs der monoklin gerechneten $\mathrm{Li}_{2} \mathrm{Si}_{2} \mathrm{O}_{5}$-Phase wurden maximale Intensitäten von 10,56fach (Scheibe) und 8,44fach (Gusskanal) erreicht (Abb. 61 und Abb. 62), während die $\mathrm{Li}_{3} \mathrm{PO}_{4}$-Phase eine verhältnismäßig schwache Textur aufwies $(2,65 f a c h$ in der ODF von der Scheibe und 2,58fach in der ODF vom Gusskanal). Die Kristallite der $\mathrm{Li}_{2} \mathrm{Si}_{2} \mathrm{O}_{5}$-Phase in der Scheibe wiesen eine (001)-Fasertextur parallel zur $\phi_{1}$-Achse bei $\phi_{2}=\Phi=0^{\circ}$ auf, während sie im Gusskanal eine $\Phi$-Faser bei $\phi_{1}=\phi_{2}=90^{\circ}$ bildeten (Abb. 62). 
a)
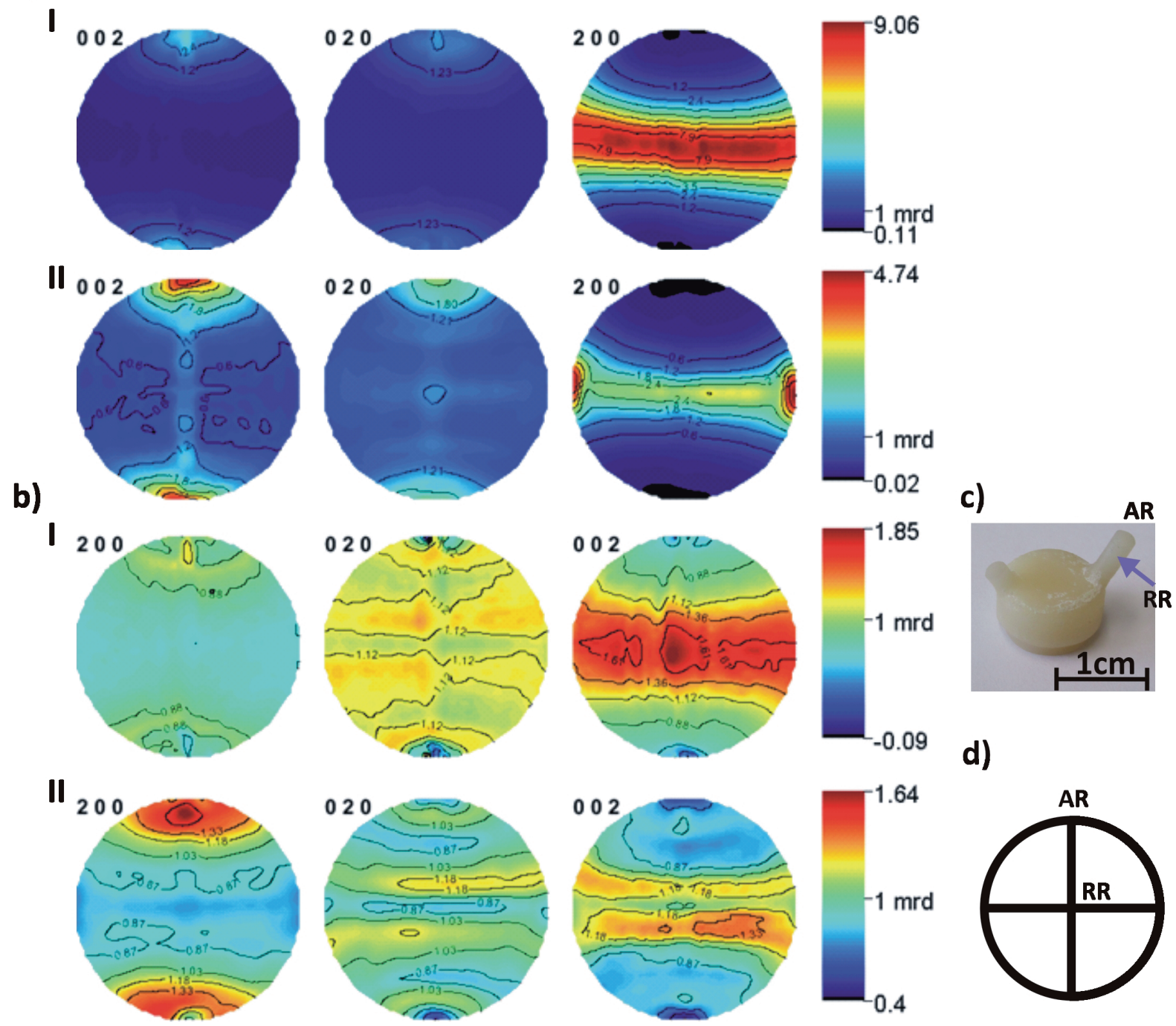

\section{d)}

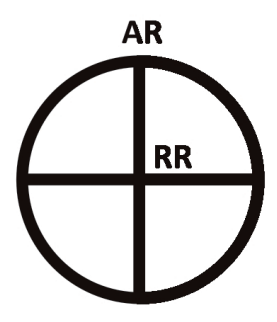

Abb. 60: Gusskanal der LS2-Keramik IPS e.max Press: Experimentelle Polfiguren (I) und nach der E-WIMV-Methode in $5^{\circ}$-Auflösung berechnete Polfiguren (II) der $\mathbf{L i}_{2} \mathbf{S i}_{2} \mathrm{O}_{5}$-Phase (a) sowie der $\mathbf{L i}_{3} \mathbf{P O}_{4}$-Phase (b). In (c) ist der Messpunkt dargestellt und in (d) die Probenrichtungen (Axialrichtung AR und Radialrichtung RR) innerhalb der stereographischen Projektion. 


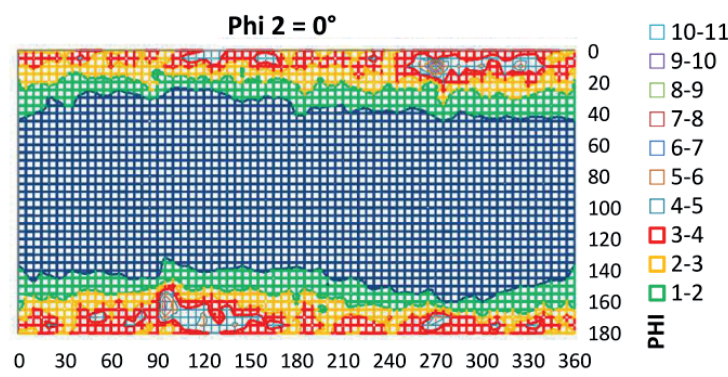

Phi 1

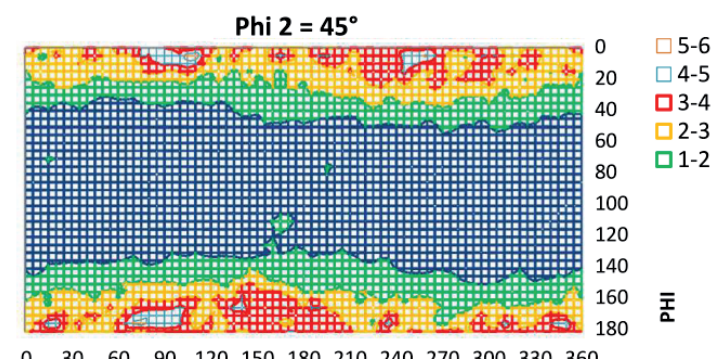

$\begin{array}{lllllllllllll}0 & 30 & 60 & 90 & 120 & 150 & 180 & 210 & 240 & 270 & 300 & 330 & 360\end{array}$

Phi 1

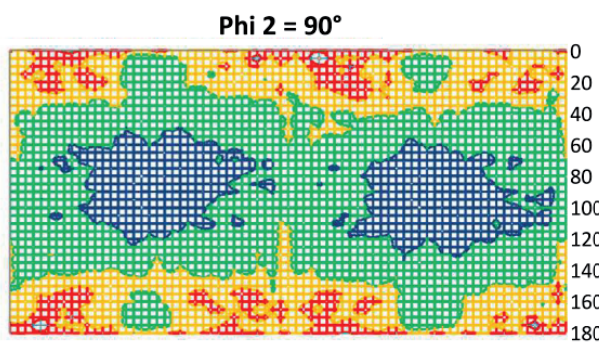

$\square$ 5-6

$\square$ 4-5

$\square 3-4$

$\square 2-3$

1-2

$\square 0-1$

폼

Phi 1

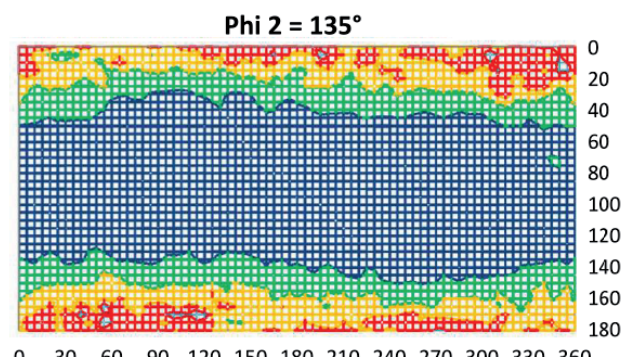

$\square$ 4-5

ㄱ-4

$\square 2-3$

$\square 1-2$

ㅁ-1

$\overline{ }$

Phi 1

Abb. 61: Ausgewählte $\phi_{2}$-Schnitte für die $\mathrm{Li}_{2} \mathrm{Si}_{2} \mathrm{O}_{5}$-Phase der fertigen, heiß gepressten Scheibe der Keramik IPS e.max Press $\left(\phi_{2}=0^{\circ}, 45^{\circ}, 90^{\circ}\right.$ und $\left.135^{\circ}\right)$. Berechnet mit monokliner Kristall- und trikliner Probensymmetrie.
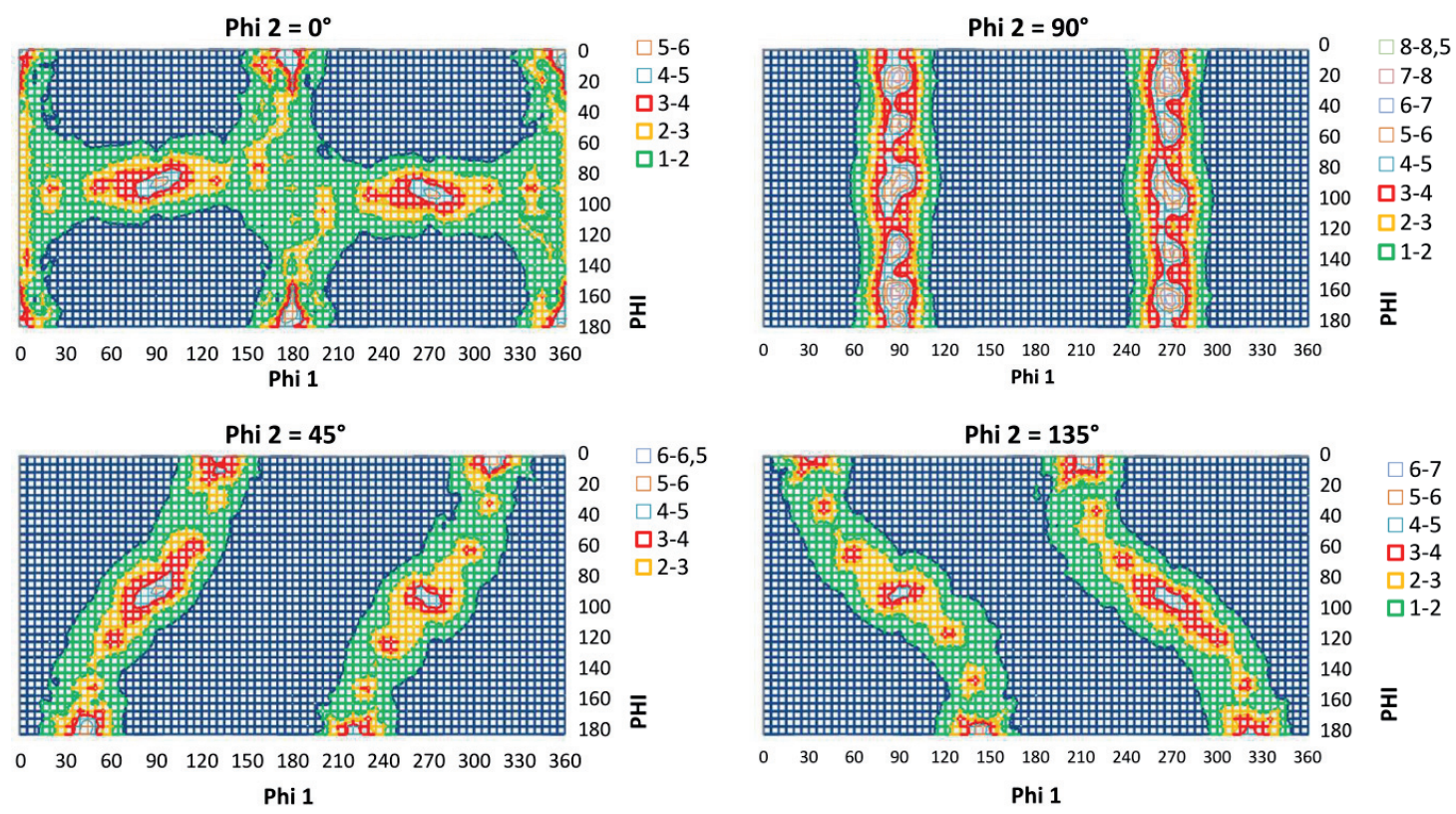

Abb. 62: Ausgewählte $\phi_{2}$-Schnitte für die $\mathrm{Li}_{2} \mathrm{Si}_{2} \mathrm{O}_{5}$-Phase des Gusskanals der Keramik IPS e.max Press $\left(\mathrm{Phi}_{2}=0^{\circ}, 45^{\circ}, 90^{\circ}\right.$ und $\left.135^{\circ}\right)$. Berechnet mit monokliner Kristall- und trikliner Probensymmetrie. 


\subsection{6 $\mathrm{Li}_{2} \mathrm{Si}_{2} \mathrm{O}_{5}$-Keramik IPS e.max CAD}

Die endgesinterte Scheibe des Vollkeramiksystems IPS e.max CAD zeigte in der Texturanalyse mit der E-WIMV-Methode in $5^{\circ}$-Auflösung keine Kristallvorzugsorientierungen (2dTransmissionsbild s. Anhang Abb. B.16). Bei der Texturrechnung erreichte die $\mathrm{Li}_{2} \mathrm{Si}_{2} \mathrm{O}_{5}$-Phase maximal 1,3fach in der ODF.

\subsubsection{Feldspatkeramik Vitablocs - Esthetic Line}

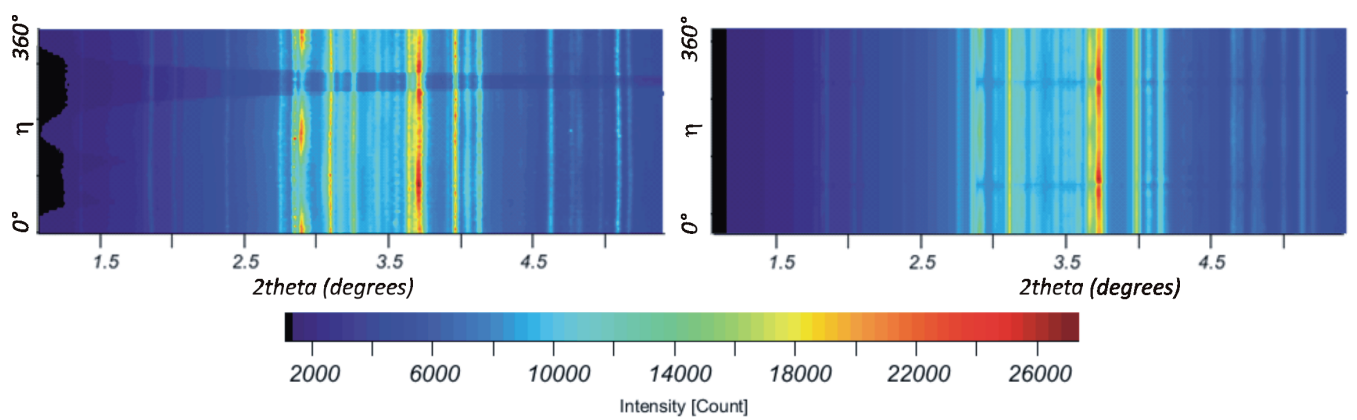

Abb. 63: Beispiel für die kombinierte Textur-Rietveld-Analyse der Keramik Vitablocs Esthetic Line (Messung am CAD/CAM-Block; P02.1, $\lambda=0,2088 \AA$ ). Links: OriginalDatensatz; rechts: angepasstes Röntgenprofil (Textureinfluss auf die Intensitätsverteilung für die Phasen Mikroklin und Sanidin nach der E-WIMV-Methode in $5^{\circ}$-Auflösung berechnet).

In den Synchrotron-Transmissionsaufnahmen am CAD/CAM-Block (Abb. 63) sind deutliche Intensitätsmaxima auf den Beugungsringen zu erkennen, sodass davon auszugehen ist, dass sich während des Strangpressverfahrens eine Vorzugsorientierung der Kristallite eingestellt hat.

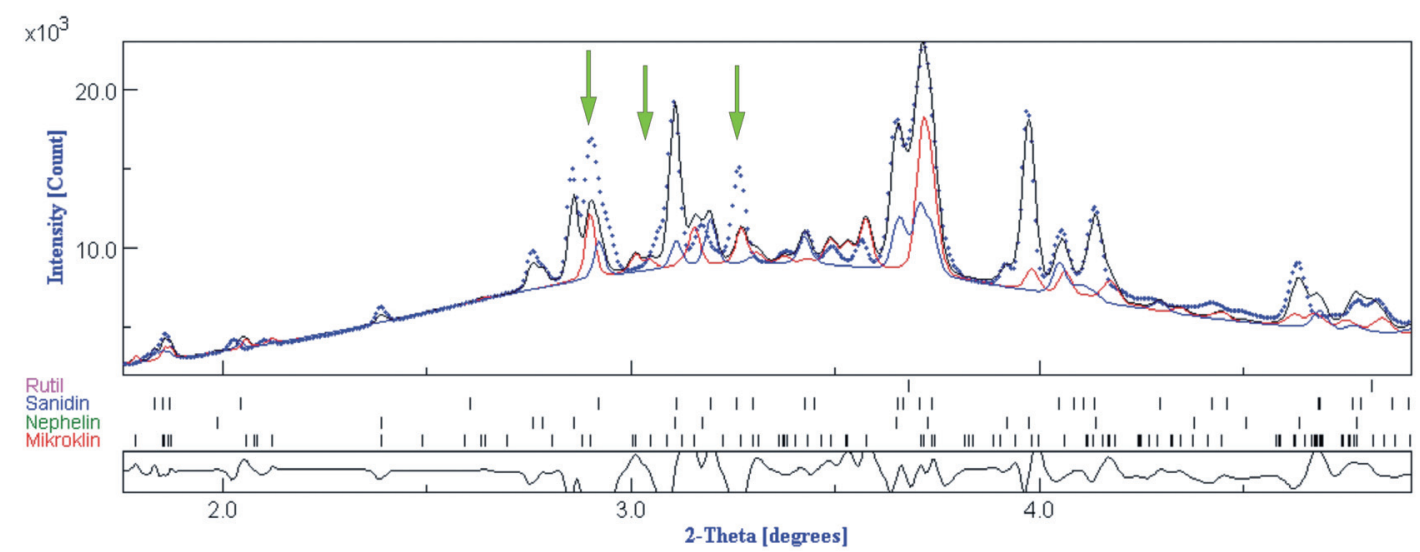

Abb. 64: Angepasstes Röntgenprofil der Dentalkeramik Vitablocs - Esthetic Line: Berechnete Intensitäten der Mikroklin- und der Sanidin-Phase unter Anwendung der E-WIMV-Methode in $\mathbf{5}^{\circ}$-Auflösung. Besonders problematisch sind die Intensitätsanpassungen der Mikroklin-Reflexe bei $2,9^{\circ}, 3,0^{\circ}$ und $3,26^{\circ}$ (Bereiche sind durch grüne Pfeile markiert). 
Die Texturart und -stärke wurde für die Silikat-Phasen in $5^{\circ}$-Auflösung nach der E-WIMVMethode mit MAUD berechnet. Dabei stellte sich heraus, dass die Kristallite der NephelinPhase mit maximal 1,3fach regellos keine bevorzugte Orientierung aufwiesen. Die Feldspatphasen Mikroklin und Sanidin hingegen zeigten Kristallvorzugsorientierungen, wobei sich die Reflexintensitäten auch unter Einbeziehung der Textur nach der E-WIMV-Methode nicht optimal anpassen ließen (Abb. 63 und 64). Zum Vergleich sind in Abb. 65 die experimentellen und die nach der E-WIMV-Methode in $5^{\circ}$-Auflösung berechneten Polfiguren der Sanidin- und der Mikroklin-Phase dargestellt, wobei Intensitätsmaxima von 1,94fach (Sanidin-Phase) und 2,01fach (Mikroklin-Phase) in den berechneten Polfiguren erreicht wurden. In den ODF-Darstellungen der Sanidin- und der Mikroklin-Phase (Abb. 66) sind in $\phi_{2}$-Richtung rotierende Orientierungsschläuche zu erkennen (Maximale Intensitäten: 3,17fach regellos für Mikroklin und 3,48fach regellos für Sanidin). 
a)
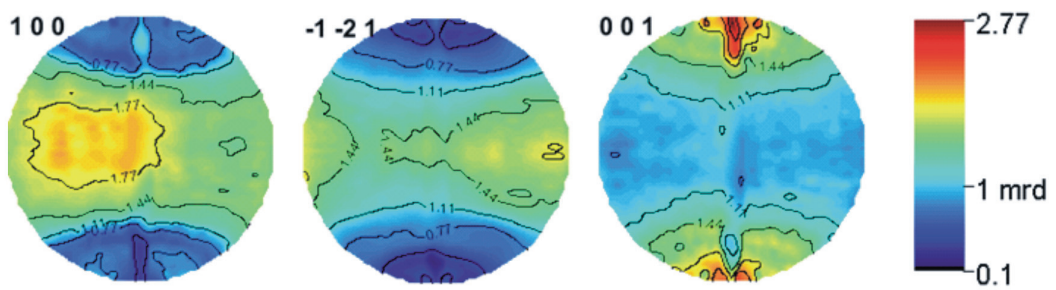

b)
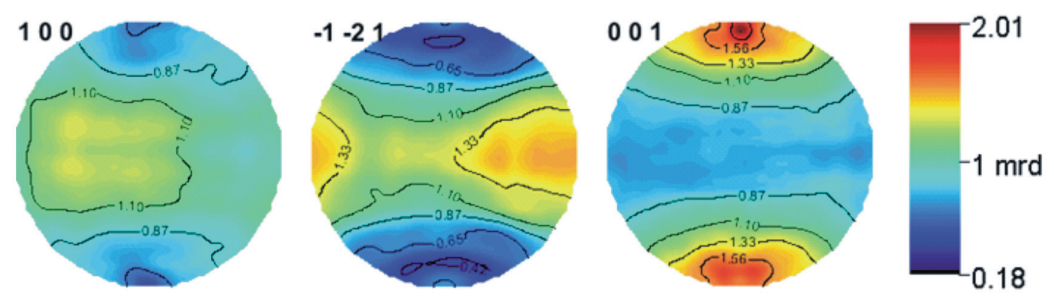

c)
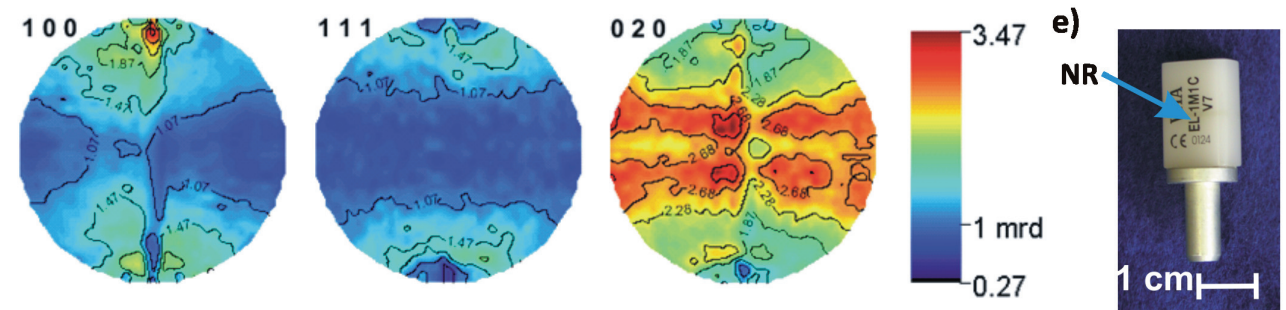

d)
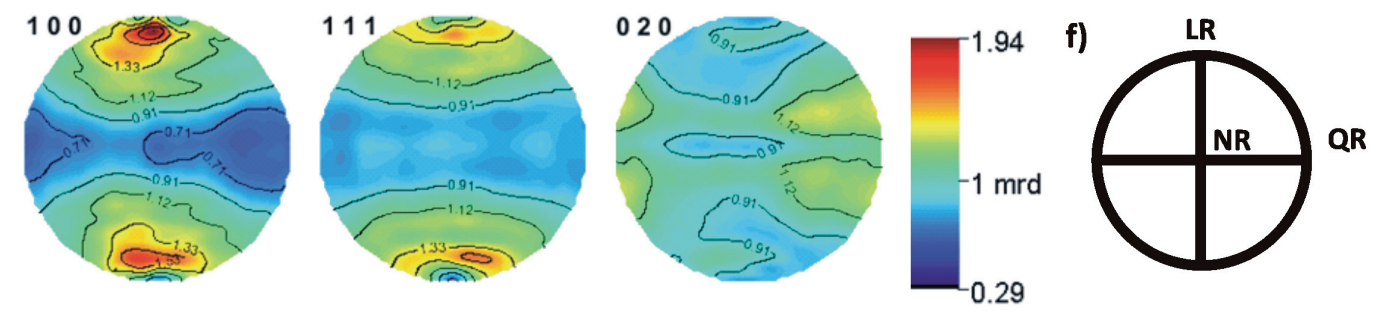

Abb. 65: Dentalkeramik Vitablocs - Esthetic Line: Experimentelle (a) und nach der EWIMV-Methode in $5^{\circ}$-Auflösung berechnete (b) Polfiguren der Mikroklin-Phase bzw. der Sanidin-Phase (c und d). In (e) ist der Messpunkt an der Keramik eingezeichnet und in (f) die Probenrichtungen (Normalrichtung NR, Querrichtung QR und Lämgsrichtung LR) innerhalb der stereographischen Projektion dargestellt. Polfiguren wurden mit trikliner (Mikroklin) bzw. monokliner (Sanidin) Kristall- und trikliner Probensymmetrie berechnet. 
a)

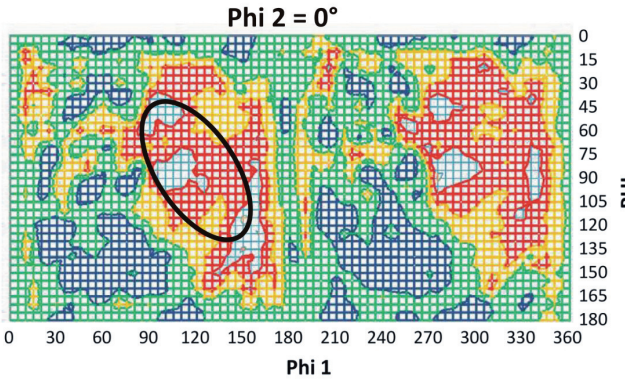

Phi $2=90^{\circ}$

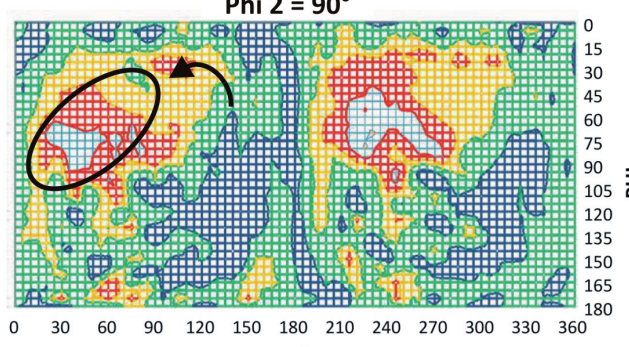

Phi 1

b)
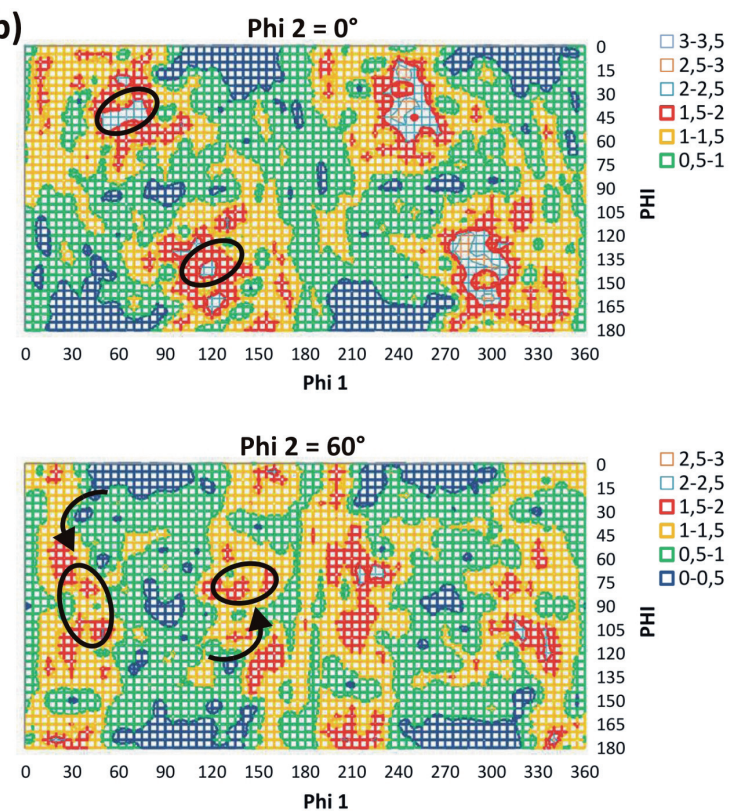

$\square 2,5-3$
$\square 2-2,5$
$\square 1,5-2$
$\square 1-1,5$
$\square 0,5-1$
$\square 0-0,5$
$\square$

$\square 2,5-3$

$\square 2,5-3$

$\square 2-2,5$

$\square 1-1,5$

망-1

폼

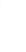

(2)
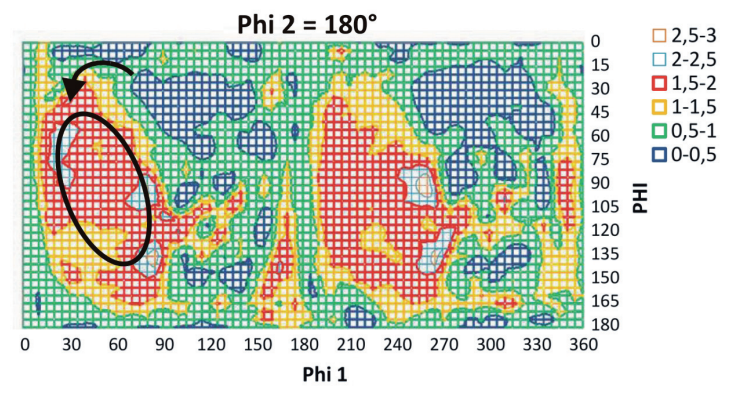

$\square 2-2,5$

$\square 1,5-2$

1-1,5 ㅁo-0,5 . 폰
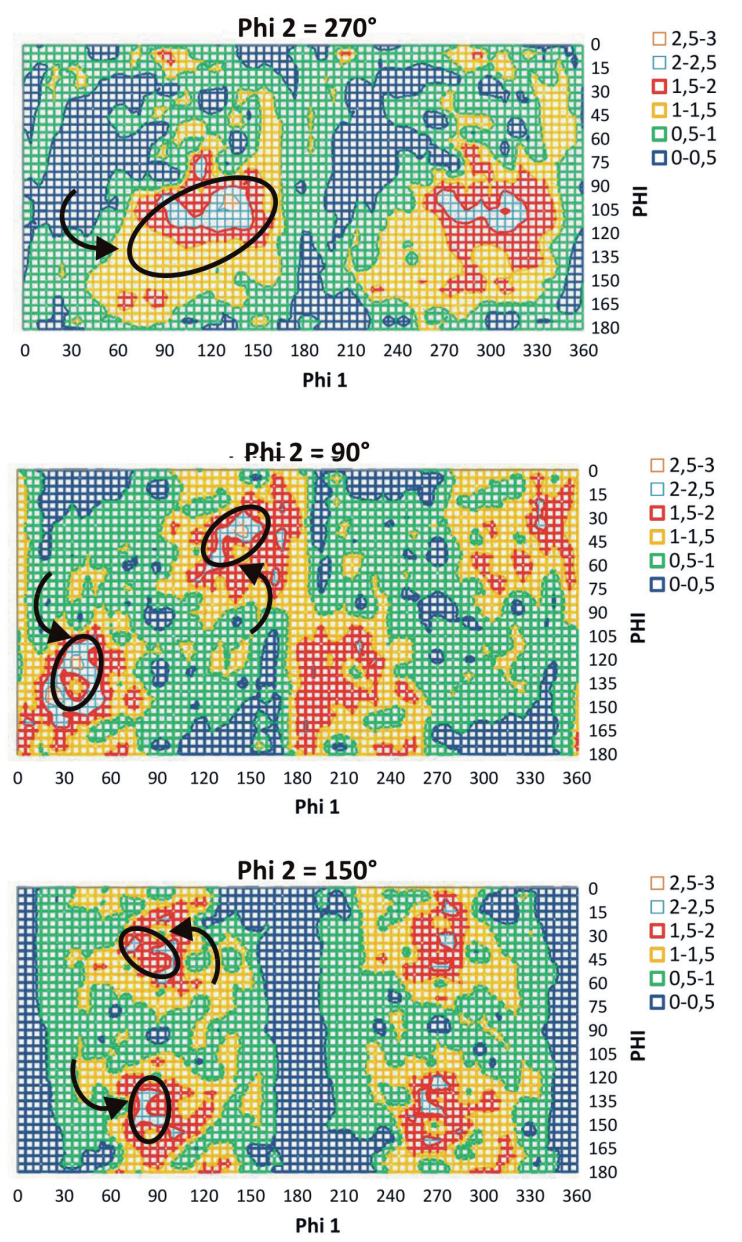

Abb. 66: Ausgewählte $\phi_{2}$-Schnitte für Phasen der Dentalkeramik Vitablocs - Esthetic Line: (a) Mikroklin-Phase (trikline Kristallsymmetrie; $\phi_{2}=0^{\circ}, 90^{\circ}, 180^{\circ}$ und $270^{\circ}$ ), (b) SanidinPhase (monokline Kristallsymmetrie; $\phi_{2}=0^{\circ}, 60^{\circ}, 90^{\circ}$ und $150^{\circ}$ ). Bei beiden Phasen sind Orientierungsschläuche zu sehen, die sich schraubenartig durch die gesamte ODF ziehen. Um den Verlauf der Orientierungsschläuche in der ODF zu verdeutlichen, sind die Intensitätsmaxima des jeweiligen Orientierungsschlauchs schwarz eingekreist sowie deren Verlagerung durch schwarze Pfeile markiert. Außerdem fällt auf, dass sich die Intensitäten nach $180^{\circ}$ in $\phi_{1}$ nahezu wiederholen. Dieses deutet auf eine fast monokline Probensymmetrie hin. 


\subsubsection{Glasinfiltrationskeramik Vita In-Ceram Alumina}

An einer ca. $2 \mathrm{~mm}$ dicken Scheibe dieser Korund-Keramik wurden im vorgesinterten und im La-glasinfiltrierten Zustand konventionelle Polfiguren gemessen und sowohl nach Bunge (1993) als auch mit der E-WIMV-Methode ausgewertet. In beiden Fällen wurde eine regellose Textur festgestellt (maximal 1,2fach regellos; Beispiel für 2d-Transmissionsbild s. Anhang Abb. B.17).

\subsubsection{Glasinfiltrationskeramik Vita In-Ceram Zirkonia}

Diese mit tetragonalem $\mathrm{Ce}-\mathrm{ZrO}_{2}$-verstärkte Korund-Keramik zeigte sowohl vor als auch nach der Glasinfiltration bei $\approx 1100^{\circ} \mathrm{C}$ bei Anwendung der Textur-Analyse nach der E-WIMV-Methode in 3,75-Auflösung eine leichte Vorzugsorientierung der Korund-Kristallite (Polfiguren in Abb. 67), während die Kristallite der $\mathrm{Ce}-\mathrm{ZrO}_{2}$-Phase regellos verteilt waren. Der Glasinfiltrationsvorgang bewirkte keine Änderung in der Vorzugsorientierung der Korund-Kristallite. In beiden Zuständen war eine leichte (001)-Fasertextur in der ODF erkennbar (Abb. 68).

a)
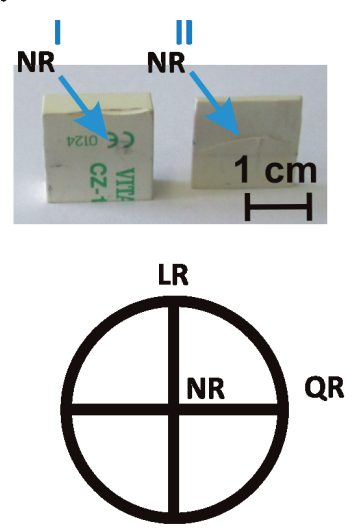

b)

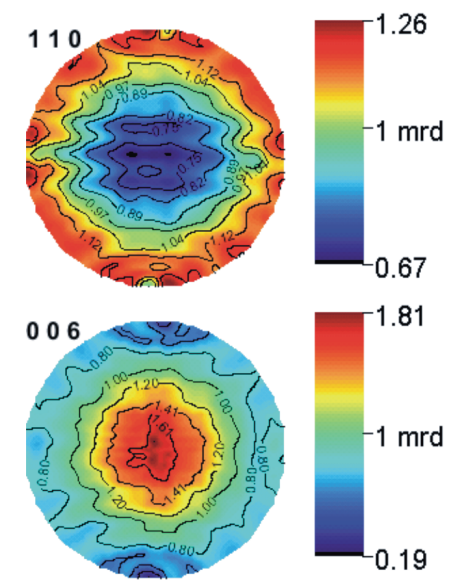

c)

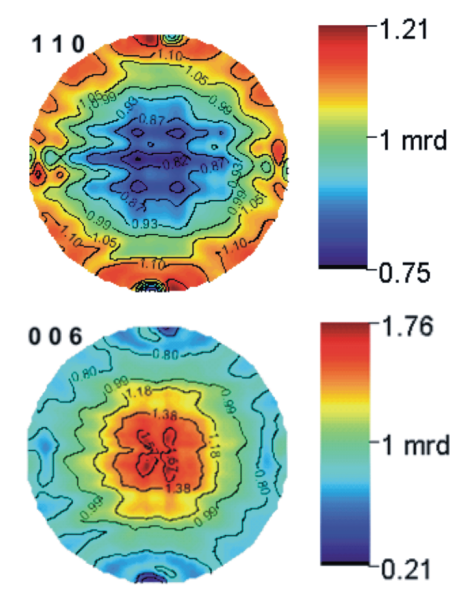

Abb. 67: Gerüstkeramik Vita In-Ceram Zirkonia.
(a) Darstellung der Messpunkte (oben) und der Richtungen (Normalrichtung NR, Längsrich- tung LR und Querrichtung QR) innerhalb der stereographischen Projektionen (unten).
(b) Berechnete Polfiguren der Korund-Phase vor der Infiltration mit La-Glas (Messpunkt I; E-WIMV-Methode in $3,75^{\circ}$-Auflösung)
(c) Berechnete Polfiguren der Korund-Phase nach der Glasinfiltration (Messpunkt II; E- WIMV-Methode in $3,75^{\circ}$-Auflösung).


a)

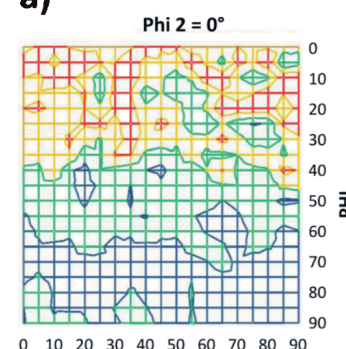

Phi 1

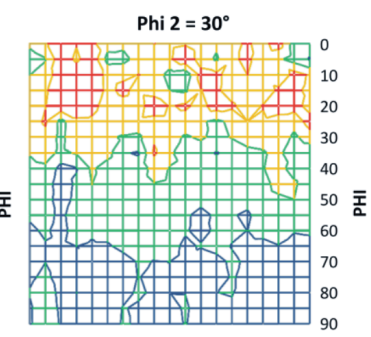

Phi 1 b)

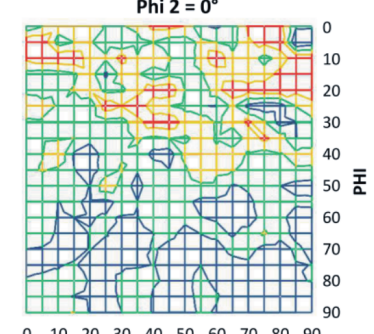

Phi 1

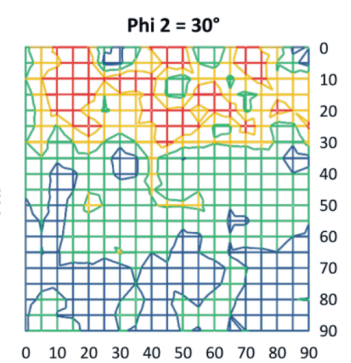

Phi 1

Abb. 68: Ausgewählte $\phi_{2}$-Schnitte für die Korund-Phase der Dentalkeramik Vita In-Ceram Zirkonia: (a) im vorgesinterten Zustand $\left(\phi_{2}=0^{\circ}, 30^{\circ}\right)$ und (b) im La-glasinfiltrierten Zustand $\left(\phi_{2}=0^{\circ}, 30^{\circ}\right)$. Die maximalen Intensitäten liegen bei 4,6 fach regellos im vorgesinterten Zustand und 4,0fach regellos im fertigen Zustand. Berechnet mit hexagonaler Kristall- und trikliner Probensymmetrie.

\subsubsection{Goldlegierungen Degudent $U$ und Degulor $M$}

Von der aufbrennfähigen Legierung Degudent $U$ wurden an einer Gussscheibe mit Keramikverblendung Texturmessungen am Synchrotronmessplatz BW5 vorgenommen. Allerdings erwies sich die Absorption der Goldlegierung bei der verwendeten Strahlungsenergie von $\approx 100 \mathrm{keV}$ als zu hoch: Bei zunehmendem Drehwinkel $\omega$ und somit zunehmender Weglänge in der Goldlegierung wurde die Synchrotronstrahlung so stark absorbiert, dass nur ein sehr kleiner Winkelbereich $-36^{\circ}$ $\leq \omega \leq+36^{\circ}$ für die Texturanalyse verwendet werden konnte. Dennoch wurde eine Texturrechnung dieser Probe in $2,5^{\circ}$ - und in $7,5^{\circ}$-Auflösung vorgenommen. Das Ergebnis der Texturanalyse war, dass die Hauptphase $\alpha$-AuPdPt regellos orientiert ist, wobei zu beachten ist, dass die AuPd-Pt-Phase sehr grobkörnig war und somit eine geringe Kornstatistik aufwies (Poliguren s. Abb. $69)$.

Die Scheiben aus den Gusskanälen beider Goldlegierungen zeigten eine noch größere Grobkörnigkeit, sodass weder für Degudent $U$ noch für Degulor $M$ eine Texturanalyse an diesen Proben durchgeführt werden konnte.
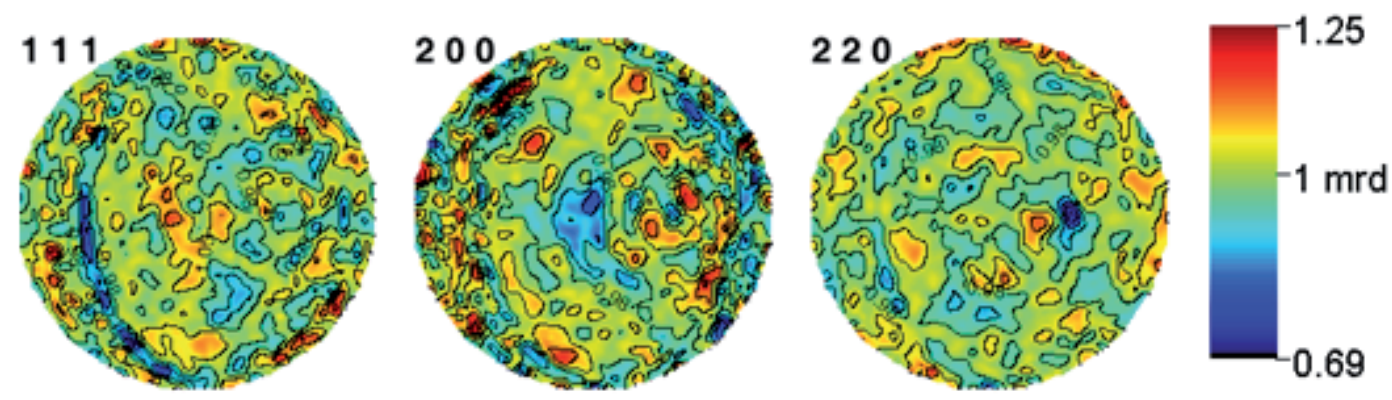

Abb. 69: Nach der E-WIMV-Methode in 2,5 ${ }^{\circ}$-Auflösung zurückberechnete Polfiguren von der Au-Pd-Pt-Phase der Goldlegierung Degudent $U$. 


\subsubsection{Titan-Wurzelstift}

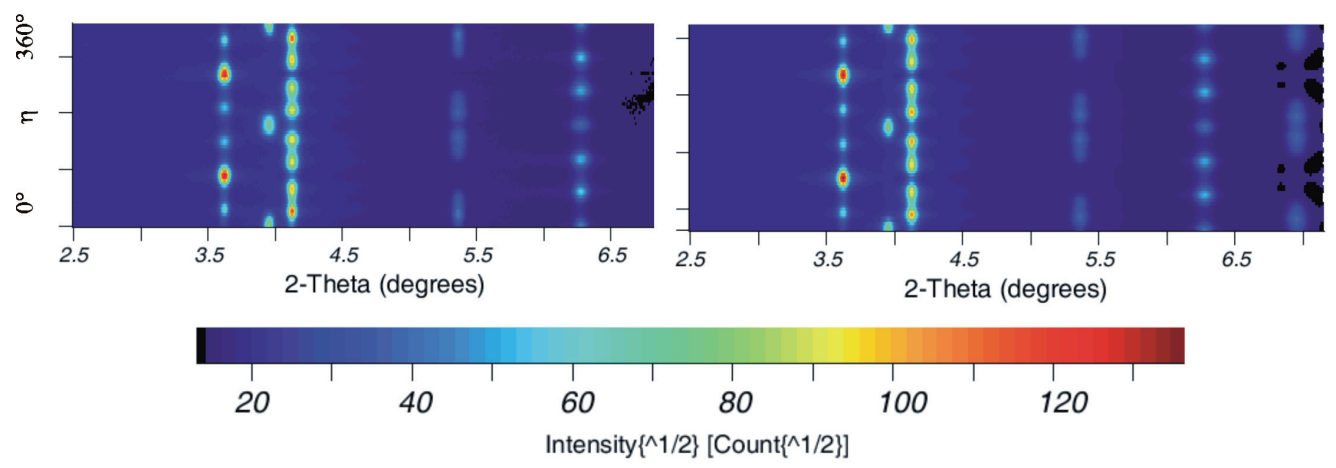

Abb. 70: Beispiel für die kombinierte Textur-Rietveld-Analyse des Titan-Wurzelstiftes der Firma Gebr. Brasseler (Messung an der Probenmitte). Links: Original-Datensatz; rechts: angepasstes Röntgenprofil.

Der Titan-Wurzelstift wurde in der Mitte und am Gewinde mittels Synchrotron- und konventioneller Strahlung auf Textur untersucht. Die Auswertung der Synchrotrondaten (2dTransmissionsbilder; BW5) erfolgte im ersten Schritt mit MAUD unter Anwendung der E-WIMVMethode ( $5^{\circ}$-Auflösung der ODF, ohne Annahme einer Probensymmetrie; Beispiel der Profilanpassung in Abb. 70). An beiden Stellen ließs sich eine ausgeprägte $\Phi$-Faser bei $\phi_{1}=\phi_{2}=0^{\circ}$ $(g=\{0,0-90,0\}=(h k i l)\langle 10 \overline{1} 0\rangle)$ beobachten. Dabei wurden maximale Intensitäten von 11fach regellos in den zurück berechneten Polfiguren und von 18fach regellos in der ODF erreicht (Abb. 71 und 72). Neben der $(h k i l)\langle 10 \overline{1} 0\rangle$-Faser war eine weitere Texturkomponente in der Nähe von $g=\{60,0,0\}=(0001)\langle 1 \overline{1} 00\rangle$ vorhanden. Insbesondere die Fasertextur deutet daraufhin, dass der Titanwurzelstift durch Drahtziehen hergestellt wurde. Außerdem zeigt die Formgebung am unteren Ende des Stiftes, dass der Stift nach dem Drahtziehen durch Schmieden in seine endgültige Form gebracht wurde.

Zusätzlich wurde die Textur anhand von konventionellen Polfigurmessungen (100, 002, 101, 102, $\left.110 ; l_{\max }=12\right)$ am PTS Seifert nach Bunge (1993) unter Annahme einer orthorhombischen Probensymmetrie berechnet, wobei der Strahl parallel zur Längsachse des Ti-Stiftes ausgerichtet war (Messungen am Synchrotron: senkrechte Ausrichtung zur Längsachse). Qualitativ stimmen die Ergebnisse der konventionellen Texturmessungen zum Teil mit den Synchrotronmessungen überein, wobei berücksichtigt werden muss, dass der Betrachtungswinkel um $90^{\circ}$ gedreht ist. Die Texturkomponente $g=\{0-90,0,0\}=(0001)\langle u v t w\rangle$ entspricht dabei der Texturkomponente $g=\{0,0-90,0\}=(h k i l)\langle 10 \overline{1} 0\rangle$ aus Synchrotrondaten (vgl. Abb. 72 und 74 ). Neu ist die Texturkomponente $g=\{0-90,90,30\}=(01 \overline{1} 0)\langle u v t w\rangle$. Unterschiede treten auch in der Texturstärke auf: Während die aus Synchrotrondaten nach der E-WIMV-Methode berechneten Polfiguren (Abb. 71) Werte bis zu 11fach regellos annahmen (in ODF: 18fach), wurden nach der harmonischen Methode - angewendet auf die konventionelle Polfigurmessungen - nur Werte von 4,1fach regellos in den Polfiguren (Abb. 73) und von 4,6fach in der ODF (Abb. 74) erreicht. 
a)
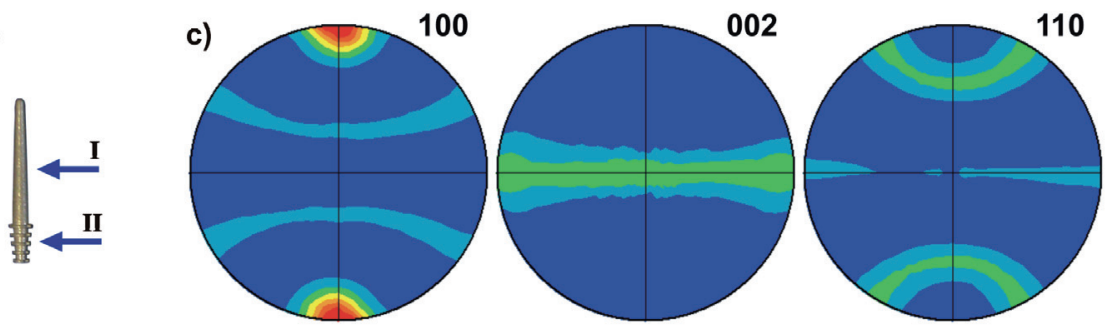

b)
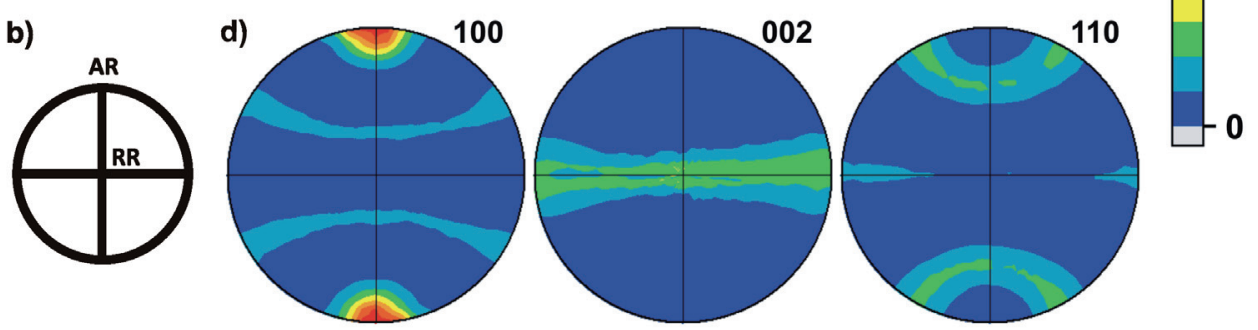

Abb. 71: Nach der E-WIMV-Methode berechnete Polfiguren des Titan-Wurzelstifts der Firma Gebr. Brasseler (Synchrotrondaten, $5^{\circ}$-Auflösung der ODF; hexagonale Kristall- und trikline Probensymmetrie).
(a) Darstellung der Messpunkte von (c) und (d).
(b) Lage der Axial- und Radialrichtung (AR bzw. RR) in der stereographischen Projektion.
(c) Polfiguren vom Messpunkt I (BW5; $\lambda=0,16134 \AA)$.
(d) Polfiguren vom Messpunkt II (BW5; $\lambda=0,12398 \AA)$.

Des Weiteren wurden 6 experimentelle Polfiguren aus MAUD (002, 101, 102, 103, 110, 200) zur Texturberechnung nach Bunge (1993) verwendet. Die zurück berechneten Polfiguren erreichten Werte um 11fach regellos (Abb. 75) und die ODF um 12fach regellos (Abb. 76). Die Texturkomponenten $g=\{0,0-90,0\}=(h k i l)\langle 10 \overline{1} 0\rangle$ und in der Nähe von $g=\{60,0,0\}=(0001)\langle 1 \overline{1} 00\rangle$ stimmen gut mit den Ergebnissen nach der E-WIMV-Methode überein. 

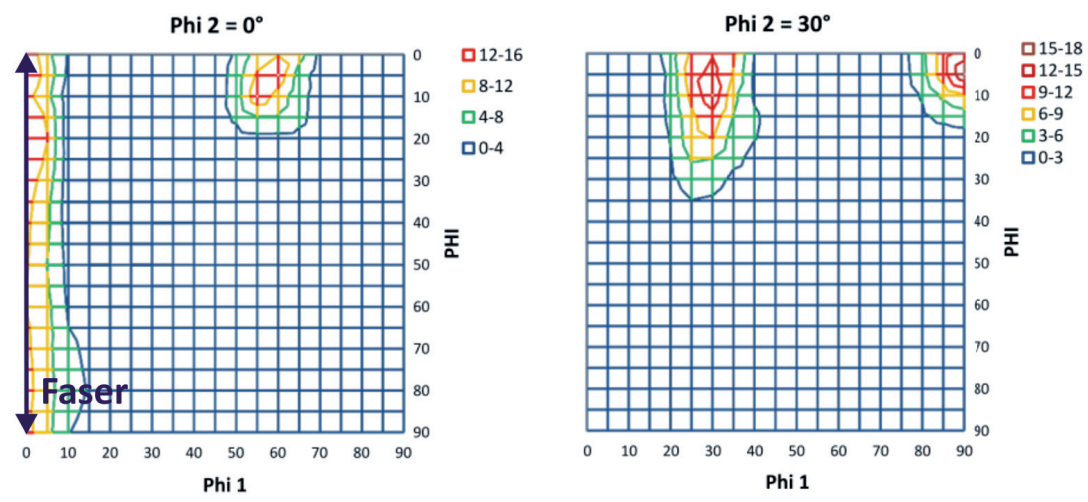

Abb. 72: Ausgewählte $\phi_{2}$-Schnitte aus der nach der E-WIMV-Methode berechneten ODF für den Titan-Wurzelstift der Firma Gebr. Brasseler (Synchrotrondaten; Messung an Probenmitte/Messpunkt I; $\left.\phi_{2}=\mathbf{0}^{\circ}, \mathbf{3 0}^{\circ}\right)$. Texturkomponenten: $g=\{0,0-90,0\}=$ (hkil) $\langle 10 \overline{1} 0\rangle$ und in der Nähe von $g=\{60,0,0\}=(0001)\langle 1 \overline{1} 00\rangle$. Berechnet mit hexagonaler Kristall- und trikliner Probensymmetrie.

a)

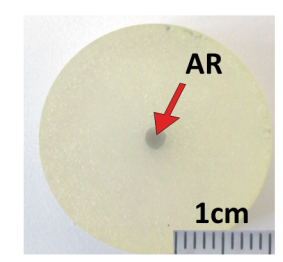

b)

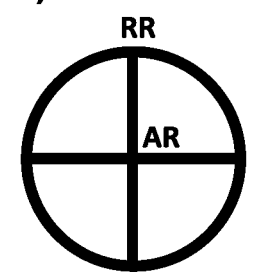

c)

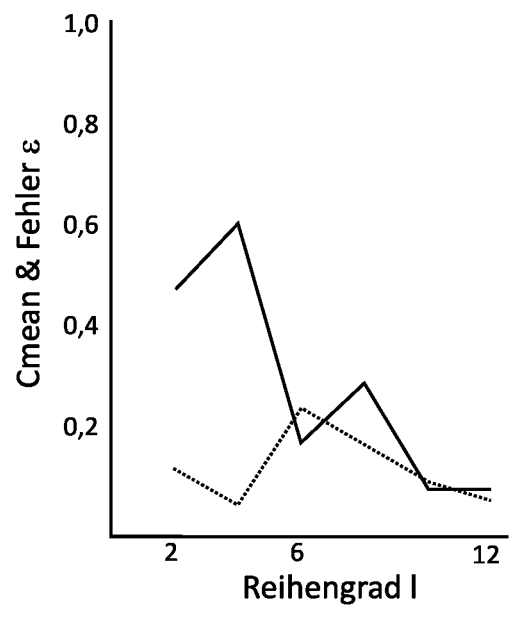

d)
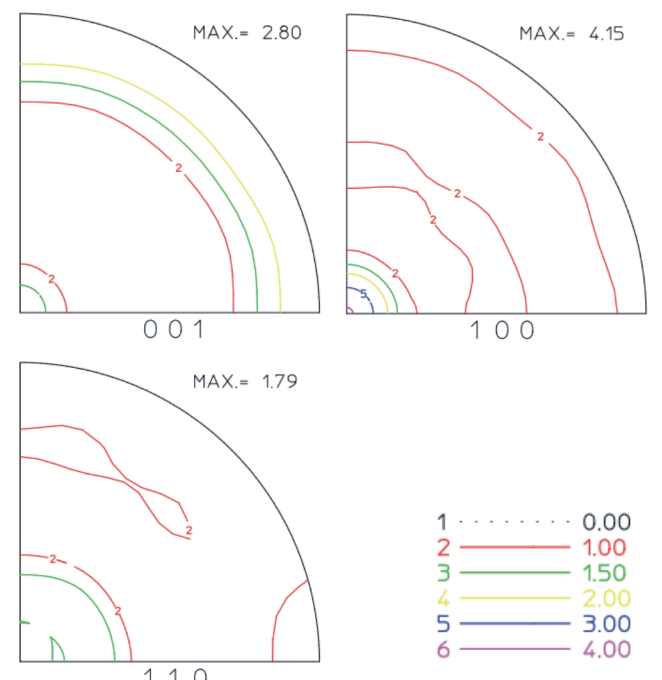

Abb. 73: Nach der harmonischen Methode berechnete Polfiguren des Titan-Wurzelstifts der Firma Gebr. Brasseler ( $l=12$; Daten aus konventioneller Polfigurmessung

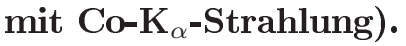

(a) Eingebettete Probe mit eingezeichneter Strahlrichtung.

(b) Lage der Axial- und Radial-Richtung (AR bzw. RR) in der stereographischen Projektion.

(c) Verlauf der mittleren, absoluten C-Werte $C_{\text {mean }}$ und des Fehlers $\epsilon$.

(d) Berechnete Polfiguren ( $\frac{1}{4}$-Ausschnitt für orthorhombisch berechnete Probensymmetrie ausreichend). 

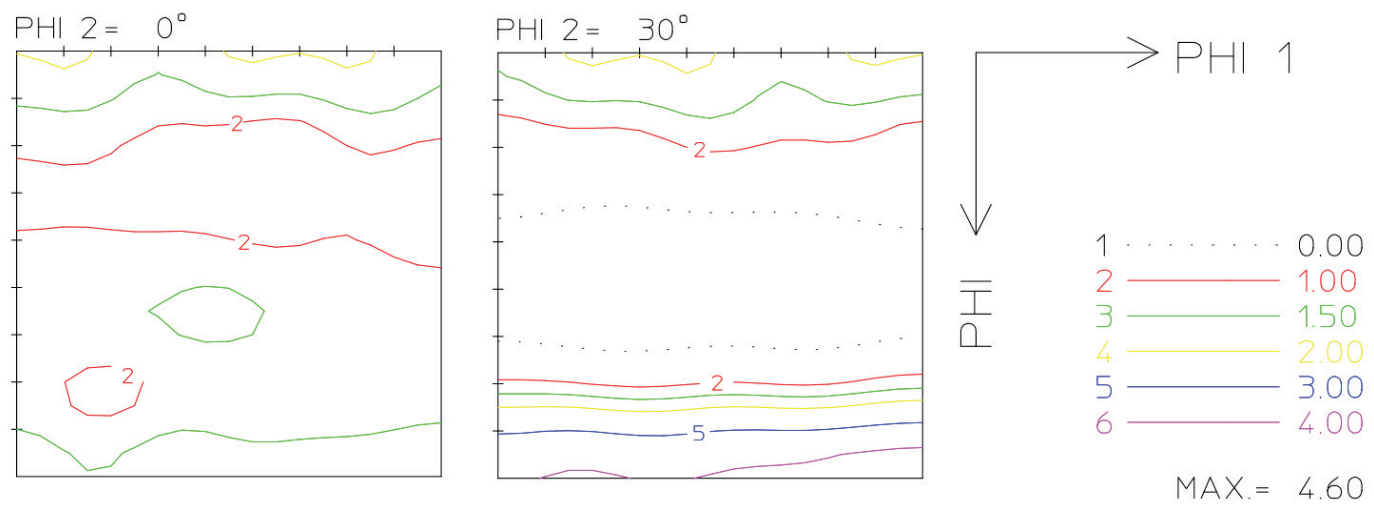

Abb. 74: Nach der Methode der symmetrischen verallgemeinerten Kugelfunktionen berechnete Orientierungsverteilung des Titan-Wurzelstifts der Firma Gebr. Brasseler ( $l=12$; Daten aus konventioneller Polfigurmessung mit $\mathbf{C o -} \mathbf{K}_{\alpha}$-Strahlung). Texturkomponenten: $g=\{0-90,0,0\}=(0001)\langle u v t w\rangle$ und $g=\{0-90,90,30\}=(01 \overline{1} 0)\langle u v t w\rangle$. Berechnet mit hexagonaler Kristall- und orthorhombischer Probensymmetrie.

a)

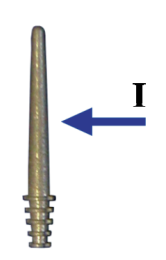

b)

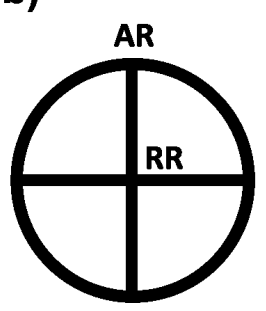

c)
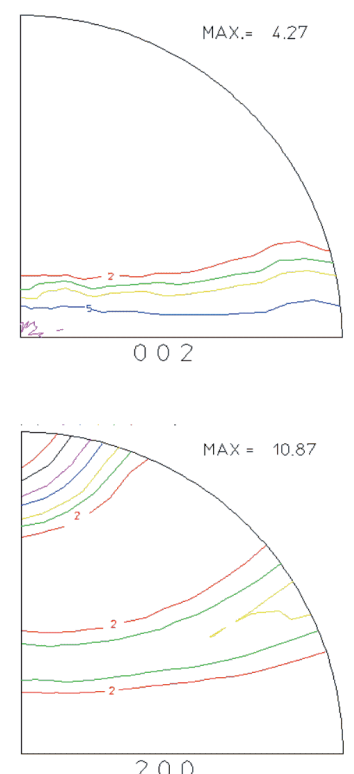

d)
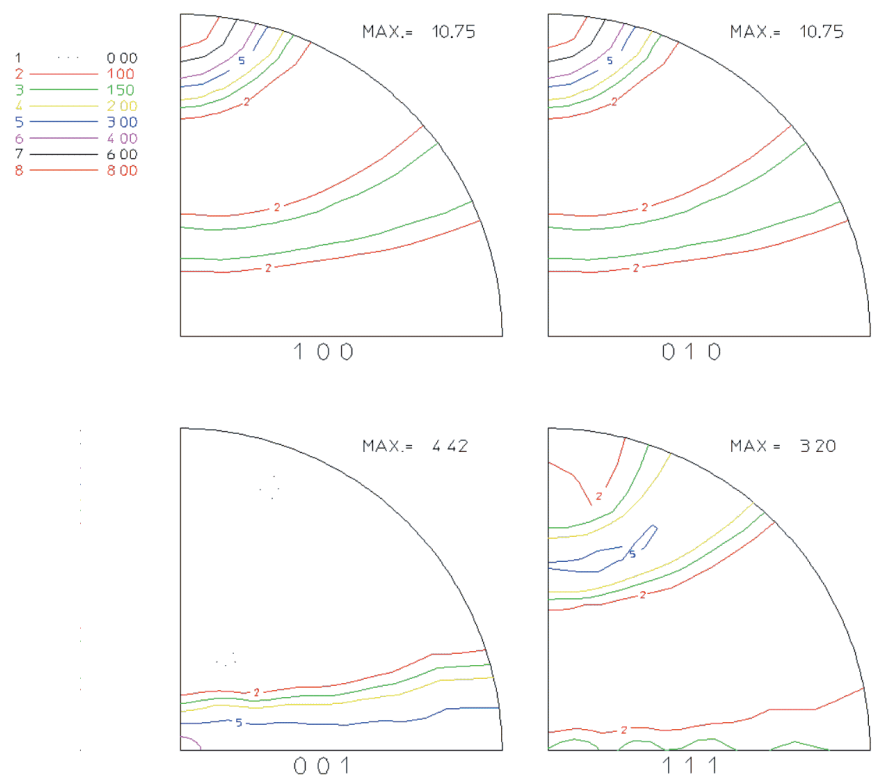

Abb. 75: Nach der Methode der symmetrischen verallgemeinerten Kugelfunktionen berechnete Polfiguren des Titan-Wurzelstifts der Firma Gebr. Brasseler (Synchrotrondaten).

(a) Probe mit eingezeichneter Strahlrichtung (gleiche Messung wie in Abb. 71).

(b) Lage der Axial- und Radial-Richtung (AR bzw. RR) in der stereographischen Projektion.

(c) Experimentelle Polfiguren nach MAUD.

(d) Nach der konventionellen Methode berechnete Polfiguren ( $\frac{1}{4}$-Ausschnitt für orthorhombisch berechnete Probensymmetrie ausreichend). 
a)

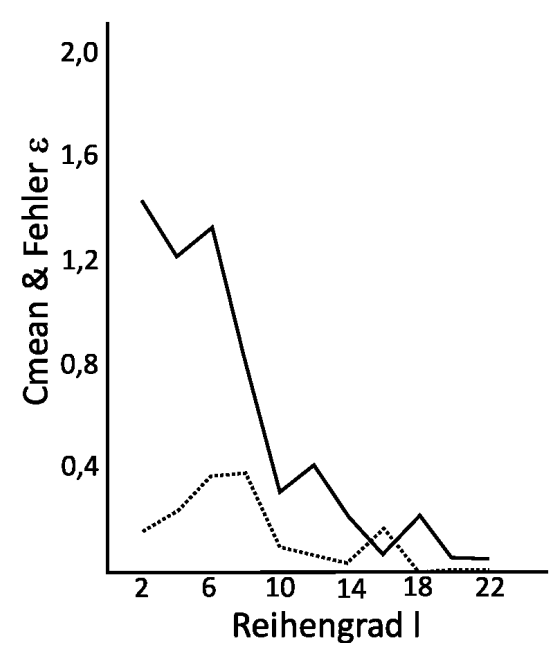

b)
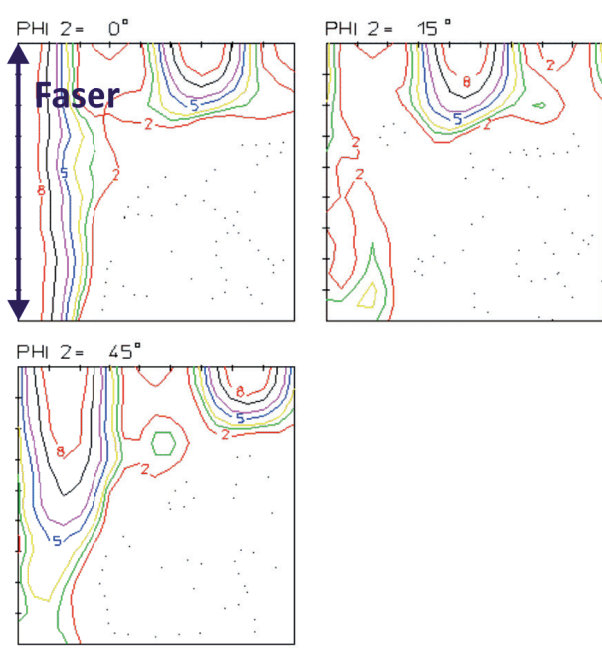
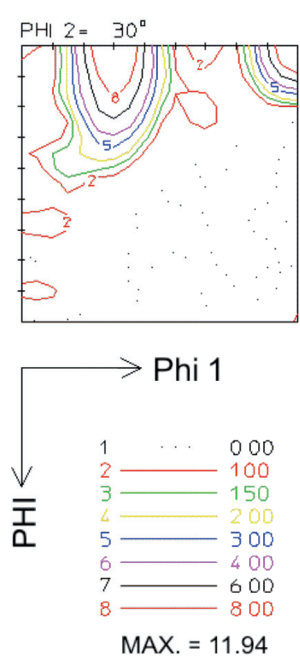

Abb. 76: Nach der Methode der symmetrischen verallgemeinerten Kugelfunktionen berechnete Orientierungsverteilung des Titan-Wurzelstifts der Firma Gebr. Brasseler (Synchrotrondaten). Texturkomponenten: $g=\{0,0-90,0\}=(h k i l)\langle 10 \overline{1} 0\rangle$ und in der Nähe von $g=\{60,0,0\}=(0001)\langle 1 \overline{1} 00\rangle$. Berechnet mit hexagonaler Kristall- und orthorhombischer Probensymmetrie.

(a) Verlauf der mittleren, absoluten C-Werte $C_{\text {mean }}$ und des Fehlers $\epsilon$.

(b) Orientierungsverteilung im Eulerraum. 


\subsubsection{Titan-Implantatsystem OsseoSpeed}

Das Titan-Implantat Osseospeed wurde an zwei Stellen mittels Synchrotron-Transmissionsaufnahmen (BW5; $\lambda=0,12137 \AA$ ) mit der in MAUD implementierten E-WIMV-Methode auf Textur hin untersucht (Polfiguren in Abb. 77), wobei die Messung am Messpunkt II an massivem Titan durchgeführt wurde, während am Messpunkt I sehr wahrscheinlich der Hohlraum des Ti-Innenschraubgewindes mitgemessen wurde (Abb. 77a). Exemplarisch sind in Abb. 78 die experimentellen und berechneten Polfiguren für den Messpunkt II dargestellt, die eine gute Übereinstimmung zeigen. Minimale Unterschiede sind auf die leicht ausgeprägte Grobkörnigkeit der Titankristallite zurückzuführen.

a)

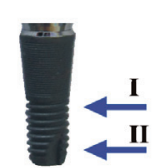

b)

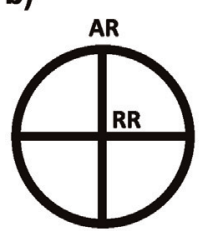

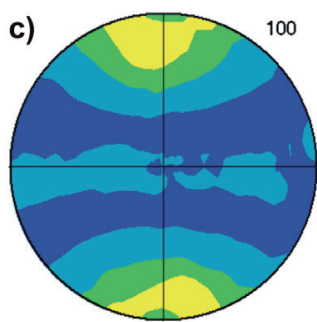

d)

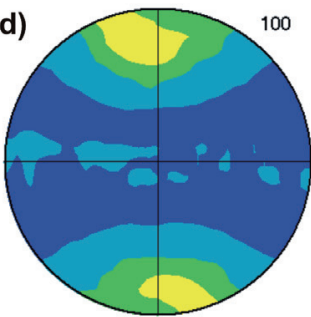

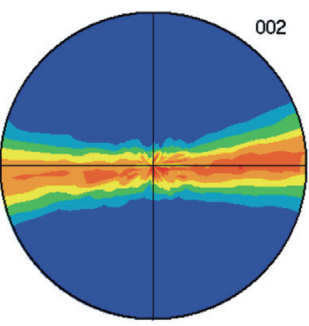
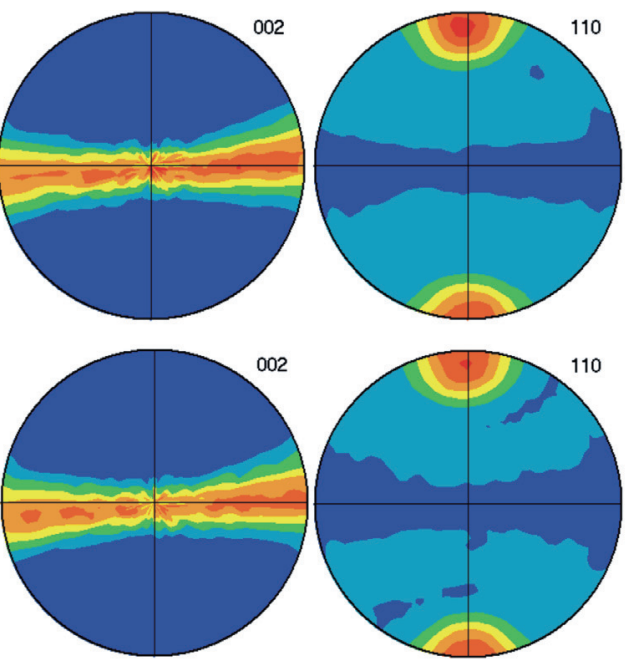

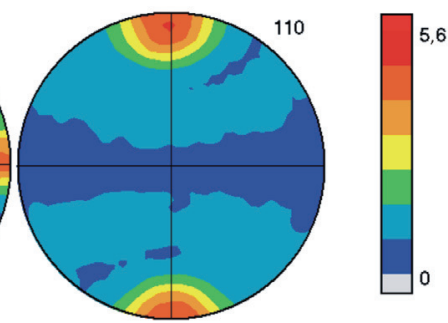

Abb. 77: Nach der WIMV-Methode in $5^{\circ}$-Auflösung berechnete Polfiguren des TitanImplantates Osseospeed der Firma Astra (berechnet mit hexagonaler Kristallund trikliner Probensymmetrie).

(a) Darstellung der Messpunkte I und II.

(b) Lage der Axial- und Radialrichtung (AR bzw. RR) in der stereographischen Projektion.

(c) Polfiguren vom Messpunkt I.

(d) Polfiguren vom Messpunkt II.

Anhand der berechneten Polfiguren (Abb. 77) sowie an den ODFs ist zu erkennen, dass die Textur an beiden Messpunkten ähnlich war. Stellvertretend für beide Messpunkte ist die ODF des Messpunkts II in Abb. 79 dargestellt, in der eine $\Phi$-Faser bei $\phi_{1}=0^{\circ}$ und $\phi_{2}=30^{\circ}$ $(g=\{0,0-90,30\}=(h k i l)\langle 2 \overline{1} \overline{1} 0\rangle)$ zu sehen ist. Ebenso ist hier eine weitere Texturkomponente bei $g=\{60,0,0\}=(0001)\langle 1 \overline{1} 00\rangle$ sichtbar. Wie schon beim Titan-Wurzelstift deutet das Vorkommen einer Fasertextur sowie die endgültige Formgebung daraufhin, dass das Implantat durch Drahtziehen und anschließendes Schmieden hergestellt wurde. 
a)

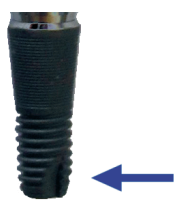

b)

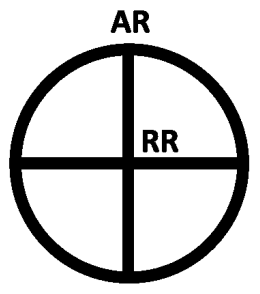

c)

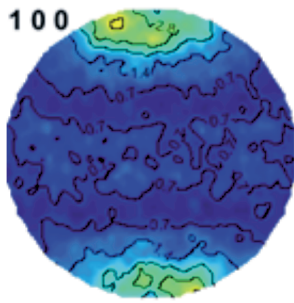

d)

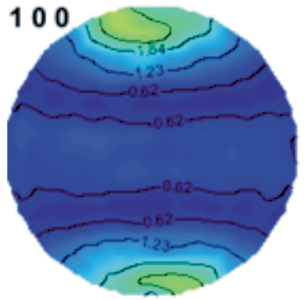

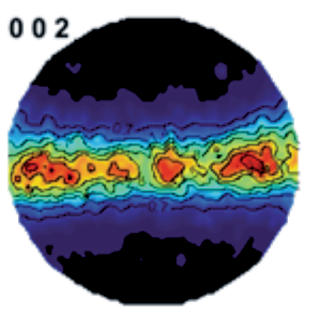
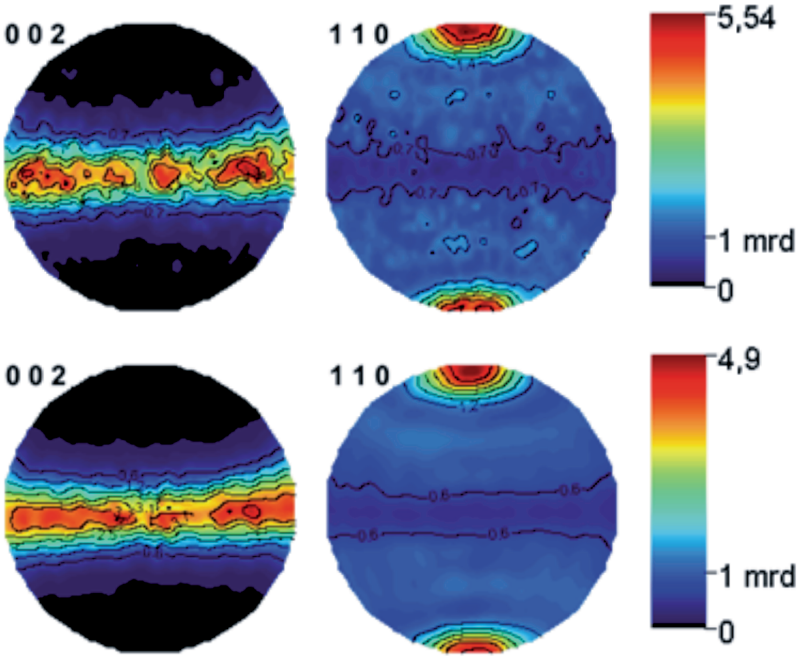

Abb. 78: Experimentelle und nach der E-WIMV-Methode in 3,75 ${ }^{\circ}$-Auflösung berechnete Polfiguren des Titan-Implantates Osseospeed der Firma Astra (berechnet mit hexagonaler Kristall- und trikliner Probensymmetrie).

(a) Darstellung des Messpunkts II.

(b) Lage der Axial- und Radialrichtung (AR bzw. RR) in der stereographischen Projektion.

(c) Experimentelle Polfiguren.

(d) Berechnete Polfiguren (E-WIMV-Methode).
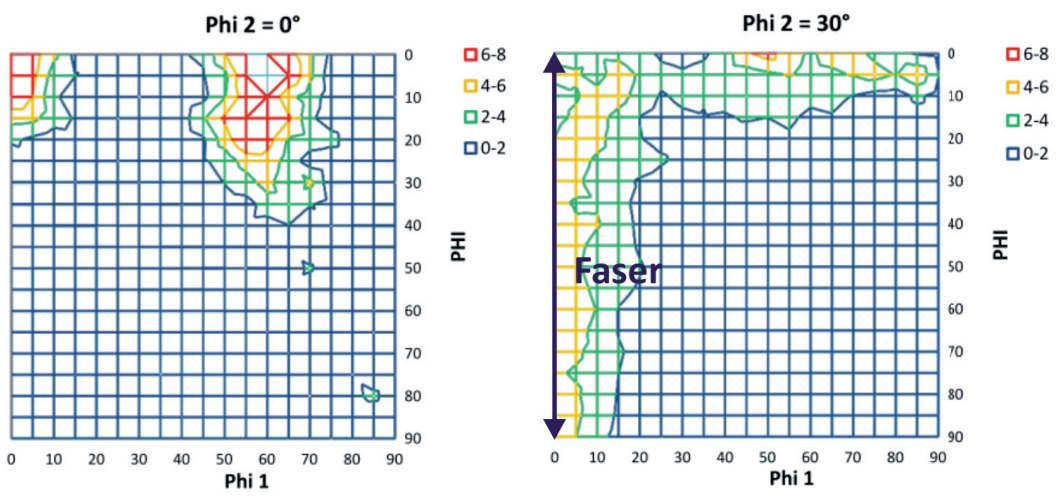

Abb. 79: Ausgewählte $\phi_{2}$-Schnitte aus der nach der E-WIMV-Methode berechneten ODF für das Titan-Implantat Osseospeed (Messpunkt II; $\phi_{2}=\mathbf{0}^{\circ}, \mathbf{3 0}^{\circ}$ und $\mathbf{6 0}{ }^{\circ}$ ). Texturkomponenten: $g=\{0,0-90,30\}=($ hkil $)\langle 2 \overline{1} \overline{1} 0\rangle$ und in der Nähe von $g=\{60,0,0\}=$ (0001) $\langle 1 \overline{1} 00\rangle$. Berechnet mit hexagonaler Kristall- und trikliner Probensymmetrie. 


\subsection{Berechnung der anisotropen physikalischen Eigenschaften aus Texturanalysen}

Im Folgenden soll ein Überblick über die Auswirkung der Kristallitorientierungen einzelner Mineralphasen auf die makroskopischen physikalischen Eigenschaften der Zahnersatzwerkstoffe gegeben werden.

\subsubsection{Amalgame Amalcap regular und fast}

Da in der Texturanalyse keine Kristallitvorzugsorientierungen festgestellt werden konnten, sind die makroskopischen physikalischen Eigenschaften dieser Amalgame als isotrop anzunehmen. Die thermische Ausdehnung der Amalcap-Amalgame sollten insgesamt bei $25,5 \cdot 10^{-6} / \mathrm{K}$ liegen (Kandil et al., 1989). Für die elastischen Eigenschaften sind isotrope Werte der einzelnen Phasen in der Literatur zu finden (Grenoble \& Katz, 1971; Ghosh, 2004). Demnach hat die Hauptphase $\mathrm{Ag}_{2} \mathrm{Hg}_{3}$ einen isotropen E-Modul von $74 \mathrm{GPa}$, die $\mathrm{Ag}_{3}$ Sn-Phase einen von 81,4 GPa und die $\mathrm{Cu}_{6} \mathrm{Sn}_{5}$-Phase einen von 96,9 $\mathrm{GPa}$.

\subsubsection{Dentalkomposite, Prothesenkunststoffe und Glasionomerzement}

Die physikalischen Eigenschaften dieser Gruppe wurden nicht aus den Ergebnissen der Texturanalyse berechnet, da entweder keine kristallinen Anteile vorhanden waren oder deren Gewichtsanteil nur sehr gering war. Die Zinksulfatzemente bestanden zwar nur aus kristallinen Komponenten, waren aber in ihrer chemisch-mineralogischen Zusammensetzung so komplex, dass keine Texturrechnungen vorgenommen und somit auch keine Anisotropie in den makroskopischen physikalischen Eigenschaften berechnet werden konnten.

\subsubsection{Y- $\mathrm{ZrO}_{2}$-Keramik Cercon base}

Da sich diese Keramik zu 100 Gew.\% aus der regellos angeordneten $\mathrm{Y}_{-} \mathrm{ZrO}_{2}$-t-Phase zusammensetzte, sind ihre physikalischen Eigenschaften - wie von der Firma angenommen - als isotrop anzunehmen (Thermische Ausdehnung: 10,5·10-6/K; E-Modul: 210 GPa; DeguDent, 2013).

\subsection{4 $\mathrm{Li}_{2} \mathrm{Si}_{2} \mathrm{O}_{5}$-Keramik IPS e.max Press}

Für diese LS2-Keramik konnte nur die anisotrope thermische Ausdehnung der Hauptphase $\mathrm{Li}_{2} \mathrm{Si}_{2} \mathrm{O}_{5}$ berechnet werden, da insgesamt nur sehr wenige Studien zu den physikalischen Eigenschaften dieser Silikatverbindungen vorlagen. Für die $\mathrm{Li}_{3} \mathrm{PO}_{4}$-Phase, die mit ca. $10 \mathrm{Gew} . \% \mathrm{im}$ Endzustand den zweitgrößten Gewichtsanteil ausmachte, waren keine Einkristalldaten über die thermische Ausdehnung oder über den E-Modul vorhanden. 
a)

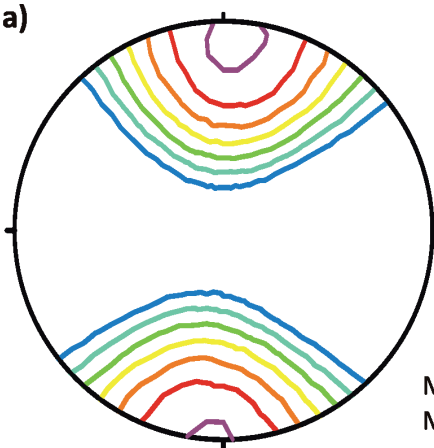

c)

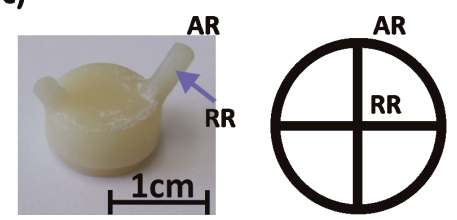

b)

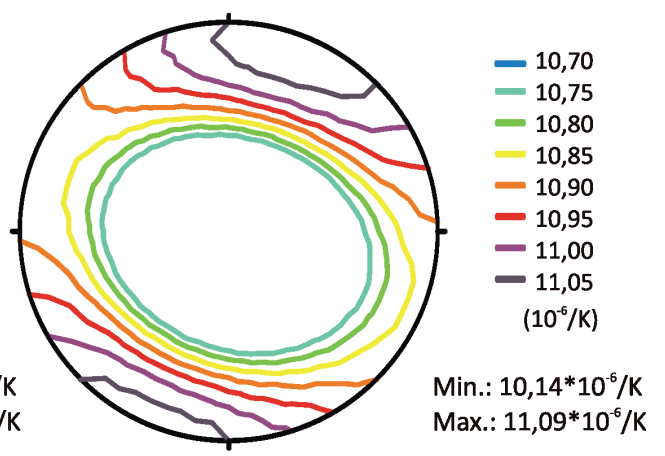

d)

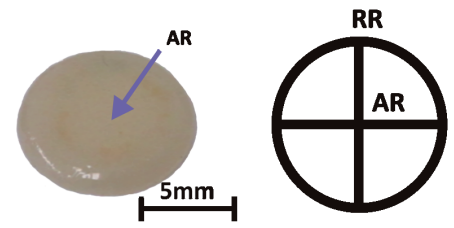

Abb. 80: Stereographische Projektion der thermischen Ausdehnung der $\mathrm{Li}_{2} \mathrm{Si}_{2} \mathrm{O}_{5}-\mathrm{Phase} \mathrm{im}$ Gusskanal (a) und in der Scheibe (b) für die Keramik IPS e.max Press. Messpunkte und Probenrichtungen (Axial- und Radialrichtung: AR und RR) innerhalb der stereographischen Projektion vom Gusskanal und von der verblendeten Scheibe sind in (c) bzw. (d) gegeben. Die Unterschiede in der anisotropen Ausdehnung sind durch unterschiedliche Texturen im Gusskanal und in der Scheibe bedingt (vgl. Abb. 61 und 62).

Für die $\mathrm{Li}_{2} \mathrm{Si}_{2} \mathrm{O}_{5}$-Phase wurden Literaturwerte für die thermische Ausdehnung von Mastelaro \& Zanotto (1999) verwendet (Einkristalldaten s. Anhang Tab. A.13), wobei in dieser Studie nur die Ausdehnungskoeffizienten einiger Kristallrichtungen angegeben wurden, aber nicht der vollständige Eigenschaftstensor. Aus den vorliegenden Daten war es möglich, die thermische Ausdehnung von $\mathrm{Li}_{2} \mathrm{Si}_{2} \mathrm{O}_{5}$ entlang der kristallographischen Achsen $a\left(d_{200} \rightarrow a_{11}\right), b\left(d_{020} \rightarrow a_{22}\right)$ und $c\left(d_{002} \rightarrow a_{33}\right)$ zu bestimmen, wobei die Aufstellung der monoklinen Elementarzelle berücksichtigt werden musste (hier $h k l$ und $a_{i j}$ in b1-Aufstellung; Transformation wie in Abschnitt 4.10 beschrieben). Der im monoklinen Kristallsystem erforderliche Term $a_{13}$ (b1-Aufstellung) bzw. $a_{12}$ (c1-Aufstellung) konnte jedoch nicht direkt aus den vorliegenden $d_{h k l}$ berechnet werden. Allerdings kann - in Näherung - der $a_{12}$-Term unter der Annahme wegfallen, dass sich die thermische Ausdehnung der $\mathrm{Li}_{2} \mathrm{Si}_{2} \mathrm{O}_{5}$-Phase nahezu orthorhombisch verhält, da auch die Kristallstruktur pseudo-orthorhomobisch ist $\left(\beta \approx 90^{\circ}\right)$ und die Atome nur sehr leichte Abweichungen von einer orthorhombischen Symmetrie zeigen (Liebau, 1961). Unter dieser Annahme variierte je nach Probe die thermische Ausdehnung für die $\mathrm{Li}_{2} \mathrm{Si}_{2} \mathrm{O}_{5}$-Phase richtungsabhängig zwischen $10,14 \cdot 10^{-6} / \mathrm{K}$ und 11, $09 \cdot 10^{-6} / \mathrm{K}$ (Abb. 80; $25-400^{\circ} \mathrm{C}$; Mastelaro \& Zanotto, 1999). Über den E-Modul dieser Phase lagen keine Einkristalldaten vor, sodass der makroskopische, anisotrope E-Modul für diese Phase nicht berechnet werden konnte.

\subsection{5 $\mathrm{Li}_{2} \mathrm{Si}_{2} \mathrm{O}_{5}$-Keramik IPS e.max CAD}

Die Hauptphase $\mathrm{Li}_{2} \mathrm{Si}_{2} \mathrm{O}_{5}$ zeigte in der Texturanalyse keine Vorzugsorientierung. Daher sind die physikalischen Eigenschaften dieser Keramik als isotrop anzunehmen. Wie im Fall der Keramik 
IPS e.max Press lagen nur Einkristalldaten für die thermische Ausdehnung vor (Mastelaro \& Zanotto, 1999). Der Wert für die isotrope thermische Ausdehnung der Hauptphase $\mathrm{Li}_{2} \mathrm{Si}_{2} \mathrm{O}_{5}$ liegt bei $10,7 \cdot 10^{-6} / \mathrm{K}\left(25-400^{\circ} \mathrm{C}\right)$.

\subsubsection{Feldspatkeramik Vitablocs - Esthetic Line}

Die makroskopischen, physikalischen Eigenschaften dieser Keramik ließen sich nicht direkt berechnen, aber es wurden jeweils für die einzelnen Mineralphasen die physikalischen Eigenschaften wie der E-Modul und die thermische Ausdehnung bestimmt. Hierbei ist zu berücksichtigen, dass die anisotropen, physikalischen Eigenschaften der einzelnen Phasen zwar theoretisch alleine betrachtet werden können, dass sie aber in der Praxis miteinander wechselwirken und dass sich die einzelnen Phasen gegenseitig in den makroskopischen, physikalischen Eigenschaften beeinflussen. Die physikalischen Eigenschaften der einzelnen Phasen können daher nur annäherungsweise Aufschluss über die makroskopischen, physikalischen Eigenschaften der Gesamtkeramik geben.

Im Fall der regellos angeordneten Nephelin-Phase sind auch ihre physikalischen Eigenschaften als isotrop anzunehmen. Unter Berücksichtigung der regellosen Textur ergab sich für die thermische Ausdehnung der Nephelin-Phase ein Wert von 6,97·10-6/K (Bonczar \& Barsch, 1975) und für den E-Modul ein Wert von 92 GPa (Einkristalldaten: Every \& McCurdy, 1992; Tab. A.14).

a)

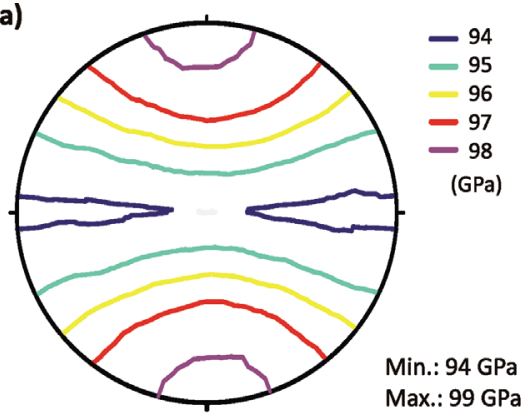

c)

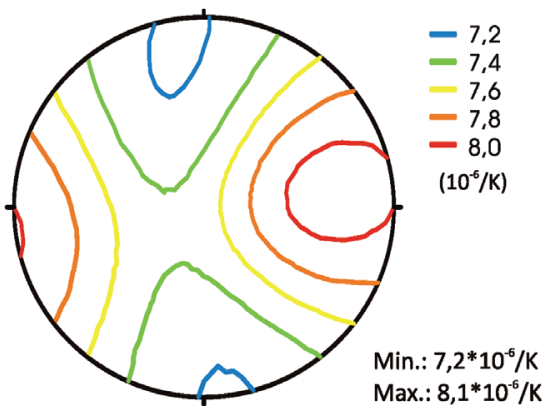

b)

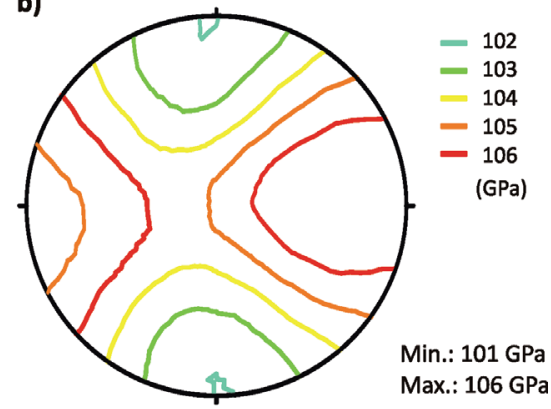

e)
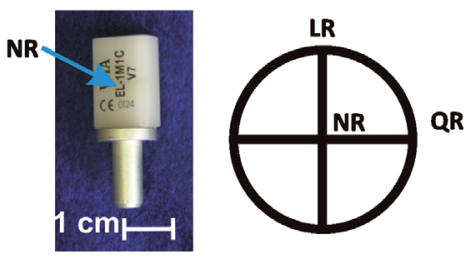

Abb. 81: Vitablocs - Esthetic Line: Stereographische Projektion des E-Moduls (in GPa) für die Phasen Mikroklin (a) und Sanidin (b) sowie die thermische Ausdehnung der Sanidin-Phase (c). In (d) sind der Messpunkt an der Probe und in (e) die Normal- (NR), Längs- (LR) und Querrichtung (QR) innerhalb der stereographischen Projektion dargestellt.

Für die Feldspatphasen Mikroklin und Sanidin liegen E-Modul-Einkristalldaten vor, wobei hier 
berücksichtigt werden muss, dass es sich bei Mikroklin und Sanidin um Mischkristalle handelt, deren stöchiometrische Zusammensetzung für die vorliegende Arbeit nicht sicher geklärt werden konnte. Als beste Näherung wurden Einkristalldaten von Ca-armen ( $\leq 2 \mathrm{Gew} \%$ ) bis Ca-freien Feldspäten verwendet (Haussühl, 1993; Every \& McCurdy, 1992; Tab. A.14). Insgesamt variierte der E-Modul zwischen 94-99 GPa innerhalb der Mikroklin-Phase und zwischen 106-113 GPa innerhalb der Sanidin-Phase (Abb. 81a und b). Die Berechnung der anisotropen thermischen Ausdehnung hingegen ist nicht ohne Weiteres durchzuführen. Thermische Ausdehnungsellipsoide mit ihrer Orientierung im Kristall wurden von Willaime et al. (1974) und Henderson (1979) für Plagioklase und Alkalifeldspäte ermittelt. Dabei stellte Henderson (1979) fest, dass die thermische Ausdehnung in monoklinen Alkalifeldspäten parallel zu $a_{b 1}=b_{c 1}$ am größten ist $\left(\alpha_{1} \approx 20 \cdot 10^{-6} \mathrm{~K}\right)$ und die zwei weiteren Terme der thermischen Ausdehnung sehr klein sind $\left(\alpha_{2} \approx \alpha_{3} \approx 1 \cdot 10^{-6} \mathrm{~K}\right.$; vgl. Tab. A.13), sodass das thermische Ausdehnungsellipsoid fast uniaxial erscheint und der isobare Volumenausdehnungskoeffizient $\alpha_{p}$ dem thermischen Ausdehnungskoeffizienten $\alpha_{1}$ entspricht. Annäherungsweise konnte der thermische Ausdehnungskoeffizient für die Sanidin-Phase nach Henderson (1979) berechnet werden (Abb. 81c).

\subsubsection{Glasinfiltrationskeramik Vita In-Ceram Alumina}

Die Kristallite aller Phasen sind regellos angeordnet. Daher erscheinen auch die physikalischen Eigenschaften - thermische Ausdehnung und E-Modul - dieser Keramik als isotrop. Im Mittel

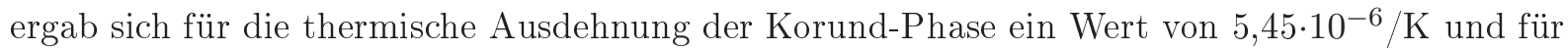
den E-Modul ein Wert von $474 \mathrm{GPa}$ (Einkristalldaten nach Shvyd'ko et al. (2002) und Ohno et al. (1986)).

\subsubsection{Glasinfiltrationskeramik Vita In-Ceram Zirkonia}

Die thermische Ausdehnung und der E-Modul der Korund-Phase zeigen insgesamt eine geringe Richtungsabhängigkeit in Abhängigkeit von den kristallographischen Richtungen (Abb. 82, Tab. A.13 und A.14). In der endgesinterten Probe variierten E-Modul und thermische Ausdehnung der Korund-Phase nur leicht richtungsabhängig (Abb. 83), während man für die tetragonale und monokline $\mathrm{Ce}-\mathrm{ZrO}_{2}$-Phase ein isotropes Verhalten annehmen kann. Die mittlere thermische Ausdehnung der $12 \mathrm{Mol} \%$ Ce-haltigen $\mathrm{ZrO}_{2}$-t-Phase liegt je nach betrachtetem Temperaturbereich bei $5,29 \cdot 10^{-6} / \mathrm{K}$ (Reddy et al., $1989 ; 25-125^{\circ} \mathrm{C}$ ) bzw. bei $\approx 11,5 \cdot 10^{-6} / \mathrm{K}$ (Scardi et al., 1992; 20$1000^{\circ} \mathrm{C}$ ) und ihr E-Modul bei ca. $262 \mathrm{GPa}$ (Kisi \& Howard, 1998). Für die monokline $\mathrm{ZrO}_{2}$-Phase liegen nur E-Modul-Werte für eine reine $\mathrm{ZrO}_{2}$-Phase ohne Ce-Zusatz vor (266 GPa für isotropes Verhalten; Chan et al., 1991). Dagegen sind für die thermische Ausdehnung nur Studien bei niedrigen Temperaturen von $10-300 \mathrm{~K}$ an reinem $\mathrm{ZrO}_{2}-\mathrm{m}$ vorhanden (Maistrelli et al., 1994). Der Wert für isotropes Verhalten in diesem niedrigen Temperaturbereich liegt bei $14,7 \cdot 10^{-6} / \mathrm{K}$. 
a)

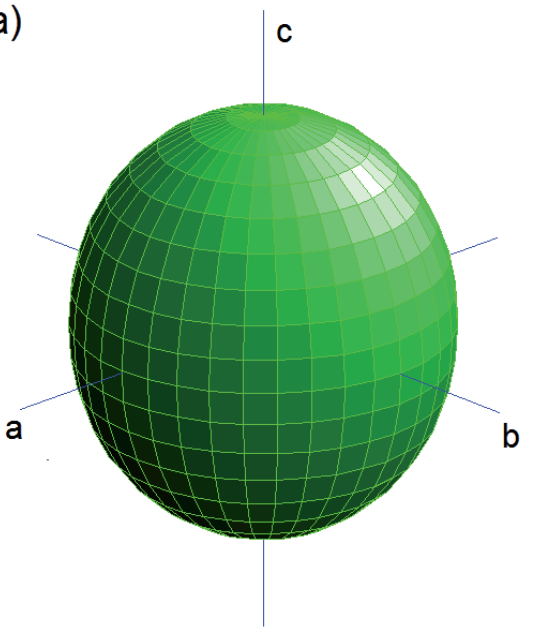

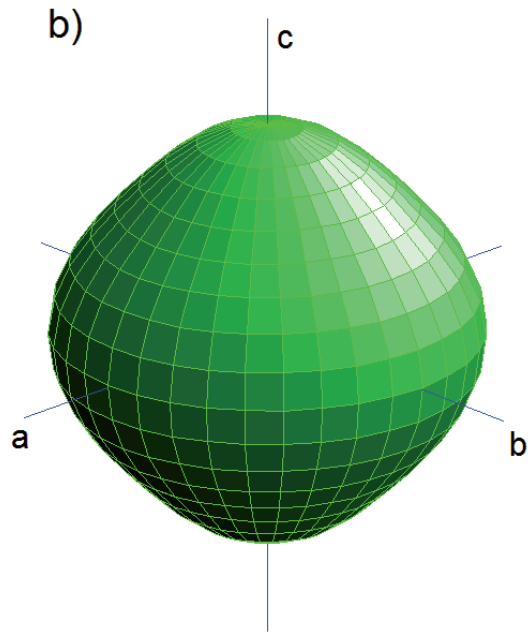

Abb. 82: Modellkörper für die Anisotropie der thermischen Ausdehnung (a) und des EModuls (b) der Korundphase. Die Modellkörper sind Rotationsellipsoide, die in unterschiedlichen kristallographischen Richtungen verschieden große Zahlenwerte annehmen. Im Fall der thermischen Ausdehnung und des E-Moduls werden die größten Zahlenwerte in der c-Richtung erreicht. Die niedrigsten Zahlenwerte liegen in der a- und b-Richtung vor. Einkristalleigenschaften nach Shvyd'ko et al. (2002) und Ohno et al. (1986).

a)

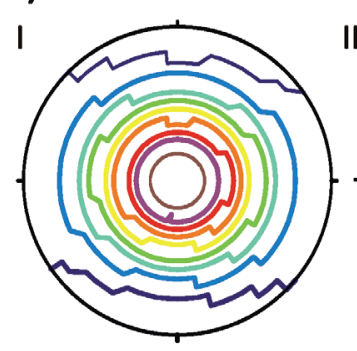

Min.: 5,41*10 $10^{-6} / \mathrm{K}$ Max.: $5,50^{*} 10^{-6} / \mathrm{K}$

c)

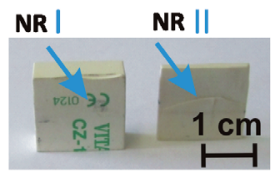

b)

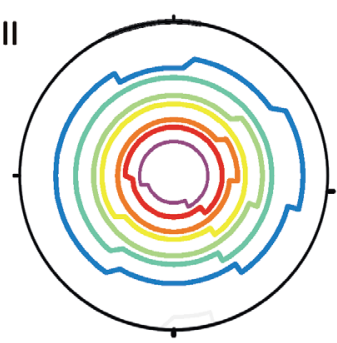

Min.: $5,42 * 10^{-6} / \mathrm{K}$ Max.: $5,49 * 10^{-6} / K$

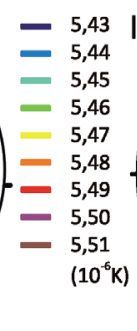

K)

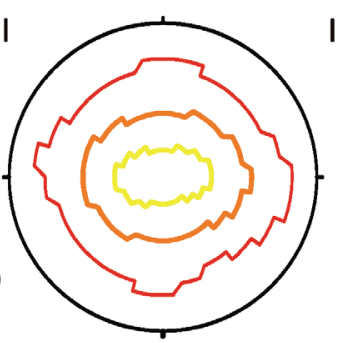

Min.: $471 \mathrm{GPa}$ Max.: $477 \mathrm{GPa}$

d)

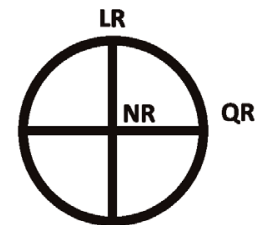

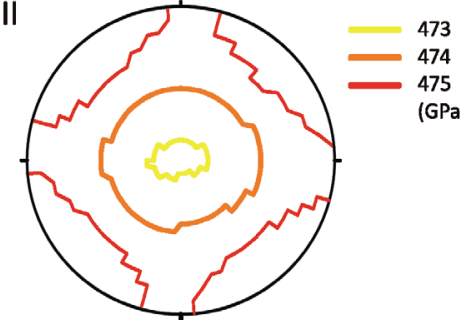

Min.: $472 \mathrm{GPa}$ Max.: $476 \mathrm{GPa}$

Abb. 83: Thermische Ausdehnung (a) und E-Modul (b) der Korund-Phase innerhalb der Gerüstkeramik Vita In-Ceram Zirkonia: Stereographische Projektionen vor (I) und nach (II) der Infiltration mit La-Glas. Einkristalldaten nach Shvyd'ko et al. (2002) und Ohno et al. (1986). (c) Messpunkte an vor- (I) und endgesinterter (II) Probe. (d) Lage der Normalrichtung (NR), Längsrichtung (LR) und Querrichtung (QR) innerhalb der stereographischen Projektion. 


\subsubsection{Goldlegierungen Degudent $U$ und Degulor $M$}

Da sich die Textur der Au-Hauptphase bei beiden Goldlegierungen nicht sicher bestimmen ließ und es sich bei dieser Phase um keine stöchiometrische Phase mit exakt bekannter chemischer Zusammensetzung handelte, wurden keine weiteren physikalischen Eigenschaften aus der Texturanalyse berechnet. Vom Hersteller liegen nur Angaben über die isotrope thermische Ausdehnung der fertigen Legierung Degudent $U$ vor, die in einem Temperaturbereich von $25-500^{\circ} \mathrm{C}$ einen Wert von $13,8 \cdot 10^{-6} / \mathrm{K}$ erreicht.

\subsubsection{Titan-Wurzelstift}
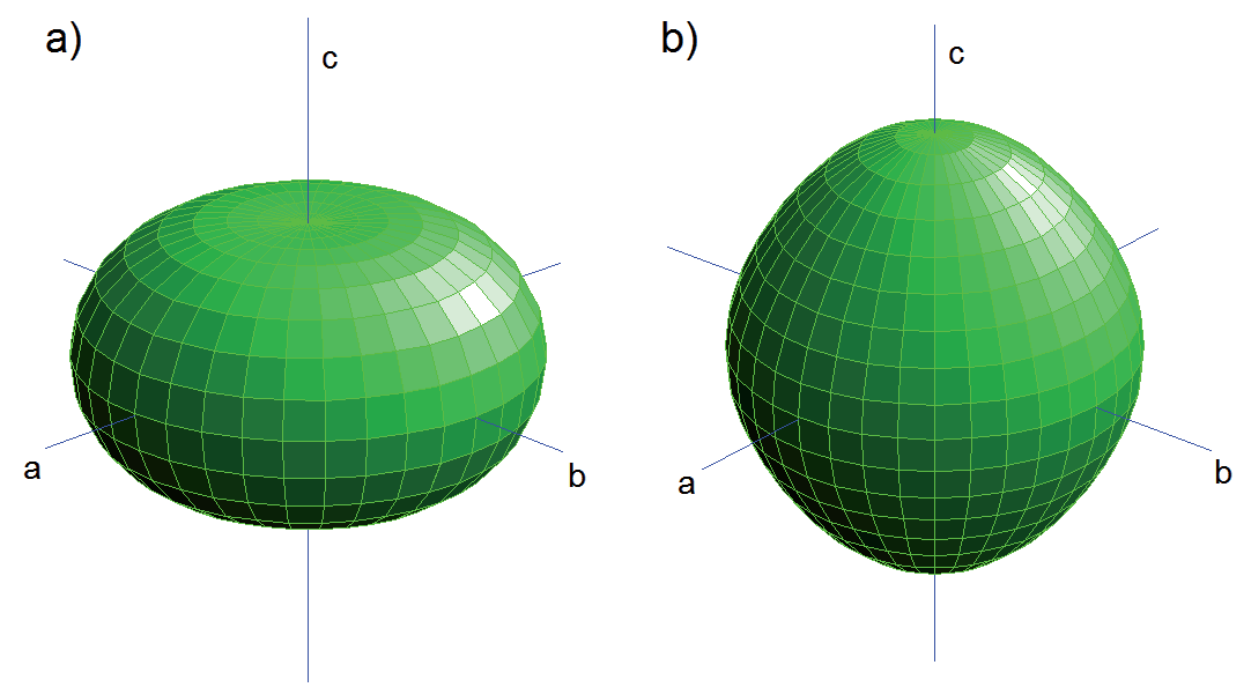

Abb. 84: Modellkörper für die Anisotropie der thermischen Ausdehnung (a) und des EModuls (b) von Titan. Die Modellkörper sind Rotationsellipsoide, die in unterschiedlichen kristallographischen Richtungen verschieden große Zahlenwerte annehmen. Im Fall der thermischen Ausdehnung wird in c-Richtung der kleinste Zahlenwert erreicht, während beim E-Modul in c-Richtung der größte Zahlenwert angenommen wird. Einkristalleigenschaften nach Pawar \& Deshpande (1968) und Every \& McCurdy (1992)

Die physikalischen Eigenschaften wie die thermische Ausdehnung und der E-Modul zeigen eine Richtungsabhängigkeit von den kristallographischen Achsen (Abb. 84, Tab. A.13 und A.14). Aus den Texturdaten (Synchrotronanalyse) mit Kombination der bekannten Einkristalleigenschaften (Pawar \& Deshpande, 1968; Every \& McCurdy, 1992) wurden die thermische Ausdehnung und der E-Modul für die Messpunkte I und II anisotrop berechnet. Für die makroskopischen, physikalischen Eigenschaften des Ti-Wurzelstiftes ergab sich nur eine leichte Anisotropie (Abb. 85). Der E-Modulwert variierte zwischen $160 \mathrm{GPa}$ und $167 \mathrm{GPa}$, wobei der geringste E-Modul in der Axialrichtung erzielt wird. Die thermische Ausdehnung hingegen nimmt in Axialrichtung mit $9,29 \cdot 10^{-6} / \mathrm{K}$ den größten Wert an. Die relativ geringe Anisotropie in den physikalischen Eigenschaften lässt sich dadurch erklären, dass die Anisotropien der Einkristalle durch die Textur gemittelt werden. 
a)

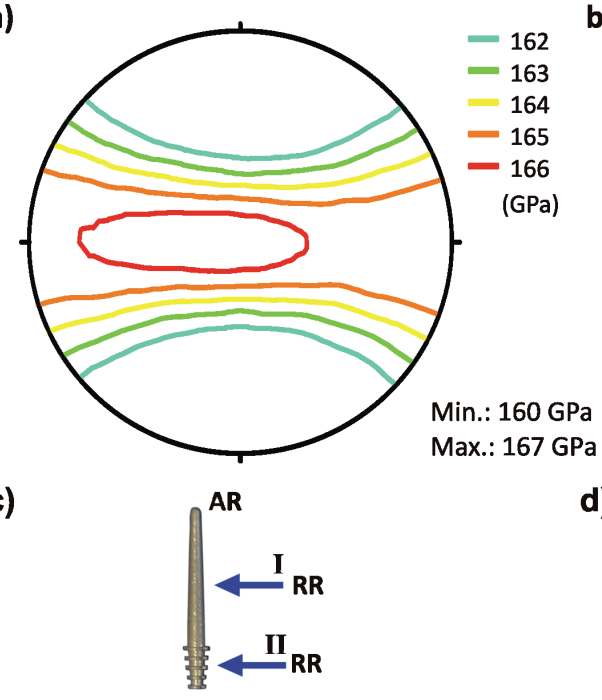

b)

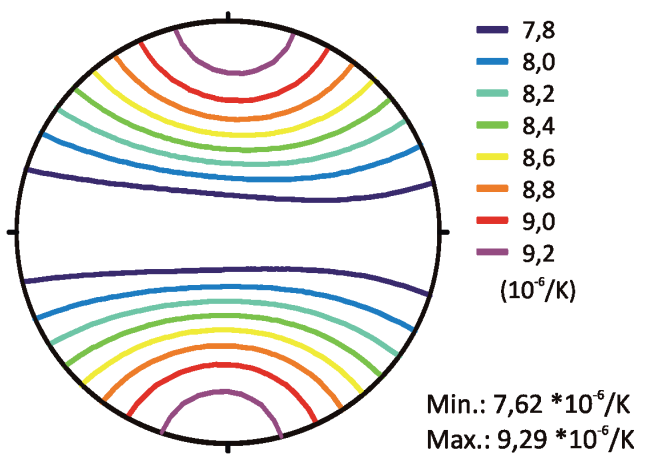

d)

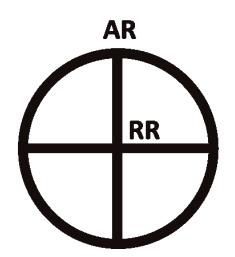

Abb. 85: Stereographische Projektionen des E-Moduls (a) und der thermischen Ausdehnung (b) für die Messung an der Probenmitte (Messpunkt I) des TitanWurzelstiftes der Firma Gebr. Brasseler. Die anisotropen Eigenschaften am Messpunkt II entsprechen in etwa denen am Messpunkt I (Thermische Ausdehnung: $7,56 \cdot 10^{-6} / \mathrm{K}-$ 9,31 $10^{-6} / \mathrm{K}$; E-Modul: $160 \mathrm{GPa}-167 \mathrm{GPa}$. (c) Probe mit den Messpunkten I und II. (d) Lage der Axial- (AR) und Radialrichtung (RR) innerhalb der stereographischen Projektion.

\subsubsection{Titan-Implantat OsseoSpeed}

Die anisotropen physikalischen Eigenschaften wurden für beide Messpunkte (I und II) aus der Texturanalyse in Kombination mit den bekannten Einkristalleigenschaften (Pawar \& Deshpande, 1968; Every \& McCurdy, 1992, Tab. A.13 und A.14) berechnet, wobei sich eine leichte Anisotropie in der thermischen Ausdehnung und des E-Moduls an beiden Messpunkten beobachten ließ (Bsp. für Messpunkt II in Abb. 86). Obwohl die Texturkomponenten des Titanwurzelstiftes und des Titan-Implantates sich zum Teil unterscheiden, sind die resultierenden anisotropen physikalischen Eigenschaften sehr ähnlich. Diese Ähnlichkeit ist in der Ähnlichkeit der Texturkomponenten (Ti-Wurzelstift: $g=(h k i l)\langle 10 \overline{1} 0\rangle$; Ti-Implantat: $g=(h k l)\langle 2 \overline{1} \overline{1} 0\rangle)$ und der Ausrichtung der Eigenschaftsellipsoide (gleiche Werte in $\langle 100\rangle$ und $\langle 010\rangle$ ) begründet. Wie beim Titanwurzelstift ist beim Titan-Implantat der E-Modul in Axialrichtung mit $159 \mathrm{GPa}$ am kleinsten und die

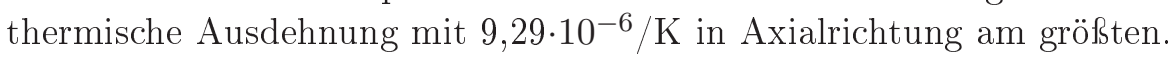


a)

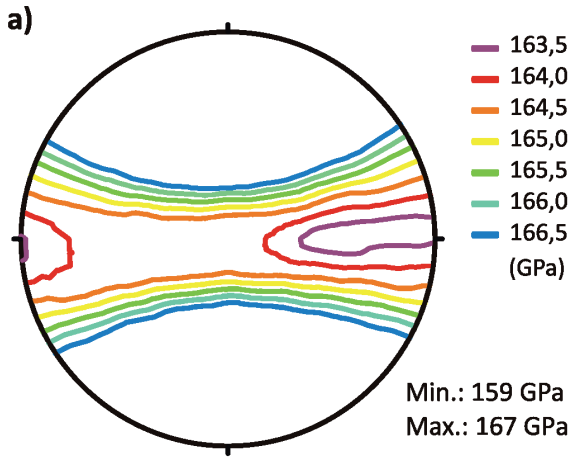

c)

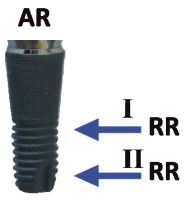

b)

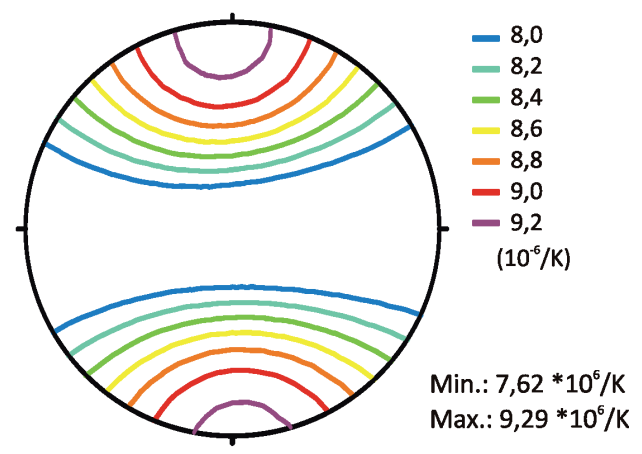

d)

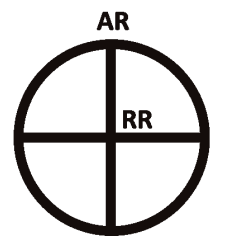

Abb. 86: Stereographische Projektionen des E-Moduls (a) und der thermischen Ausdehnung (b) für den Messpunkt II des Ti-Implantats OsseoSpeed. Die anisotropen Eigenschaften am Messpunkt I entsprechen in etwa denen am Messpunkt II (Thermische Ausdehnung: $7,59 \cdot 10^{-6} / \mathrm{K}-9,33 \cdot 10^{-6} / \mathrm{K}$; E-Modul: $160 \mathrm{GPa}-167 \mathrm{GPa}$ ). (c) Probe mit den Messpunkten I und II. (d) Lage der Axial- (AR) und Radialrichtung (RR) innerhalb der stereographischen Projektion. 


\subsection{Ergebnisse aus dem instrumentierten Eindringverfahren}

In Abb. 87 sind Beispiele von Härteeindrücken für die verschiedenen Dentalwerkstoffe zu sehen. Insgesamt lassen sich die Härteeindrücke in Metallen sehr gut erkennen, während sich die Eindrücke in Dentalkeramiken und Dentalkompositen wesentlich schlechter optisch erfassen lassen. Dieses stellt jedoch kein Problem in der Auswertung dar, da beim instrumentierten Eindringverfahren die physikalischen Größen direkt aus der Kraft-Eindringkurve ermittelt werden (vgl. Kap. 2.4). Für alle untersuchten Proben wurde die mittlere Härte nach Vickers sowie der mittlere reduzierte Eindringmodul ermittelt (Tab. 24). Aus dem reduzierten Eindringmodul wurde der E-Modul berechnet. Für diese Berechnung wurde $\nu_{\text {Indenter }}=0,07$ und $E_{\text {Indenter }}=1140 \mathrm{GPa}$ verwendet. Die Poissonzahl der Probe hingegen war meistens nicht genau bekannt, konnte aber aufgrund vorangegangener Untersuchungen eingegrenzt werden (s. Tab. 24).

Die mittleren Härtewerte variierten hauptsächlich in Abhängigkeit von der Werkstoffgruppe, aber auch nach ihrem Einsatzgebiet (Abb. 88, Tab. 24). Die niedrigsten Härtewerte wiesen die Prothesenkunststoffe mit 23-28 HV 0,01 sowie der provisorische Kunststoff Clip mit 13 HV 0,01 auf. Danach folgen die übrigen Dentalkomposite, wobei hier größere Unterschiede in den Härtewerten (44-149 HV 0,01) zu sehen sind. Die großen Unterschiede sind vom Hersteller teilweise beabsichtigt, da z.B. die Komposite Luxacore und Rebilda LC und DC (46 HV 0,01 und 83 HV 0,01) für den Stumpfaufbau mit Kontakt zum relativ weichen Dentin gedacht sind, während andere Komposite wie Tetric und Ceram X (107 HV 0,01 und 85 HV 0,01) für Kavitäten mit Kontakt zum härteren Zahnschmelz eingesetzt werden und Cimara Zircon (149 HV 0,01) auch zur Ausbesserung von Dentalkeramiken verwendet wird. Der Glasionomerzement Ketac Fil Plus wies mit 159 HV 0,1 eine ähnliche Härte wie das Komposit Cimara Zirkon auf.

Eine im Verhältnis zu den Dentalkompositen erhöhte Härte zeigten die Dentalmetalle und -legierungen (HV: 191-626), von denen die meisten Werte um 300 HV 0,03 aufwiesen. Am weichesten ist das Amalgam Amalcap (HV: 191) und am härtesten die Co-Cr-Legierung Remanium GM 800+ (HV: 626), wobei die zuletzt genannte Legierung nicht für Inlays oder Kronen, sondern nur für Modellgussprothesen verwendet wird.

Die härtesten Zahnersatzmaterialien bilden die Dentalkeramiken. Ihre Härtewerte liegen um 700 HV 0,03 und im Fall der La-glasinfiltrierten Keramiken sogar bei 1300 HV 0,03.

Auch die elastischen Eindring-Module $E$ zeigen einen ähnlichen Trend: Die niedrigsten $E$-Werte zeigen die Prothesenkunststoffe (ca. 3,5 GPa), auf die die Dentalkomposite mit 4-18 GPa folgen, wobei Clip als Provisorium mit nur 0,12 GPa eine Ausnahme bildet. Einen leicht höheren E-Modul zeigte das GIZ Ketac Fil Plus (26 GPa). Danach kommen die Dentalkeramiken und Dentalmetalle.

Relativ niedrige E-Werte haben die Keramiken der Vitablocs-Serie (ca. $62 \mathrm{GPa}$ ), die Goldlegierung Degulor $M$ (51 GPa) und das Amalgam Amalcap regular (68 GPa). Etwas höher liegen die E-Module der Goldlegierung Degudent $U$ (86 GPa) und des Titanwurzelstiftes (82 GPa). Die LS2-Keramiken wiesen im Endzustand E-Wert von ca. $97 \mathrm{GPa}$ auf. Davon heben sich deutlich die federelastischen NEM-Legierungen Wiron 99 und Remanium GM 800+ (161 GPa; $206 \mathrm{GPa})$ sowie die Keramiken Cercon base ( $\approx 195 \mathrm{GPa})$, Vita In-Ceram Alumina (289 GPa) und Zirkonia ab (231 GPa). 

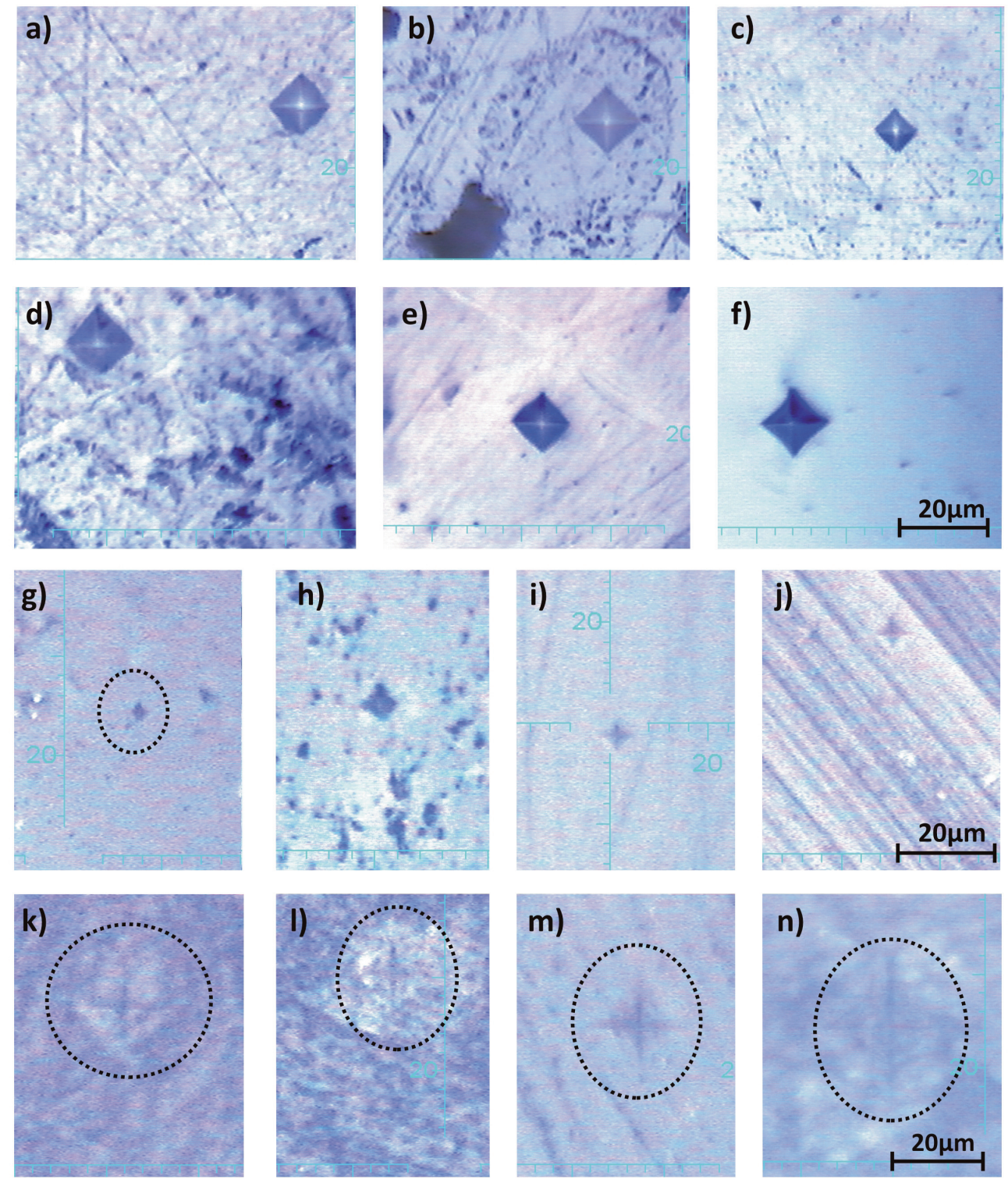

Abb. 87: Auflichtaufnahmen von Härteeindrücken ausgewählter Dentalwerkstoffe.

Dentallegierungen und Dentalmetalle (Prüflast: $300 \mathrm{mN}$ ): (a) Ti-Wurzelstift, (b) Amalcap regular, (c) Remanium GM 800+, (d) Degudent U, (e) Degulor $M$ und (f) Wiron99.

Dentalkeramiken (Prüflast: $300 \mathrm{mN}$ ): (g) Vitablocs A.3, (h) Vita In-Ceram Alumina, (i) IPS e.max Press im fertigen Zustand und (j) IPS e.max CAD.

Dentalkomposite, Prothesenkunststoffe und Glasionomerzemente (Prüflast: $100 \mathrm{mN}$, für GIZ 1N): (k) Tetric Evo Flow, (l) Venus C3, (m) Bioplus Dentin-Bereich und (n) das GIZ Ketac Fil Plus. 
Tab. 24: Ergebnisse aus dem instrumentierten Eindringverfahren: Vickershärte HV, reduzierter Eindringmodul $E_{r}$ und elastischer E-Modul $E . E_{r}$ und in $E$ sind in GPa angegeben; $n$ steht für die Anzahl der Einzelmessungen; Standardabweichungen sind in Klammern angegeben.

\begin{tabular}{|c|c|c|c|c|c|}
\hline Materialtyp & Handelsname & $\mathrm{HV}(\mathrm{MW})$ & $E_{r}$ & $E$ & $n$ \\
\hline \multirow{10}{*}{ Dentalkomposite $\mathrm{e}^{1,2}$} & Tetric & $107(19)$ & $17(2)$ & 15,4 & 12 \\
\hline & Tetric Evo Flow & $44(4)$ & $6,8(0,3)$ & 6,1 & 11 \\
\hline & Tetric Evo Ceram & $63(6)$ & $9,3(0,8)$ & 8,4 & 10 \\
\hline & Luxacore & $46(13)$ & $4,2(0,8)$ & 3,8 & 11 \\
\hline & Rebilda $L C$ & $83(11)$ & $14(1)$ & 12,6 & 12 \\
\hline & Rebilda $D C$ & $71(11)$ & $9(1)$ & 8,1 & 11 \\
\hline & Clip & $1,3(0,6)$ & $0,14(0,03)$ & 0,12 & 10 \\
\hline & Ceram X & $85(24)$ & $14(3)$ & 12,6 & 11 \\
\hline & Venus & $85(5)$ & $13,0(4)$ & 11,7 & 11 \\
\hline & Cimara Zircon & $149(34)$ & $20(3)$ & 18,1 & 11 \\
\hline \multirow[t]{2}{*}{ Glasionomerzement $^{3}$} & Ketac Fil Plus $(100 \mathrm{mN})$ & $343(220)$ & $42(13)$ & 39 & 12 \\
\hline & Ketac Fil Plus $(1 \mathrm{~N})$ & $159(49)$ & $28(4)$ & 26 & 11 \\
\hline \multicolumn{2}{|c|}{ Prothesenkunststoffe ${ }^{1,2}$ Pala-Press } & $23(4)$ & $3,8(0,3)$ & 3,4 & 19 \\
\hline & Weithur-Press & $27(3)$ & $4,2(0,2)$ & 3,8 & 12 \\
\hline & Aesthetic (normaler Fasernanteil) & $25(3)$ & $3,9(0,3)$ & 3,5 & 12 \\
\hline & Aesthetic (erhöhter Fasernanteil) & $24(3)$ & $3,8(0,3)$ & 3,4 & 14 \\
\hline & Bioplus (gesamt) & $28(1)$ & $4,0(0,7)$ & 3,6 & 32 \\
\hline \multirow[t]{8}{*}{ Keramiken ${ }^{4,5}$} & Cercon base & $993(324)$ & $175(27)$ & $193-198$ & 8 \\
\hline & Vitablocs Mark II - A 3.5 & $661(77)$ & $63(6)$ & $62-64$ & 12 \\
\hline & Vitablocs EL & $662(58$ & $61(3)$ & $60-62$ & 20 \\
\hline & Vita In-Ceram Alumina & $1374(140)$ & $243(34)$ & 289 & 20 \\
\hline & Vita In-Ceram Zirconia & $1203(281)$ & $204(36)$ & 231 & 11 \\
\hline & IPS e.max Press (Rohling) & $780(50)$ & $102(5)$ & 104-107 & 18 \\
\hline & IPS e.max Press (Scheibe) & $733(41)$ & $94(3)$ & $95-98$ & 11 \\
\hline & $I P S$ e.max $C A D$ (endgesintert) & $695(84)$ & $94(6)$ & $95-98$ & 17 \\
\hline Metalle und & Amalcap regular & $191(48)$ & $73(7)$ & 68 & 20 \\
\hline \multirow{5}{*}{ Legierungen $^{4,6}$} & Titan-Stift & $309(12)$ & $92(2)$ & 90 & 10 \\
\hline & Degudent $U$ & $272(26)$ & $86(6)$ & 76 & 11 \\
\hline & Degulor $M$ & $330(34)$ & $59(3)$ & 51 & 12 \\
\hline & Wiron 99 & $264(25)$ & 191(10) & 206 & 16 \\
\hline & Remanium $800+$ & $582(89)$ & $156(45)$ & 161 & 18 \\
\hline
\end{tabular}

${ }^{1}[\mathrm{HV} 0,01 / 20]$

${ }^{2}$ Berechnung von $E$ mit der Poissonzahl $\nu_{\text {Dentalkomposit }} \approx \nu_{P M M A} \approx 0,33$ (Sakaguchi \& Powers, 2012; Greaves et al., 2011)

${ }^{3}$ Probe sehr heterogen, daher zwei verschiedene Prüflasten gewählt: $100 \mathrm{mN}$ und $1 \mathrm{~N}$ [HV 0,01/20 und HV $0,1 / 20]$

${ }^{4}$ [HV 0,03/20]

${ }^{5}$ Berechnung von $E$ mit der Poissonzahl 0,21< $\nu_{\text {Dentalkeramik }}<0,26$ (Sakaguchi \& Powers, 2012); für die Vita In-Ceram-Keramiken liegen genauere Werte vor: $\nu_{\text {Alumina }}=0,25$ und $\nu_{\text {Zirkonia }}=0,26$ (Guazzato et al., 2002)

${ }^{6}$ Berechnung von $E$ mit $\nu_{\text {Amalgam }}=0,36$ (Grenoble \& Katz, 1971), $\nu_{T i}=0,322$ (Fisher \& Manghani, 1971), $\nu_{\text {Goldlegierung }}=0,43$ (Kumar et al., 2003), $\nu_{C o-29 C r-6 M o}=0,3$ (Kumagai et al., 2005) und $\nu_{N i-C r-M o}=0,28$ (Käse \& Tesk, 1989) 


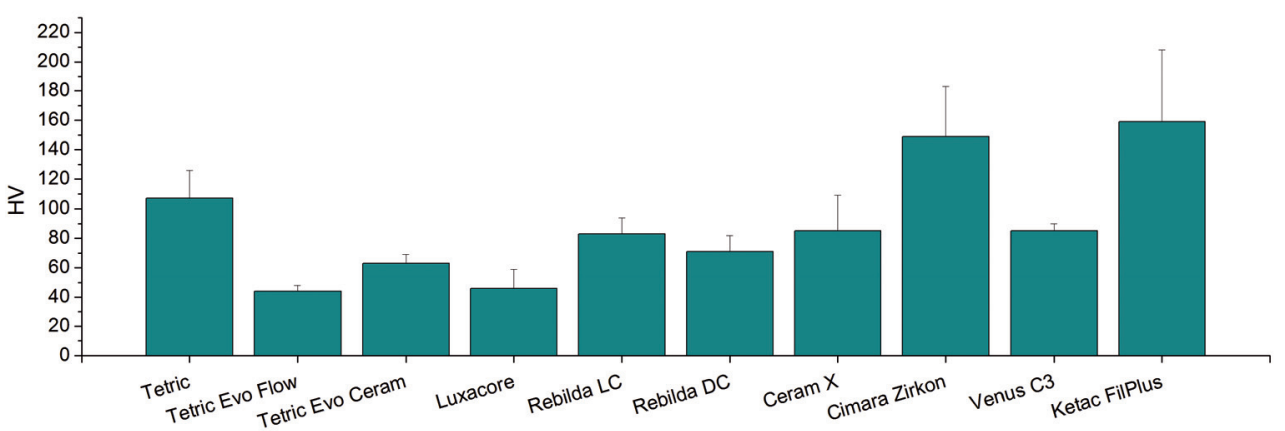

(a) Dentalkompsite und Glasionomerzement.

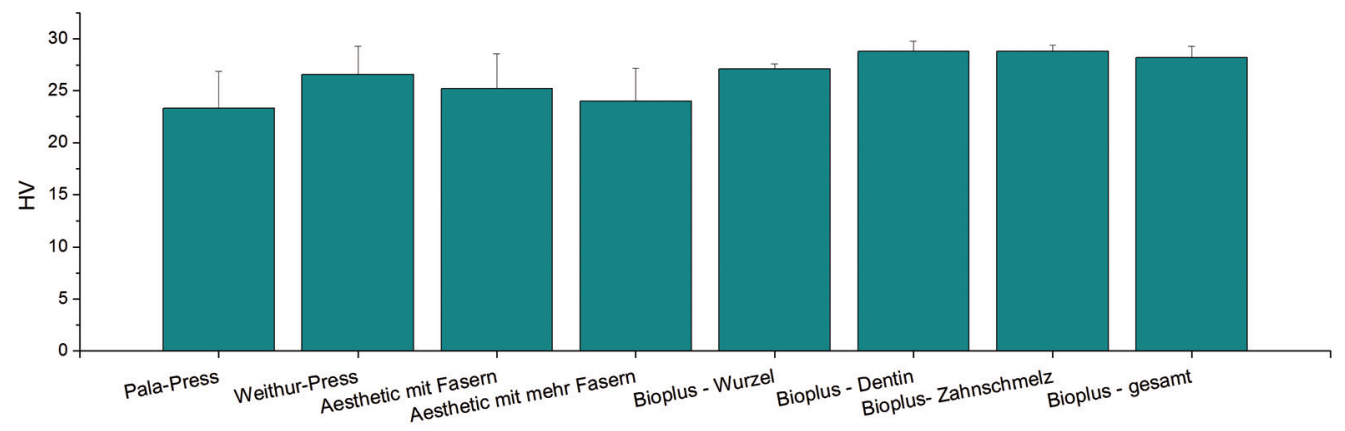

(b) Prothesenkunststoffe.

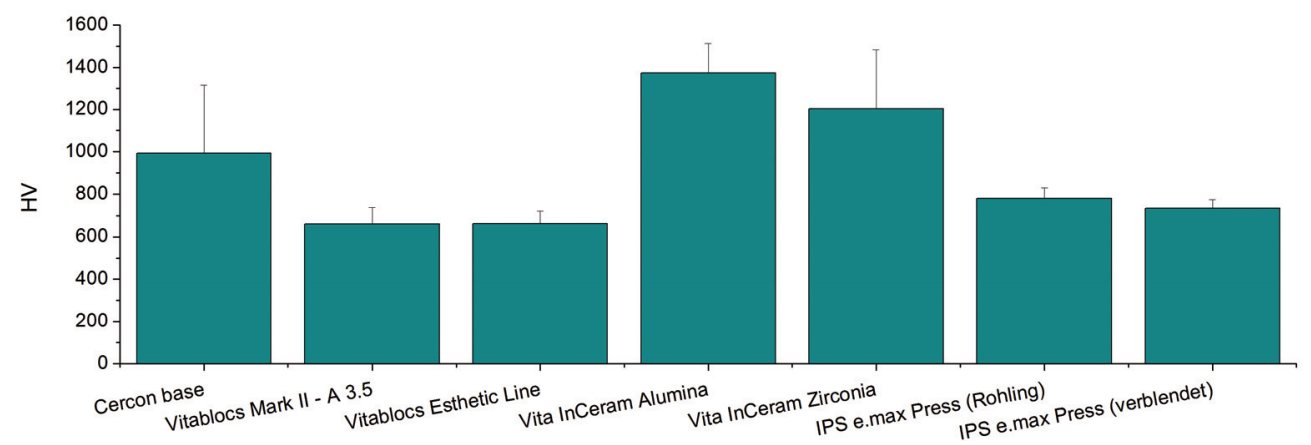

(c) Dentalkeramiken.

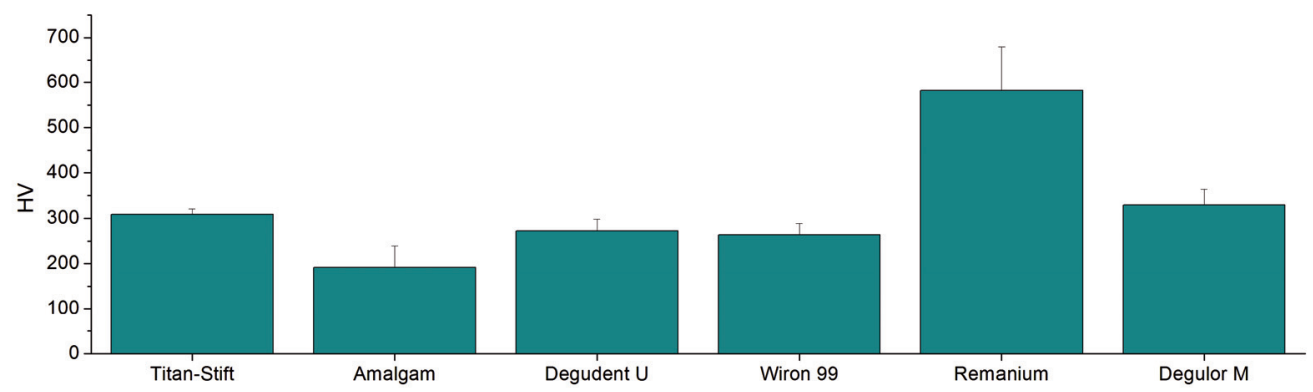

(d) Dentalmetalle und -legierungen.

Abb. 88: Graphische Darstellung der Vickershärte nach Werkstoffgruppen . 


\section{Fehlerbetrachtung}

\subsection{Fehlerquellen bei REM-Untersuchungen}

Eine mögliche Fehlerquelle der standardfreien EDX-Analysen ist, dass nur rein rechnerisch ermittelte Korrekturfaktoren für die quantitative Elementanalyse verwendet werden. Genauere Ergebnisse können durch Kalibration der zu untersuchenden Elemente an einem geeigneten Standard erzielt werden, wobei der Standard am besten eine ähnliche chemische Zusammensetzung wie die Probe aufweisen sollte. Um eine erste Übersicht über die Proben und ihre Beschaffenheit zu erhalten, ist die standardfreie EDX-Analyse jedoch bestens geeignet.

Weitere Fehlerquellen der EDX-Analysen liegen in der Probenbeschaffenheit: zum einen in der Oberflächenbeschaffenheit (Rauigkeit) und zum anderen in den vorhandenen Elementen, deren Röntgenlinien z.T. stark überlappen können (Bsp.: Platingruppenelemente). Außerdem kann die Korngrößenverteilung problematisch sein, wenn die zu untersuchenden Körner kleiner als der fokussierte Strahl sind.

\subsection{Fehlerquellen bei Untersuchungen an der Elektronenstrahlmikrosonde}

Elementanalysen mit einem wellenlängen-dispersiven System sind in der Regel genauer als standardfreie EDX-Analysen, stellen aber auch größere Anforderungen an die zu untersuchende Probe. Die Probe muss eine glatte Oberfläche haben, die planar - senkrecht zum Elektronenstrahl - eingebaut ist. Dafür kann eine höhere Aufösung der Röntgenlinien erreicht werden, sodass Gewichtsanteile von Elementen mit ähnlicher Ordnungszahl genauer bestimmt werden können. Allerdings sind wie bei der EDX-Analyse die Platingruppenelemente problematisch, da ihre K-Linien sehr dicht beieinander liegen. Das Problem konnte hier größtenteils unter Verwendung der L-Linien gelöst werden, wobei es auch zwischen den L-Linien dieser Elemente zu leichten Überlappungen kam. Diese Überlappungen konnten aber mit Korrekturfaktoren herausgerechnet werden.

Außerdem ist eine zu geringe Korngröße der zu untersuchenden Mineralphase problematisch, da diese bei zu großem Elektronenstrahl zu Mischanalysen führt. Durch Vergleich der Mischanalysen mit Analysen an reinen Mineralen kann aber auf die tatsächliche Zusammensetzung des gemessenen Korns weitestgehend zurückgeschlossen werden. Bei kleineren Körnern treten zusätzlich auch Korngrenzeneffekte auf, wobei zum Korngrenzeneffekt auch Reliefeffekte beitragen: Bei gleicher Präparation zweier Mineralphasen kommt es aufgrund ihrer unterschiedlichen Härtegrade zu einem leicht unterschiedlichen Abtrag, sodass eine Mineralphase ein höheres Relief als ein benachbartes Korn einer anderen Mineralphase aufweist. Solche Reliefunterschiede können dazu führen, dass je nach Mineralphase mehr (positives Relief) oder weniger (negatives Relief) Elektronen wieder aus der Probe austreten und detektiert werden. 


\subsection{Fehlerquellen bei der röntgenographischen Phasenanalyse}

Unter der Annahme, dass die Messungen korrekt durchgeführt werden (d.h. repräsentative Probe, Kalibrierung der Wellenlänge mit einem NIST-Standard, korrekte Bestimmung des Strahlmittelpunktes auf dem Detektor), liegen die wichtigsten Fehlerquellen bei der Auswertung der Synchrotrondaten mit der röntgenographischen Phasenanalyse in der Wahl der Kristallstrukturdaten mit angemessenen atomaren Auslenkungsparametern sowie in der Wahl sinnvoller Profilparameter. Auch das Setzen eines sinnvollen Untergrundes kann in einigen Fällen (z.B. komplexer Untergrund durch Röntgenstreuung an amorphem Glas) problematisch werden. Daneben führen Mineralphasen mit niedrig symmetrischer Kristallstruktur zu (starken) Reflexüberlappungen und erschweren die Phasenanalyse sehr, besonders wenn sich die zu untersuchenden Phasen nur geringfügig unterscheiden (Bsp.: Feldspat-Gruppe).

\subsection{Fehlerquellen bei den Texturanalysen und der Berechnung der physikalischen Eigenschaften}

Wie bei der röntgenographischen Phasenanalyse liefern gute röntgenographische Messungen eine gute Analysebasis, wobei bei den konventionellen Texturmessungen zusätzlich berücksichtigt werden muss, dass mit steigendem Kippwinkel $\chi$ der Röntgenstrahl defokussiert wird und die mit einem Punktzähler detektierbaren Intensitäten deutlich abnehmen. Dieser Effekt ist besonders ab einem Kippwinkel von $\chi=70^{\circ}$ bemerkbar, sodass die konventionellen Polfigurmessungen nur bis zu diesem Kippwinkel vorgenommen wurden. Darunter ist die Defokussierung vernachlässigbar klein bzw. mittelt sich bei der Berechnung der ODF heraus. Eine weitere Fehlerquelle liegt in der Grobkörnigkeit der Probe, da bei einer grobkörnigen Probe die Gefahr besteht, dass die Kornstatistik für die Texturanalyse nicht ausreichend ist. Außerdem muss eine ausreichende Zählstatistik vorhanden sein.

In den Synchrotron-Transmissionsaufnahmen ist die Defokussierung kein Problem und auch die Zählstatistik ist in der Regel besser. Allerdings können andere Effekte auftreten: z.B. ist der Weg des Synchrotronstrahls durch eine Probe mit Quader-Geometrie bei unterschiedlichem Rotationswinkel $\omega$ verschieden lang, wobei es zu verstärkter Reflexion, aber auch Absorption kommt. Daher wurden nur im Fall von zylindrischen Proben Texturmessungen in einem Bereich von $-90^{\circ} \leq \omega \leq+90^{\circ}$ vorgenommen, während für quaderförmige Proben ein Winkelbereich von $-80^{\circ} \leq \omega \leq+80^{\circ}$ gewählt wurde. Der Winkelbereich $-80^{\circ} \leq \omega \leq+80^{\circ}$ reichte in der Regel für eine gute quantitative Texturanalyse aus.

Nach korrekt durchgeführten Texturmessungen liegen die wichtigsten Fehlerquellen der kombinierten Rietveld-Texturanalyse - wie bei der röntgenographischen Phasenanalyse - in der Wahl der Kristallstrukturdaten, in der sinnvollen Verwendung geeigneter Profilparameter und in einer guten Untergrundanpassung. Außerdem kann die ODF-Auflösung individuell gewählt werden, wobei bei scharfen Texturen mit einer geringeren Streubreite in der Poldichteverteilung eine höhere Auflösung nötig ist (hier: $3,75^{\circ}$ bzw. $5^{\circ}$ ausreichend für E-WIMV-Methode). Proben mit mehreren Mineralphasen (besonders mit niedrig symmetrischer Kristallstruktur) zeigen im Beugungsbild meist Reflexüberlappungen und sind insgesamt schwieriger auszuwerten, wobei die Qualität der Anpassung nicht nur am GOF- und R-Wert gemessen werden kann, sondern auch 
immer durch den Vergleich von experimentellen mit berechneten Polfiguren überprüft werden sollte. In den hier vorliegenden Untersuchungen wurde die Güte der Texturanpassung für jede Untersuchung sowohl rechnerisch als auch durch Vergleich der experimentellen mit den berechneten Polfiguren kontrolliert.

\subsection{Fehlerquellen beim instrumentierten Eindringverfahren zur Bestimmung der Vickershärte und des E-Moduls}

Einige Fehlerquellen der Härteprüfung lassen sich durch sorgfältige Probenpräparation minimieren (z.B. Oberflächenrauigkeit und Planarität), wobei die Oberflächenrauigkeit durch die Wahl der Schleif- und Politurmittel nur bis zu einem gewissen Grad reduziert werden kann. Nicht kontrollierbare Fehlerquellen liegen in der vorgegebenen Probenbeschaffenheit wie chemische und mineralogische Heterogenität sowie der Korngrößenverteilung. Durch eine erhöhte Anzahl an Messungen pro Probe $(N \geq 10)$ wurden repräsentative Durchschnittswerte für die Gesamteigenschaften einer Probe ermittelt. Im Fall des Glasioniomerzementes Ketac Fil Plus wurde aufgrund der starken Inhomogenität der Füllpartikelart und -größe zusätzlich die Prüflast auf $1 \mathrm{~N}$ erhöht, sodass insgesamt pro Härteeindruck eine größere Fläche mit mehr Füllpartikeln gemessen werden konnte. 


\section{Diskussion}

\subsection{Anwendbarkeit der röntgenographischen Glaskonzentrationsbestimmung und Vergleich der Ergebnisse mit Herstellerangaben}

Zur röntgenographischen Bestimmung des Glasgehaltes wurden zwei verschiedene Methoden verwendet (vgl. dazu die Abschnitte 2.2.1, 4.2 und 5.5):

- Rietveld-Analyse mit ZnO-Pulver als interner Standard (IS-Methode)

- Modellierung der Glasphase durch eine scheinkristalline Struktur (GSM-Methode)

Die Anwendbarkeit beider Methoden wurde zunächst an Modellproben (A, B, C, B1) getestet und dann auf die vorliegenden Dentalkeramiken übertragen. Beide Methoden ließen sich mit einer Genauigkeit von ca. 5 Gew.\% anwenden, wobei hier tendenziell die IS-Methode bei kleineren Glasgehalten und die GSM-Methode bei größeren Glasgehalten genauere Werte erzielten (Tab. 19 und 20). Kritische Punkte bei der Rietveldverfeinerung nach der IS-Methode ist die Verwendung guter Kristallstrukturen sowie eine gute Anpassung des Untergrunds. Probleme bei der IS-Methode können auftreten, wenn die durchschnittliche atomare Masse und somit die Röntgenstreuung und -absorption von Glas, Mineralphasen und internem Standard sehr unterschiedlich sind. Bei der GSM-Methode dagegen ist ein besonders kritischer Punkt die Wahl eines geeigneten Strukturmodells für die Glasphase.

Außerdem wurden im Rahmen dieser Arbeit zwei verschiedene Behältermaterialien getestet: (1) Reinst-Al-Röhrchen $\left(\varnothing_{\text {Innen }}=6,7 \mathrm{~mm}\right)$ und $(2)$ PI-Kapillare $\left(\varnothing_{\text {Innen }} \approx 1,7 \mathrm{~mm}\right)$. Insgesamt konnte aus den vorliegenden Messungen kein wesentlicher Einfluss des Behältermaterials auf die Genauigkeit in der Bestimmung des Glasgehaltes festgestellt werden, wobei jedoch zu berücksichtigen ist, dass die Anzahl an Experimenten nicht repräsentativ (siehe Kap. 5.5 Tab. 20) und auch das Beugungsvolumen unterschiedlich groß war.

\section{Glasgehalte der Dentalkeramiken}

Im Fall der Vita In-Ceram-Keramiken liegen gemäß Hersteller genaue Angaben über die Glasgehalte vor (Pröbster \& Groten, 2008). Bei beiden Keramiken wurde nach der IS-Methode ein zu hoher Glasanteil berechnet (Tab. 25; GSM-Methode war nicht anwendbar). Diese Unterschiede könnten dadurch zu erklären sein, dass entweder der Glasanteil bei den hier untersuchten Proben tatsächlich höher war oder aber dass die Unterschiede in der atomaren Masse zwischen den kristallinen Phasen (Korund, Zirkondioxid und Zinkit) und dem La-Al-Si-Glas zu groß waren. In diesem Fall ist letzteres am wahrscheinlichsten, da das im Glas enthaltene Lanthan eine große atomare Masse besitzt und somit - im Gegensatz zu Korund - auch ein anderes Absorptionsverhalten und Streuungsvermögen aufweist.

Für die Dentalkeramik Vitablocs Esthetic Line liegen keine Angaben über ihren Glasgehalt vor, aber für die Feldspat-Keramik Vitablocs Mark II wird vom Hersteller ein Glasgehalt von ca. 70 Vol\% angenommen. Dieser Wert kann als Vergleichswert für Vitablocs Esthetic Line dienen, da diese Dentalkeramiken eine sehr ähnliche chemische und mineralogische Zusammensetzung 
Tab. 25: Übersicht über Glasgehalte und Mineralphasenanteile der hier untersuchten GlasDentalkeramiken.

\begin{tabular}{|c|c|c|c|c|}
\hline & \multicolumn{2}{|c|}{ Glasgehalte } & \multicolumn{2}{|c|}{ Mineralphasengehalte } \\
\hline & $\begin{array}{l}\text { Phasenanalyse } \\
\text { (Gew.\%) }\end{array}$ & Hersteller & Phasenanalyse (Gew.\%) & $\begin{array}{c}\text { Hersteller } \\
\text { (Gew.\%) }\end{array}$ \\
\hline In-Ceram Alumina & 38 & 25 Gew. $\%$ & $100 \mathrm{Al}_{2} \mathrm{O}_{3}$ & $100 \mathrm{Al}_{2} \mathrm{O}_{3}$ \\
\hline In-Ceram Zirconia & 32 & 20 Gew.\% & $\begin{array}{c}68 \mathrm{Al}_{2} \mathrm{O}_{3}, 30 \mathrm{ZrO}_{2} \text {-t } \\
3 \mathrm{ZrO}_{2}-\mathrm{m}\end{array}$ & $\begin{array}{c}67 \mathrm{Al}_{2} \mathrm{O}_{3}, 33 \\
\mathrm{ZrO}_{2} \text {-t }\end{array}$ \\
\hline IPS e.max Press & $25-53$ & $\begin{array}{c}30- \\
40 \mathrm{Vol} . \%^{1}\end{array}$ & $\begin{array}{c}82 \mathrm{Li}_{2} \mathrm{Si}_{2} \mathrm{O}_{5}, 10 \mathrm{Li}_{3} \mathrm{PO}_{4} \\
6 \mathrm{Li}_{2} \mathrm{SiO}_{3}, 3 \mathrm{Li}_{2} \mathrm{ZnSiO}_{4} \\
\end{array}$ & $\begin{array}{l}\mathrm{Li}_{2} \mathrm{Si}_{2} \mathrm{O}_{5} \\
\mathrm{Li}_{3} \mathrm{PO}_{4} \\
\end{array}$ \\
\hline Vitablocs EL & $\approx 88$ & $\begin{array}{c}>70 \\
\text { Vol\% } \%^{2}\end{array}$ & $\begin{array}{c}40 \text { Nephelin, } 34 \text { Mikroklin, } \\
21 \text { Sanidin, } 5 \text { Rutil }\end{array}$ & Feldspäte \\
\hline
\end{tabular}

${ }^{1}$ Ungefähre Glasgehalte nach Bürke, 2006; Kelly, 2008

${ }^{2}$ Glasgehalt der fast gleichwertigen Feldspat-Keramik Vitablocs Mark II

haben (vgl. dazu auch Abb. 89), wobei laut Hersteller der Glasgehalt der Keramik Vitablocs Esthetic Line gegenüber der Standardkeramik Vitablocs Mark II leicht errhöht sein soll. Der berechnete Glasanteil von Vitablocs Esthetic Line nach beiden röntgenograpischen Methoden lag deutlich höher als der nach Herstellerangaben für Vitablocs Mark II (Tab 25). Abweichungen sind hier sehr wahrscheinlich nicht auf die unterschiedliche Röntgenabsorption zurückzuführen, da Glasmatrix und Mineralphasen ähnliche atomare Massen hatten. Das Problem in der Bestimmung des Glasanteils liegt eher in der Art, wie die Keramik fabriziert wurde und welches Korngefüge sie aufweist. Im Herstellungsprozess dieser Keramik wurden natürliche Feldspäte zu Fritten verarbeitet, mit Farbfritten angereichert, zwischengebrannt und zum Schluss im Strangpressverfahren in einen CAD/CAM-Block geformt, wobei dieser in einem letzten Brand bei Temperaturen $>1000^{\circ} \mathrm{C}$ fertiggestellt wurde (Besten et al., 2007). Bei diesen hohen Temperaturen kam es sehr wahrscheinlich zu einer partiellen Schmelze der Feldspatkristalle, da der Liquidus im wasserfreien Albit-Sanidin-System bei $1060^{\circ} \mathrm{C}$ liegt (Barth, 1965) und diese kritische Temperatur während der verschiedenen Brennvorgänge sehr wahrscheinlich erreicht wurde. Zudem wird die Liquidus-Temperatur durch Wasser gesenkt und auch zusätzliche Elemente können den Liquidus dieser Keramik beeinflussen. Durch die Bildung partieller Schmelzen ist eine sehr variable Korngrößenverteilung mit einem fließenden Übergang von großen Kristalliten über immer kleinere Kristallite bis hin zum Glas entstanden. Diese fließende Korngrößenverteilung war auch in den RE-Bildern gut sichtbar (Abschnitt 5.2, Abb. 37). Für die röntgenographische Phasenanalyse mit Bestimmung des Glasgehalts liegt nun das Problem vor, ab wann ein Kristall röntgenographisch noch als Kristall erfasst wird und ab welcher Korngröße nicht mehr. Große Kristallite zeigen eindeutige, scharfe Reflexe im Beugungsbild, während die Reflexe kleinerer Kristalle mit abnehmender Korngröße immer breiter und unschärfer werden. In der Regel geht man daher - für röntgenographische Untersuchungen - von einer optimalen Korngröße von $10 \mu \mathrm{m}$ aus; im vorliegenden Fall jedoch variieren die Korngrößen zwischen $\leq 1 \mu \mathrm{m}$ und $30 \mu \mathrm{m}$. Diese Variation in der Korngröße kann zu deutlichen Unterschieden in der Bestimmung des Glasgehalts nach röntgenographischen oder elektronenmikroskopischen Methoden führen. 


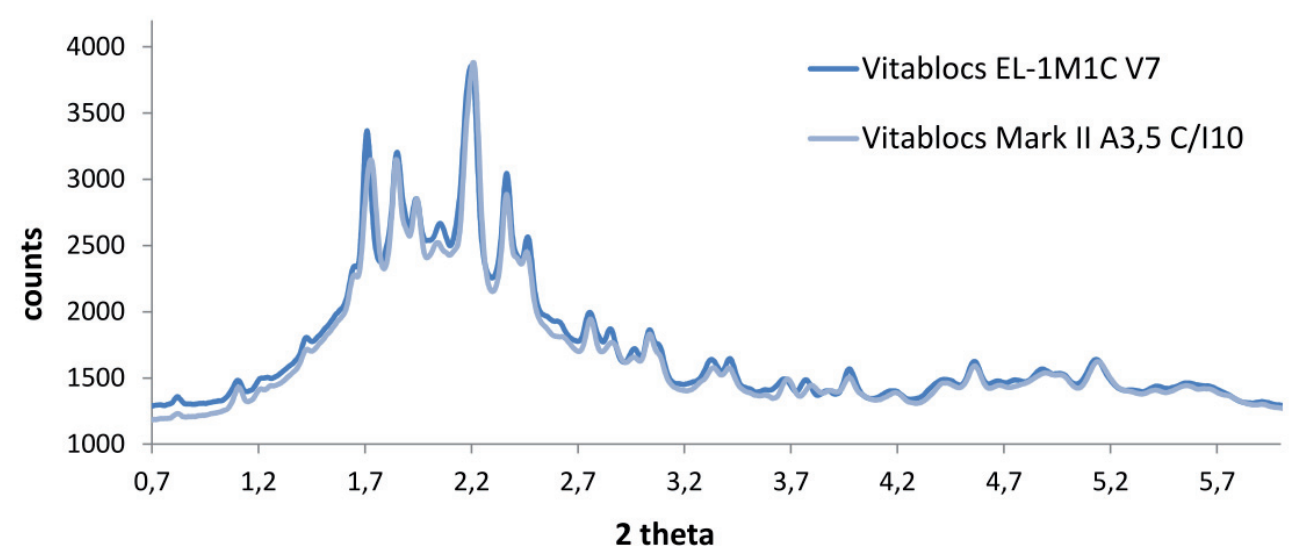

Abb. 89: Röntgendiffraktogramm von Vitablocs Esthetic Line und Vitablocs Mark II. Die Ähnlichkeit der Reflexlagen und -intensitäten sowie der fast gleiche Untergrundverlauf deuten auf eine ähnliche mineralogische Zusammensetzung und Glasgehalt hin.

Für die Dentalkeramik IPS e.max Press liegen keine Herstellerangaben über den Glasgehalt vor, wobei dieser anhand eines wissenschaftlichen Berichtes von Bürke (2006) und nach Kelly (2008) auf 30-40 Vol.\% der fertigen Dentalkeramik eingeschätzt werden kann (Tab. 25). Die hier vorliegenden, röntgenographischen Untersuchungen sind mit diesem Wert nur begrenzt vergleichbar, da die Glasgehalte an einem Keramikrohling und an einem Gusskanal bestimmt wurden, wobei der Gusskanal in der mineralogischen Zusammensetzung der endgesinterten Dentalkeramik sehr nahekommt. Insgesamt wurde im Gusskanal ein höherer Glasgehalt als im Rohling nachgewiesen (Tab. 21). Die Streuung der Ergebnisse ist jedoch je nach angewandter Methode und verwendetem Behältermaterial so groß, dass schwer abzuschätzen ist, welche der Berechnungen (zwischen 25 Gew.\% und 53 Gew.\% für den Gusskanal) der Wahrheit am nächsten kommt. Anscheinend ist im Fall dieser Glaskeramik die röntgenographische Bestimmung des Glasgehaltes aufgrund der vielen, z.T. niedrig symmetrischen Mineralphasen und der ungünstigen Reflexüberlappungen der $\mathrm{Li}_{2} \mathrm{Si}_{2} \mathrm{O}_{5}$-Phase mit denen des Al-Behälters nicht mehr aussagekräftig. 


\subsection{Vergleich der Texturberechnung nach der harmonischen Methode mit der Texturberechnung nach der E-WIMV-Methode}

Am Beispiel des Ti-Wurzelstiftes der Firma Gebr. Brasseler wurde die Orientierungsverteilungsfunktion sowohl nach der harmonischen Methode (aus konventionell gemessenen Polfiguren und aus Synchrotrondaten) als auch nach der E-WIMV-Methode (aus Synchrotrondaten) berechnet. Für die Berechnung nach der harmonischen Methode wurde eine orthorhombische Probensymmetrie angenommen, während für die Berechnung nach der E-WIMV-Methode ohne Symmetrievorgabe gerechnet werden konnte. Allerdings wird auch aus den Polfiguren, die nach der E-WIMVMethode ohne Vorgabe einer Probensymmetrie gerechnet wurden (vgl. Kapitel 5.6.11 Abb. 71), ersichtlich, dass die Annahme einer orthorhombischen Probensymmetrie sinnvoll ist. Ein weiterer Unterschied ist, dass bei der Auswertung der Synchrotrondaten nach der kombinierten RietveldTexturanalyse (E-WIMV-Methode) auch Profilparameter und atomare Auslenkungsparameter verfeinert werden.

Insgesamt sind die Ergebnisse aus den Berechnungen nach der harmonischen Methode mit den Ergebnissen aus der Berechnung nach der E-WIMV-Methode vergleichbar: beim Synchrotrondatensatz werden nach beiden Methoden die gleichen Texturkomponenten mit unterschiedlich hohen Maximalintensitäten berechnet. Während nach der E-WIMV-Methode Intensitätsmaxima von bis zu 18fach in der ODF erreicht werden, werden aus dem gleichen Synchrotrondatensatz nach der harmonischen Methode Werte von 12fach in der ODF erzielt. Beim Vergleich der Texturkomponenten, die aus dem Synchrotrondatensatz berechnet wurden, mit den Texturkomponenten, die aus konventionellen Polfigurmessungen nach der harmonischen Methode berechnet wurden, fällt jedoch auf, dass die unterschiedlichen Texturkomponenten nicht allein durch den anderen Betrachtungswinkel der Probe erklärt werden können (vgl. Kapitel 5.6.11). Möglicherweise können die Unterschiede in diesem Fall darauf zurückzuführen sein, dass bei den konventionellen Polfigurmessungen nur unvollständige Polfiguren gemessen wurden. Die nicht gemessenen Bereiche können bei zu wenigen Polfiguren zu leicht abweichenden Orientierungsverteilungsfunktionen führen. Außerdem war die Kornstatistik bei den konventionellen Polfigurmessungen wesentlich geringer als bei den Synchrotronmessungen, was auch zu Unterschieden in der Texturstärke führen kann.

Neben der besseren Kornstatistik und Zählstatistik ist ein weiterer Vorteil der kombinierten Rietveld-Texturanalyse aus Synchrotrondaten, dass Polfiguren auch aus überlappenden Reflexen gewonnen werden können, während für die Auswertung nach der harmonischen Methode Polfiguren von nicht-überlappenden Reflexen benötigt werden. Dieses ist besonders von Vorteil, wenn die zu untersuchende Phase aufgrund ihrer Kristallstruktur viele überlappende Reflexe aufweist oder es sich um ein Phasengemisch handelt. 


\subsection{Vergleich der Härte- und E-Modulwerte aus dem instrumentierten Eindringverfahren mit anderen Untersuchungen}

Im Fall der Vickershärte erschweren die verschiedenen Messbedingungen, besonders die Verwendung unterschiedlicher Prüflasten und Prüfverfahren, den Vergleich der hier untersuchten Proben mit Literaturwerten. Inwieweit die Härtewerte dennoch vergleichbar sind, wird in den folgenden Abschnitten besprochen. Eine geringere Abhängigkeit von den Messbedingungen ist für den EModul zu erwarten.

\section{Direkte Füllmaterialien: Dentalkomposite, GIZ und Amalgam}

Teilweise sind gute Übereinstimmungen der gemessenen Härtewerte mit Literaturwerten zu erkennen (Tab. 26). Die Abweichungen können entweder durch die unterschiedlichen Messbedingungen erklärt werden oder damit, dass der Kunststoffanteil innerhalb der Komposite unterschiedlich stark ausgehärtet ist. Die Wahl der Prüflast scheint hier den geringeren Einfluss auf die ermittelten Härtewerte zu haben, da alle Werte aus Mikrovickershärteversuchen stammen (hier HV 0,01-0,1) und der „indentation size effect“ (Pharr et al., 2010) vernachlässigbar klein ist. Somit liegt die Vermutung nahe, dass es größere Unterschiede im Grad der Polymeraushärtung gibt. Diese Unterschiede können auf der verwendeten Lichtquelle und der Bestrahlungsdauer beruhen, aber auch darauf, wie lange der Kunststoff an Tageslicht nachgehärtet ist. Im Fall des Glasionomerzementes ist zudem gut erkennbar, dass nicht alle Dentalwerkstoffe homogen sind. Diese Inhomogenität führt bei zu wenigen Messpunkten auch zu Schwankungen in der Vickershärte.

Für den E-Modul der Proben liegen Herstellerangaben nur zu Ceram X und Cimara Zirkon vor. Die Größenordnungen dieser Werte stimmen mit den vorliegenden Ergebnissen überein, wobei laut Herstellerangaben beide Dentalkomposite etwas niedrigere E-Modulwerte aufweisen sollten (Tab. 26). Die Ergebnisse aus anderen Studien, die meistens im instrumentierten Eindringverfahren ermittelt wurden, variieren sehr. Allerdings fehlen oft genaue Angaben zur Berechnung des E-Moduls, z.B. welche Poissonzahl für die Dentalkomposite angenommen wurde. Gute Übereinstimmung lässt sich dagegen für das Amalgam Amalcap regular beobachten.

\section{Prothesenkunststoffe}

Für die Prothesenkunststoffe liegen keine Herstellerinformationen über die Vickershärte und den E-Modul vor. In der weiterführenden Literatur wurden verschiedene Prothesenkunststoffe, darunter Pala Press, und ein Artiplus-Kunststoffzahn untersucht. Der Artiplus-Zahn ähnelt in seiner chemischen Zusammensetzung dem Bioplus-Zahn (interpenetriertes Silikon-Copolymer-Netzwerk ohne Füllpartikel) und kann daher als Vergleich herangezogen werden. Für Artiplus wurde als Härtewert 17,3(0,3) HV 0,1/30 von Campanha et al. (2005) ermittelt, der somit unterhalb des hier gemessenen Härtewerts von 28(1) HV 0,01/20 liegt.

Danesh et al. (2006a,b) haben für Pala Press $E=2,6(1,3) \mathrm{GPa}$ und 27,5(5,7) HV 0,05/30 bestimmt und stimmen im $1 \sigma$-Fehler mit den hier vorliegenden Ergebnissen von 3,4 GPa $(E)$ und 23(4) HV 0,01/20 überein. Die weiteren Prothesenkunststoffe Weitur Press und Aesthetic bestehen aus einer ähnlichen PMMA-Basis und wurden unter gleichen Druck- und Temperaturbedingungen wie Pala Press hergestellt. Daher erscheinen die hier vorliegenden Ergebnisse für $E$ und HV dieser Prothesenkunststoffe, die im $2 \sigma$-Bereich mit denen von Pala Press übereinstimmen, als sinnvoll (Tab. 24). 


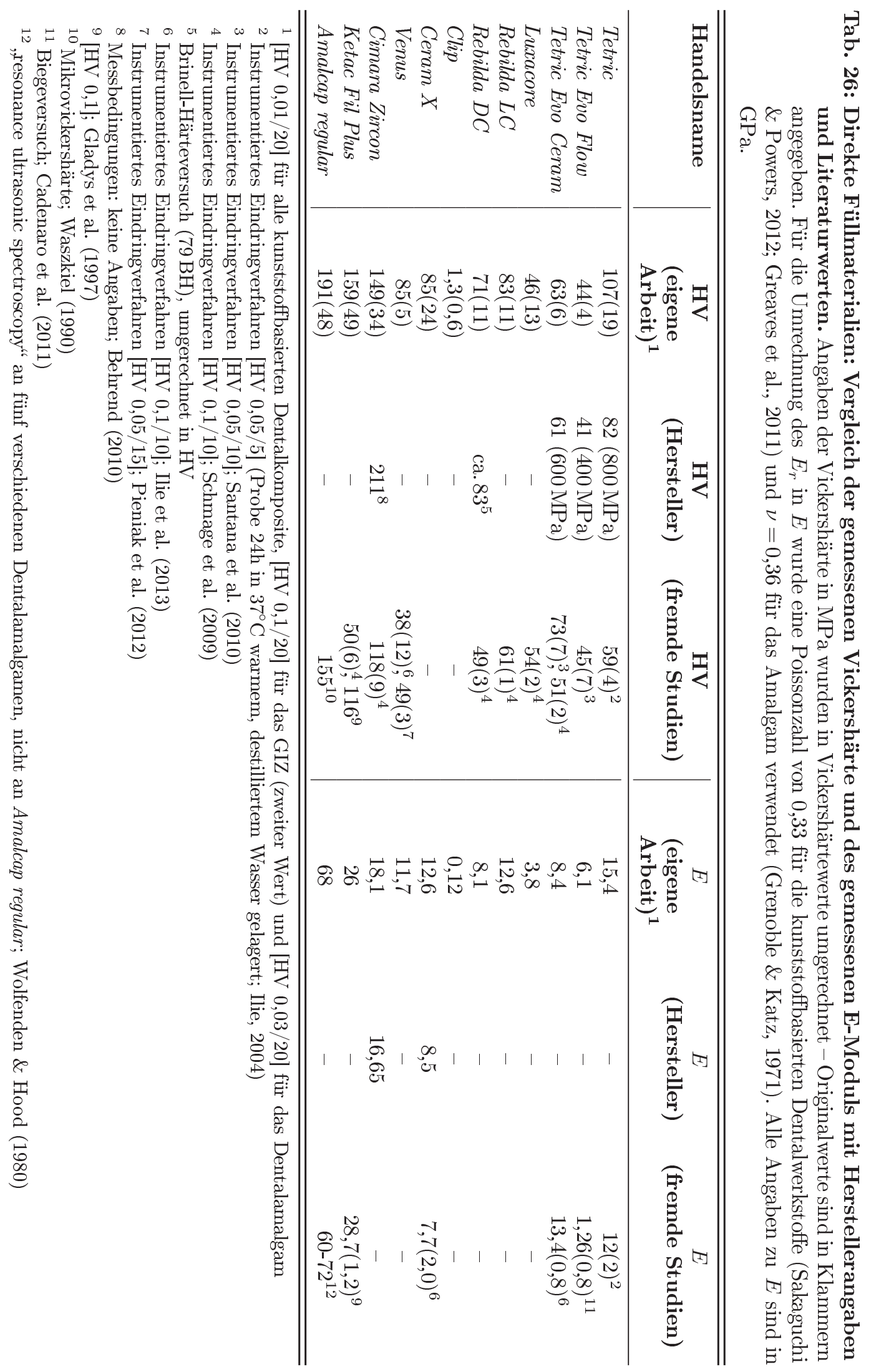




\section{Indirekte Füllmaterialien: Dentalkeramiken}

Für alle Dentalkeramiken sind die Vickershärtewerte nach Herstellerangaben und aus weiteren Studien insgesamt niedriger als die Vickershärtewerte dieser Arbeit (Tab. 27). Diese systematischen Abweichungen sind sehr wahrscheinlich auf unterschiedliche Prüflasten zurückzuführen, da die vorliegenden Untersuchungen im Mikrohärtebereich $(<1,96 \mathrm{~N} \approx 200 \mathrm{~g})$ durchgeführt wurden, während die Untersuchungen aus anderen Studien im Kleinlastbereich $(1,96-49 \mathrm{~N} \approx 0,2-5,0 \mathrm{~kg})$ oder sogar im Makrohärtebereich $(>49 \mathrm{~N} \approx 5 \mathrm{~kg})$ vorgenommen wurden.

Die E-Modulwerte dagegen zeigen eine gute Übereinstimmung mit den Herstellerangaben und weiteren Untersuchungen (Tab. 27). Dabei ist jedoch für die Glasinfiltrationskeramiken Vita InCeram Alumina und Zirconia zu berücksichtigen, dass diese Ergebnisse nur bedingt mit den Analysen von Guazzato et al. (2002) vergleichbar sind, da in dieser Arbeit die Proben nicht im CAD/CAM-Verfahren, sondern im Schlickerverfahren hergestellt wurden.

Tab. 27: Dentalkeramiken: Vergleich der gemessenen Vickershärte und des E-Moduls mit Herstellerangaben und Literaturwerten. Alle Daten beziehen sich auf Keramiken im endgesinterten Zustand [HV 0,03/20]. $E$ wurde aus $E_{r}$ unter der Annahme berechnet, dass die tatsächliche Poissonzahl $\nu$ für Dentalkeramiken zwischen 0,21 und 0,26 liegt (Sakaguchi \& Powers, 2012); für die Vita In-Ceram-Keramiken liegen genauere Werte vor: $\nu_{\text {Alumina }}=0,25$ und $\nu_{\text {Zirconia }}=0,26$ (Guazzato et al., 2002). Alle Angaben zu $E$ sind in GPa.

\begin{tabular}{l|ccc|ccc}
\hline \hline Handelsname & $\begin{array}{c}\text { HV } \\
\text { (eigene } \\
\text { Arbeit) }\end{array}$ & $\begin{array}{c}\text { HV } \\
\text { (Her- } \\
\text { steller) }\end{array}$ & $\begin{array}{c}\text { HV } \\
\text { (fremde } \\
\text { Studien) }\end{array}$ & $\begin{array}{c}E \\
\text { (eigene } \\
\text { Arbeit) }\end{array}$ & $\begin{array}{c}E \\
\text { (Her- } \\
\text { steller) }\end{array}$ & $\begin{array}{c}E \\
\text { (fremde } \\
\text { Studien) }\end{array}$ \\
\hline Cercon base & $827(340)$ & - & - & $193-198$ & 210 & - \\
Vitablocs Mark II, A 3.5 & $661(77)$ & $640(20)^{1}$ & $569(10)^{2}$ & $62-64$ & $63(0,5)$ & - \\
Vitablocs EL & $662(58)$ & - & - & $60-62$ & - & - \\
Vita In-Ceram Alumina & $1374(140)$ & - & $1173(5)^{3}$ & 289 & 280 & $290)^{4}$ \\
Vita In-Ceram Zirconia & $1203(281)$ & - & $1020(2)^{3}$ & 231 & 258 & $242(10)^{3}$ \\
IPS e.max Press & $733(41)$ & $580-600^{5}$ & $423,3(2)^{6}$ & $95-98$ & $90-100$ & - \\
IPS e.max CAD & $695(84)$ & $580-600^{5}$ & $545,7(2)^{6}$ & $95-98$ & $90-100$ & - \\
\hline \hline
\end{tabular}

${ }^{1}$ [HV 0,1/15]; VIDENT (2002)

${ }^{2}$ [HV 10/10]; Charlton et al. (1970)

${ }^{3}$ [HV 0,5/15; HV 1/15]; Proben im Schlickerverfahren hergestellt (Guazzato et al., 2002)

${ }^{4}$ Resonanzfrequenzanalyse (Fischer et al., 2001)

${ }^{5}$ Messbedingungen: k.A. (Behrend, 2010)

${ }^{6}$ [HV 10/20]; Mohsen (2011) 


\section{Dentalmetalle und -legierungen}

Die gemessenen Vickershärtewerte der Dentallegierungen sind im Vergleich zu den Herstellerangaben erhöht. Diese erhöhten Härtewerte sind sehr wahrscheinlich auf unterschiedliche Prüflasten zurückzuführen. Leider wurden nur im Fall der NEM-Legierungen Wiron 99 und Remanium GM $800+$ die verwendeten Prüflasten $(10 \mathrm{~kg})$ angegeben, die damit deutlich über der hier verwendeten Prüflast $(0,03 \mathrm{~kg})$ liegen. Neben den Herstellerangaben lagen keine weiteren Literaturwerte zur Vickershärte der Dentallegierungen vor.

Der E-Modulwert der Ni-Cr-Mo-Legierung Wiron 99 passt gut zu den Herstellerangaben, während bei der Co-Cr-Mo-Legierung Remanium GM 800+ ein niedrigerer Wert ermittelt wurde (Tab. 28). $\mathrm{Zu}$ den Goldlegierungen Degudent $U$ und Degulor $M$ liegen keine Herstellerangaben über deren E-Modulwerte vor. An den Legierungen Degulor $M$ und Remanium GM 800+ wurde $E$ aus Biegeversuchen berechnet (Mahmoud et al., 2005), wobei höhere $E$-Werte als in dem hier angewendeten instrumentierten Eindringverfahren festgestellt wurden.

Für den Wurzelstift aus kommerziell reinem Titan liegen keine Herstellerangaben vor, dafür aber allgemeine Studien zum E-Modul von polykristallinem Titan, deren E-Modulwerte leicht oberhalb des hier ermittelten Wertes sind (Tab. 28).

Tab. 28: Dentalmetalle und -legierungen: Vergleich der gemessenen Vickershärtewerte und der E-Module mit Herstellerangaben und Literaturwerten. Eigene Ergebnisse stammen aus dem instrumentierten Eindringverfahren [HV 0,03/20]. Alle Angaben zu $E$ sind in $\mathrm{GPa}$.

\begin{tabular}{l|ccccc}
\hline \hline & \multicolumn{2}{|c}{ Eigene Arbeit } & \multicolumn{2}{c}{ Herstellerangaben } & $\begin{array}{c}\text { fremde } \\
\text { Studien }\end{array}$ \\
& HV & $E$ & HV & $E$ & $E$ \\
\hline Handelsname & $309(12)$ & 90 & - & - & $\approx 113^{1}, 102-104^{2}$ \\
Degudent $U^{3}$ & $272(26)$ & $76^{4}$ & $240^{5}, 210^{6}$ & - & - \\
Degulor $M^{3}$ & $330(34)$ & $51^{4}$ & $235^{5}$ & - & $90^{7}$ \\
Wiron 99 & $264(25)$ & $211^{8}$ & $180^{9}$ & 205 & - \\
Remanium GM 800+ & $582(89)$ & $164^{10}$ & $370(37)^{9}$ & $230(23)$ & $220^{7}$ \\
\hline \hline
\end{tabular}

${ }^{1}$ Kugelmittelwert für Reintitan (hcp) aus Einkristalldaten (Tromans, 2011)

${ }^{2}$ Werte für $\alpha$-Ti (kommerziell rein: Ti Grad I-IV; McCracken, 1999)

${ }^{3}$ Messungen am Gusskanal vorgenommen

${ }^{4}$ Umgerechnet mit $\nu=0,43$ nach Kumar et al. (2003)

${ }^{5}$ Ausgehärtet

${ }^{6}$ Mit Keramik verblendet

${ }^{7}$ Biegeversuch; Mahmoud et al. (2005)

${ }^{8}$ Umgerechnet mit $\nu=0,28$ nach Käse \& Tesk (1989)

${ }^{9}$ Härteversuch, HV 10

${ }^{10}$ Umgerechnet mit $\nu=0,3$ nach Kumagai et al. (2005) 


\subsection{Vergleich der Ergebnisse aus Texturanalysen mit Ergebnissen aus dem instrumentierten Eindringverfahren und mit Literaturwerten}

Im Folgenden sollen die aus Texturanalysen ermittelten, anisotropen Eigenschaften der Dentalwerkstoffe mit Ergebnissen aus dem instrumentierten Eindringverfahren sowie mit Herstellerangaben und weiteren Literaturwerten verglichen werden. Hierbei muss berücksichtigt werden, dass oft nur die makroskopischen, physikalischen Eigenschaften für die einzelnen Mineralphasen angeben werden können. Diese Mineralphasen stehen aber in Wechselwirkung zueinander und können sich gegenseitig beeinflussen. Trotzdem eignen sich auch bei polykristallinen Materialien die berechneten anisotropen, physikalischen Eigenschaften der einzelnen Phasen um einen Trend in den makroskopischen Eigenschaften des Gesamtwerkstoffs aufzuzeigen.

\section{Dentalkeramiken}

Die aus der Texturanalyse berechneten anisotropen E-Modulwerte der kristallinen Phasen liegen deutlich über den E-Modulwerten, die im Härteversuch an der Gesamtprobe ermittelt wurden, und auch über denen nach Herstellerangaben (Tab. 29). Dieser Unterschied ist sehr wahrscheinlich auf die Nichtberücksichtigung der Glasmatrix zurückzuführen, die bei allen Keramiken einen niedrigeren E-Modul aufweist:

- Vergleichbarer E-Modul für die Glasmatrix von In-Ceram Alumina und Zirconia (25 bzw. 20 Gew.\%; chemische Zusammensetzung s. Abschnitt 5.3 Tab. 9): von 110-130 GPa (LaAl-Si-Gläser; Hwa et al., 2004)

- Vergleichbarer E-Modul für die Glasmatrix der Feldspatkeramik Vitablocs Esthetic Line ( $\geq 70$ Gew.\% der gesamten Keramik): ca. 60 GPa (Na-K-Al-Si-Gläser; Hall, 1930)

Zudem wird bei der Keramik In-Ceram Zirconia ein Teil der $\mathrm{Al}_{2} \mathrm{O}_{3}$-Phase durch $\mathrm{ZrO}_{2}$ ersetzt, das ebenfalls einen niedrigeren E-Modul hat. Dadurch ist der im Gegensatz zur In-Ceram Alumina-Keramik leicht geringere E-Modul zu erklären.

Tab. 29: Dentalkeramiken: Vergleich der E-Modulwerte aus den Texturanalysen und aus dem instrumentierten Eindringverfahren [HV 0,03/20] ermittelten E-Module mit Herstellerangaben.

\begin{tabular}{l|lccc}
\hline \hline & \multicolumn{2}{c}{$E(\mathbf{G P a})$ der kristallinen Phasen } & \multicolumn{2}{c}{$E$ (GPa) der Gesamtprobe } \\
& Hauptphase & Nebenphasen & Härteversuch Hersteller \\
\hline In-Ceram Alumina & Korund: 474 & - & 289 & 280 \\
In-Ceram Zirconia & Korund: 472-475 & $\begin{array}{c}\mathrm{ZrO}_{2} \text {-t: } 262 ; \\
\mathrm{ZrO}_{2} \text {-m: 205 }\end{array}$ & 231 & 258 \\
\hline \multirow{2}{*}{ Vitablocs EL } & Nephelin: 92 & $\begin{array}{l}\text { Mikroklin: 94-99 } \\
\text { Sanidin: 106-113 }\end{array}$ & $60-64$ & 63 \\
\hline \hline
\end{tabular}


Des Weiteren liegen zu den Dentalkeramiken Herstellerangaben über die thermische Ausdehnung vor (Tab. 30). Die aus den Texturanalysen berechneten Werte für die thermische Ausdehnung der kristallinen Phasen liegen meist unter den Werten des Herstellers der entsprechenden Gesamtkeramik. Wie schon beim Vergleich der E-Modulwerte ist dieser Unterschied sehr wahrscheinlich auf das andere physikalische Verhalten der Glasphase zurückzuführen:

- Vergleichbarer Wert für die thermische Ausdehnung (WAK: Wärmeausdehnungskoeffizient) für die Glasmatrix von In-Ceram Alumina und Zirconia: $6,3-8,7 \cdot 10^{-6} / \mathrm{K}\left(25-500^{\circ} \mathrm{C}\right.$; LaAl-Si-Glas mit jedoch 16 Gew. $\% \mathrm{~B}_{2} \mathrm{O}_{3}$ und $10 \mathrm{Gew} . \% \mathrm{TiO}_{2}$; Jang et al., 2004)

- Vergleichbarer WAK für die Glasmatrix von IPS e.max Press und CAD: um $12,8 \cdot 10^{-6} / \mathrm{K}$ (200-400 ${ }^{\circ}$; $\mathrm{Li}_{2} \mathrm{Si}_{2} \mathrm{O}_{5}$-Glaskeramik; Mastelaro \& Zanotto, 1999)

- Vergleichbarer WAK für die Glasmatrix der Feldspatkeramik Vitablocs Esthetic Line: $10-13 \cdot 10^{-6} / \mathrm{K}\left(20-400^{\circ} \mathrm{C}\right.$; Na-K-Al-Si-Gläser; Hall, 1930)

Tab. 30: Dentalkeramiken: Vergleich der aus den Texturanalysen ermittelten thermischen Ausdehnung mit Herstellerangaben. Alle Angaben sind in $10^{-6} / \mathrm{K}$. $\mathrm{Zu}$ den mit * gekennzeichneten Mineralphasen liegen keine Literaturwerte vor.

\begin{tabular}{|c|c|c|c|}
\hline & \multicolumn{2}{|c|}{ WAK der kristallinen Phasen } & \multirow{2}{*}{$\begin{array}{c}\text { WAK der } \\
\text { Gesamtkeramik } \\
\text { Hersteller }\end{array}$} \\
\hline & Hauptphase & $\begin{array}{c}\text { Nebenphasen } \\
(>5 \text { Gew.\%) }\end{array}$ & \\
\hline In-Ceram Alumina & Korund: $5,45^{1}$ & - & $7,2\left(25-500^{\circ} \mathrm{C}\right)$ \\
\hline In-Ceram Zirconia & Korund: $5,42-5,49^{1}$ & $\mathrm{ZrO}_{2}$-t: 5,26 & $7,7\left(25-500^{\circ} \mathrm{C}\right)$ \\
\hline $\begin{array}{l}\text { IPS e.max Press } \\
\text { IPS e.max CAD }\end{array}$ & $\begin{array}{l}\mathrm{Li}_{2} \mathrm{Si}_{2} \mathrm{O}_{5}: 10,14-11,09^{2} \\
\mathrm{Li}_{2} \mathrm{Si}_{2} \mathrm{O}_{5}: 10,7^{2}\end{array}$ & $\begin{array}{l}\mathrm{Li}_{3} \mathrm{PO}_{4}: * \\
\mathrm{Li}_{3} \mathrm{PO}_{4}: *\end{array}$ & $9,75-10,55\left(100-400^{\circ} \mathrm{C}\right)$ \\
\hline Vitablocs EL & Nephelin: $6,97^{3}$ & $\begin{array}{c}\text { Mikroklin: * } \\
\text { Sanidin:7,2-8,1 }\end{array}$ & $9,4\left(25-500^{\circ} \mathrm{C}\right)$ \\
\hline
\end{tabular}

${ }^{1}$ Berechnung mit Einkristalldaten nach Shvyd'ko et al. $(2002)\left(25-240^{\circ} \mathrm{C}\right)$

${ }^{2}$ Berechnung mit Einkristalldaten nach Mastelaro \& Zanotto $(1999)\left(25-450^{\circ} \mathrm{C}\right)$

${ }^{3}$ Berechnung mit Einkristalldaten nach Bonczar \& Barsch $(1975)\left(25-300^{\circ} \mathrm{C}\right)$

${ }^{4}$ Berechnung mit Einkristalldaten nach Mastelaro \& Zanotto $(1999)\left(20-500^{\circ} \mathrm{C}\right)$

Insgesamt ist bei den vorliegenden Glaskeramiken schwer abzuschätzen, wie groß der AnisotropieEffekt der physikalischen Eigenschaften tatsächlich ist und welche Auswirkung er auf die makroskopischen Eigenschaften der gesamten Keramik hat. Sicher dagegen ist, dass zwischen den kristallinen Phasen und der Glasmatrix Spannungen durch die unterschiedlichen physikalischen Eigenschaften auftreten können. Dabei ist es fraglich, ob die so aufgebauten Spannungen im Einzelfall kompensiert werden können oder (auf Dauer) zum Bruch der Keramik führen. 


\section{Titan}

In der Zahnmedizin kommt kommerziell reines Titan (cp-Ti) der Grade I-IV zur Anwendung. cp-Ti wurde auch für den Ti-Wurzelstift der Firma Gebr. Brasseler und für das Titan-Implant OsseoSpeed verwendet, wobei für Osseospeed der genaue Reinheitsgrad von IV bekannt war. Insgesamt zeigen die physikalischen Eigenschaften $E$ und sehr wahrscheinlich auch WAK nur eine geringe Abhängigkeit vom Reinheitsgrad (E: 102-104 GPa; McCracken, 1999). Daher werden beide Werkstoffe, die auch in der Texturanalyse ein sehr ähnliches physikalische Verhalten zeigten, im Folgenden zusammen behandelt.

Tab. 31: Titan für zahnmedizinische Anwendungen: Vergleich der aus den Texturanalysen und aus dem instrumentierten Eindringverfahren [HV 0,03/20] ermittelten E-Modulwerte und der thermischen Ausdehnung mit Literaturwerten. Angaben zum E-Modul in GPa - Angaben zur thermischen Ausdehnung in $10^{-6} / \mathrm{K}$.

\begin{tabular}{l|cl}
\hline \hline Eigenschaftsgröße & Wert & Quelle \\
\hline & $160-167$ & diese Arbeit (Texturanalyse) $)^{1}$ \\
& 90 & diese Arbeit (Härteversuch) \\
& $\approx 113$ & Methode der Kugelmittelwerte aus Einkristalldaten (Tromans, 2011) \\
& $102-104$ & McCracken $(1999)^{3}$ \\
& $106-120$ & Roberts $(1962)^{3}$ \\
& $103-108$ & Rüdinger $(1978)^{3}$ \\
\hline \multirow{3}{*}{ WAK $\left(10^{-6} / \mathrm{K}\right)$} & $7,6-9,3$ & diese Arbeit $\left(28-155^{\circ} \mathrm{C} ;\right.$ Einkristalldaten nach Pawar \& Deshpande, \\
& $9,3-9,5$ & $20-400^{\circ} \mathrm{C}$; Rüdinger (1978) \\
\hline \hline
\end{tabular}

${ }^{1}$ Texturanalyse an Ti-Wurzelstift und Ti-Implantat: Ähnliche Maximal- und Minimalwerte

${ }^{2}$ Messung am Ti-Wurzelstift

${ }^{2}$ Verwendete Methode nicht bekannt

Die E-Modulwerte aus den Texturanalysen liegen mit 160-167 GPa deutlich über denen aus dem Härteversuch (90 GPa) und auch über den Literaturwerten (um $105 \mathrm{GPa}$; Tab. 31). Dieser große Unterschied in den Ergebnissen ist nur zum Teil durch die unterschiedlichen Untersuchungsmethoden zu erklären. Bessere Übereinstimmung hingegen lässt sich für die thermische Ausdehnung beobachten (Tab. 31), wobei sich die Literaturwerte im Bereich der oberen Grenze befinden. Hierbei muss jedoch auch der Temperaturbereich berücksichtigt werden, auf den sich der WAK bezieht: Für den aus der Texturanalyse berechneten WAK liegt ein wesentlich kleiner Temperaturbereich von $28-155^{\circ} \mathrm{C}$ vor als für die Literaturwerte von polykristallinem cp-Ti (20-400; Rüdinger, 1978). 


\subsection{Vergleich der Eigenschaften der untersuchten Zahnmaterialien mit natürlichem Zahn}

Besonders interessant ist der Vergleich der physikalischen Eigenschaften der Zahnersatzmaterialien mit denen von natürlichem Zahn. Dazu sollen die mittleren Härte- und E-Modulwerte der Dentalwerkstoffe mit Literaturwerten für Zahnschmelz, Dentin und Kieferknochen verglichen werden (Schematischer Aufbau eines Zahns s. Abb. 90). Neben dem Vergleich der mittleren, isotropen Werte soll auch auf die (oft nicht) vorhandenen Anisotropien der physikalischen Eigenschaften der untersuchten Zahnmaterialien im Vergleich zum natürlichem Zahn, insbesondere dem Zahnschmelz, eingegangen werden.

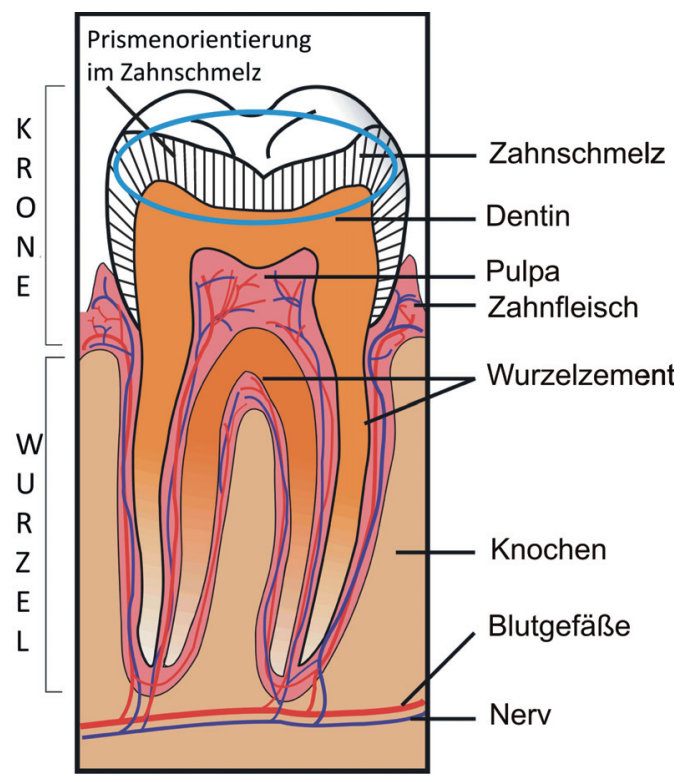

Abb. 90: Schematische Darstellung eines Backenzahns mit eingezeichneter Orientierung der Hydroxylapatit-Prismen im Zahnschmelz (nach Raue, 2013). Zahnschmelz: ca. 97 Gew.\% Hydroxylapatit, 3 Gew.\% organische Bestandteile und Fluide (Young \& Spooner, 1969); Dentin: 70 Gew.\% Hydroxylapatit, 20 Gew.\% organische Bestandteile (Kollagen), 10 Gew.\% Fluide (Nanci, 2008).

Die physikalischen Eigenschaften von Zahnschmelz sind im Allgemeinen nicht leicht zu erfassen, da die Härte und der E-Modul mit zunehmendem Alter ansteigen und auch lokale Variationen aufweisen (Sakaguchi \& Powers, 2012; Kishen et al., 2000). Die lokalen Variationen entstehen durch den unterschiedlich hohen Organik-Anteil des Zahnschmelzes am Zahnhöcker und an der Dentin-Zahnschmelz-Grenze (DEJ: dentino-enamel junction) oder auch durch Kariesbefall. Zudem ändern sich die Eigenschaften von Zahnschmelz und Dentin je nach Lagerungsbedingung der Proben: frisch, trocken eingebettet, in Wasser oder in einer speziellen Lösung gelagert (Habelitz et al., 2002). Zum Beispiel nimmt der E-Modul von trockenem Zahnschmelz um ca. 15\% gegenüber dem von frischem Zahnschmelz zu (Staines et al., 1981). Weiterhin muss berücksichtigt werden, dass sich die physikalischen Eigenschaften wie E-Modul und thermische Ausdehnung anisotrop verhalten. Das bedeutet, dass E-Modul und thermische Ausdehnung richtungsabhängig variieren, also z.B. in Richtung Zahnoberfläche - parallel zur Längsrichtung der HydroxylapatitPrismen - höhere Werte als parallel zur Zahnoberfläche erreichen. 
Auch die Untersuchungen des Dentins zeigen eine Spannbreite in ihren Härtewerten und in ihrem E-Modul. Allerdings liegen bisher noch keine Untersuchungen zum anisotropen Verhalten von physikalischen Eigenschaften des Dentins vor. Eine Übersicht über Vickershärte und EModul von Zahnschmelz, Dentin und Kieferknochen wird in Tab. 32 und 33 gegeben, wobei zum Kieferknochen aufgrund seines porösen Aufbaus keine Vickershärtemessungen vorliegen.

Tab. 32: Vickershärte von trockenem Dentin und Zahnschmelz aus Literaturdaten.

\begin{tabular}{l|cll}
\hline \hline Material & HV & Bemerkungen & Quelle \\
\hline \multirow{3}{*}{ Dentin } & $45,7-54,9$ & Mikrohärte (HV 0,1/0,2/0,3/10/20/30) & Chuenarrom et al. (2009) \\
& 70 & Mikrohärte (HV 0,01/30) & Schmitt et al. (2008) \\
& $17-80$ & Mikrohärte (HV 0,05/15) & Kishen et al. (2000) \\
\hline \multirow{2}{*}{ Zahnschmelz } & 390 & Mikrohärte (HV 0,01/30) & Schmitt et al. (2008) \\
& $316-354$ & Mikrohärte (HV 0,1/0,2/0,3/10/20/30) & Chuenarrom et al. (2009) \\
\hline \hline
\end{tabular}

Tab. 33: E-Modul von trockenem Dentin, Zahnschmelz und Kieferknochen aus Literaturdaten.

\begin{tabular}{|c|c|c|c|}
\hline Material & $E(\mathrm{GPa})$ & Bemerkungen & Quelle \\
\hline \multirow{2}{*}{ Dentin } & $28,6(0,6)$ & Nanoindentation $(400-700 \mu \mathrm{N})$ & Kinney et al. (1999) \\
\hline & $2-20$ & Spannbreite der Literaturwerte bis 1999 & Kinney et al. (1999) \\
\hline \multirow{6}{*}{ Zahnschmelz } & $120-130$ & Zahnhöckerspitze, Nanoindentation & Braly et al. (2007) \\
\hline & $47-120$ & Nanoindentation, von DEJ bis zur & Cuy et al. (2002) \\
\hline & $82-152,6$ & Nanoindentation $(\leq 2 \mathrm{mN} / 5 \mathrm{~s})$ & Raue et al. (2011) \\
\hline & $137-157$ & Texturanalyse mit Einkristalldaten nach & Raue et al. (2011) \\
\hline & & Katz \& Ukraincik (1971) & \\
\hline & 10-169 & $\begin{array}{l}\text { Spannbreite der Literaturwerte bis } 2011 \\
\text { (trocken) }\end{array}$ & Raue et al. (2011) \\
\hline \multirow{2}{*}{ Knochen } & $25(6)$ & $\begin{array}{l}\text { Unterkiefer (zahnlos, } 66 \text { Jahre, Scanning } \\
\text { Acoustic Microscope) }\end{array}$ & Nomura et al. (2007) \\
\hline & $14,9-18,3$ & $\begin{array}{l}\text { Ober- und Unterkiefer (zahnlos, ca. } 83 \text { Jahre, } \\
\text { HV } 0,01 / 10 \text { ) }\end{array}$ & Seong et al. (2009) \\
\hline
\end{tabular}

\section{Vergleich der Vickershärte}

Die Dentalkomposite, die im Stumpfaufbau verwendet werden und somit Kontakt zum Dentin haben, liegen mit ihren Vickershärtewerten (Tab. 34) im Bereich von menschlichem Dentin (Dentin: 70 HV 0,01/30; Schmitt et al., 2008). Die Vickershärtewerte von Dentalkompositen, die für die Kavitätenklassen I-V eingesetzt werden und sowohl Kontakt zum Zahnschmelz als auch zum Dentin haben, sind mit 44-149 HV 0,01/20 im Bereich der Vickershärte von Dentin oder im Vergleich zum Dentin leicht erhöht, erreichen aber nicht die Vickershärte von Zahnschmelz (Zahnschmelz: 390 HV 0,01/30; Schmitt et al., 2008). Im Gegensatz dazu wies das Komposit-Provisorium Clip die niedrigste Vickershärte von 1,3 HV 0,01/20 auf und hat damit eine deutlich geringere Härte als Dentin und Zahnschmelz. Eine ebenfalls geringe Vickershärte besitzen mit $\approx 25$ HV 0,01/20 die Prothesenkunststoffe, wobei die im Vergleich zum Zahn niedrigere Vickershärte in ihrer Funktion als herausnehmbarer Zahnersatz bzw. Schiene durchaus erwünscht ist. Das Glasionomerzement Ketac Fil Plus und das Amalgam Amalcap regular sind 
Tab. 34: Vickershärte und E-Modul nach Materialklassen. Alle Mittelwerte bzw. Minimal- und Maximalwerte wurden aus Messungen nach dem instrumentierten Eindringverfahren der vorliegenden Arbeit übernommen.

\begin{tabular}{l|l|cc}
\hline \hline Material & Anwendung & HV & $E(\mathbf{G P a})$ \\
\hline Prothesenkunststoff & Prothese & $\approx 25$ & $\approx 3,4$ \\
\hline Dentalkomposit & Füllungen der Klasse I-V & $44-149$ & $6,1-18,1$ \\
Dentalkomposit & Stumpfaufbau & $46-71$ & $3,8-12,6$ \\
Dentalkomposit & Provisorium & 1,3 & 0,12 \\
\hline GIZ & Provisorium & $\approx 159$ & 26 \\
\hline Amalgam & Füllungen der Klasse I-V & 191 & 68 \\
\hline Cercon base (Y-ZrO, $)$ & Gerüstkeramik für Kronen, Brücken & 993 & 210 \\
Vita In-Ceram Alumina & Gerüstkeramik für Kronen, Brücken & 1374 & 289 \\
Vita In-Ceram Zirkonia & Gerüstkeramik für Kronen, Brücken & 1203 & 231 \\
Vitablocs (Feldspat) & Gerüstkeramik für Kronen & 662 & $60-64$ \\
Li ${ }_{2}$ Si ${ }_{2} \mathrm{O}_{5}$-Keramik & Gerüstkeramik für Kronen & 714 & $95-98$ \\
\hline Goldlegierung & Kronen & $\approx 301$ & $\approx 64$ \\
Wiron 99 (Ni-Cr-Mo) & Kronen, Brücken & 264 & 164 \\
Remanium GM 800+(Co-Cr-Mo) & Klammermodellguss & 582 & 211 \\
Titan & Wurzelstift, Implantat & 309 & 90 \\
\hline \hline
\end{tabular}

in ihren Vickershärtwerten vergleichbar mit den Dentalkompositen, die für die Kavitätenklassen I-V verwendet werden, und liegen mit ihrer Vickershärte von 159 bzw. 191 HV 0,01/20 zwischen der von Dentin und Zahnschmelz. Gold- und NEM-Legierungen haben eine Vickershärte vergleichbar mit Zahnschmelz, wobei die Co-Cr-Mo-Legierung Remanium GM 800+ mit $582 \mathrm{HV}$ 0,01/20 einen wesentlich höheren Wert erreicht. Eine im Vergleich zu Dentin und Zahnschmelz deutlich erhöhte Vickershärte zeigen die Dentalkeramiken, deren Werte bei mehr als $650 \mathrm{HV}$ $0,01 / 20$ liegen.

\section{Vergleich des E-Moduls}

Das Komposit-Provisorium Clip und die Prothesenkunststoffe haben mit 0,12 GPa und $\approx 3,4 \mathrm{GPa}$ die niedrigsten E-Module (Tab. 34). Darauf folgen die Dentalkomposite für den Stumpfaufbau und für verschiedene Kavitätenklassen $(3,8-18,1 \mathrm{GPa})$. Die E-Modulwerte der Dentalkomposite liegen insgesamt innerhalb der Spannweite, die für Dentin festgestellt wurde (Dentin: 2-28,6 GPa; Kinney et al., 1999), und somit deutlich unter vergleichbaren Werten für Zahnschmelz (47-157 GPa; Raue et al., 2011; Cuy et al., 2002). Die hier angegeben EModulwerte von 47-157 GPa sind nur als Richtwerte für die Variation des E-Moduls innerhalb des Zahnschmelzes zu sehen. Tatsächlich liegt in der Literatur eine noch größere Spannbreite an E-Modulwerten vor, die in Abhängigkeit von der Untersuchungsmethode, aber auch zwischen Studien mit gleicher Untersuchungsmethode variieren (Abb. 91, Raue et al., 2011).

Auch Ketac Fil Plus als Vertreter der Glasionomerzemente hat mit $26 \mathrm{GPa}$ einen E-Modul vergleichbar mit dem E-Modul von Dentin. E-Modulwerte um 60 GPa dagegen erreichen Amalgam, Goldlegierungen und die Feldspatkeramiken der Vitablocs-Serie. Damit liegen sie im unteren Bereich, der für Zahnschmelz gemessen wurde. Etwas höhere Werte von $\approx 90 \mathrm{GPa}$ erreichen die $\mathrm{Li}_{2} \mathrm{Si}_{2} \mathrm{O}_{5}$-Keramiken und auch Titan, das damit einen höheren E-Modul als der umgebende 
Kieferknochen hat (15-25 GPa Nomura et al., 2007; Seong et al., 2009). Die E-Modulwerte der NEM-Legierungen liegen über denen von Zahnschmelz, ebenso die der Glasinfiltrationskeramiken der Vita In-Ceram-Serie.

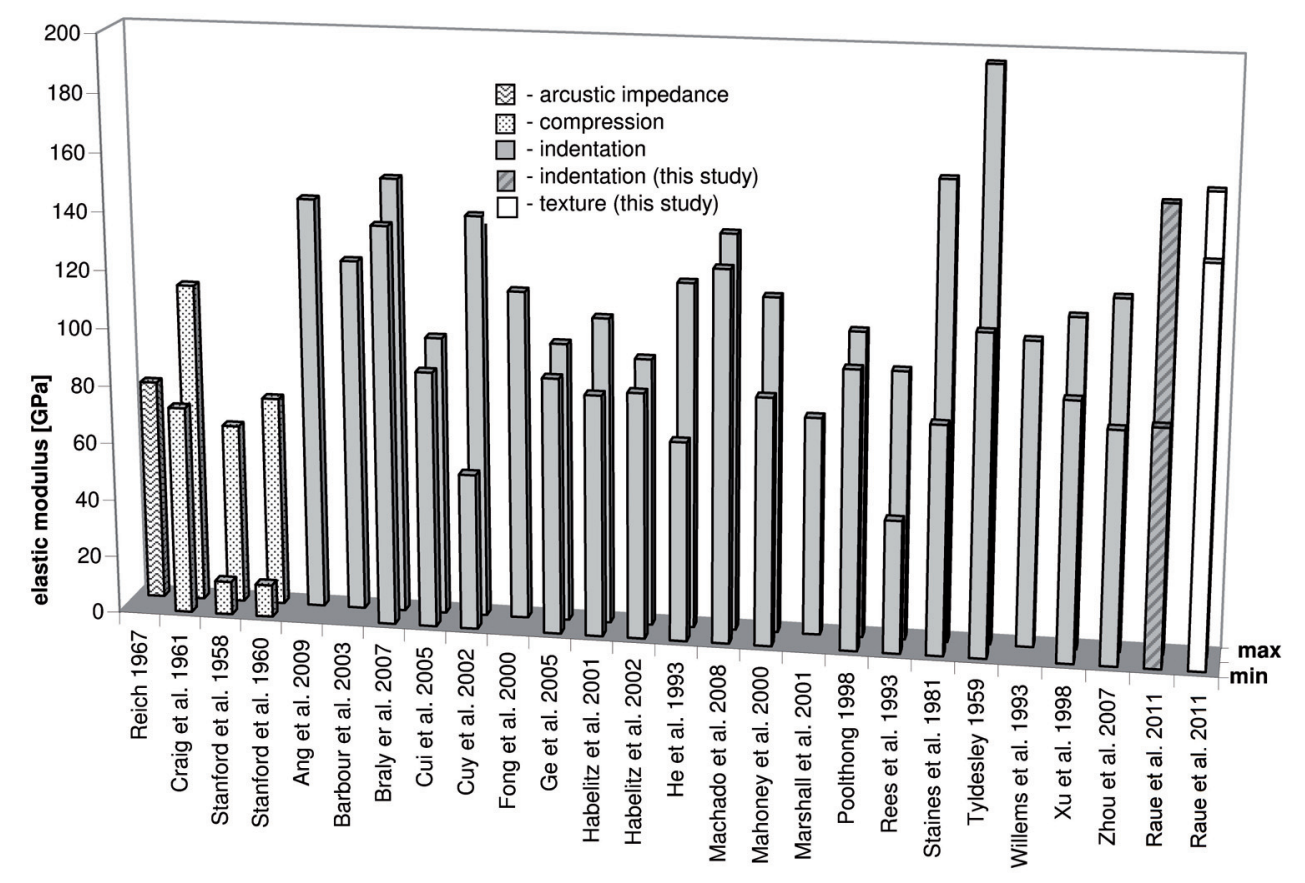

Abb. 91: Zusammenstellung der Literaturdaten für den E-Modulwert von Zahnschmelz. Zur Bestimmung des E-Moduls wurden die akustische Impedanz-Methode, Kompressionsversuche, Nanoindentermessungen und Texturanalyse verwendet. Abbildung nach Raue et al. (2011).

\section{Vergleich der thermischen Ausdehnung}

Für den Vergleich der thermischen Ausdehnung liegen von Xu et al. (1989) isotrope Werte für Dentin $\left(11 \cdot 10^{-6} / \mathrm{K} ; 10-80^{\circ} \mathrm{C}\right)$ und Zahnschmelz $\left(17 \cdot 10^{-6} / \mathrm{K} ; 10-80^{\circ} \mathrm{C}\right)$ vor. Besonders die Dentalkomposite liegen mit $35-75 \cdot 10^{-6} / \mathrm{K}$ deutlich über den Werten des natürlichen Zahns (Abb. 92; 3M ESPE AG, 2013). Einen leicht erhöhten WAK zeigen auch die Dentalamalgame mit 25-35·10 $0^{-6} / \mathrm{K}$ (3M ESPE AG, 2013). Im Bereich von Zahnschmelz und Dentin liegen dagegen das Glasionomerzement Ketac Fil Plus und Goldlegierungen. Unterhalb der thermischen Ausdehnung von menschlichem Zahn sind die meisten Dentalkeramiken sowie Titan, wobei Titan als Wurzelstift oder Implantat auch Kontakt zum Kieferknochen hat, der mit $63 \cdot 10^{-6} / \mathrm{K}$ eine deutlich höhere thermische Ausdehnung besitzt (25-55 $\mathrm{C}$; Huang et al., 2010).

\section{Wie wirkt sich eine Anisotropie der physikalischen Eigenschaften aus?}

Bis hierhin wurde für alle physikalischen Eigenschaften ein isotropes Verhalten angenommen. Mindestens für den Zahnschmelz ist aber bekannt, dass die physikalischen Eigenschaften wie E-Modul und thermische Ausdehnung richtungsabhängig variieren (Raue et al., 2011; Raue \& Klein, 2011). Der E-Modul von Zahnschmelz wurde von Raue et al. (2011) mittels Texturanalysen anisotrop berechnet, wobei Variationen zwischen $137 \mathrm{GPa}$ und $157 \mathrm{GPa}$ festgestellt wurden (Tab. 33). Ebenso variiert die thermische Ausdehnung, für die Werte zwischen $21,25 \cdot 10^{-6} / \mathrm{K}$ und $22,4 \cdot 10^{-6} / \mathrm{K}$ in unterschiedlichen Richtungen ermittelt wurden $\left(22-200^{\circ} \mathrm{C}\right.$; Raue \& Klein, 2011; mit Einkristallwerten von $a_{11}=a_{22}=17,3 \cdot 10^{-6} / \mathrm{K}$ und $a_{33}=24,4 \cdot 10^{-6} / \mathrm{K}$ brechnet, Washburn, 1930). Hierbei fällt auf, dass sowohl der Minimal- als auch der Maximalwert der 


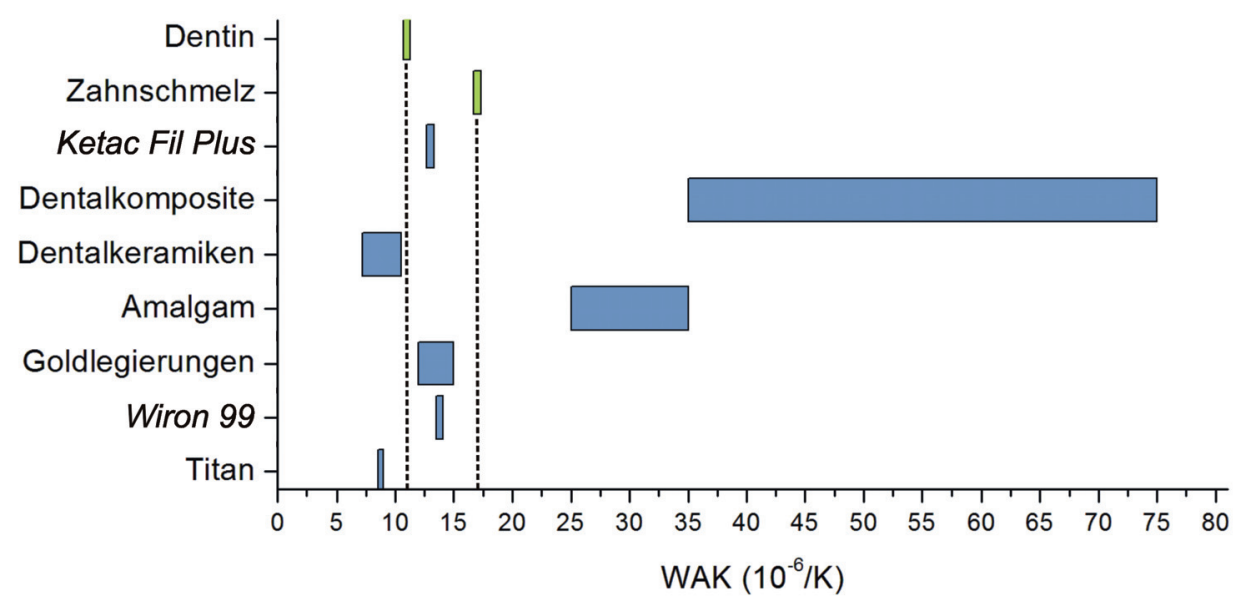

Abb. 92: Vergleich des thermischen Ausdehnungskoeffizienten von Zahnersatzmaterialien mit Dentin und Zahnschmelz. Werte nach Xu et al. (1989) für Dentin und Zahnschmelz, nach 3M ESPE AG (2013) für Ketac Fil Plus, Dentalkomposite und Amalgam, nach Stollwerk (2009) für Goldlegierungen und nach Low et al. (2001) für Titan. Werte für die Dentalkeramiken und die Ni-Cr-Mo-Legierung Wiron 99 wurden den Herstellerangaben entnommen.

anisotropen thermischen Ausdehnung über dem isotropen Wert von $17 \cdot 10^{-6} / \mathrm{K}$ von Xu et al. (1989) liegen. Wahrscheinlich ist dieser Unterschied darauf zurückzuführen, dass bei Raue \& Klein (2011) nur die kristalline Hydroxylapatit-Phase in der Berechnung berücksichtigt wurde, aus der der Zahnschmelz zu 97 Gew.\% besteht, während bei Xu et al. (1989) der Zahnschmelz aus frisch extrahierten Zähnen untersucht wurde.

Insgesamt fällt auf, dass die Abweichungen der (an-)isotropen physikalischen Eigenschaften einzelner Materialklassen vom Dentin und Zahnschmelz größer sind als die richtungsabhängigen Variationen in den physikalischen Eigenschaften von Zahnschmelz, die durch seinen kristallinen Aufbau entstehen. Daher ist zunächst eine bessere Anpassung dieser Eigenschaften an die isotropen Werte von Dentin und Zahnschmelz wünschenswert. Zusätzlich könnte dann im nächsten Schritt die Anisotropie des Zahnschmelzes berücksichtigt werden, indem Zahnersatzwerkstoffe so hergestellt werden, dass sie eine ähnliche Anisotropie der physikalischen Eigenschaften wie der natürliche Zahnschmelz aufweisen. 


\section{Zusammenfassung und Fazit}

Physikalische Eigenschaften wie Härte, E-Modul und Ausdehnungskoeffizient werden bei Dentalwerkstoffen meist als richtungsunabhängig (isotrop) angenommen, obwohl diese Werkstoffe aufgrund ihrer chemischen und kristallographischen Zusammensetzung sowie der Kristallitanordnung richtungsabhängige (anisotrope) Eigenschaften haben können. Da auch der menschliche Zahnschmelz, der zu 97 Gew.\% aus Hydroxylapatit besteht (Young \& Spooner, 1969), eine Anisotropie in seinen physikalischen Eigenschaften aufweist (Raue \& Klein, 2011), müsste der optimale Zahnersatz eine ähnliche Anisotropie wie der zu ersetzende Zahn haben. Auf dem Weg zum optimalen Zahnersatz wurden zunächst bestehende Dentalwerkstoffe mittels Beugungsexperimenten auf ihre mineralogische Zusammensetzung und auf ihre anisotropen physikalischen Eigenschaften wie E-Modul und thermische Ausdehnung untersucht und mit den Eigenschaften von natürlichem Zahn verglichen.

Die Dentalkomposite, ein Glasionomerzement und die Prothesenkunststoffe bestehen aus fast nur amorphen Bestandteilen - i.d.R. Glasfüllpartikel und Kunststoffmatrix - und zeigen insgesamt ein isotropes physikalisches Verhalten: Vickershärtewerte und E-Modul entsprechen in etwa denen von Dentin, während ihre thermische Ausdehnung mit $35-75 \cdot 10^{-6} / \mathrm{K}$ gegenüber Dentin und Zahnschmelz $\left(11 \cdot 10^{-6} / \mathrm{K}\right.$ und $17 \cdot 10^{-6} / \mathrm{K}$; Xu et al. (1989)) wesentlich erhöht ist.

Das $\gamma_{2}$-freie Amalgam bestand aus den kristallinen Phasen $\mathrm{Ag}_{2} \mathrm{Hg}_{3}, \mathrm{Ag}_{3} \mathrm{Sn}$ und $\mathrm{Cu}_{6} \mathrm{Sn}_{5}$ und wies ein isotropes physikalisches Verhalten auf. Seine Vickershärte und sein E-Modul liegen mit 191 HV 0,03/20 bzw. 68 GPa zwischen den Werten für Dentin und Zahnschmelz (70 HV 0,01/30 und 2-29 GPa für Dentin sowie 370 HV 0,01/30 und 10-169 GPa für Zahnschmelz; Schmitt et al., 2008; Kinney et al., 1999; Raue et al., 2011). Seine thermische Ausdehnung ist mit $25,5 \cdot 10^{-6} / \mathrm{K}$ (Kandil et al., 1989) gegenüber natürlichem Zahn leicht erhöht.

Die meisten Dentalkeramiken sind Glaskeramiken und weisen ein z.T. komplexes Mineralgefüge auf: Bei den Keramiken Cercon base und Vita In-Ceram Alumina wurde eine regellose Anordnung der Kristallite beobachtet. Eine leichte Vorzugsorientierung zeigen hingegen Vita In-Ceram Zirkonia und Vitablocs Esthetic Line mit 4,0fach bzw. 3,5fach regellos in der ODF. Nur die $\mathrm{Li}_{2} \mathrm{Si}_{2} \mathrm{O}_{5}$-Keramik IPS e.max Press wies eine starke Vorzugsorientierung auf (11fach regellos in der ODF). Diese Kristallvorzugsorientierungen wirken sich in nur leicht ausgeprägten Anisotropien der makroskopischen physikalischen Eigenschaften aus. Die Dentalkeramiken haben mit $\geq 650 \mathrm{HV}$ 0,01/20 insgesamt deutlich höhere Härtewerte als natürlicher Zahn und liegen mit Ausnahme der Hochleistungskeramiken Cercon base und Vita In-Ceram Alumina/Zirkonia mit ihrem E-Modul von 60-98 GPa zwischen dem von Dentin und Zahnschmelz. Ihre thermische Ausdehnung ist mit $7,2-10,5 \cdot 10^{-6} / \mathrm{K}$ etwas unterhalb der für Dentin.

Goldlegierungen und NEM-Legierungen sind relativ grobkörnig und bilden Aussscheidungsphasen, die zur Härtung der Legierungen beitragen. Die Legierungen für Kronen haben Vickershärtewerte und E-Modulwerte im Bereich von Zahnschmelz, während die Co-Cr-Mo-Legierung Remanium GM 800+, die als Klammermodellguss eingesetzt wird, deutlich härter und elastischer ist (582 HV 0,03/20; $E=211 \mathrm{GPa}$ ). Die thermische Ausdehnung der untersuchten Dentallegierungen liegen zwischen der von Dentin und Zahnschmelz.

Der Titanwurzelstift und auch das Titanimplantat Osseospeed zeigen eine deutlich ausgeprägte Textur (18fach und 19fach regellos in den jeweiligen ODFs), die zu einem leicht anisotropen Verhalten in den physikalischen Eigenschaften wie E-Modul (159-167 GPa) und thermische Ausdehnung $\left(7,62-9,29 \cdot 10^{-6} / \mathrm{K}\right)$ führen. 
Insgesamt sind die Abweichungen in den makroskopischen, physikalischen Eigenschaften der untersuchten Dentalwerkstoffe im Vergleich zu natürlichem Zahn nach beiden Untersuchungsmethoden - instrumentiertes Eindringverfahren und Texturanalyse - größer als die Variationen, die durch ihren kristallinen Aufbau bedingt sind. Daher sollten neue Dentalwerkstoffe zunächst noch besser in ihren isotropen, physikalischen Eigenschaften dem menschlichen Zahn angepasst werden, bevor in einem nächsten Schritt die anisotropen Eigenschaften des Zahnschmelzes imitiert werden. Der optimale Zahnersatzwerkstoff, der am Besten in den physikalischen Eigenschaften dem natürlichen Zahn entspricht, wäre aus dem gleichen Material wie der natürliche Zahn selbst, also aus Hydroxylapatit. Der Apatit sollte auch eine ähnliche Mikrostruktur wie der natürliche Zahnschmelz aufweisen, d.h. er sollte ebenso feinkörnig sein und die Kristallite im Idealfall epitaktisch auf dem Zahnschmelz bzw. Dentin aufgewachsen sein. Dazu liegen erste Versuche vor, wobei anstelle von Hydroxylapatit-Kristalliten säurebeständigere Fluorapatit-Kristallite auf Dentin gezüchtet wurden (Busch, 2008). Dabei gelang es aber bis jetzt nicht die Kristallite so aufwachsen zu lassen, dass die Kristallitorientierung der aufgewachsenen Fluorapatit-Phase mit den Kristallorientierungen des Hydroxylapatits im Dentin oder Zahnschmelz übereinstimmt. Mit der richtigen Kristallitorientierung der Fluorapatitkristallite wäre dieses jedoch der optimale Zahnersatz, der in den physikalischen Eigenschaften und in der Ästhetik dem natürlichen Zahn sehr nahe kommt und durch den Fluorgehalt gegenüber dem Hydroxylapatit säurebeständiger ist. 


\section{Literatur}

3M ESPE AG (2013). Glass ionomers. Informationsbroschüre. Interne Daten der 3M ESPE AG.

Abriel, W. \& Nesper, R. (1993). Bestimmung der Kristallstruktur von $\mathrm{CaSO}_{4}\left(\mathrm{H}_{2} \mathrm{O}\right)_{0,5}$ mit Röntgenbeugungsmethoden und mit Potentialprofil-Rechnungen. Z. Krist., 205, 99-113.

Al-Wahadni, A. (1999). The roots of dental porcelain: a brief historical perspective. Dent. Mater., $6,43-44$.

Albakry, M., Guazzato, M. \& Swain, M. (2003). Biaxial flexural strength, elastic moduli and x-ray diffraction characterization of three pressable all-ceramic materials. J. Prosth. Dent., 89, $374-380$.

Albakry, M., Guazzato, M. \& Swain, M. (2004). Influence of hot pressing on the microstructure and fracture toughness of two pressable dental glass-ceramics. J. Biomed. Mater. Res. B Appl. Biomat., 71B, 99-107.

Albertsson, J., Abrahams, S. C. \& Kvick, A. (1989). Atomic displacement, anharmonic thermal vibration, expansivity and pyroelectric coefficient thermal dependences in $\mathrm{ZnO}$. Acta Cryst. B, $45,34-40$.

Anderson, J. L., Peterson, R. C. \& Swainson, I. P. (2005). Combined neutron powder and x-ray single-crystal diffraction refinement of the atomic structure and hydrogen bonding of goslarite $\left(\mathrm{ZnSO}_{4} \cdot 7 \mathrm{H}_{2} \mathrm{O}\right)$. Mineral. Mag., 69, 259-271.

Angel, R. J., Carpenter, M. A. \& Finger, L. W. (1990). Structural variation associated with compositional variation and order-disorder behavior in anorthite-rich feldspars. Am. Mineral., $75,150-162$.

Arthur, R. (1857). A treatise on the use of adhesive gold foil. Philadelphia: Jones.

Baird, H. W. \& Muller, F. A. (1969). Refinement of the crystal structure of gamma phase silver amalgam. J. Biomed. Mater. Res., 3, 375-382.

Barth, T. F. W. (1965). On the constitution of the alkali feldspars. Tschermaks mineral. petrogr. Mitt., 10, 14-33.

Bear, I. J., Grey, I. E., Madsen, I. C., Newnham, I. E. \& Rogers, L. J. (1986). Structures of the basic zinc sulfates $\left(\mathrm{Zn}(\mathrm{OH})_{2}\right)_{3} \cdot\left(\mathrm{ZnSO}_{4}\right)\left(\mathrm{H}_{2} \mathrm{O}\right)_{m} \mathrm{~m}=3$ and 5. Acta Cryst. B, 42, 32-39.

Behrend, D. (2010). Bericht an VOCO 1-2. Technical report, Universität Rostock, Lehrstuhl für Medizintechnik. 
Besten, O., Bülling, I., Fricke, T. \& Wolff, S. (2007). Wie werden eigentlich CAD/CAM-Blöcke hergestellt? Dent. Dig. News, 1, 50-56.

Black, G. V. (1917). A work on operative dentistry; the technical procedures in filling teeth. Chicago: Medico-dental Publishing Co.

Bochvar, N., Liberov, Y. \& Perrot, P. (2006). The Landolt-Börnstein Database: Thermodynamic Properties - Ternary Alloy Systems: Phase Diagrams, Crystallographic and Thermodynamic Data critically evaluated by MSIT ${ }^{\circledR}$ - Noble Metal Systems. Selected Systems from Ag-Al-Zn to Rh-Ru-Sc. Gold-Copper - Platinum, volume 11B. Springer-Verlag.

Boeyens, J. C. A. \& Ichharam, V. V. H. (2002). Redetermination of the crystal structure of calcium sulphate dihydrate, $\mathrm{CaSO}_{4} \cdot 2 \mathrm{H}_{2} \mathrm{O}$. Z. Krist., 217, 9-10.

Bonczar, L. J. \& Barsch, G. R. (1975). Elastic and thermoelastic constants of nepheline. J. Appl. Phys., 46, 4339-4340.

Bowen, R. L. (1963). Properties of a silica-reinforced polymer for dental restorations. J. Am. Dent. Assoc., 66, 57-64.

Bowen, R. L. (1965). Method of preparing a monomer having phenoxy and methacrylate groups linked by hydroxy glycerol groups. US Patent 3179623.

Bragg, W. L. (1913). Line profiles of neutron powder-diffraction peaks for structure refinement. Proc. R. Soc. London Ser. A, 89, 248-277.

Braly, A., Darnell, L. A., Manna, A. B., Teaford, M. F. \& Weihs, T. P. (2007). The effect of prism orientation on the indentation testing of human molar enamel. Arch. Oral Biol., 52, $856-860$.

Brecker, C. S. (1956). Porcelain baked to gold - a new medium in prosthodontics. J. Prosth. Dent., 6, 85-91.

Bürke, H. (2006). IPS e.max Press and IPS e.max CAD, two state-of-the-art glass ceramics. Report, Research and Development Ivoclar Vivadent, 17, 6-16.

Bukvetskii, B. V. \& Garashina, L. S. (1977). Crystal and chemical study of rhombic trifluorides of samarium, holmium and ytterbium. Koordinatsionnaya Khimiya (USSR), 3, 1024-1029.

Bunge, H. J., Hrsg. (1969). Mathematische Methoden der Texturanalyse. Berlin: AkademieVerlag.

Bunge, H.-J. (1993). Texture analysis in materials science. Göttingen: Cuvillier Verlag.

Busch, S. (2008). Verjüngungskur für die Zähne. DZW Spezial, 5/08, 1-3. 
Cadenaro, M., Codan, B., Navarra, C. O., Marchesi, G., Turco, G., Di Lenarda, R. \& Breschi, L. (2011). Contraction stress, elastic modulus, and degree of conversion of three flowable composites. Eur. J. Oral. Sci., 119, 241-245.

Campanha, N. H., Pavarina, A. C., Vergani, C. E. \& Machado, A. L. (2005). Effect of microwave sterilization and water storage on the Vickers hardness of acrylic resin denture teeth. $J$. Prosthet. Dent., 93, 483-487.

Chan, Y. F., Grimsditch, M., Li, Z., Nevitt, M. V., Robertson, W. M. \& Zouboulis, E. S. (1991). Temperature dependence of the elastic moduli of monoclinic zirconia. J. Am. Ceram. Soc., $74,1742-1744$.

Charlton, J. S., Cordey-Hayes, M. \& Harris, I. R. (1970). A study of the ${ }^{119}$ Sn Moessbauer isomer shifts in some platinum-tin and gold-tin alloys. J. Less-Common Met., 20, 105-112.

Chateigner, D. (2010). Combined analysis: structure-texture-microstructure-phase-stressesreflectivity determination by $x$-ray and neutron scattering. Caen: John Wiley \& Sons.

Chen, K., Yu, T., Zhang, Y. \& Peng, Z. (1982). Tetraauricupride, Cu Au, discovered in China. Acta Geol. Sin. (Engl.), 1982, 111-116.

Chuenarrom, C., Benjakul, P. \& Daosodsai, P. (2009). Effect of indentation load and time on Knoop and Vickers microhardness tests for enamel and dentin. Mater. Res., 12, 473-476.

Collares, F. M., Ogliari, F. A., Lima, G. S., Fontanella, V. R. C., Piva, E. \& Samuel, S. M. W. (2010). Ytterbium trifluoride as a radiopaque agent for dental cements. Int. Endod. J., 43, $792-797$.

Cuy, J. 1., Manna, A. B., Livi, K. J., Teaford, M. F. \& Weihs, T. P. (2002). Nanoindentation mapping of the mechanical properties of human molar tooth enamel. Arch. Oral Biol., 47, 281-291.

Dahlem-Klein, E., Klein, H. \& Park, N. J. (1999). Program system: ODF - Analysis. Cuvillier Verlag.

Danesh, G., Lippold, C., Ziebura, T., Reinhardt, K.-J., Schäfer, E. \& Ehmer, U. (2006a). Invitro investigation on suitability of light-cured resins for interocclusal splints - Part II: Surface hardness. J. Orofac. Orthop., 2, 138-147.

Danesh, G., Lippold, C., Ziebura, T., Reinhardt, K.-J., Schäfer, E. \& Ehmer, U. (2006b). In-vitro investigations on suitability of light-cured resins for interocclusal splints - Part I: Mechanical properties. J. Orofac. Orthop., 2, 127-137.

DeguDent (2013). Cercon Smart Ceramics - Produktbeschreibung und Gebrauchsanweisung. Online: http://www.degudent.de/Kommunikation_und_Service/Download/Cercon/Cercon_ brain_heat_clean/Cercon_Werkstofftabelle_dt.pdf 
Denry, I. L., Baranta, G., Holloway, J. A. \& Gupta, P. K. (2002). Effect of processing variables on texture development in a mica-based glass-ceramic. J. Biomed. Mater. Res. B Appl. Biomat., 64B, 70-77.

Dentalkompakt (2013). Produktinformation: OsseoSpeed (4.5, 5.0). http://www.dentalkompaktonline.de/.

Dollase, W. A. (1986). Correction of intensities for preferred orientation in powder diffractometry: Application of the March Model. J. Appl. Cryst., 19, 267-272.

Donaldson, J. A. (1980a). The use of gold in dentistry - an historical overview. Part I. Gold Bulletin, 13, 117-124.

Donaldson, J. A. (1980b). The use of gold in dentistry - an historical overview. Part II. Gold Bulletin, 13, 160-165.

Dukic, W., Delija, B., Lešic, S., Dubravica, I. \& Derossi, D. (2012). Radiopacity of flowable composite by a digital technique. Oper. Dent., 38-3.

Durussel, P. \& Feschotte, P. (1996). A revision of the binary system Ag-Pt. J. Alloys Compounds, 239, 226-230.

Eichner, K. \& Kappert, H. F. (1996). Zahnärztliche Werkstoffe und ihre Verarbeitung. Hüthig, 6. Auflage.

Every, A. G. \& McCurdy, A. K. (1992). Landolt-Börnstein: Numerical data an functional relationships in science and technology (subvolume a, second and higher order elastic constants). Springer. Editor: Nelson, D. F.

Fairhurst, C. W. \& Cohen, J. B. (1972). The crystal structures of two compounds found in dental amalgam: $\mathrm{Ag}_{2} \mathrm{Hg}_{3}$ and $\mathrm{Ag}_{3} \mathrm{Sn}$. Acta Cryst. B, 28, 371-378.

Fauchard, P. (1728). Le Chirurgien Dentiste. Paris: Jean Mariette.

Ferguson, R. B., Ball, N. A. \& Cerny, P. (1991). Structure refinement of an adularian end-member high sanidine from the Buck Claim pegmatite, Bernic Lake, Manitoba. Can. Mineral., 29, 543552 .

Fischer, H., Dautzenberg, G. \& Marx, R. (2001). Nondestructive estimation of the strength of dental ceramic materials. Dent. Mater., 17, 289-295.

Fisher, E. S. \& Manghani, M. H. (1971). Effects of changes in volume and $c / a$ ratio on the pressure derivatives of the elastic moduli of h.c.p. Ti and Zr. J. Phys. Chem. Solids, 32, $657-667$. 
Gangulee, A., Das, G. C. \& Bever, M. B. (1973). An x-ray diffraction and calometric investigation of the compound $\mathrm{Cu}_{6} \mathrm{Sn}_{5}$. Met. Trans., 4, 2063-2066.

Ghosh, G. (2004). Elastic properties, hardness, and indentation fracture toughness of intermetallics relevant to electronic packaging. J. Mater. Res., 19, 1439-1454.

Gladys, S., Van Meerbeek, B., Braem, M., Lambrechts, P. \& Vanherle, G. (1997). Comparative physico-mechanical characterization of new hybrid restorative materials with conventional glass-ionomer and resin composite restorative materials. J. Dent. Res., 76, 883-894.

Gomez-Acebo, T. (1998). Thermodynamic assessment of the Ag-Zn system. Calphad, 22, 203220.

Graf, K. (2013). Zahnärztliche Werkstoffe unter umweltmedizinischen Aspekten. Online: http: //www.integrative-zahnheilkunde.de/kunststoffeundcomposite.html.

Greaves, G. N., Greer, A. L., Lakes, R. S. \& Rouxel, T. (2011). Poisson's ratio and modern materials. Nat. Mater., 10, 823-837.

Grenoble, D. E. \& Katz, J. L. (1971). The elastic constants of the constituent phases of dental amalgam. J. Biomed. Mater. Res., 5, 503-513.

Gruner, J. W. (1934). The crystal structures of talc and pyrophyllite. Z. Krist., 88, 412-419.

Gualtieri, A. F. (1999). Accuracy of XRPD QPA using the combined Rietveld-RIR method. J. Appl. Cryst., 33, 267-278.

Guazzato, M., Albakry, M., Ringer, S. \& Swain, M. (2004). Strength, fracture toughness and microstructure of a selection of all-ceramic materials. Part I. Pressable and alumina glassinfiltrated ceramics. Dent. Mater., 20, 441-448.

Guazzato, M., Albakry, M., Swain, M. V. \& Ironside, J. (2002). Mechanical properties of InCeram Alumina and In-Ceram Zirconia. Int. J. Prosthodont., 15, 339-346.

Gupta, K. (2002). Addenda to the Cr-Mo-Ni system (chromium-molybdenum-nickel). J. Phase Equilib., 23, 451-452.

Gupta, K. (2005). The Co-Cr-Mo (cobalt-chromium-molybdenum) system. J. Phase Equilib. Diffus., 26, 87-92.

Habelitz, S., Marshall Jr., G. W., Balooch, M. \& Marshall, S. J. (2002). Nanoindentation and storage of teeth. J. Biomech., 35, 995-998.

Hall, F. P. (1930). The influence of chemical composition on the physical properties of glazes. J. Am. Cer. Soc., 13, 182-199. 
Hanson, M. \& Pleva, J. (1991). The dental amalgam issue. A review. Experientia, 47, 9-22.

Harker, D. (1944). The crystal structure of $\mathrm{Ni}_{4}$ Mo. J. Appl. Phys., 12, 315-317.

Harmelin, M. (2006). The Landolt-Börnstein database: Thermodynamic properties - ternary alloy systems: Phase diagrams, crystallographic and thermodynamic data critically evaluated by MSIT ${ }^{\circledR}$ - noble metal systems. Selected systems from Ag-Al-Zn to Rh-Ru-Sc. Gold-Palladium - Platinum, volume 11B. Springer-Verlag.

Haussühl, S. (1993). Thermoelastic properties of beryl, topaz, diaspore, sanidine and periclase. Z. Krist., 204, 67-76.

Haussühl, S., Middendorf, B. \& Doerffel, M. (1991). Structure and properties of hopeites $\left(\mathrm{Mg}_{x} \mathrm{Zn}_{1-x}\right)_{3}\left(\mathrm{PO}_{4}\right)_{2} \cdot 4\left(\mathrm{H}_{2} \mathrm{O}\right)$. J. Sol. State Chem., 93, 9-16.

Hawthorne, F. C. \& Ferguson, R. B. (1975). Anhydrous sulphates. II. refinement of the crystal structure of anhydrite. Can. Min., 13, 289-292.

Henderson, B. \& Wilcox, R. J. M. (1964). Lattice spacing ralationships in hexagonal close-packed silver-zinc-manganese alloys. Philos. Mag., 9, 829-846.

Henderson, C. M. B. (1979). An elevated temperature x-ray study of synthetic disordered Na-K alkali feldspars. Contrib. Mineral. Petrol., 70, 71-79.

Hill, R. (1952). The elastic behaviour of a crystalline aggregate. Proc. Phy. Soc., A65, 349-354.

Höland, W., Apel, E., van't Hoen, C. \& Rheinberger, V. (2006). Studies of crystal phase formations in high-strength lithium disilicate glass-ceramics. J. Non-Cryst. Sol., 352, 4041-4050.

Huang, C.-C., Liu, Y.-C., Chen, L.-W. \& Chen, Y.-C. (2010). Temperature rise of alveolar bone during dental implant drilling using the finite element simulation. Life Science Journal, 12, $68-72$.

Hwa, L. G., Lee, T. H. \& Szub, S. P. (2004). Elastic properties of lanthanum aluminosilicate glasses. Materials Research Bulletin, 39, 33-40.

Hyson, J. M. (2006). Amalgam: Its history and perils. J. Calif. Dent. Assoc., 34, 215-229.

Ilie, N. (2004). Messmethoden zur Charakterisierung von Kompositfüllungswerkstoffen. Dissertation, Ludwig-Maximilian-Universität München, Poliklinik für Zahnerhaltung und Parodontologie.

Ilie, N., Bucuta, S. \& Draenert, M. (2013). Bulk-fill resin-based composites: An in vitro assessment of their mechanical performance. Oper. Dent., 38, In-Press.

Ipser, H., Mikula, A. \& Terzieff, P. (1983). Lattice parameters and melting behavior of the 
B2-phase in the systems $\mathrm{Cu}-\mathrm{Zn}, \mathrm{Ag}-\mathrm{Zn}, \mathrm{Au}-\mathrm{Zn}$ and Ag-Cd. Monatsh. Chem., 114, $1177-1184$.

Jang, J.-W., Kim, B. S., Kim, H. K. \& Lee, D. Y. (2004). Correlation between thermal expansion coefficients of $\mathrm{La}_{2} \mathrm{O}_{3}-\mathrm{Al}_{2} \mathrm{O}_{3}-\mathrm{SiO}_{2}$ glasses and strength of the glass infiltrated alumina for all ceramic crown. Mat. Sci. For., 449-452, 1193-1196.

Jette, E. R. \& Foote, F. (1935). Precision determination of lattice constants. J. Phys. Chem., 3, $605-616$.

Jong, B. d., Super, H., Spek, A., Veldman, N., Nachtegaal, G. \& Fischer, J. (1998). Mixed alkali systems: Structure and ${ }^{29} \mathrm{Si}_{\mathrm{MASNMR}}$ of $\mathrm{Li}_{2} \mathrm{Si}_{2} \mathrm{O}_{5}$ and $\mathrm{K}_{2} \mathrm{Si}_{2} \mathrm{O}_{5}$. Acta Cryst. B, 54, 568-577.

Jong, B. H. W. S. d., Slaats, P. G. G., Super, H. T. J., Veldman, N. \& Spek, A. L. (1994). Extended structures in crystalline phyllosilicates: silica ring systems in lithium, rubidium, cesium, and cesium/lithium phyllosilicate. J. Non-Cryst. Solids, 176, 164-171.

Kamann, W. K. (2000). Die Goldhämmerfüllung - Indikation und Technik. Schweiz Monatsschr. Zahnmed., 110, 597-606.

Kandil, S. H., El-Kady, A. S., El-Gamal, M. A. \& Morsi, S. E. (1989). Dynamic thermal expansion and scanning calorimetry of mercury phases in dental amalgams. J. Therm. Anal., 35, 21892197.

Käse, H. R. \& Tesk, J. A. (1989). Elastic constants of three Ni-Cr dental alloys at room and elevated temperatures. Dent. Mater., 5, 289-293.

Katz, J. L. \& Ukraincik, K. (1971). On the anisotropic elastic properties of hydroxyapatite. J. Biomech., 4, 221-227.

Kelly, J. R. (2008). Dental ceramics: What is this stuff anyway? J. Am. Dent. Assoc., 139 (suppl 4), 4S-7S.

Kelly, J. R., Nishimura, I. \& Campbell, S. D. (1996). Ceramics in dentistry: Historical roots and current perspectives. J. Prosth. Dent., 75, 18-32.

Kinney, J. H., Balooch, M., Marshall, G. W. \& Marshall, S. J. (1999). A micromechanics model of the elastic properties of human dentine. Arch. Oral Biol., 44, 813-822.

Kishen, A., Ramamurty, U. \& Asundi, A. (2000). Experimental studies on the nature of property gradients in the human dentine. J. Biomed. Mater. Res., 15, 650-659.

Kisi, E. \& Howard, C. J. (1998). Elastic constants of tetragonal zirconia measured by a new powder diffraction technique. J. Am. Ceram. Soc., 81, 1682-1684. 
Knosp, H., Holliday, R. J. \& Corti, C. W. (2003). Gold in dentistry: Alloys, uses and performance. Gold Bull., 36, 93-102.

Knosp, H., Nawaz, M. \& Stümke, M. (1981). Dental gold alloys. Gold Bull., 14, 57-64.

Kocks, U. F., Tomé, C. N. \& Wenk, H.-R. (2000). Texture and anisotropy - Preferred orientations in polycrystals and their effect on materials properties. Cambridge University Press.

Komura, Y., Sly, W. G. \& Shoemaker, D. P. (1960). The crystal structure of the R-phase, Mo-Co-Cr. Acta Cryst., 13, 575-585.

Kroll, H., Schmiemann, I. \& Cölln, G. (1986). Feldspar solid solutions. Am. Mineral., 71, 1-16.

Küster, Y., Leiss, B. \& Schramm, M. (2010). Structural characteristics of the halite fabric type 'kristallbrocken' from the Zechstein Basin with regard to its development. Int. J. Earth Sci. (Geol. Rundsch.), 99, $505-526$.

Kumagai, K., Nomura, N., Ono, T., Hotta, M. \& Chiba, A. (2005). Dry friction and wear behavior of forged Co-29Cr-6Mo alloy without $\mathrm{Ni}$ and $\mathrm{C}$ additions for implant applications. Mater. Trans., 46, 1578-1587.

Kumar, A., Jayakumar, T., Raj, B. \& Ray, K. K. (2003). Correlation between ultrasonic shear wave velocity and Poisson's ratio for isotropic solid materials. Acta Mater., 51, 2417-2426.

Larson, A. C. \& Van Dreele, R. B. (1994). General Structure Analysis System (GSAS). Los Alamos National Report LAUR, (pp. 86-748).

Le Bail, A. (1995). Modelling the silica glass structure by the Rietveld method. J. NonCryst. Sol., 183, 39-42.

Liebau, F. (1961). Untersuchungen an Schichtsilikaten des Formeltyps $\mathrm{A}_{m}\left(\mathrm{Si}_{2} \mathrm{O}_{5}\right)_{n}$. I. Die Kristallstruktur der Zimmertemperaturform des $\mathrm{Li}_{2} \mathrm{Si}_{2} \mathrm{O}_{5}$. Acta Cryst., 14, 389-395.

Low, D., Sumii, T. \& Swain, M. (2001). Thermal expansion coefficient of titanium casting. J. Oral Rehabil., 28, 239-242.

Lutterotti, L., Ceccato, R., Dal Maschio, R. \& Pagani, E. (1998). Quantitative analysis of silicate glasses by the Rietveld method. Mat. Sci. For., 278-281, 87-92.

Lutterotti, L., Chateigner, D., Ferrari, S. \& Ricote, J. (2004). Texture, residual stress and structural analysis of thin films using a combined x-ray analysis. Thin Solid Films, 450, 34-41.

Lutterotti, L., Matthies, S., Wenk, H.-R., Schultz, A. J. \& Richardson, J. W. (1997). Combined texture and structure analysis of deformed limestone from time-of-flight neutron diffraction spectra. J. Appl. Phys., 81, 594-600. 
Mahmoud, A., Wakabayashi, N., Takahashi, H. \& Ohyama, T. (2005). Deflection fatigue of Ti-6Al-7Nb, Co-Cr and gold alloy cast clasps. J. Prosthet. Dent., 93, 183-188.

Maistrelli, P., Lutterotti, L. \& Scardi, P. (1994). Thermal behavior of monoclinic zirconia at low temperature by XRPD full pattern analysis. Mat. Sci. For., 166-169, 495-500.

Massalski, T. B. (1990). Binary Alloys Phase Diagram. ASM International, 2. Auflage.

Mastelaro, V. R. \& Zanotto, E. D. (1999). Anisotropic residual stresses in partially crystallized $\mathrm{Li}_{2} \mathrm{O}-2 \mathrm{SiO}_{2}$ glass-ceramics. J. Non-Cryst. Solids, 247, 79-86.

Matsuo, Y. \& Nagasava, A. . K. J. (1966). Ordered alloys of the gold-palladium system. II. Electron diffraction study on evaporated $\mathrm{AuPd}_{3}$ films. J. Phys. Soc. Jap., 21, 2633-2637.

Matthies, S. \& Vinel, G. W. (1982). On the reproduction of the orientation distribution function of textured samples from reduced pole figures using the concept of conditional ghost correction. Phys. Status Solidi B, 112, K111-K114.

Matthies, S. \& Wenk, H.-R. (2009). Transformations for monoclinic crystal symmetry in texture analysis. J. Appl. Cryst., 42, 564-571.

McCracken, M. (1999). Dental implant materials: Commercially pure titanium and titanium alloys. J. Prosthodont., 8, 40-43.

Meneghinello, E., Alberti, A. \& Cruciani, G. (1999). Order-disorder process in the tetrahedral sites of albite. Am. Mineral., 84, 1144-1151.

Mohsen, C. (2011). Corrosion effect on the flexural strength \& micro-hardness of ips e-max ceramics. Open J. Stomatology, 1, 29-35.

Moseley, H. G. J. (1913). The high-frequency spectra of the elements. Phil. Mag., 26, 1024-1034.

Murphy, A. J. (1931). The constitution of the alloys of silver and mercury. J. Inst. Met., 46, $507-522$.

Nanci, A. (2008). Ten Cate's Oral Histology. Mosby Elsevier, 7. Auflage.

Naray-Szabo, S. (1936). Zur Struktur des Baddeleyits $\mathrm{ZrO}_{2}$. Z. Krist., 94, 414-416.

Negro, A. d., Pieri, R. d. \& Quareni, S. (1978). The crystal structures of nine K feldspars from the Adamello Massif (Northern Italy). Acta Cryst. B, 34, 2699-2707.

Nomura, T., Katz, J. L., Powers, M. P. \& Saito, C. (2007). A micromechanical elastic property study of trabecular bone in the human mandible. J. Mater. Sci.: Mater. Med., 18, 629-633. 
Nono, M. C. A. (2005). Tetragonal-to-monoclinic transformation influence on the mechanical properties of $\mathrm{CeO}_{2}-\mathrm{ZrO}_{2}$ ceramics. J. Mat. Sci., 498-499, 506-511.

Ohno, I., Yamamoto, W. \& Anderson, O. L. (1986). Determination of elastic constants of trigonal crystals by the rectangular parallelepiped resonance method. J. Phys. Chem. Solids, 47, 11031108 .

Okrusch, M. \& Matthes, S. (2005). Mineralogie, eine Einführung in die spezielle Mineralogie, Petrologie und Lagerstättenkunde. Springer, 7. Auflage.

Oliver, W. C. \& Pharr, G. M. (1992). An improved technique for determining hardness and elastic modulus using load and displacement sensing indentation experiments. J. Mater. Res., $7,1564-1583$.

Palmer, D. C., Dove, M. T., Ibberson, R. M. \& Powell, B. M. (1997). Structural behavior, crystal chemistry, and phase transitions in substituted leucite: High-resolution neutron powder diffraction studies. Am. Mineral., 82, 16-29.

Patterson, G. (1976). Light scattering and the local structure of amorphous polymers. $J$. $M a-$ cromol. Sci.-Phys. B, 12, 61-74.

Pawar, R. R. \& Deshpande, V. T. (1968). The anisotropy of the thermal expansion of $\alpha$-titanium. Acta Cryst., A24, 316-317.

Pecharsky, V. K. \& Zavalij, P. Y. (2005). Fundamentals of Powder Diffraction and structural characterization of materials. Springer.

Perdikatsis, B. \& Burzlaff, H. (1981). Strukturverfeinerung am Talk $\mathrm{Mg}_{3}\left((\mathrm{OH})_{2} \mathrm{Si}_{4} \mathrm{O}_{10}\right)$. Z. Krist., 156, 177-186.

Pharr, G. M., Herbert, E. G. \& Gao, Y. (2010). The indentation size effect: A critical examination of experimental observations and mechanistic interpretations. Annu. Rev. Mater. Res., 40, $271-92$.

Piconi, C. \& Maccauro, G. (1999). Zirconia as a ceramic biomaterial - review. Biomaterials, 20, $1-25$.

Pieniak, A., Niewczas, A. M. \& Kordos, P. (2012). Influence of thermal fatigue and ageing on the microhardness of polymer-ceramic composites for biomedical applications. Maintenance and Reliability, 14, 181-188.

Preston, G. D. (1931). The x-ray examination of the system silver-mercury. J. Inst. Met., 46, $522-527$.

Prince, A. (2006). The Landolt-Börnstein Database: Thermodynamic Properties - Ternary Alloy Systems: Phase Diagrams, Crystallographic and Thermodynamic Data critically evaluated by 
MSIT®- Noble Metal Systems. Selected Systems from Ag-Al-Zn to Rh-Ru-Sc. Silver - GoldPlatinum, volume 11B. Springer-Verlag.

Prinz, H. (1923). Pierre Fauchard and his works. Dent. Cosmos, 65, 827-830.

Pröbster, L. \& Groten, M. (2008). VITA Vollkeramik VITA In-Ceram: Leitfaden für vollkeramische Restaurationen in der zahnärztlichen Praxis. 3. Auflage.

Raue, L. (2012). Private Kommunikation.

Raue, L. (2013). Private Kommunikation.

Raue, L. \& Klein, H. (2011). Calculation of anisotropic properties of dental enamel from synchrotron data. J. Synchrotron Rad., 18, 550-556.

Raue, L., Klein, H. \& Hartmann, C. (2011). Elastic modulus of human dental enamel from different methods. Int. J. Biomater. Res. Eng., 1, 39-48.

Reddy, J. J., Chakraborty, A. \& Bhaduri, S. B. (1989). Thermal expansion behaviour of $\mathrm{ZrO}_{2}-12$ $\mathrm{mol} \% \mathrm{CeO}_{2}$ and $\mathrm{ZrO}_{2}-20 \mathrm{~mol} \% \mathrm{CeO}_{2}$ materials. J. Mat. Sci. Lett., 8, 210-2011.

Reuss, A. (1929). Berechnung der Fließgrenze von Mischkristallen auf Grund der Plastizitätsbedingung für Einkristalle. Z. Angew. Math. Mech., 9, 49-58.

Rietveld, H. M. (1967). Line profiles of neutron powder-diffraction peaks for structure refinement. J. Appl. Cryst., 22, 151-152.

Roberts, W. T. (1962). Preferred orientation and anisotropy in titanium. J. Less Common Metals, 4, 345-361.

Rüdinger, K. (1978). Moderne Werkstoffe - Auswahl - Prüfung - Anwendung: Titan und Titanlegierungen. Z. Werkstofftech., 9, 181-188.

Rudman, P. (1967). Lattice parameters of some hcp binary alloys of rhenium and osmium: Re-W, Re-Ir, Re-Pt, Os-Ir, Os-Pt. J. Less-Common Met., 12, 79-81.

Rueggeberg, F. A. (2002). From vulcanite to vinyl, a history of resins in restorative dentistry. J. Prosth. Dent., 87, 364-379.

Saenger, A. T. \& Kuhs, W. F. (1992). Structural disorder in hydroxyapatite. Z. Krist., 199, $123-148$.

Sakaguchi, R. L. \& Powers, J. M. (2012). Craig's restorative dental materials. Elsevier Mosby, 13. Auflage.

Santana, D. P., Pion de Carvalho, A. 1., Andrade Pizani, A. M., Coury Saraceni, C. H. \& Queiroz, 
C. S. (2010). Evaluation of microhardness on polymerization's photo of resin composites by halogen light and light emitting diode. Odontol. Clin.-Cient., 9, 239-242.

Sawada, H. (1994). Residual electron density study of alpha-aluminium oxide through refinement of experimental atomic scattering factors. Mater. Res. Bull., 29, 127-133.

Sawada, H. \& Takeuchi, Y. (1990). The crystal structure of barite, beta-BaSO ${ }_{4}$, at high temperatures. Z. Krist., 191, 161-171.

Scardi, P., Di Maggio, R. \& Lutterotti, L. (1992). Thermal expansion anisotropy of ceriastabilized tetragonal zirconia. J. Am. Ceram. Soc., 75, 2828-2832.

Schmage, P., Nergiz, I., Sito, F., Platzer, U. \& Rosentritt, M. (2009). Wear and hardness of different core build-up materials. J. Biomed. Mat. Res. Part B: App. Biomat., 91, 71-79.

Schmitt, L., Lurtz, C., Behrend, D. \& Schmitz, K.-P. (2008). Registered microhardness of human teeth parts and dental filling composites. IFMBE Proceedings, 22, 2252-2254.

Seong, W.-J., Kim, U.-K., Swift, J. Q., Heo, Y.-C., Hodges, J. S. \& Ko, C.-C. (2009). Elastic properties and apparent density of human edentulous maxilla and mandible. Int. J. Oral Maxillofac. Surg., 38, 1088-1093.

Shannon, R. D. (1976). Revised effective ionic radii and systematic studies of interatomic distances in halides and chalcogenides. Acta Cryst. A, 32, 751-767.

Shvyd'ko, Y. V., Lucht, M., Gerdau, E., M., L., Alp, E. E., Sturhahn, W., Sutter, J. \& Toellner, T. S. (2002). Measuring wavelengths and lattice constants with the Mössbauer wavelength standard. J. Synchrotron Rad., 9, 17-23.

Southan, D. E. (1975). Dental Porcelain. In: Scientific aspects of dental materials. London: Butterworths.

Spielmann, A. I. (2007). The birth of the most important 18th century dental text: Pierre Fauchard's Le Chirurgien Dentiste . J. Dent. Res., 86, 922-926.

Spiess, M. \& Gruehn, R. (1979). Zur thermischen Dehydratisierung des $\mathrm{ZnSO}_{4} \cdot 7 \mathrm{H}_{2} \mathrm{O}$ und zum Hochtemperaturverhaltem von wasserfreiem $\mathrm{ZnSO}_{4}$. Z. Anorg. Allg. Chem., 456, 222-240.

Staines, M., Robinson, W. H. \& Hood, J. A. A. (1981). Spherical indentation of tooth enamel. J. Mater. Sci., 16, 2551-2556.

Stollwerk, K. (2009). Postendodontischer Aufbau von Zähnen mit FRC-Stiften - Ergebnisse einer prospektiven klinischen Studie. Dissertation, Medizinische Fakultät der RheinischWestfälischen Technischen Hochschule Aachen. 
Straumanis, M. E. \& Weng, C. C. (1955). The precise lattice constant and the expansion coefficient of chromium between +10 and $+60^{\circ} \mathrm{C}$. Acta Cryst., 8, 367-371.

Swanson, H. E. \& Tatge, E. (1953). Standard x-ray diffraction powder patterns. National Bureau of Standards (U.S.), Circular, 359, 95.

Taggart, W. H. (1907). A new and accurate method of casting gold inlays. Dent. Cosmos, 49, $1117-1121$.

Tait, K. T., Sokolova, E., Hawthorne, F. C. \& Khomyakov, A. P. (2003). The crystal chemistry of nepheline. Can. Mineral., 41, 61-70.

Tani, T., Udoh, K., Yasuda, K., Van Tendeloo, G. \& Van Landuyt, J. (1991). Age-hardening mechanisms in a commercial dental gold alloy containing platinum and palladium. J. Dent. Res., 70, 1350-1357.

Thiel, S. \& Schnapp, J. (1998). Anisotropic crack extension in aligned glass ceramic. J. NonCryst. Solids, 242, 189-194.

Toby, B. H. (2001). EXPGUI, a graphical user interface for GSAS. J. Appl. Cryst., 34, 210-213.

Torre, A. G. d. 1., Bruque, S. \& Aranda, A. G. (2001). Rietveld quantitative amorphous content analysis. J. Appl. Cryst., 34, 196-202.

Tromans, D. (2011). Elastic anisotropy of hcp metal crystals and polycrystals. Int. J. Res. Rev. Appl Sci., 6, 462-483.

Tseng, H.-Y., Heaney, P. J. \& Onstott, T. C. (1995). Characterization of lattice strain induced by neutron irradiation. Phys. Chem. Minerals, 22, 399-405.

Uçar, Y. \& Brantley, W. A. (2011). Biocompatibility of dental amalgams. Int. J. Dent., 2011, 7 pages.

Ullner, C. (2004). Die Reihe DIN EN ISO 14577 - Erste weltweit akzeptierte Normen für die instrumentierte Eindringprüfung. DGM-Tagungsband "Werkstoffprüfung". 11-20.

Vedel, I., Redon, A. M., Rossat-Mignod, J., Vogt, O. \& Leger, J. M. (1987). Electronic and crystallographic transitions induced by pressure in CeP. J. Phys. Chem. C, 20, 3439-3444.

VIDENT (2002). VITABLOCS MARK II for CEREC: Materials science and clinical studies. Bericht.

Völlenkle, H. (1981). Verfeinerung der Kristallstrukturen von $\mathrm{Li}_{2} \mathrm{SiO}_{3}$ und $\mathrm{Li}_{2} \mathrm{GeO}_{3}$. Z. Krist., $154,77-81$.

Voigt (1928). Lehrbuch der Kristallphysik. Leipzig: Teubner. 
Wang, D.-N., Guo, Y.-Q., Liang, K.-M. \& Tao, K. (1999). Crystal structure of zirconia by Rietveld refinement. Sci. China A: mathematics, 42, 80-86.

Wang, Y. N. \& Huang, J. C. (2003). Texture analysis in hexagonal materials. Mater. Chem. Phys., 81, 11-26.

Washburn, E. W. (1930). International Critical Tables of Numerical Data, Physics, Chemistry and Technology, Vol. III. McGraw-Hill.

Waszkiel, D. (1990). Microhardness of amalgams stabil b and amalcap. Czas Stomatol., 43, 587-591. Abstract. Artikel auf Polnisch.

Weiler, W. (1990). Zusammenhang zwischen Vickershärte und Universalhärte. Materialprüfung, $32,149-151$.

Wenk, H.-R., Matthies, S., Donovan, J. \& Chateigner, D. (1998). BEARTEX, a Windows-based program system for quantitative texture analysis. J. Appl. Cryst., 31, 262-26.

Wildner, M. \& Giester, G. (1988). Crystal structure refinements of synthetic chalcocyanite $\left(\mathrm{CuSO}_{4}\right)$ and zincosite $\left(\mathrm{ZnSO}_{4}\right)$. Mineral. Petrol., 39, 201-209.

Wildner, M. \& Giester, G. (1991). The crystal structures of kieserite-type compounds. i. crystal structures of $\mathrm{Me}(\mathrm{II}) \mathrm{SO}_{4} \cdot \mathrm{H}_{2} \mathrm{O}(\mathrm{Me}=\mathrm{Mn}, \mathrm{Fe}, \mathrm{Co}, \mathrm{Ni}, \mathrm{Zn})$. N. Jb. Min., 7, 296-306.

Willaime, C., Bnow, L. \& Perucaud, M. C. (1974). On the orientation of the thermal and compositional strain ellipsoids in feldspars. Am. Mineral., 59, 457-464.

Winter, J. K., Okamura, F. P. \& Ghose, S. (1979). A high temperature structural study of high albite, monalbite, and the analbite-monalbite phase transition. Am. Mineral., 64, 409-423.

Wolfenden, A. \& Hood, J. A. A. (1980). Young's modulus and mechanical damping of silver dental alloys. J. Mater. Sci., 15, 2995-3002.

Wood, R. (1962). The lattice constants of high purity alpha titanium. Proc. Phys. Soc. London, 80,783 .

Xu, H., Liu, W. \& Wang, T. (1989). Measurement of thermal expansion coefficient of human teeth. Aust. Dent. J., 34, 530-535.

Yakubovich, O. V. \& Urosova, V. S. (1997). Electron distribution in lithiophosphatite: Crystallochemical features of orthophosphates with hexagonal close packing. Kristallografiya, 42, 301-308.

Yamaguchi, H., Akatsuka, K. \& Setoguchi, M. (1979). Structure of dilithium zinc silicate $\gamma_{I I^{-}}$ $\mathrm{Li}_{2} \mathrm{ZnSiO}_{4}$. Acta Cryst. B, 35, 2678-2680. 
Yasudo, K. (1987). Age-hardening and related phase transformations in dental gold alloys. Gold Bull., 20, 90-103.

Young, R. A. \& Spooner, S. (1969). Neutron diffraction studies of human tooth enamel. Archs. Oral. Biol., 15, 47-63.

Zhurova, E. A., Maximov, B. A., Simonov, V. I. \& Sobolev, B. P. (1996). Structural studies of $\mathrm{CaF}_{2}\left(\right.$ at $296 \mathrm{~K}$ ) and $\mathrm{Ca}_{1-x} \operatorname{Pr}_{x} \mathrm{~F}_{2+x}, \mathrm{x}=0.1$ (at 296 and $170 \mathrm{~K}$ ) crystals. The changes in the fluorite anionic motif in the partial substitution of $\mathrm{Ca}(2+)$ by $\operatorname{Pr}(3+)$ cations. Kristallografiya, $41,438-443$. 
A TABELLEN

Anhang

A Tabellen 
Tab. A.1: Chemische Zusammensetzung der Dentalkomposite gemäß Herstellerangaben.

\begin{tabular}{|c|c|c|c|c|}
\hline Firma & $\begin{array}{l}\text { Handels- } \\
\text { name }\end{array}$ & $\begin{array}{l}\text { Organische } \\
\text { Komponente }\end{array}$ & $\begin{array}{l}\text { Anorganische } \\
\text { Füllkörper }\end{array}$ & $\begin{array}{l}\text { Füllkörper- } \\
\text { größe }\end{array}$ \\
\hline DMG & $\begin{array}{l}\text { Luxacore } \\
\text { dual }\end{array}$ & Bis-GMA ${ }^{1} \mathrm{UDMA}^{2}$ & $\begin{array}{l}\text { Bariumglas, pyrogene } \\
\text { Kieselsäure }\end{array}$ & $0,02-2,4 \mu \mathrm{m}$ \\
\hline VOCO & $\begin{array}{l}\text { Rebilda } \\
L C\end{array}$ & $\begin{array}{l}\text { Diurethandimethacrylat, } \\
\text { Bis-GMA, BHT, TEG- } \\
\text { DMA }^{3}\end{array}$ & Ba-B-Al-SiO ${ }_{2}$-Glas & k.A. \\
\hline $\mathrm{VOCO}$ & $\begin{array}{l}\text { Rebilda } \\
D C\end{array}$ & $\begin{array}{l}\text { Diurethandimethacrylat, } \\
\text { BIS-GMA, BHT, } \\
\text { Benzoylperoxid }\end{array}$ & Ba-B-Al-SiO ${ }_{2}$-Glas & k.A. \\
\hline $\begin{array}{l}\text { Ivoclar } \\
\text { vivadent }\end{array}$ & Tetric & $\begin{array}{l}\text { Bis-GMA, UDMA, } \\
\text { TEGDMA }^{3}\end{array}$ & Ba-Glas, $\mathrm{YbF}_{3}$, Mischoxid & $\begin{array}{l}0,04-3 \mu \mathrm{m} \\
(\varnothing=0,55 \mu \mathrm{m})\end{array}$ \\
\hline $\begin{array}{l}\text { Ivoclar } \\
\text { vivadent }\end{array}$ & $\begin{array}{l}\text { Tetric Evo } \\
\text { Flow }\end{array}$ & $\begin{array}{l}\text { Bis-GMA, UDMA } 2 \\
\text { Decandioldimethacrylat }\end{array}$ & $\begin{array}{l}\text { Ba-Glas, } \mathrm{YbF}_{3}, \quad \text { Misch- } \\
\text { oxid, hoch disperses } \mathrm{SiO}_{2}\end{array}$ & $\begin{array}{l}0,04-3 \mu \mathrm{m} \\
(\varnothing=0,55 \mu \mathrm{m})\end{array}$ \\
\hline $\begin{array}{l}\text { Ivoclar } \\
\text { vivadent }\end{array}$ & $\begin{array}{l}\text { Tetric } \\
\text { Ceram }\end{array}$ & $\begin{array}{l}\text { Bis-GMA, UDMA }{ }^{2} \text { Bis- } \\
\text { EMA }^{4}\end{array}$ & Ba-Glas, $\mathrm{YbF}_{3}$, Mischoxid & $\begin{array}{l}0,04-3 \mu \mathrm{m} \\
(\varnothing=0,55 \mu \mathrm{m})\end{array}$ \\
\hline $\mathrm{VOCO}$ & Clip & $\begin{array}{l}\text { HEMA } 6 \text { BHT, }{ }^{5} \text { Acrylat- } \\
\text { ester, Polymere }\end{array}$ & - & - \\
\hline Dentsply & $\operatorname{CeramX}$ & $\begin{array}{l}\text { Dimethacrylate, } \\
\text { Methacrylat-modifiziertes } \\
\text { Polysiloxan }\end{array}$ & $\begin{array}{l}\text { Ba-B-Al-SiO }{ }_{2} \text {-Glas, amor- } \\
\text { phes } \mathrm{SiO}_{2}\end{array}$ & Nanofüller \\
\hline $\begin{array}{l}\text { Heraeus } \\
\text { Kulzer }\end{array}$ & Venus C3 & BIS-GMA를asis & $\begin{array}{l}\text { Ba-Al-F-Glas, hoch dis- } \\
\text { perses } \mathrm{SiO}_{2}\end{array}$ & $\begin{array}{l}\emptyset=0,7 \mu \mathrm{m} \\
(\mathrm{Ba}-\mathrm{Al}-\mathrm{F}-\mathrm{Glas}), \\
\emptyset=0,04 \mu \mathrm{m} \\
(\text { amorphes } \\
\left.\mathrm{SiO}_{2}\right)\end{array}$ \\
\hline $\mathrm{VOCO}$ & Clip & $\begin{array}{l}\text { HEMA, BHT, }{ }^{5} \text { Acrylat- } \\
\text { ester, Polymere }\end{array}$ & - & - \\
\hline $\mathrm{VOCO}$ & $\begin{array}{l}\text { Cimara } \\
\text { Zircon, } \\
\text { Grandioso }\end{array}$ & $\begin{array}{l}\text { Methacrylatmatrix, BIS- } \\
\text { GMA }^{1}\end{array}$ & $\begin{array}{l}90 \text { Gew } \% \text { anorganische } \\
\text { Füllstoffe }\end{array}$ & k.A. \\
\hline
\end{tabular}

${ }^{1}$ Bis-GMA: Bisphenol A-Glycidylmethacrylat

${ }^{2}$ UDMA: Urethandimethacrylat

3 TEG-DMA: Triethylenglykol-Dimethacrylat

${ }^{4}$ BIS-EMA: Ethoxyliertes Bisphenol A-Dimethacrylat

${ }^{5}$ BHT: Butylhydroxytoluol

${ }^{6}$ HEMA: Hydroxyethylmethacrylat 
Tab. A.2: Chemische Zusammensetzung von Glasionomer- und Zn-Sulfatzementen in ihrem Ausgangszustand gemäß Herstellerangaben.

\begin{tabular}{lll}
\hline \hline Firma & Handelsname & Chemische Zusammensetzung [Gew.\%] \\
\hline 3M ESPE & Ketac Fil plus & $\begin{array}{l}\text { Glaspulver, Wasser }(50-65), \text { Polyacrylsäure-co-Maleinsäure } \\
(30-45), \text { Weinsäure }(5-10)^{1}\end{array}$ \\
\hline Detax & Detaferm & Zinkoxid $(20-40)$, Zinksulfat $(<10)$, Calciumfluorid $(<0,3)$ \\
\hline 3M ESPE & Cavit W & $\begin{array}{l}\text { Calciumsulfat }(1-30), \text { Bariumsulfat }(0-20), \text { Talk }(0-20), \\
\text { Polyvinyl-Acetat }(1-5), \text { Zinksulfat }(5-10), \text { Triethylenglycol- } \\
\text { diacetat }(10-20)\end{array}$ \\
\hline \hline
\end{tabular}

${ }^{1}$ Prozentuale Angaben der Massenanteile beziehen sich nur auf die Flüssigkeit.

Tab. A.3: Chemische Zusammensetzung der Prothesenkunststoffe gemäß Herstellerangaben.

\begin{tabular}{|c|c|c|}
\hline Firma & Handelsname & Chemische Zusammensetzung \\
\hline Degudent & Dentsply-Bioplus & $\begin{array}{l}\text { interpenetriertes Silikon-Copolymer-Netzwerk, keine } \\
\text { anorganischen Füllstoffe }\end{array}$ \\
\hline Weithas & Weitur-Press & Polymer auf Methylmethacrylat-Basis \\
\hline Kulzer & Pala-Press & Methylmethacrylat-Copolymer, Dimethacrylat \\
\hline Candulor & Aesthetic & $\begin{array}{l}\text { Polymethylmethacrylat, Dimethacrylat, Weich- } \\
\text { macher, Benzoylperoxid, Katalysator, Pigmente, } \\
\text { Reyonfasern }\end{array}$ \\
\hline Candulor & $\begin{array}{l}\text { Aesthetic } \\
\text { Faseranteil) }\end{array}$ & $\begin{array}{l}\text { Polymethylmethacrylat, } \quad \text { Dimethacrylat, Weich- } \\
\text { macher, Benzoylperoxid, Katalysator, Pigmente, } \\
\text { Reyonfasern }\end{array}$ \\
\hline
\end{tabular}

Tab. A.4: Chemische Zusammensetzung von Cercon Base gemäß Herstellerangaben.

\begin{tabular}{cc}
\hline \hline Oxid & Anteil in Gew.\% \\
\hline $\mathrm{ZrO}_{2}$ & $>92$ \\
$\mathrm{Y}_{2} \mathrm{O}_{3}$ & 5 \\
$\mathrm{HfO}_{2}$ & $<2$ \\
$\mathrm{Al}_{2} \mathrm{O}_{3} / \mathrm{SiO}_{2}$ & $<1$ \\
\hline \hline
\end{tabular}


Tab. A.5: Chemische Zusammensetzung der Hauptphasen der Dentalkeramik Vitablocs Esthetic Line aus EDX-Analysen. Standardabweichungen der Einzelanalysen vom Mittelwert sind in Klammern angegeben.

\begin{tabular}{|c|c|c|c|c|c|c|c|c|}
\hline Phase & Einheit & $\mathrm{Na}$ & $\mathbf{K}$ & $\mathbf{C a}$ & $\mathrm{Al}$ & $\mathrm{Si}$ & Summe & $\mathrm{n}^{1}$ \\
\hline \multirow{2}{*}{ Glasmatrix } & Gew.\% & $7,3(4)$ & $9(4)$ & $3(5)$ & $21(1)$ & $60(2)$ & 100,0 & 6 \\
\hline & $\mathrm{apfu}^{2}$ & $0,45(3)$ & $0,40(1)$ & $0,03(3)$ & $1,07(5)$ & $2,97(8)$ & $4,9(2)$ & 6 \\
\hline \multirow{2}{*}{ Sanidin } & Gew.\% & $5,8(8)$ & $16(14)$ & $2(5)$ & $18(5)$ & $58(11)$ & 99,9 & 14 \\
\hline & $\mathrm{apfu}^{2}$ & $0,35(5)$ & $0,50(6)$ & $0,01(1)$ & $0,99(3)$ & $3,04(2)$ & $4,9(2)$ & 14 \\
\hline \multirow{2}{*}{ Nephelin ${ }^{3}$} & Gew.\% & $16,6(9)$ & $5,9(5)$ & $2,76(1)$ & $32,5(9)$ & $42(1)$ & 100,0 & 2 \\
\hline & $\mathrm{apfu}^{2}$ & $1,09(5)$ & $0,23(2)$ & $0,103(2)$ & $1,81(4)$ & $2,26(7)$ & $5,5(2)$ & 2 \\
\hline Albit-reicher & Gew.\% & $10,6(5)$ & $5(1)$ & $0,5(1)$ & $19,6(5)$ & $64,41(3)$ & 100,0 & 2 \\
\hline Felspat & $\mathrm{apfu}^{2}$ & $0,62(2)$ & $0,17(4)$ & $0,016(2)$ & $0,97(2)$ & $3,07(1)$ & $4,84(9)$ & 2 \\
\hline
\end{tabular}

${ }^{1}$ Zahl der Einzelanalysen.

${ }^{2}$ apfu (engl. für atoms per formula unit): Atome pro Formeleinheit bei Normierung der Kationenzahl auf 8 Sauerstoffatome.

3 Aufgrund der geringen Korngröße liegen hier sehr wahrscheinlich Mischanalysen von Nephelin mit Feldspat vor.

Tab. A.6: Ergebnisse der standardfreien EDX-Analyse am REM für die Dentalkeramik IPS e.max Press im Rohzustand. Prozentangaben beziehen sich auf $100 \%$ der detektierbaren Elemente. Standardabweichungen sind in Klammern gegeben (Mittelwerte aus 5 Einzelanalysen).

\begin{tabular}{lcc}
\hline \hline Element (Linie) & Anteile in Gew.\% & Anteile in At.\% \\
\hline $\mathrm{Si}(\mathrm{Si}-\mathrm{K})$ & $72,3(1,3)$ & $80(1,1)$ \\
$\mathrm{Zn}(\mathrm{Zn}-\mathrm{K})$ & $7,6(0,8)$ & $3,6(0,4)$ \\
$\mathrm{K}(\mathrm{K}-\mathrm{K})$ & $6,8(0,3)$ & $5,4(0,2)$ \\
$\mathrm{P}(\mathrm{P}-\mathrm{K})$ & $3,8(0,2)$ & $3,8(0,2)$ \\
$\mathrm{Ce}(\mathrm{Ce}-\mathrm{L})$ & $3,7(0,2)$ & $0,81(0,04)$ \\
$\mathrm{Na}(\mathrm{Na}-\mathrm{K})$ & $3,2(0,3)$ & $4,3(0,4)$ \\
$\mathrm{Al}(\mathrm{Al}-\mathrm{K})$ & $1,6(0,1)$ & $1,9(0,1)$ \\
$\mathrm{Yb}(\mathrm{Yb}-\mathrm{L})$ & $0,7(0,5)$ & $0,1(0,1)$ \\
$\mathrm{Fe}(\mathrm{Fe}-\mathrm{K})$ & $0,4(0,3)$ & $0,2(0,2)$ \\
\hline \hline
\end{tabular}

Tab. A.7: Verfeinerte Gitterparameter der Amalgam-Phasen AgSn, $\mathbf{A g}_{2} \mathbf{H g}_{3} \mathbf{u n d}_{\mathbf{C u}} \mathrm{Cn}_{5}$ in extrahiertem Zahn. Daten an der Beamline BW5 $(\lambda=0,1214 \AA)$ und mit GSAS ausgewertet.

\begin{tabular}{l|ccc}
\hline \hline Phase (Struktur) & $a(\AA)$ & $b(\AA)$ & $c(\AA)$ \\
\hline $\mathrm{AgSn}(P m m n)$ & $6,001(5)$ & $4,831(1)$ & $5,221(4)$ \\
$\mathrm{Ag}_{2} \mathrm{Hg}_{3}(I 23)$ & $10,060(2)$ & $10,060(2)$ & $10,060(2)$ \\
$\mathrm{Cu}_{6} \mathrm{Sn}_{5}\left(P 6_{3} / m m c\right)$ & $4,218(3)$ & $4,218(3)$ & $5,122(6)$ \\
\hline \hline
\end{tabular}


Tab. A.8: Verfeinerte Gitterparameter für die Silikat-Phasen der Keramik Vitablocs - Esthetic Line. Rietveld-Verfeinerung anhand eines Datensatzes an einem CAD/CAM-Block gemessen an der Beamline P02.1 (PETRA; $\lambda \approx 0,2088 \AA$ ) - Auswertung mit MAUD.

\begin{tabular}{l|ccc}
\hline \hline Mineral & Nephelin & Sanidin & Mikroklin \\
\hline $\mathrm{a}(\AA)$ & $10,02073(5)$ & $8,2580(2)$ & $8,4241(1)$ \\
$\mathrm{b}(\AA)$ & $10,02073(5)$ & $13,0623(3)$ & $12,8027(2)$ \\
$\mathrm{c}(\AA)$ & $8,37110(6)$ & $7,2568(2)$ & $7,0787(1)$ \\
$\alpha\left(^{\circ}\right)$ & 90 & 90 & $91,335(2)$ \\
$\beta\left(^{\circ}\right)$ & 90 & $117,127(2)$ & $114,492(1)$ \\
$\gamma\left({ }^{\circ}\right)$ & 120 & 90 & $87,273(1)$ \\
\hline \hline
\end{tabular}

Tab. A.9: Ergebnisse der Phasenanalyse für die Korund-Keramik Vita In-Ceram Alumina im vor- und endgesinterten Zustand ohne Berücksichtigung des Glasanteils. Geschätzte Standardabweichungen aus der Rietveld-Analyse sind in Klammern angegeben. Mittelwerte der einzelnen Phasenanalysen sind fettgedruckt.

\begin{tabular}{l|cccccc}
\hline \hline Zustand & $R_{w p}$ & Kor und & Spinell & $\mathbf{n}^{\mathbf{1}}$ & Beamline & Programm \\
\hline Block 1 (vorgesintert) & 2,92 & $99,16(4)$ & $0,84(6)$ & 1 & BW5 & GSAS \\
Block 1 (vorgesintert) & 14,33 & $99,14(26)$ & $0,856(5)$ & $1^{*} 41$ & BW5 & MAUD \\
Scheibe (vorgesintert) & 11,87 & $99,00(20)$ & $1,00(3)$ & 1 & BW5 & MAUD \\
Block 2 (vorgesintert) & 27,41 & $98,91(16)$ & $1,09(1)$ & $1^{*} 41$ & BW5 & MAUD \\
Mittelwert (vorgesinterte Keramik) & & $\mathbf{9 9 , 0 5 ( 1 2 )}$ & $\mathbf{0 , 9 5 ( 1 2 )}$ & & & \\
\hline Scheibe (La-Glas infiltriert) & 15,41 & 100,00 & 0,00 & $1^{*} 41$ & BW5 & MAUD \\
Scheibe (La-Glas infiltriert) & 3,3 & 100,00 & 0,00 & 1 & W2 & GSAS \\
\hline \hline
\end{tabular}

${ }^{1}$ Zahl der aufgenommenen Beugungsbilder $\left(1=\right.$ Einzelaufnahme; $1^{*} 41=1$ Positionshöhe mit 41 Beugungsbildern, die bei Drehung der Probe um $\omega$ in $4^{\circ}$-Schritten von $-80^{\circ}$ bis $+80^{\circ}$ aufgenommen wurden.)

Tab. A.10: Ergebnisse der Phasenanalyse für die Korund-Keramik Vita In-Ceram Zirkonia im vor- und endgesinterten Zustand ohne Berücksichtigung des Glasanteils. Geschätzte Standardabweichungen aus der Rietveld-Analyse sind in Klammern angegeben. Mittelwerte der einzelnen Phasenanalysen sind fettgedruckt.

\begin{tabular}{l|ccccccc}
\hline \hline Zustand & $R_{w p}$ & Korund & $\mathbf{C e Z r O}_{\mathbf{2}} \mathbf{- t}$ & $\mathbf{C e Z r O}_{\mathbf{2}} \mathbf{- m}$ & $\mathbf{n}^{\mathbf{1}}$ & Beamline & Programm \\
\hline Block 1 (vorgesintert) & 6,31 & $65,09(20)$ & $33,59(10)$ & $1,33(10)$ & 1 & BW5 & GSAS \\
Block 1 (vorgesintert) & 12,32 & $64,82(5)$ & $33,11(2)$ & $2,071(3)$ & $1^{*} 41$ & BW5 & MAUD \\
Scheibe (vorgesintert) & 13,32 & $64,5(6,5)$ & $33,4(3,4)$ & $2,18(22)$ & $1^{*} 41$ & BW5 & MAUD \\
Scheibe (vorgesintert) & 13,33 & $65,49(28)$ & $32,59(4)$ & $1,92(3)$ & 1 & BW5 & MAUD \\
Block 2 (vorgesintert) & 24,20 & $64,7(14,3)$ & $33,4(7,4)$ & $1,8(0,4)$ & $1^{*} 41$ & BW5 & MAUD \\
Mittelwert & & $\mathbf{6 4 , 9 2 ( 3 9 )}$ & $\mathbf{3 3 , 2 1 ( 3 9 )}$ & $\mathbf{1 , 8 7 ( 3 3 )}$ & & & \\
\hline Scheibe (La) & 18,33 & $67,92(12)$ & $30,58(4)$ & $1,50(1)$ & $1^{*} 41$ & BW5 & MAUD \\
Scheibe (La) & 9,17 & $67,19(7)$ & $27,79(3)$ & $5,02(1)$ & $1^{*} 39$ & W2 & MAUD \\
Mittelwert & & $\mathbf{6 7 , 6 ( 5 )}$ & $\mathbf{2 9 , 2 ( 2 , 0 )}$ & $\mathbf{3 , 3 ( 2 , 5 )}$ & & & \\
\hline \hline
\end{tabular}

${ }^{1}$ Zahl der aufgenommenen Beugungsbilder (1 = Einzelaufnahme; $1^{*} 41=1$ Positionshöhe mit 41 Beugungsbildern, die bei Drehung der Probe um $\omega$ in $4^{\circ}$-Schritten von $-80^{\circ}$ bis $+80^{\circ}$ aufgenommen wurden.) 
Tab. A.11: Ergebnisse aus den Rietveld-Verfeinerungen für Remanium GM 800+. Gemessen an der Synchrotron-Beamline P02.1 $\left(\lambda_{2}=0,2086 \AA\right)$ sowie am Bruker Smart Apex II $(\lambda=0,7093 \AA)$; ausgewertet mit den Programmen GSAS und MAUD.

\begin{tabular}{|c|c|c|c|c|c|c|c|c|}
\hline Beamline & Programm & $\begin{array}{l}\epsilon-\mathbf{C o C r}^{\mathbf{1}} \\
\left(P 6_{3} / m m c\right) \\
(\mathrm{Gew} . \%)\end{array}$ & $\begin{array}{c}\gamma- \\
\mathbf{C o C r}^{2} \\
(F m \overline{3} m) \\
(\text { Gew.\%) }\end{array}$ & $\begin{array}{c}\alpha- \\
\mathbf{M o C r}^{\mathbf{3}} \\
(\operatorname{Im} \overline{3} m) \\
\text { (Gew.\%) }\end{array}$ & $a_{\epsilon}(\AA)$ & $c_{\epsilon}(\AA)$ & $a_{\gamma}(\AA)$ & $a_{\alpha}(\AA)$ \\
\hline $\mathrm{P} 02.1^{4}$ & MAUD & $28,6(3)$ & $36,6(3)$ & $34,8(3)$ & $2,5363(2)$ & $4,0959(7)$ & $3,5843(3)$ & $2,8838(2)$ \\
\hline $\mathrm{P} 02.1^{4}$ & GSAS & $58,7(5)$ & $21,7(5)$ & $19,6(5)$ & $2,5260(38)$ & $4,0913(38)$ & $3,5807(52)$ & $2,8668(40)$ \\
\hline Bruker $^{5}$ & GSAS & $66,2(6)$ & $24,0(7)$ & $9,8(8)$ & $2,525(5)$ & $4,086(12)$ & $3,571(7)$ & $2,868(9)$ \\
\hline Mittelwert & $51(20)$ & $27(8)$ & $21(13)$ & $2,529(6)$ & $4,091(5)$ & $3,579(7)$ & $2,872(10)$ & \\
\hline
\end{tabular}

${ }^{1} \epsilon-\mathrm{CoCr}\left(P 6_{3} / m m c\right): \mathrm{Co}_{0,70} \mathrm{Cr}_{0,26} \mathrm{Mo}_{0,04}$

${ }^{2} \gamma-\mathrm{CoCr}(F m \overline{3} m): \mathrm{Co}_{0,68} \mathrm{Cr}_{0,24} \mathrm{Mo}_{0,08}$

${ }^{3} \alpha$-MoCr $(\operatorname{Im} \overline{3} m): \mathrm{Cr}_{0,85} \mathrm{Mo}_{0,15}$

${ }^{4}$ Gleiche Messung (P02.1; $\lambda=0,2086 \AA$; Probe-Detektor-Abstand: $\left.1000 \mathrm{~mm}\right)$, Auswertung mit zwei verschiedenen Programmen $\left(R_{w p}(\right.$ MAUD $)=5,9 \%, R_{w p}($ GSAS $\left.)=16,33 \%\right)$

${ }^{5}$ Messung am Bruker $\left(\lambda=0,7093 \AA\right.$; Probe-Detektor-Abstand: $\left.600 \mathrm{~mm} ; R_{w p}=9,74 \%\right)$

Tab. A.12: Übersicht über verfeinerte d-Werte für die verschiedenen Glasphasen. Geschätzte Standardabweichungen aus der Rietveld-Analyse sind in Klammern angegeben.

\begin{tabular}{l|cc}
\hline \hline Probe & $d_{\text {Glas }}(\AA)$ & $\omega$ \\
\hline Vita In-Ceram Alumina & $1,571(13)$ & $-60^{\circ}$ bis $60^{\circ}$ in $30^{\circ}$-Schritten \\
Vita In-Ceram Zirkonia & $1,536(4)$ & $-60^{\circ}$ bis $60^{\circ}$ in $30^{\circ}$-Schritten \\
IPS e.max Press, Rohling & $1,722(9)$ & $-60^{\circ}$ bis $60^{\circ}$ in $30^{\circ}$-Schritten \\
IPS e.max Press, Gusskanal & $1,780(10)$ & $-60^{\circ}$ bis $60^{\circ}$ in $30^{\circ}$-Schritten \\
Vitablocs Esthetic Line & $1,83(8)$ & $-80^{\circ}$ bis $80^{\circ}$ in $10^{\circ}$-Schritten \\
Standardmischungen $(\alpha$-Carnegieit $)$ & $2,042(2)$ & $-60^{\circ}$ bis $60^{\circ}$ in $30^{\circ}$-Schritten \\
\hline \hline
\end{tabular}

Tab. A.13: Einkristalldaten für die thermische Ausdehnung von $\mathrm{Li}_{2} \mathrm{Si}_{2} \mathrm{O}_{5}$, Sanidin, Korund und Titan. Nicht angegebene Terme nehmen den Wert 0 an. Alle Angaben sind in $10^{-6} / \mathrm{K}$.

\begin{tabular}{l|ccc|l}
\hline \hline Phase & $a_{11}$ & $a_{22}$ & $a_{33}$ & Quelle \\
\hline $\mathrm{Li}_{2} \mathrm{Si}_{2} \mathrm{O}_{5}$ & 8,3 & 17,0 & 6,8 & Mastelaro \& Zanotto (1999) \\
Sanidin & 1,3 & 19,8 & 1,6 & Henderson (1979) \\
Korund & 5,2 & 5,2 & 5,9 & Shvyd'ko et al. (2002) \\
Titan & 9,5 & 9,5 & 5,6 & Pawar \& Deshpande (1968) \\
\hline \hline
\end{tabular}




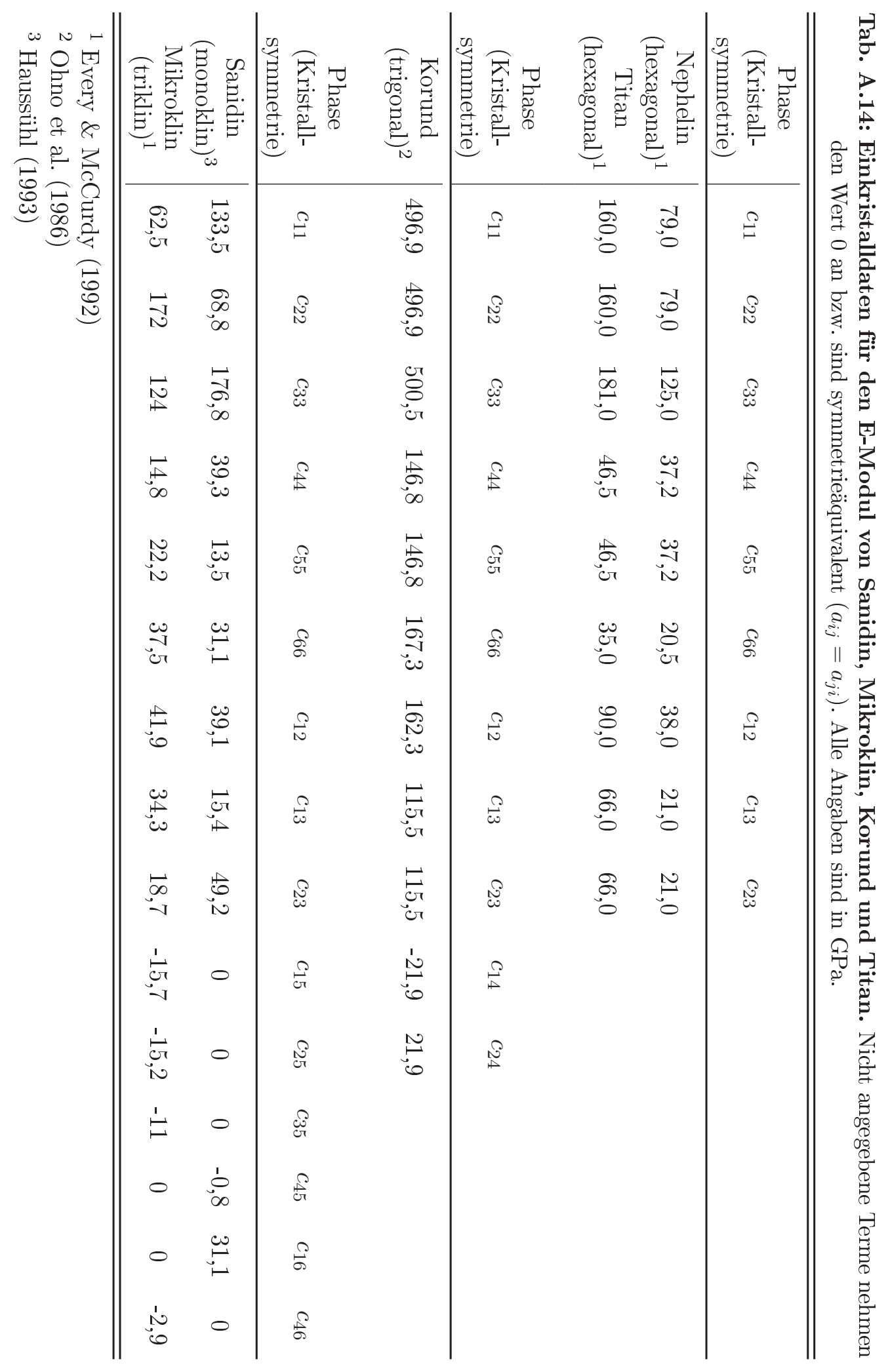




\section{B Abbildungen}

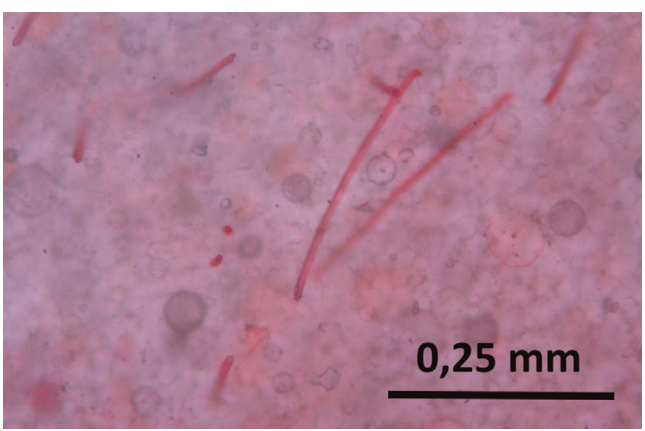

(a) Übersichtsaufnahme: Zu sehen sind rote, abtauchende und parallel liegende Kunststofffasern in einer hellroten PMMA-Matrix.

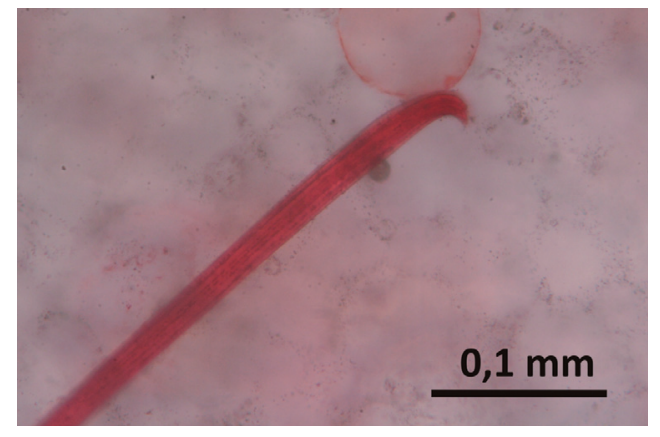

(b) Eine Kunststofffaser parallel zur Schnittfläche.

Abb. B.1: Transmissionsbilder einer ca. $3 \mathrm{~mm}$ dicken Scheibe des Prothesenkunststoffes Aesthetic mit normalem Faseranteil.

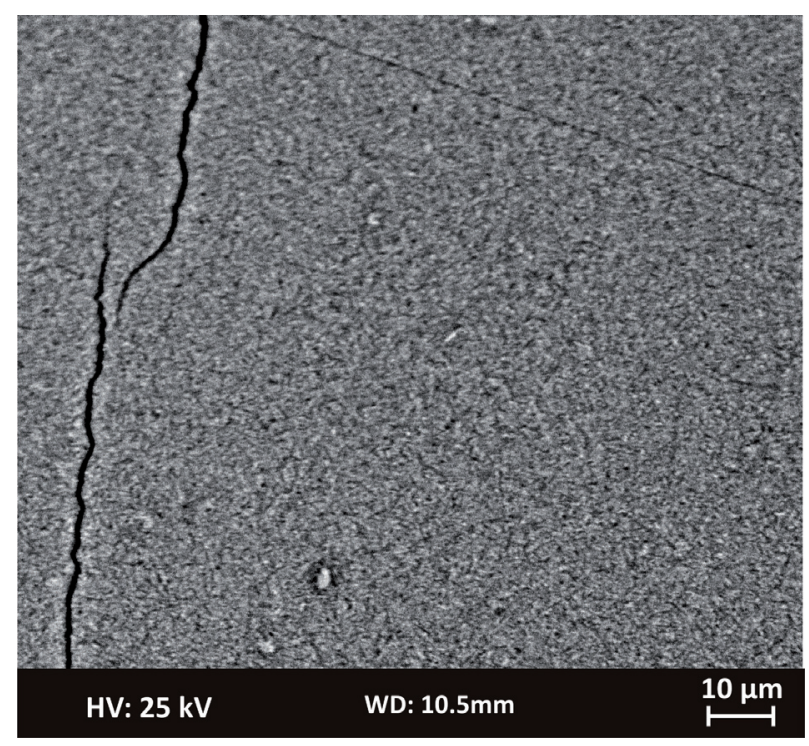

Abb. B.2: Rückstreuelektronenbild der Lithiumdisilikat-Keramik IPS e.max Press im vorgesinterten Zustand. Die Keramik erscheint sehr feinkörnig und ohne starke Massenkontraste. Risse rühren von der Präparation des Dünnschliffes her. 


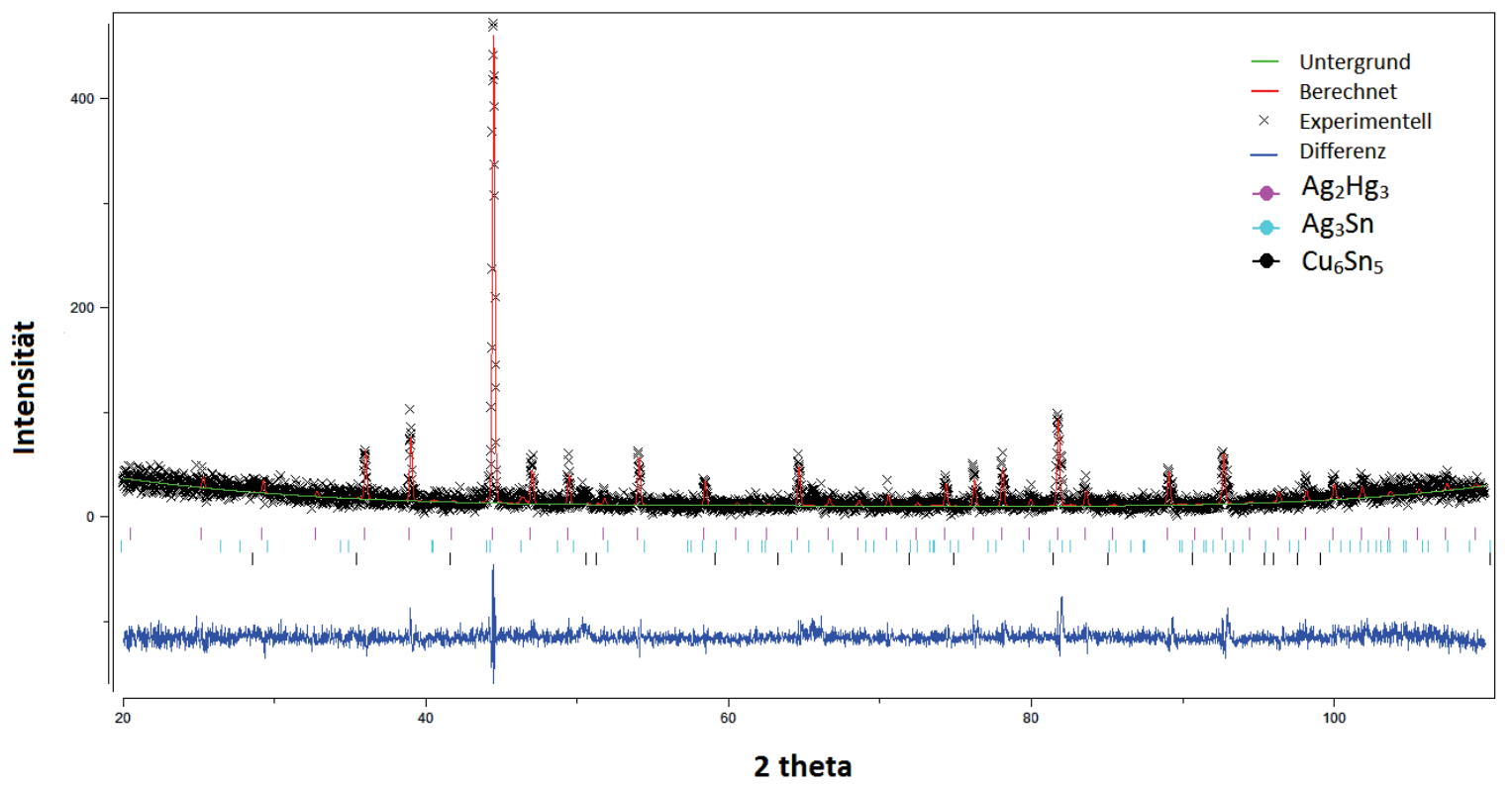

(a) Röntgendiffraktogram aufgenommen am XRD $3000 \operatorname{PTS}(\lambda=1,79 \AA)$.

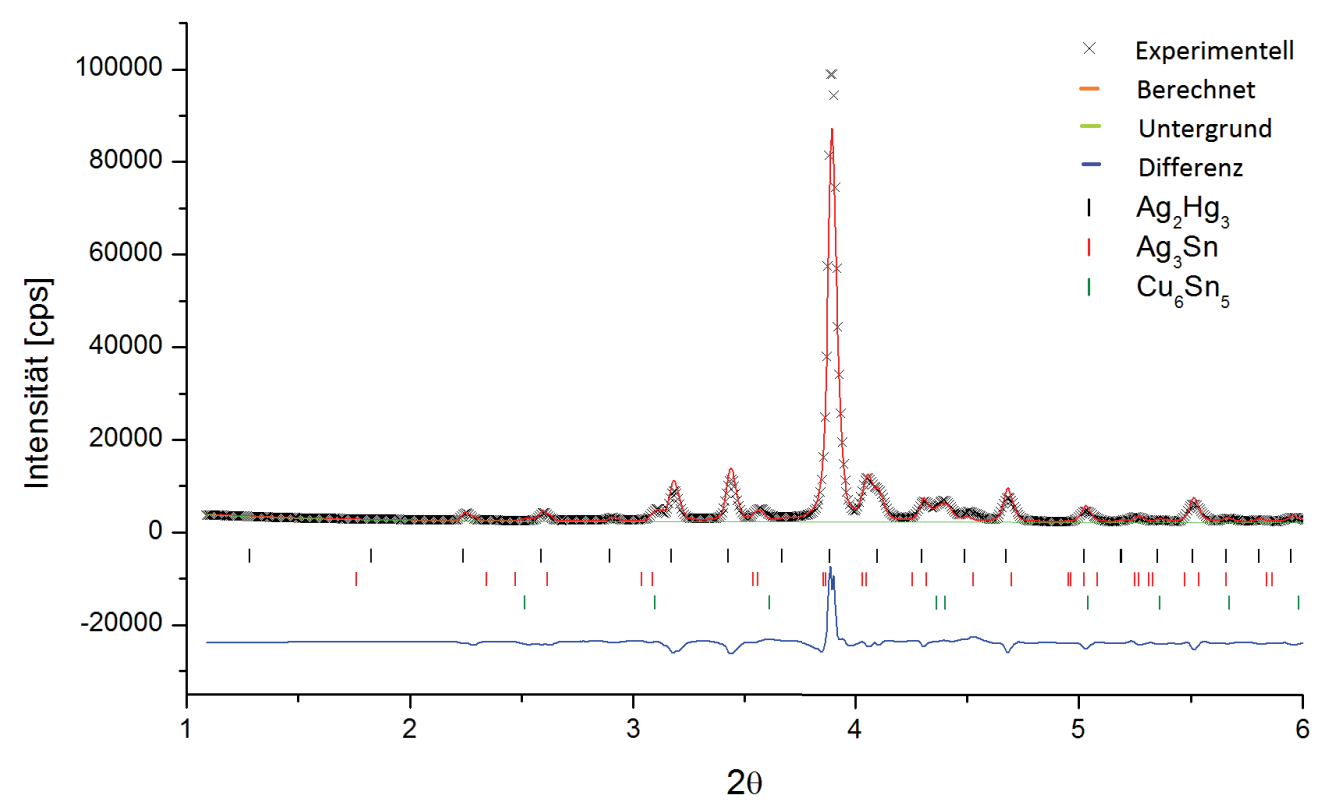

(b) Röntgendiffraktogram aufgenommen am BW5 $(\lambda=0,1613 \AA)$.

Abb. B.3: Röntgendiffraktogramme des Dentalamalgams Amalcap regular. Gemessen an dem Röntgendiffraktometer XRD 3000 PTS (a) und an der Beamline BW5 (b). Ausgewertet mit dem Programm GSAS. 


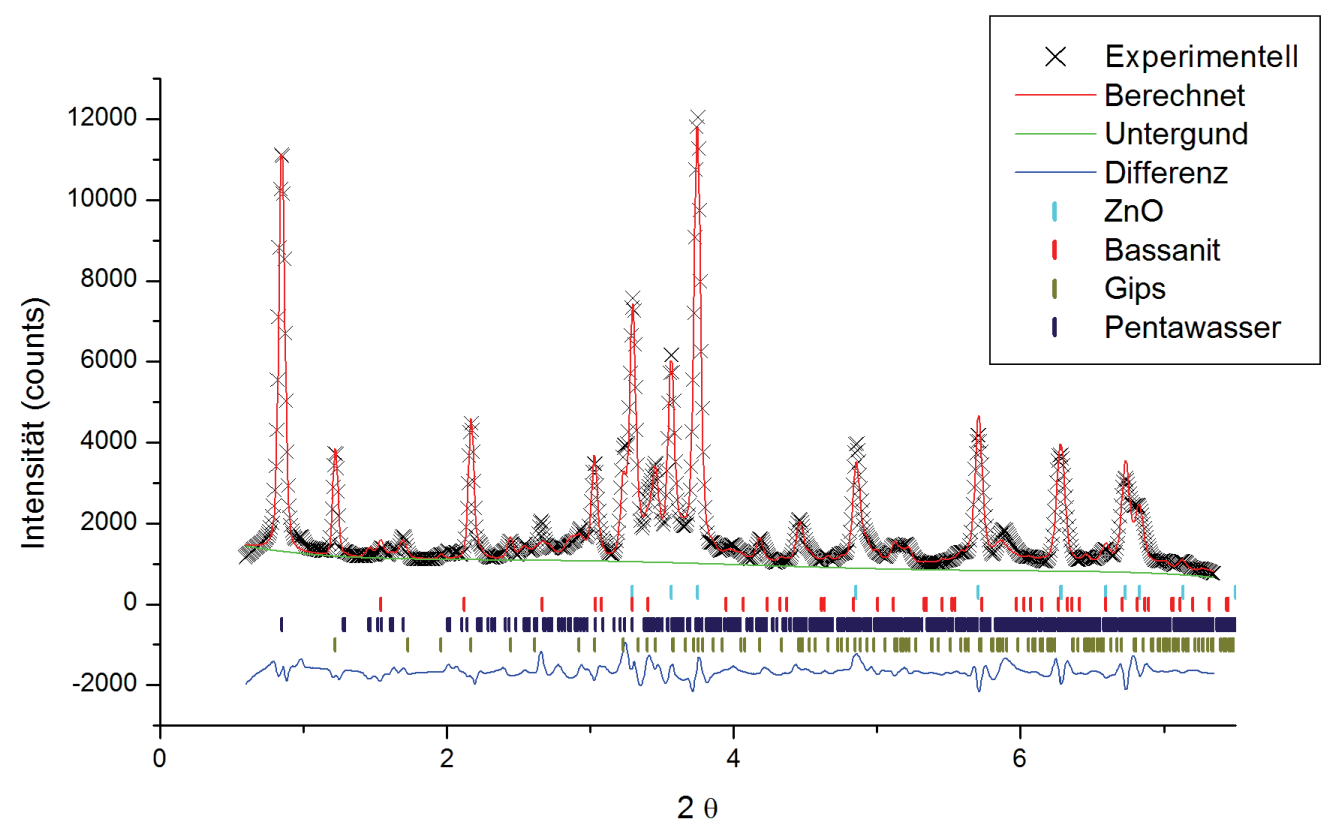

(a) Detaferm

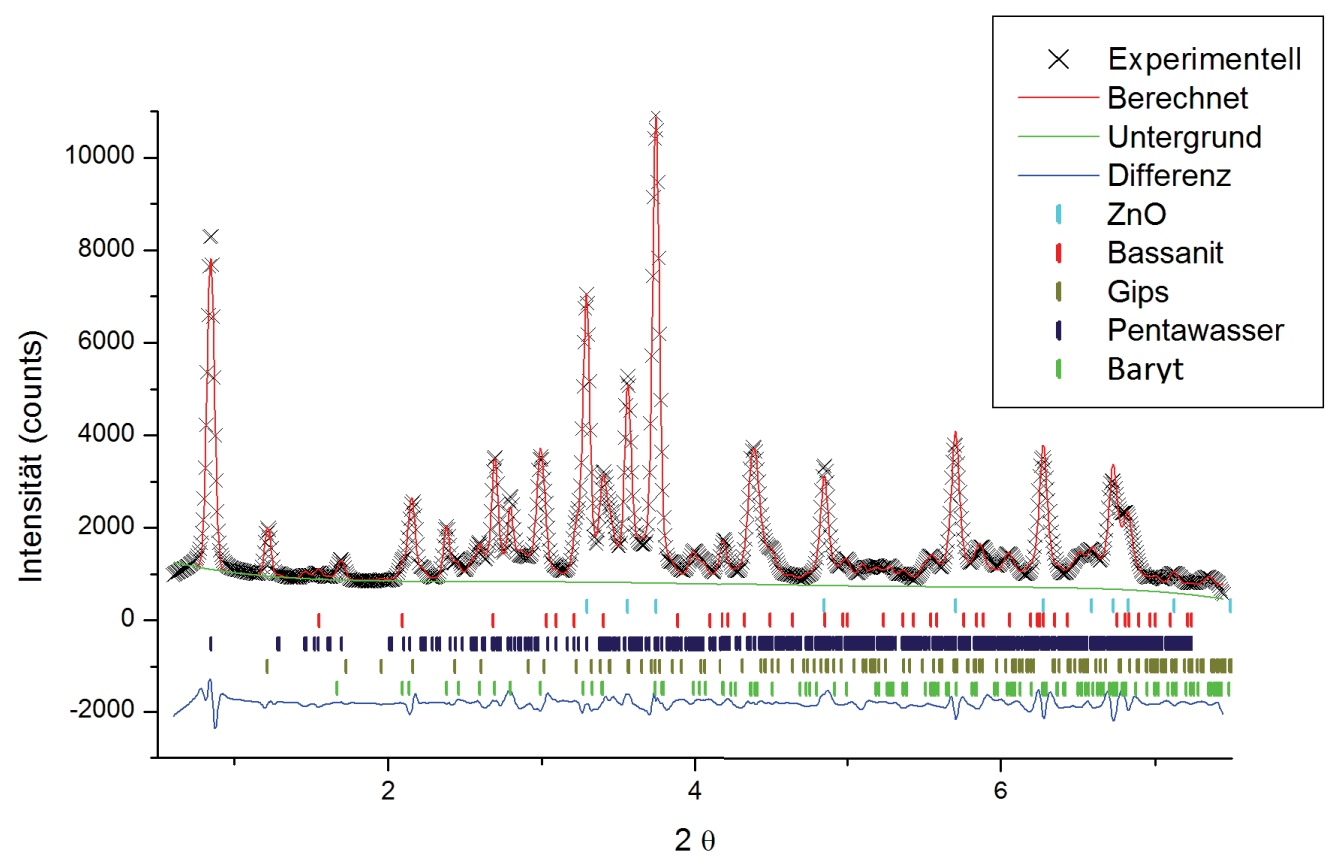

(b) Cavit

Abb. B.4: Röntgendiffraktogramme der Zinksulfatzemente nach einer Reaktionszeit von 3 Tagen. Aufgenommen an der Beamline BW5 $(\lambda=0,1613 \AA)$. Ausgewertet mit dem Programm GSAS. 


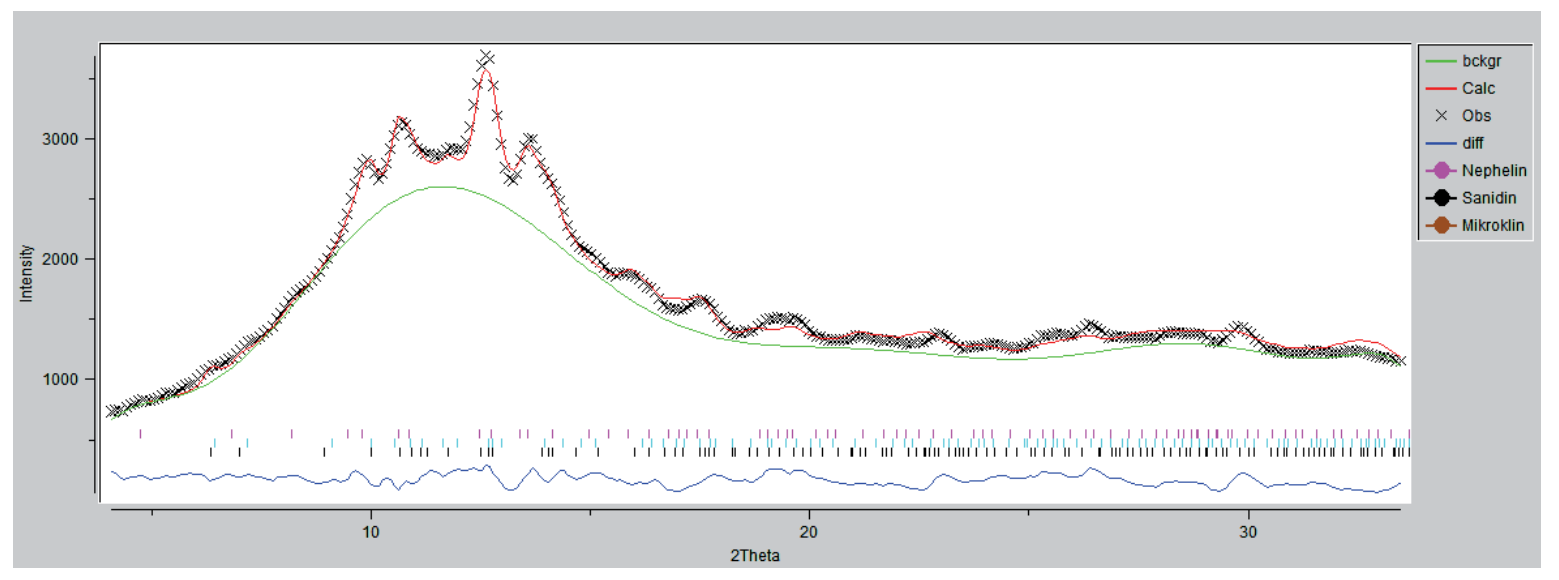

Abb. B.5: Röntgendiffraktogramm der Dentalkeramik Vitablocs - Esthetic Line (Bruker $S M A R T$ APEX II, $\left.\lambda_{K \bar{\alpha}}=0,7093 \AA\right)$.
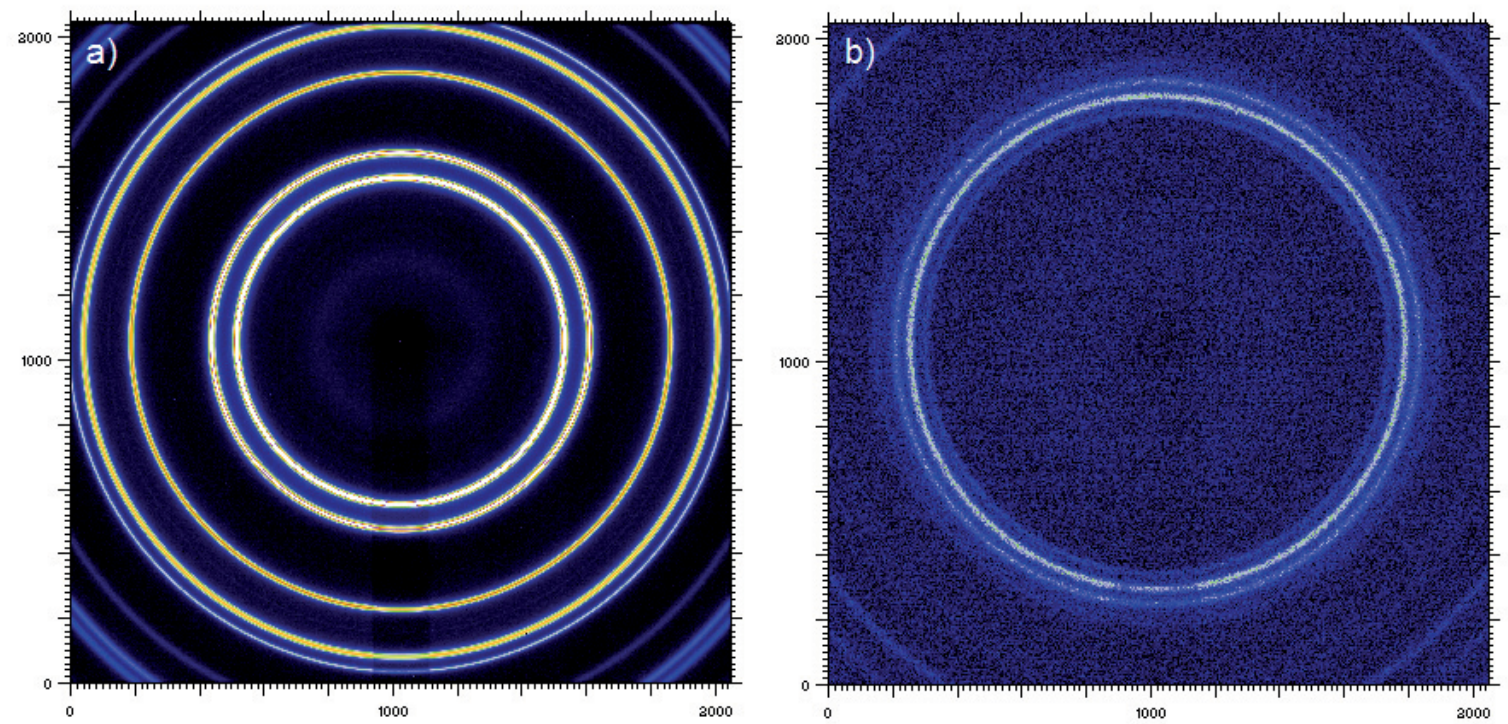

Abb. B.6: Transmissionsbilder der pulverisierten und in Glaskapillaren gefüllten Legierungen Wirong9 (a, Ni-Cr-Mo-Legierung) und Remanium GM 800+ (b, Co-Cr-MoLegierung). Messplatz P02.1, $\lambda=0,20868 \AA$. 


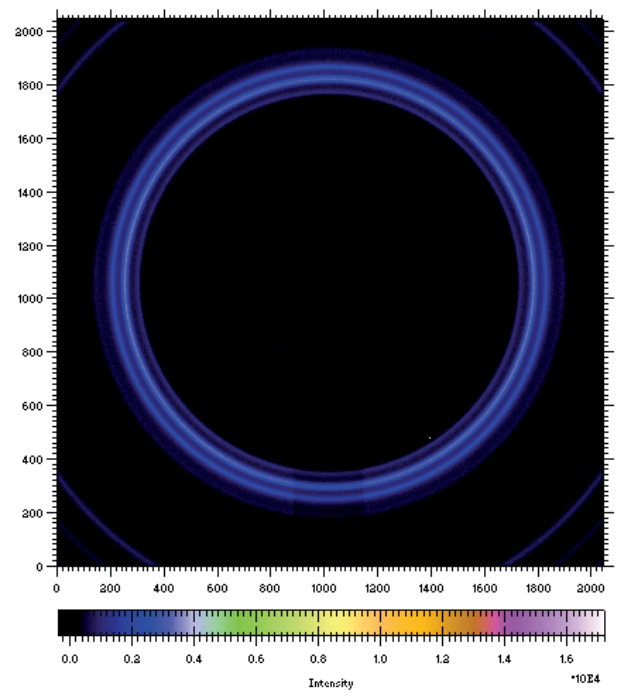

(a) Transmissionsbild.

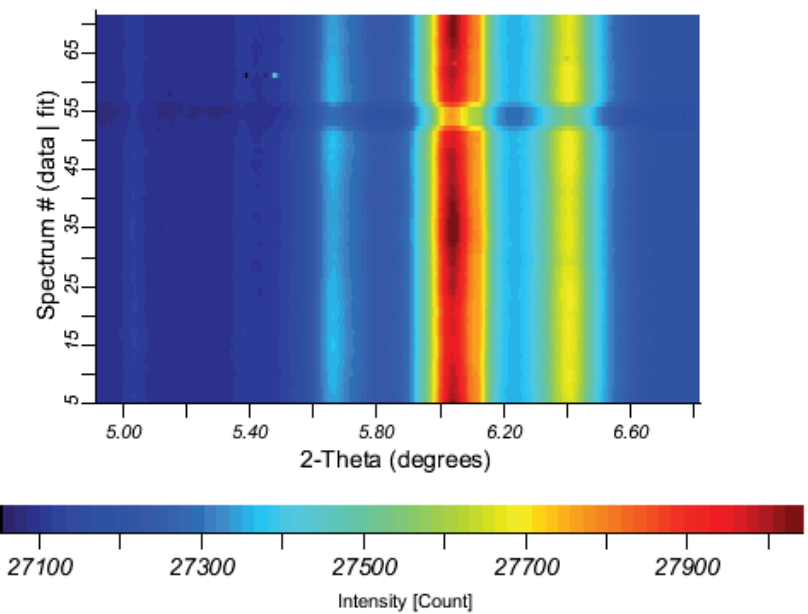

(b) 2d-Multiplot erstellt mit MAUD.

Abb. B.7: Transmissionsbild und 2d-Multiplot der Co-Cr-Mo-Legierung Remanium GM $\mathbf{8 0 0}+$ (Pulver in Glaskapillare). In (a) erscheinen die Beugungsringe fast frei von Textureinflüssen, während in (b) deutliche Intensitätsmaxima auf dem stärksten Reflex zu sehen sind. Messplatz P02.1, $\lambda=0,20868 \AA$.

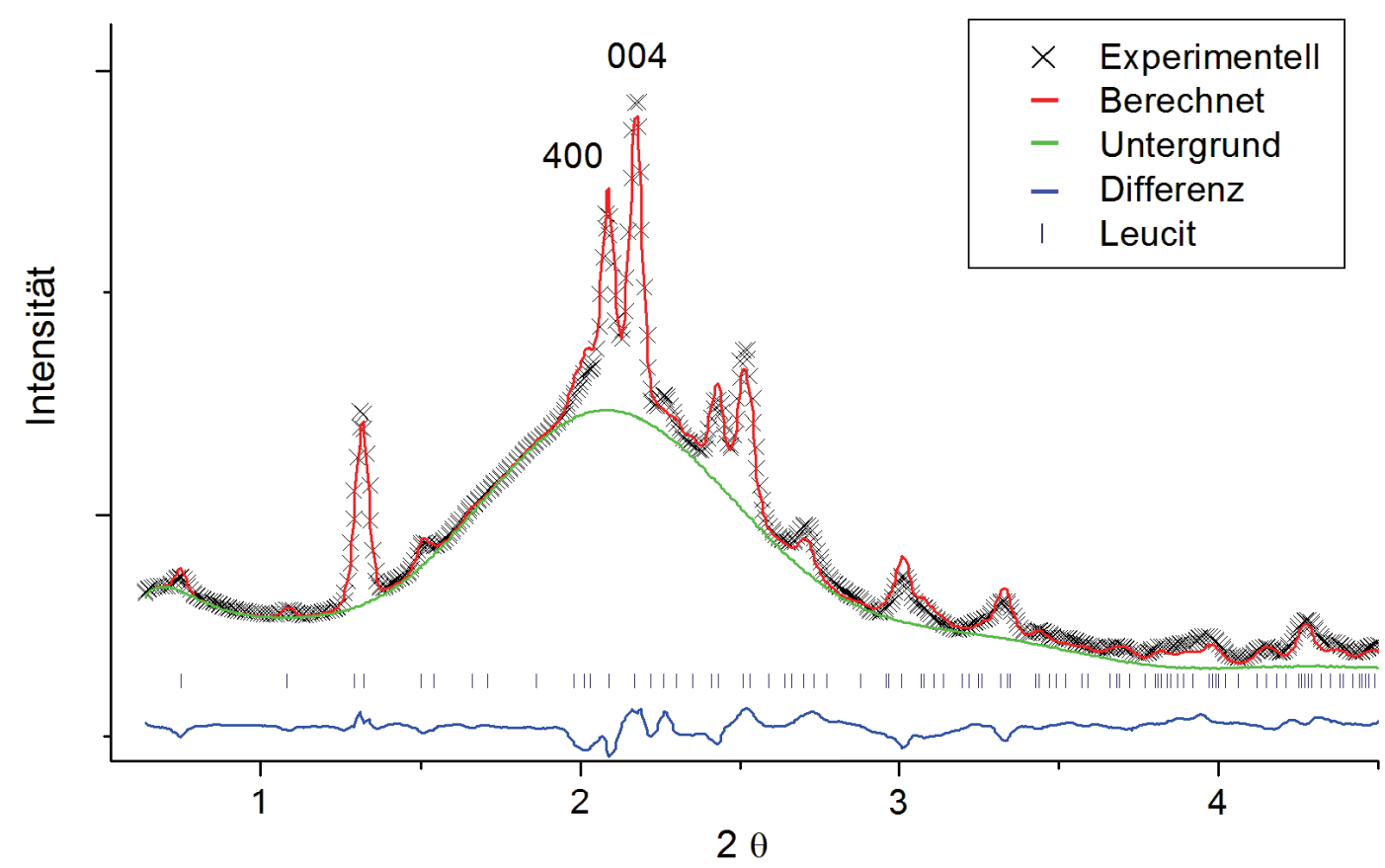

Abb. B.8: Röntgendiffraktogramm der Verblendkeramik Duceram Kiss für die Goldlegierung Degudent $\boldsymbol{U}$. Ausgewertet mit dem Programm GSAS. Messplatz BW5, $\lambda=$ $0,1248 \AA$. 


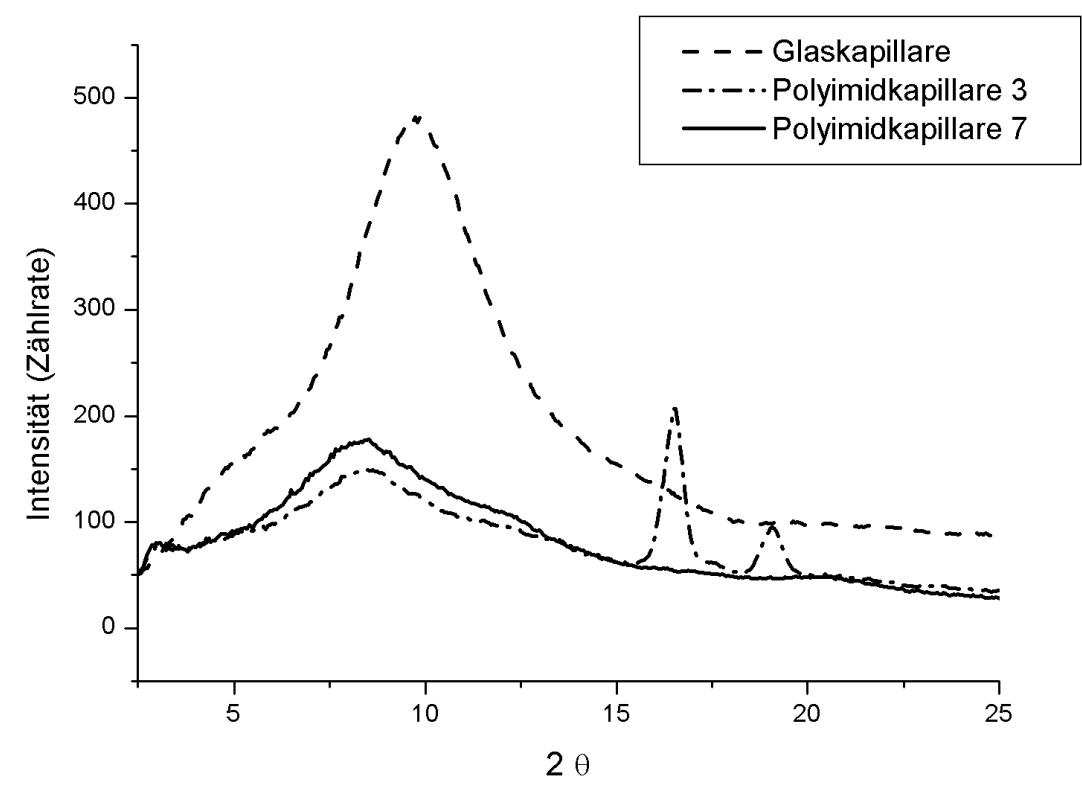

Abb. B.9: Röntgendiffraktogramme einer Glaskapillare $\emptyset^{\text {außen }}=0,8 \mathrm{~mm}$ ), einer PIKapillare mit kristallinem Gehalt (Nr. 3) und ohne kristallinem Gehalt (Nr. 7) im Vergleich. Messung am Bruker SMART APEX II ( $\lambda=\mathrm{Mo}_{\alpha} \mathrm{K}_{\alpha}(0,7107 \AA), 50 \mathrm{kV}$, $25 \mathrm{~mA}, 60$ s Belichtungszeit). Abb. nach Raue (2013). 


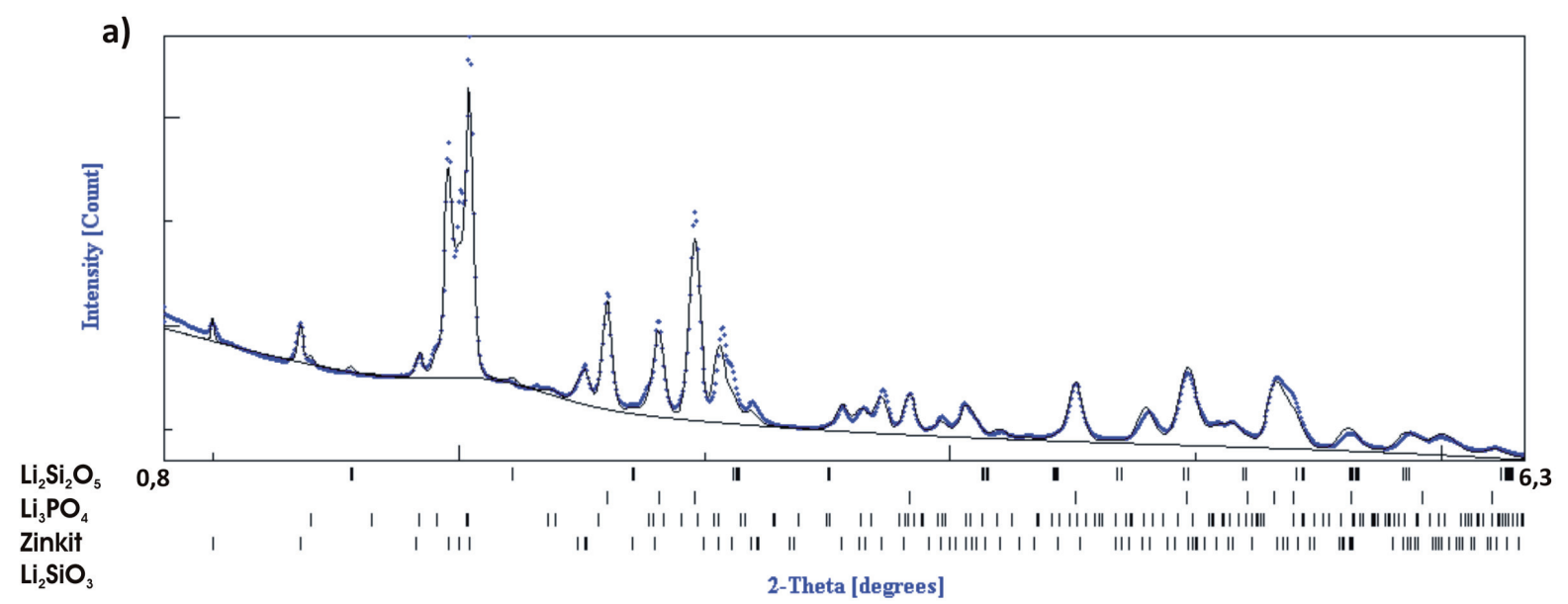

b)

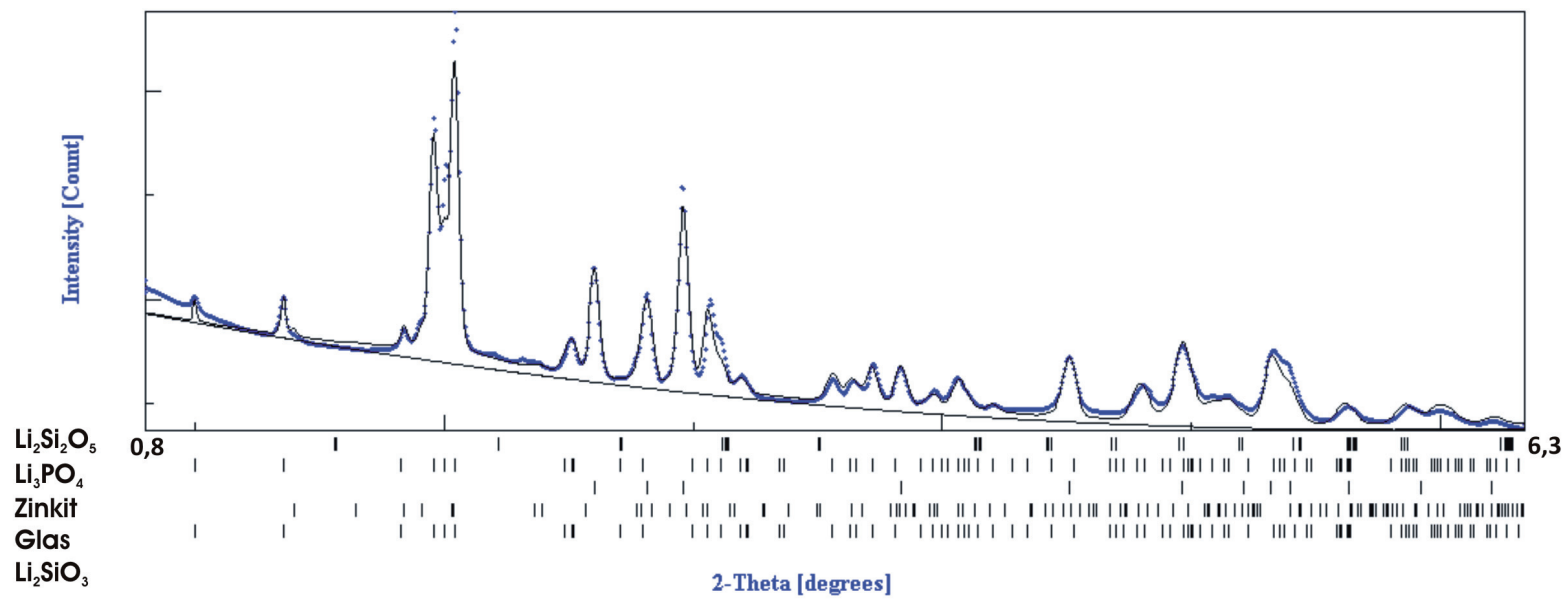

Abb. B.10: Röntgendiffraktogramme der LS2-Keramik IPS e.max Press in PI-Kapillare. (a) zeigt Profilanpassung nach der IS-Methode (Streuung des Glases durch breiten GaussPeak angepasst) und (b) nach der GS-Methode $\left(\mathrm{Li}_{2} \mathrm{Si}_{2} \mathrm{O}_{5}\right.$ mit $14 \AA$ als Kristallitgröße zur Simulation der Glasphase). Messung am BW5 $(\lambda=0,127845 \AA)$. Ausgewertet mit dem Programm MAUD. 


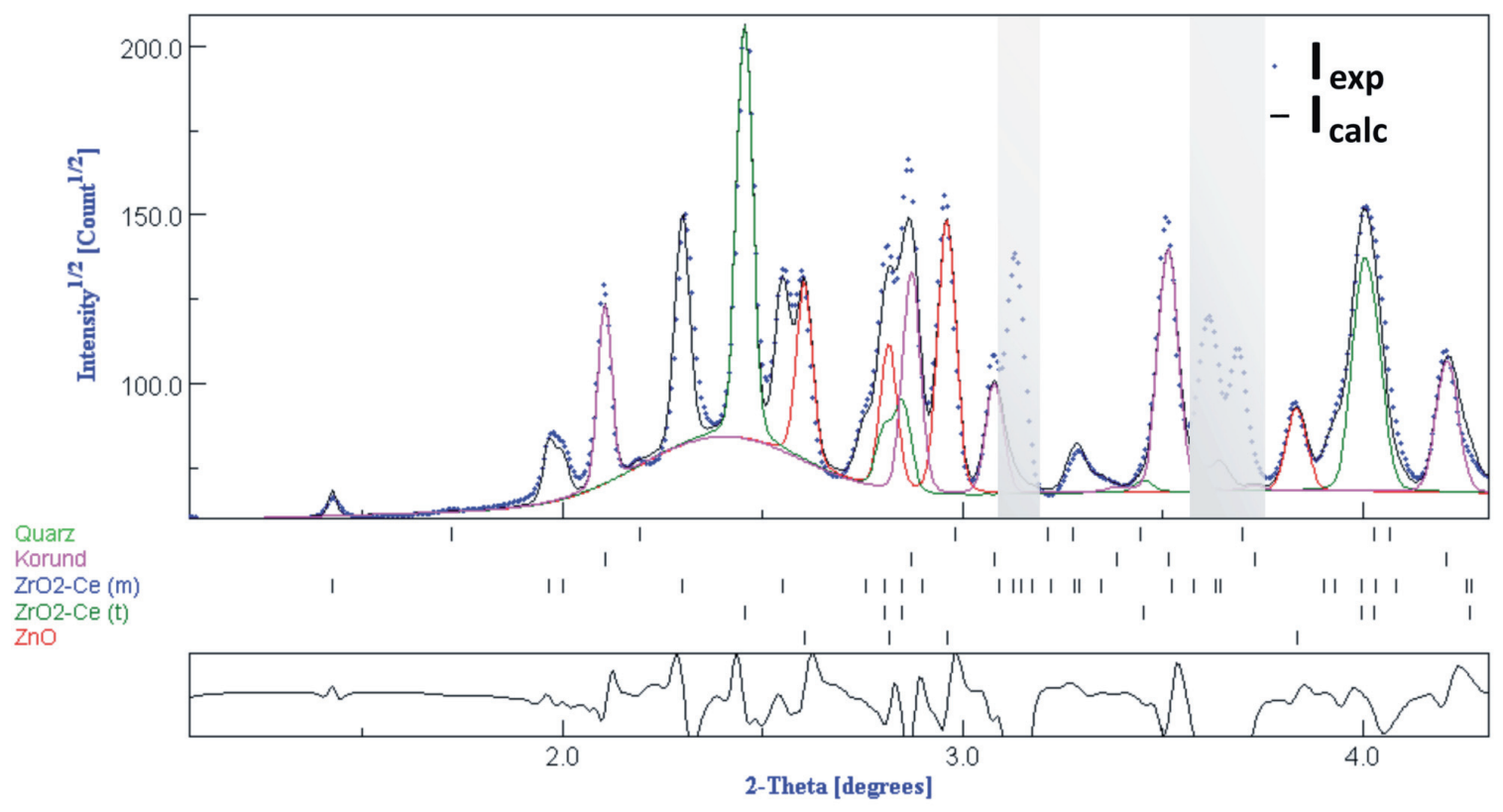

(a) Rietveld-Verfeinerung nach der IS-Methode. Der Untergrund wurde durch 4 Parameter und einen Gausspeak bei $2,401(1)^{\circ} 2 \theta$ (Halbwertsbreite $=0,224(1)$ ) angepasst. Die berechneten Reflexe aller Phasen zusammen sind in schwarz dargestellt. Zusätzlich sind die Reflexe der einzelnen Phasen Korund, tetragonales $\mathrm{ZrO}_{2}$-Ce (t) und Zinkit farbig eingetragen.

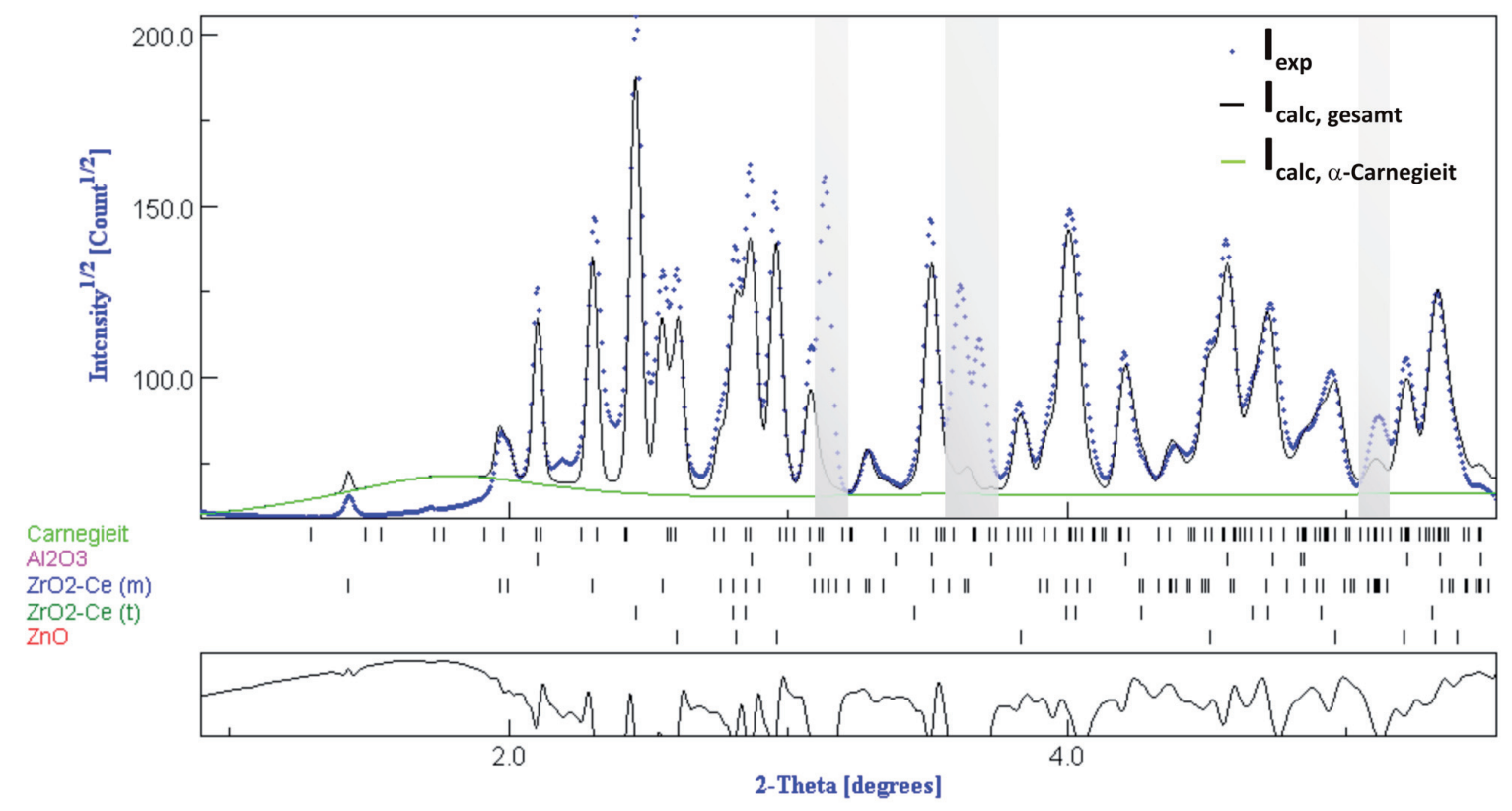

(b) Gleiche Rietveld-Verfeinerung wie in (a), wobei die Streunung des amorphen Glasuntergrunds nicht durch einen Gausspeak simuliert wurde (linearer Untergrund, 2 Parameter). Stattdessen wurden 20 Gew.\% $\alpha$-Carnegieit als Glasphase eingeführt, dessen Streuungsmaximum bei $2 \theta=1,80^{\circ}$ liegt. Bei Verfeinerung geht der Gewichtsanteil von $\alpha$-Carnegieit gegen Null.

Abb. B.11: Röntgendiffraktogramme der Keramik Vita In-Ceram Zirkonia (Pulver mit Zusatz von $\mathrm{ZnO}$ in Alu-Röhrchen). Grau hinterlegte Bereiche markieren Aluminium-Reflexe des Behälters (Al-Röhrchen) und wurden in den Rietveld-Verfeinerungen nicht berücksichtigt (BW5; $\lambda=0,12785 \AA)$. 

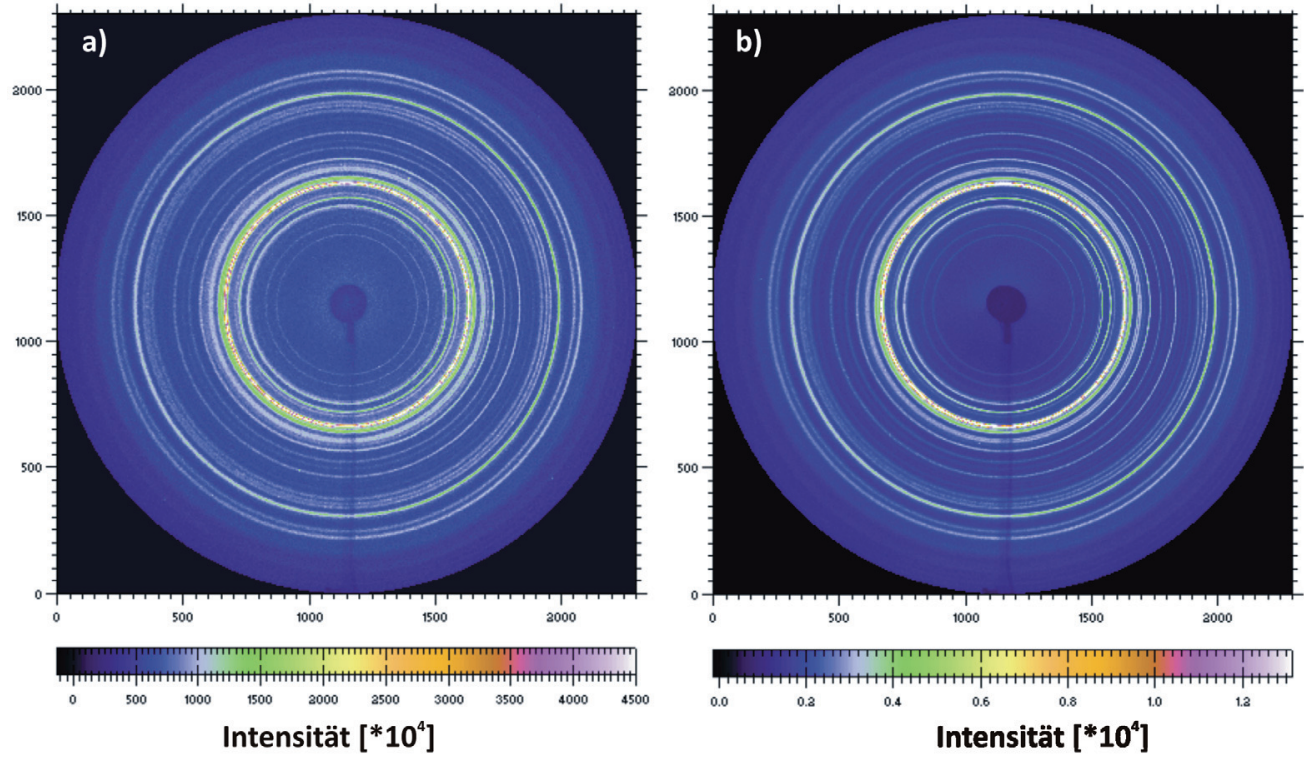

Abb. B.12: 2d-Transmissionsaufnahmen der Dentalamalgame Amalcap regular (a) und Amalcap fast (b). Messplatz BW5, $\lambda=0,1248 \AA$.

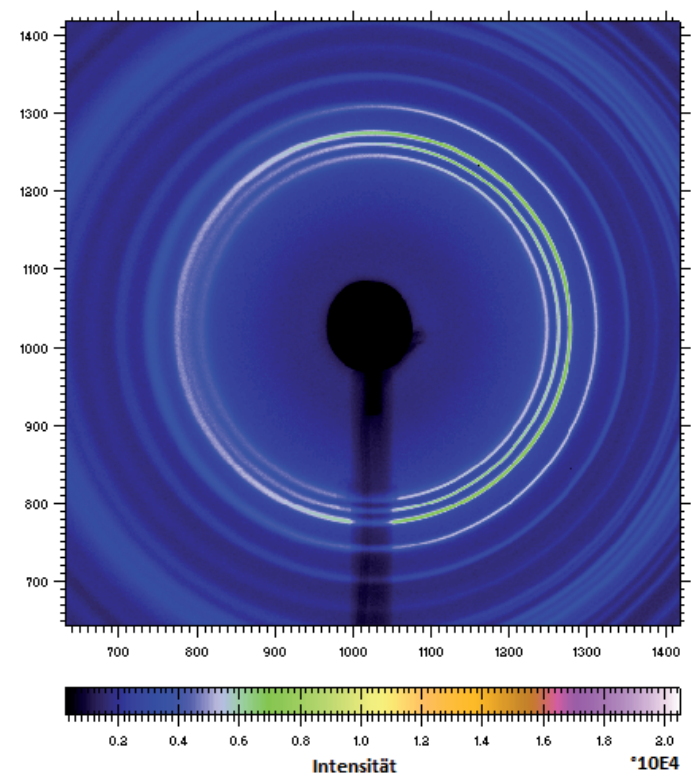

Abb. B.13: 2d-Transmissionsaufnahme des Dentalkomposits Tetric Ceram. Beugungsringe stammen von $\mathrm{YbF}_{3}-$ Kristalliten und weisen keine Beugungsmaxima, sondern nur eine Rechts/Link-Asymmetrie bedingt durch den Messaufbau auf (BW5, $\lambda=0,1241 \AA$ ). 


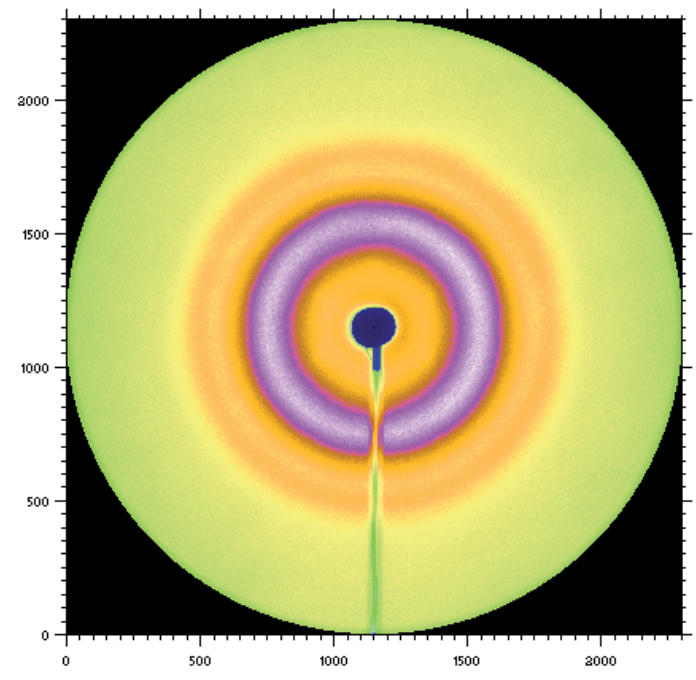

Abb. B.14: 2d-Transmissionsaufnahme des Dentalkomposits Rebilda LC. Aufgenommen am BW5, $\lambda=0,1613 \AA$.

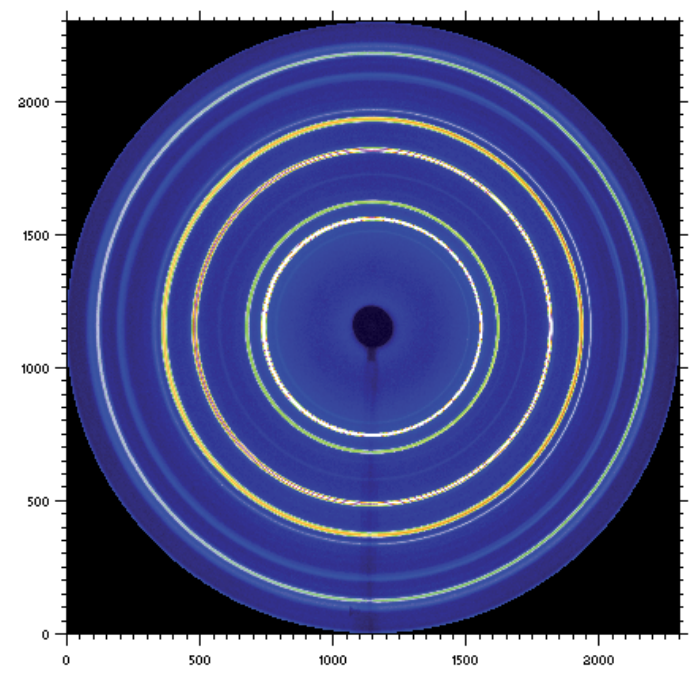

Abb. B.15: 2d-Transmissionsaufnahme der $\mathrm{Y}^{-\mathrm{ZrO}_{2}}$-Keramik Cercon base. Beugungsringe stammen von der tetragonal stabilisierten $\mathrm{Y}^{-} \mathrm{ZrO}_{2}$-Phase und weisen keine Beugungsmaxima auf (BW5, $\lambda=0,1255 \AA)$. 


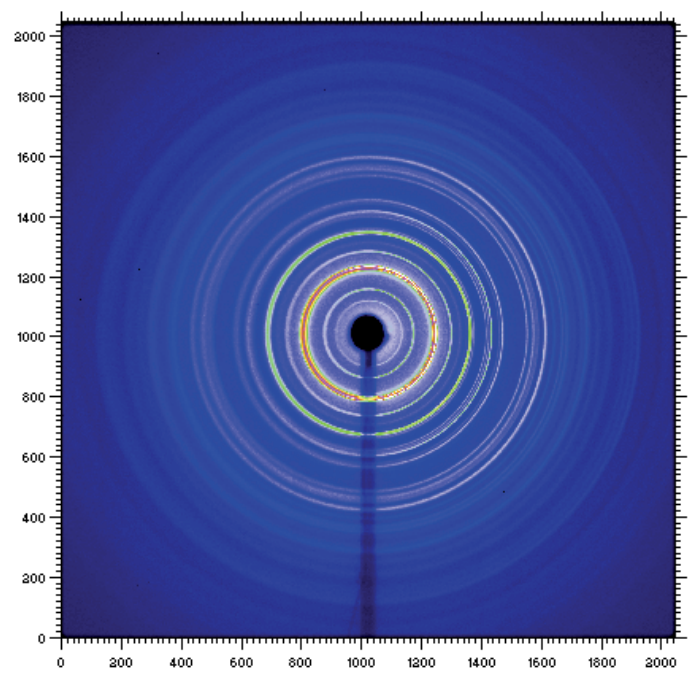

Abb. B.16: 2d-Transmissionsaufnahme der $\mathrm{Li}_{2} \mathrm{Si}_{2} \mathrm{O}_{5}$-Keramik IPS e.max CAD. Beugungsringe stammen von den kristallinen Phasen $\mathrm{Li}_{2} \mathrm{Si}_{2} \mathrm{O}_{5}, \mathrm{Li}_{3} \mathrm{PO}_{4}$ und $\mathrm{Li}_{2} \mathrm{SiO}_{3}$ und weisen keine Beugungsmaxima auf (BW5, $\lambda=0,1239 \AA$ ).

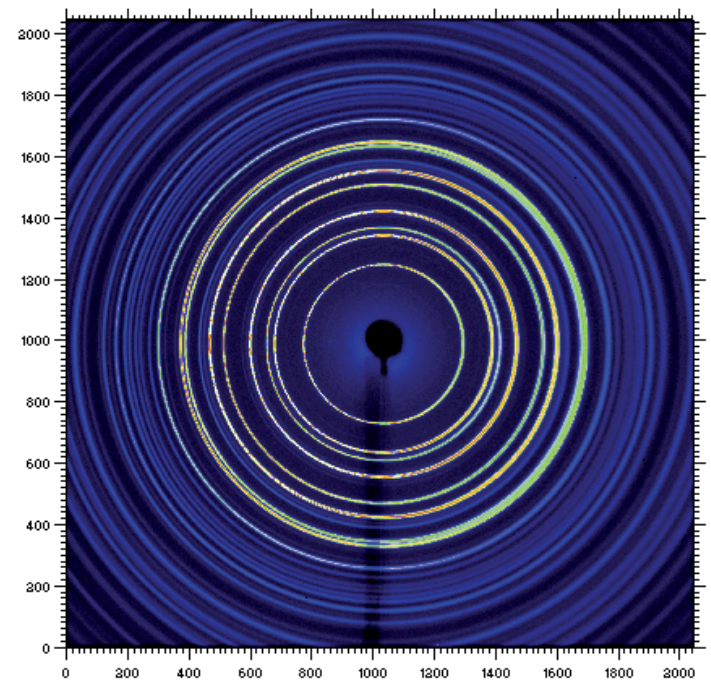

Abb. B.17: 2d-Transmissionsaufnahme der Korund-Keramik Vita In-Ceram Alumina. Beugungsringe stammen von der Korundphase und weisen keine Beugungsmaxima auf (BW5, $\lambda=0,1280 \AA)$. 